NUREG/CR-6157

LA-12715-MS

\title{
Survey and Evaluation of Aging Risk Assessment Methods and Applications
}

Manuscript Completed: July 1993

Date Published: November 1994

Prepared by

D. Sanzo, P. Kvam/LANL

G. Apostolakis, J. Wu, T. Milici, N. Ghoniem, S. Guarro/ASCA

Los Alamos National Laboratory

Los Alamos, NM 87545

Advanced System Concepts Associates

2250 E. Imperial Highway

El Segundo, CA 90245-3547

\section{Prepared for}

Division of Safety Issue Resolution

Office of Nuclear Regulatory Research

U.S. Nuclear Regulatory Commission

Washington, DC 20555-0001

NRC FIN L2178

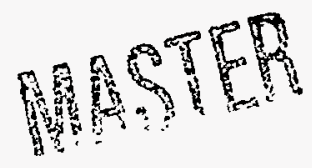

DISTRIOUTHON OF THIS COEUARENT RS UNLINATED 
. 


\section{DISCLAIMER}

This report was prepared as an account of work sponsored by an agency of the United States Government. Neither the United States Government nor any agency thereof, nor any of their employees, make any warranty, express or implied, or assumes any legal liability or responsibility for the accuracy, completeness, or usefulness of any information, apparatus, product, or process disclosed, or represents that its use would not infringe privately owned rights. Reference herein to any specific commercial product, process, or service by trade name, trademark, manufacturer, or otherwise does not necessarily constitute or imply its endorsement, recommendation, or favoring by the United States Government or any agency thereof. The views and opinions of authors expressed herein do not necessarily state or reflect those of the United States Government or any agency thereof. 


\section{DISCLAIMER}

Portions of this document may be illegible in electronic image products. Images are produced from the best available original document. 


\begin{abstract}
The US Nuclear Regulatory Commission initiated the nuclear power plant aging research program about 6 years ago to gather information about nuclear power plant aging. Since then, this program has collected a significant amount of information, largely qualitative, on plant aging and its potential effects on plant safety. However, this body of knowledge has not yet been integrated into formalisms that can be used effectively and systematically to assess plant risk resulting from aging, although models for assessing the effect of increasing failure rates on core damage frequency have been proposed.

This report surveys the work on the aging of systems, structures, and components (SSCs) of nuclear power plants, as well as associated data bases. We take a critical look at the need to revise probabilistic risk assessments (PRAs) so that they will include the contribution to risk from plant aging, the adequacy of existing methods for evaluating this contribution, and the adequacy of the data that have been used in these evaluation methods. We identify a preliminary framework for integrating the aging of SSCs into the PRA and include the identification of necessary data for such an integration.
\end{abstract}


- 


\section{CONTENTS}

Page

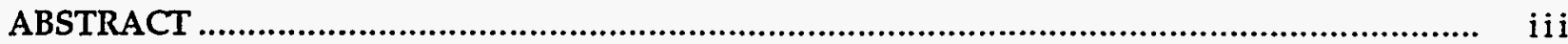

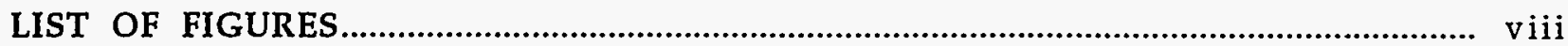

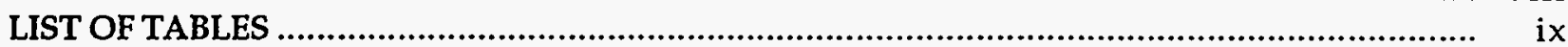

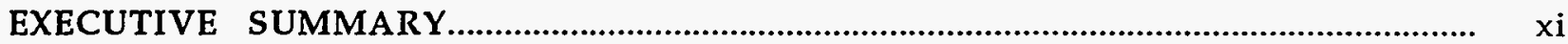

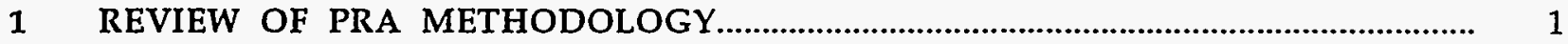

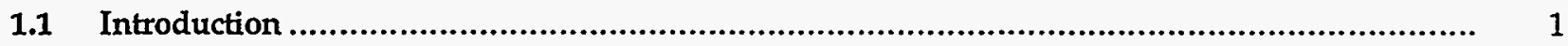

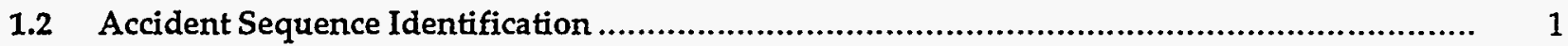

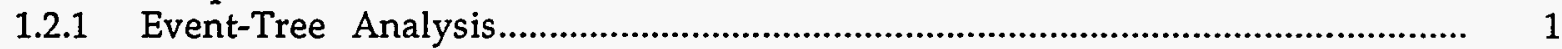

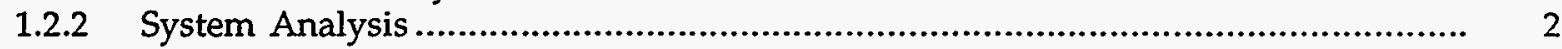

1.2.2.1 Scope of the System Analysis ................................................................... 3

1.2.2.2 Qualitative System Analysis............................................................. 4

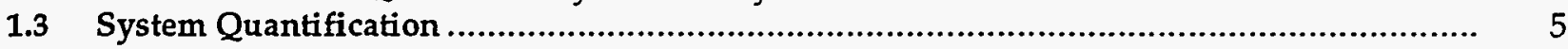

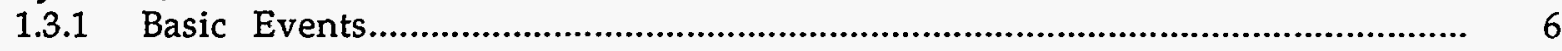

1.3.1.1 Component Failure Distribution............................................................. 6

1.3.1.2 Common-Cause Failures .......................................................................... 7

1.3.1.3 Pre-Accident Human Errors.................................................................. 10

1.3.1.4 Component Unavailability Because of Test and Maintenance.................... 10

1.3.2 The Quantification of System Unavailability .................................................. 11

1.3.2.1. System Unavailability Under Periodic Test and Maintenance................ 11

1.3.2.2. Correlation Among Statistical Parameters............................................... 13

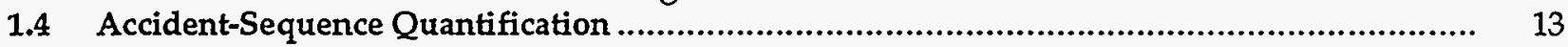

1.4.1 Logical Approaches for Accident-Sequence Quantification .................................... 13

1.4.2 Post-Accident Human Error.................................................................................... 14

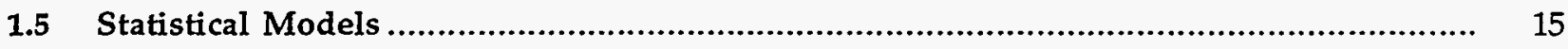

1.5.1 Subjective vs Relative-Frequency Interpretation of Probability ............................ 16

1.5.1.1 Relative-Frequency Interpretation of Probability ................................... 16

1.5.1.2 Subjective Theory of Probability............................................................. 16

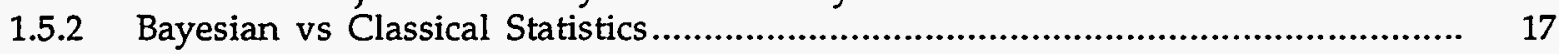

1.5.2.1 Classical Statistics......................................................................... 17

1.5.2.2 Bayesian Statistics ................................................................ 17

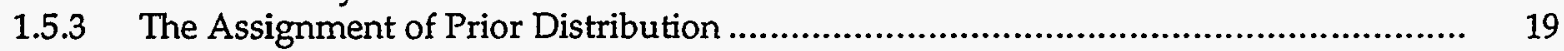

1.5.3.1 Reactor Safety Study Approach ....................................................... 19

1.5.3.2 The Use of IEEE Std-500 Data................................................................. 19

1.5.3.3 Two-Stage Bayesian ....................................................................... 20

1.6 External Events Analysis-Seismic Risk Assessment ................................................... 20

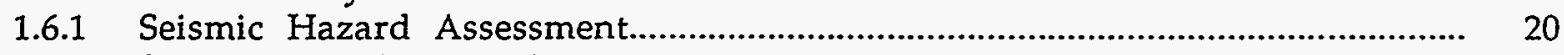

1.6.2 Seismic Fragility Analysis......................................................................... 21

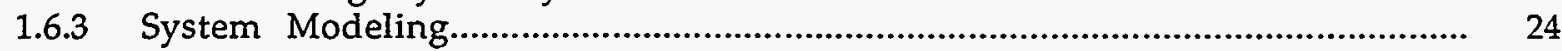

2 AGING RISK ANALYSES AND PHYSICAL MODELS ........................................... 25

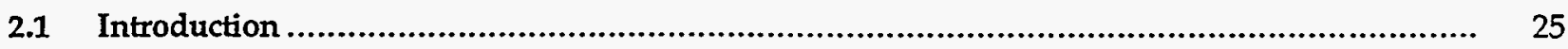

2.2 Taylor Expansion Approach for Incorporating Aging Effects into PRAs........................... 25

2.2.1 The Model's Objectives................................................................................. 25

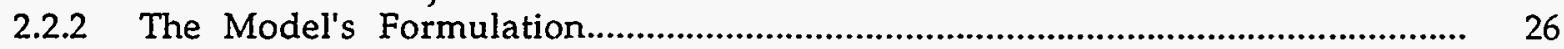

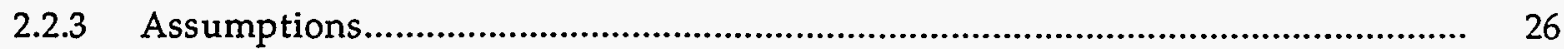

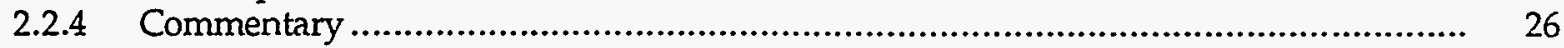

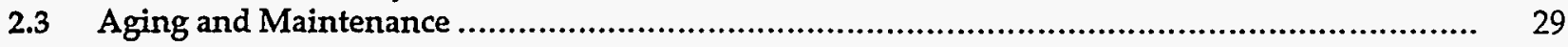

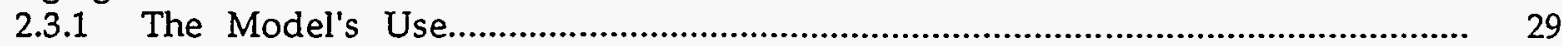

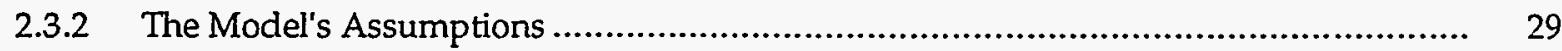

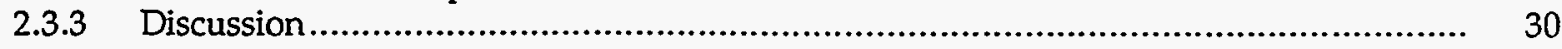

2.3.4 Comments on Degradation Modeling .......................................................... 36 


\section{CONTENTS}

2.4 Component Failure Rate Models and Reliability Distributions .................................... 38

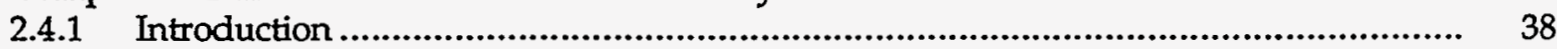

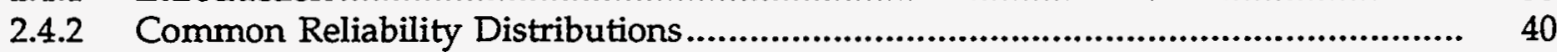

2.4.2.1 Exponential Distribution............................................................ 40

2.4.2.2 Normal Distribution ............................................................................. 40

2.4.2.3 Lognormal Distribution ..................................................................... 42

2.4.2.4 Weibull Distribution.......................................................................... 45

2.4.2.5 Extreme Value Distribution................................................................ 52

2.4.3 The Linear Aging Reliability Model (Component Aging Reliability Model) ............ 53

2.4.3.1 The Model's Objective ............................................................................ 53

2.4.3.2 The Model's Formulation.................................................................... 53

2.4.3.3 Assumptions......................................................................................... 54

2.4.3.4 Discussion..................................................................................... 56

2.5 Reliability Physics.................................................................................................. 56

2.5.1 Overview of Aging Mechanisms Affecting NPP SSCs............................................... 56

2.5.1.1 Fatigue............................................................................................ 59

2.5.1.2 Irradiation Embrittlement................................................................... 60

2.5.1.3 Stress-Corrosion Cracking................................................................... 62

2.5.1.4 Corrosion and Other Mechanisms........................................................... 63

2.5.2 Stress-Strength Interference Models......................................................................... 64

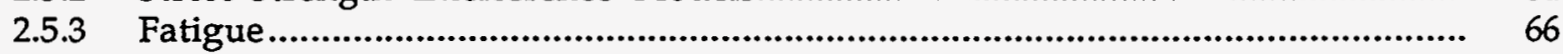

2.5.3.1 General Deterministic Models ................................................................ 66

2.5.3.2 Physical Fatigue Reliability Models and Comparison with the Linear Aging Model ................................................................................ 70

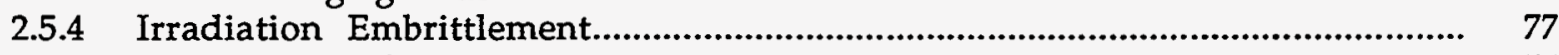

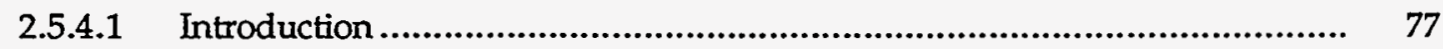

2.5.4.2 Correlations for the Ductile-to-Brittle Transition Temperature Shift...... 78

2.5.4.3 Pressure Vessel Failure Probability ....................................................... 79

2.5.4.4 Probabilistic Model for Pressure Vessel Reliability................................... 80

2.5.5 Stress-Corrosion Cracking....................................................................................... 81

2.5.5.1 General Deterministic Models ......................................................... 81

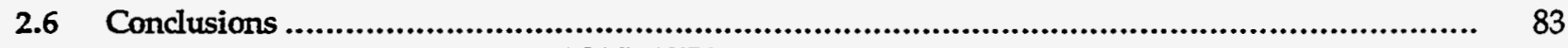

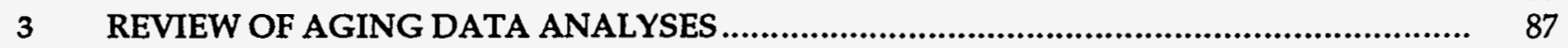

3.1 Data Used in the Prioritization and Selection of Systems, Structures, and Components

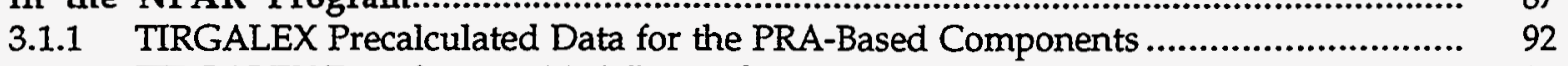

3.1.2 TIRGALEX Data for Non-PRA-Based Components ........................................... 92

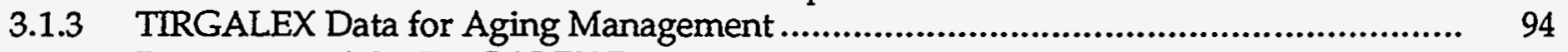

3.I.4 Discussion of the TIRGALEX Data ............................................................... 94

3.2 Operating Experience Used in Aging Assessments .................................................... 98

3.2.1 Generic Data Bases ............................................................................................ 98

3.2.1.1 Limitations on the Use of Generic Data Bases.............................................. 98

3.2.2 Plant-Specific Data................................................................................. 99

3.2.2.1 DORIAN: A Software Package for Bayesian Aging Data-Analysis ........... 99

3.2.3 Expert Opinions ............................................................................................. 100

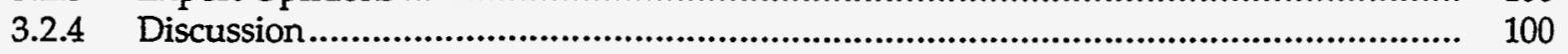

3.3 Data Used in Aging Assessments for Passive Components ............................................. 103

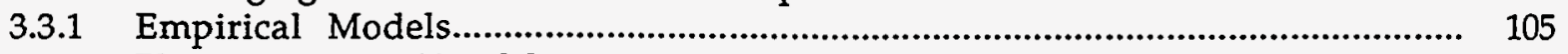

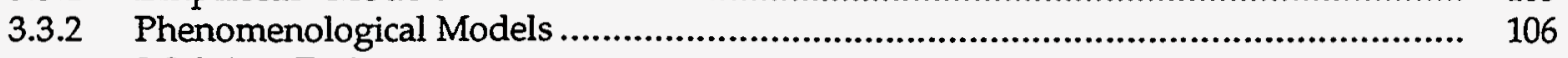

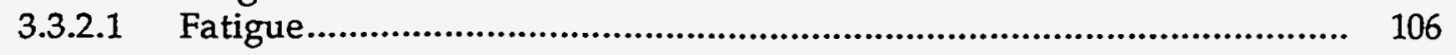




\section{CONTENTS}

Page

3.3.2.2 Irradiation and Thermal Embrittlement ....................................... 110

3.3.2.3 Erosion/Corrosion............................................................................ 111

3.3.2.4 Probabilistic Fracture Mechanics ........................................................... 111

3.4 Review of Statistical Methods Used in Aging Data Analysis .......................................... 114

3.4.1 Parametric Failure Rate Models ............................................................. 114

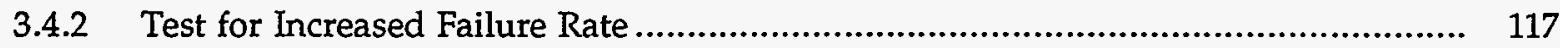

3.4.3. Test for Increased Failure Rate Average.................................................................... 118

3.4.4 Test for "New Better than Used" ...................................................................... 119

3.4.5 Alternative Methods of Failure Rate Estimation ....................................... 120

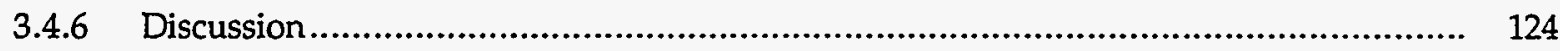

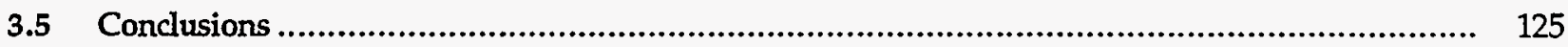

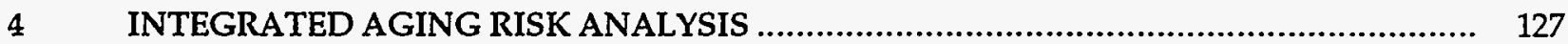

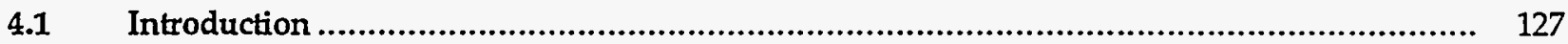

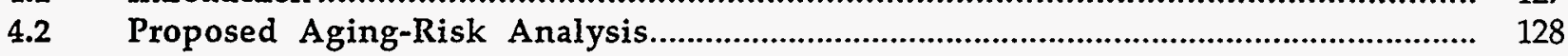

4.3 Selection of Aging Components and Aging Mechanisms .............................................. 130

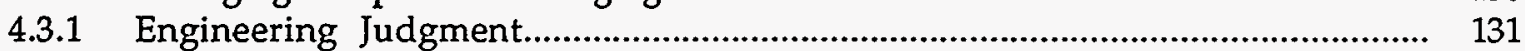

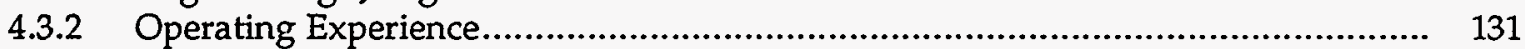

4.3.3 Risk-Significance Analysis .................................................................. 131

4.4 Probabilistic Physical Models of Aging Mechanisms ................................................. 132

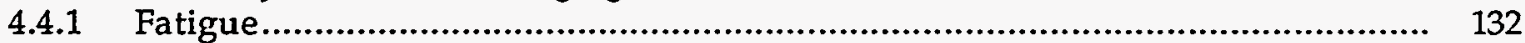

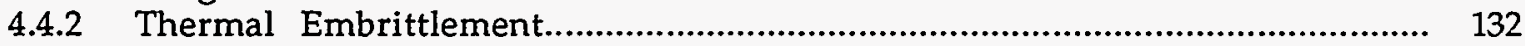

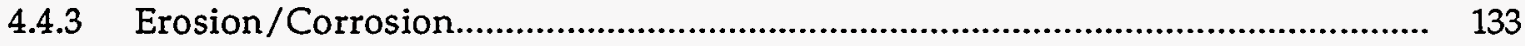

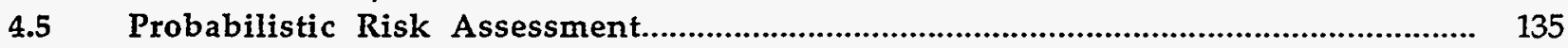

4.5.1 Effect on Core-Damage Frequency ....................................................... 136

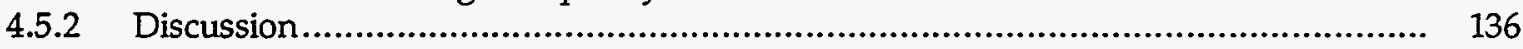

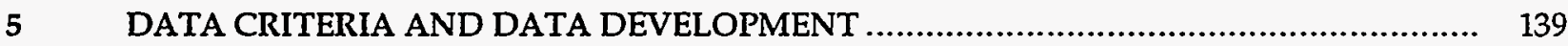

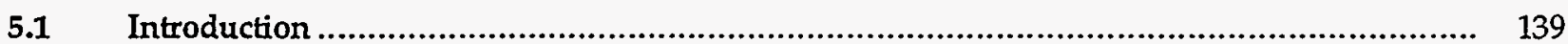

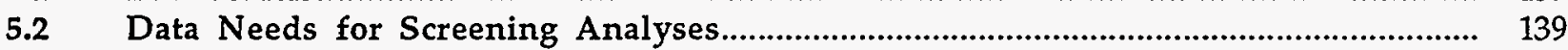

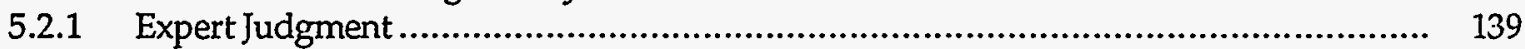

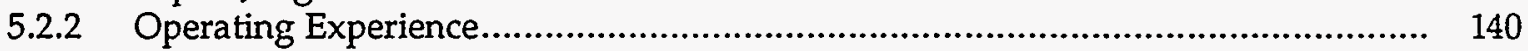

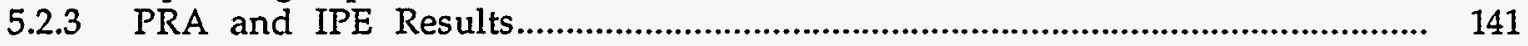

5.3 Data Needs for Modeling Aging Mechanisms .................................................... 141

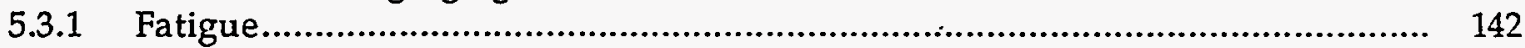

5.3.2 Irradiation Embrittlement........................................................................ 143

5.3.3 Stress-Corrosion Cracking and Erosion/Corrosion................................................. 145

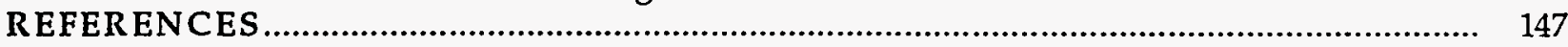




\section{LIST OF FIGURES}

\section{Page}

ES.1 - The PRAISE methodology (Harris et al., 1981) .................................................... xii

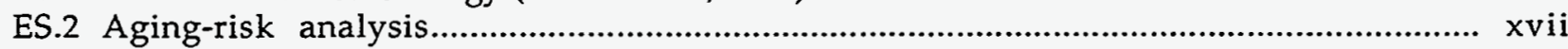

2.1 The PRAISE methodology (Harris et al., 1981) .................................................... 28

2.2 Markov state diagram (Samanta, 1991) ............................................................ 31

2.3 Transition probabilities (Samanta, 1991) .................................................................. 32

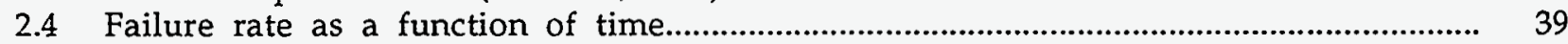

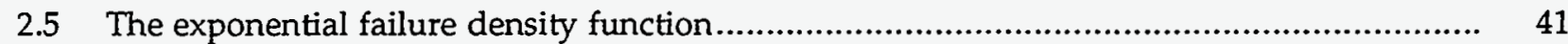

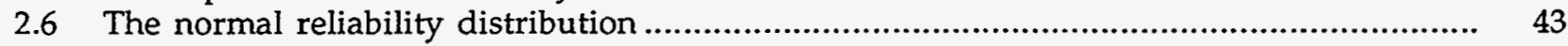

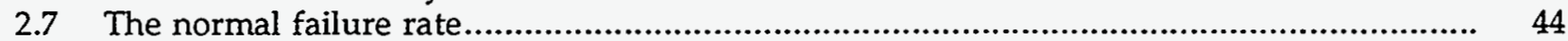

2.8 The lognormal failure density function.......................................................................... 46

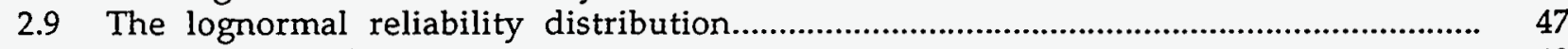

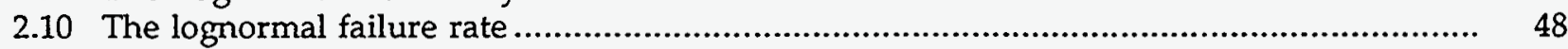

2.11 The Weibull probability density function for various values of $\beta$ when $\eta=1, \gamma=0 \ldots \ldots \ldots \ldots$.

2.12 The Weibull reliability distribution ......................................................................... 50

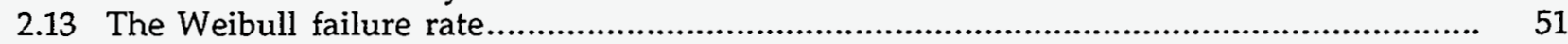

2.14 Effect of irradiation on the Charpy energy for a nuclear pressure vessel steel .................... 61

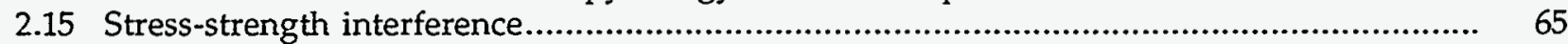

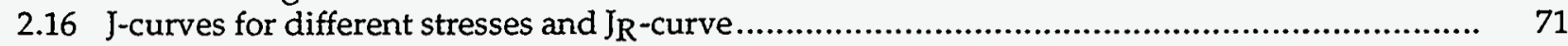

2.17 Approximate engineering strength-life relationship ............................................... 72

2.18 Fatigue and stress-corrosion cracking (Congleton and Craig, 1982) ................................... 82

2.19 Schematic representation of crack growth and time to failure (Wei, 1979)............................. 84

3.1 NPAR program outline (USNRC, 1991) ...................................................................... 88

3.2 The prior and posterior distributions of a component failure rate expressed in a set of linear aging models (Bier et al., 1991) ......................................................................... 101

3.3 Time-line data for a set of plant-specific motor-operated valves (Bier et al., 1990b) ........... 102

3.4 Stress-strength interference (SSI) increases with plant age (Phillip et al., 1992)................ 104

3.5 Pipe leakage causes by percentage ......................................................................... 107

3.6 The Bathtub Curve related to pressure vessels (Thomas, 1981) .................................... 108

3.7 Effect of $k_{c}, h, w, T, p H$, and $\mathrm{O}_{2}$ on pipe wall thinning .............................................. 112

3.8 Pipe diameter increase as a result of corrosion ........................................................ 113

3.9 Distribution of Weibull parameter estimate for various sample sizes $(\mathrm{N}) \ldots \ldots \ldots \ldots \ldots \ldots \ldots \ldots \ldots$

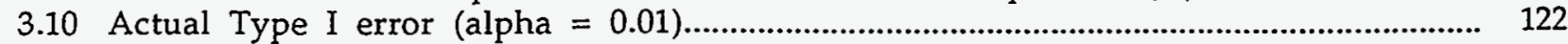

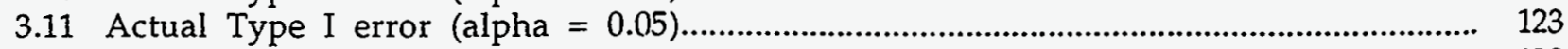

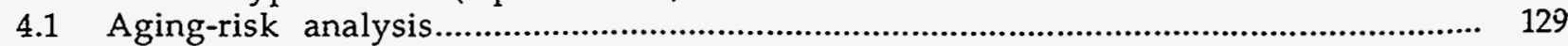

4.2 Evaluation of wall-thinning logic diagram (EPRI, 1988) ................................................. 134 


\section{LIST OF TABLES}

2-1 Main PWR components and their degradation mechanisms...

3-1 Factor values and final ranking of structures/components (Levy et al., 1988)..................... 93

3-2 Four-plant component risk importance data by component type (Levy et al., 1988) ............... 95

3-3 Results of nonparametric tests...................................................................................... 121 



\section{EXECUTIVE SUMMARY}

The Nuclear Regulatory Commission (NRC) initiated the nuclear power plant aging research (NPAR) program about six years ago to gather information about nuclear power plant aging. Since then, this program has collected a significant amount of information, largely qualitative, on plant aging and its potential effects on plant safety. However, this body of knowledge has not yet been integrated into formalisms that can be used effectively and systematically to assess plant risk resulting from aging, although models for assessing the effect of increasing failure rates on core damage frequency have been proposed.

The purpose of this review is to survey the work conducted to address the aging of systems, structures, and components (SSCs) of nuclear power plants (NPPs), as well as the associated data bases. The review takes a critical look at the need to revise probabilistic risk assessments (PRAs) so that they will include the contribution to risk from plant aging, the adequacy of existing methods for evaluating this contribution, and the adequacy of the data that have been used in these evaluation methods. A preliminary framework is identified for integrating the aging of SSCs into the PRA, including the identification of needed data for such an integration.

The major findings of this review are as follows.

- The issue of aging in NPPs cannot be addressed by models that are based solely on the current PRA structure and failure rates, such as the proposed plant-level and componentlevel models (NUREG/CR-5510 and NUREG/CR-4769). SSCs that the basic PRA model neglects as having very small failure probabilities and the principal degradation mechanisms, e.g., fatigue, embrittlement, and erosion-corrosion, must be considered.

- Probabilistic models for degradation mechanisms would allow the effective use of information regarding the aging of SSCs, which is similar to that collected by the NPAR program. Failure-rate-based models cannot accommodate this type of information, which typically does not include significant numbers of failures.

- Probabilistic models for the degradation mechanisms also would allow effective risk management strategies to be developed. For example, in probabilistic models for fatigue (Fig. ES.1), the crack nondetection probability is an important input; the effect of taking steps to improve this probability could never be assessed in the context of failure-rate models.

- The development of a methodology that includes aging mechanisms can build on existing PRA models, appropriately modified, as current external-event analyses do.

In Sec. 1, current PRA practice is reviewed to identify the underlying assumptions of plant modeling and data treatment methods. This review considers PRA plant and system modeling, eventtree and fault-tree modeling and the underlying hypotheses and estimation techniques and the underlying assumptions of component failure probability. Based on this review, the PRA models and assumptions that need to be reassessed when aging effects may be present can be summarized as follows.

(1) As a general practice, event-tree and fault-tree analyses of current PRAs do not include passive SSCs (e.g., numerous valve bodies, cables, reactor vessel supports, containment, piping, and the reactor vessel). The argument for doing so is that the failure rates for the passive SSCs are negligibly small. Although this argument may be reasonable when SSCs are new, a critical evaluation of this assumption is needed when the subjects of the investigation are older. 
(2)

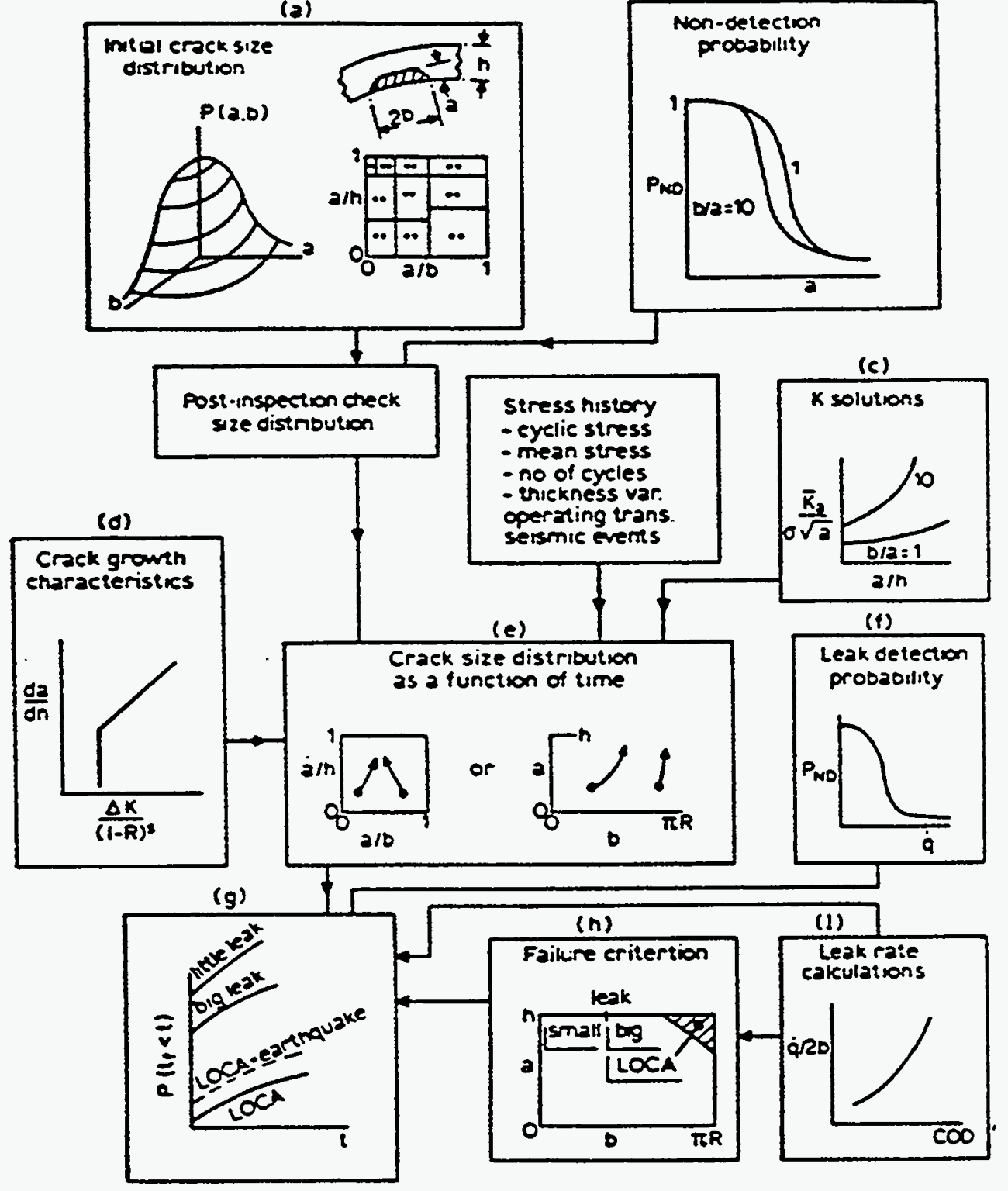

Figure ES.1 The PRAISE methodology (Harris et al., 1981). 
(2) The traditional PRA approach assumes a constant rate for each component failure mode, neglecting the possible effect of aging.

(3) Except for the failure rates of identical components that have been covered by commoncause failure analyses and by complete state-of-knowledge correlation, the current PRA methodology does not model other kind of dependencies. Aging may introduce additional correlations of failure rates even among dissimilar components.

(4) Failure probabilities of events other than hardware failures (e.g., pre-accident and postaccident human error rates, component unavailability because of testing and maintenance, etc.) could be different for older plants from those for new plants. The current PRA methodology does not make such a distinction, and this should be investigated further.

(5) External-event analyses need to be revisited. For example, aging may cause a shift in seismic fragility curves and the degradation of fire barriers.

In Sec. 2, the models and methods that are used to estimate the reliability of components and systems that are subject to age-related degradation are reviewed; the linearly increasing failure-rate model (Vesely, 1987) is the primary model reviewed. The principal question that is addressed is "Under what conditions, or in what time interval of an aging component's life, does a failure distribution derived from detailed physical models lead to a failure rate that can be approximated reasonably by a linearly increasing function?" To answer this question, we examine several models that have been proposed for three important degradation mechanisms (fatigue, radiation embrittlement, and stress-corrosion cracking). Each model is discussed and presented in terms of its objectives and its formulation. The assumptions made in the model formulation are presented, as is a commentary on whether these assumptions can be considered valid under general conditions and, if they are not, under which specific condition they still may be valid.

This review concludes that a linearly increasing failure rate model would not generally and unconditionally be applicable to many aging-related degradation mechanisms. Even in the case where the damage accumulates linearly with time, the derived failure rate is, in general, a complicated function of time rather than a simple linear function. Further detailed modeling of the specific damage-accumulation mechanisms, as well as the conditions that lead to failure, would have to be carried out to determine whether, and under what conditions, or in what time phase of the life of a specific class of components, the model of the linear failure rate remains a reasonable approximation. The currently available information is not sufficient for us to determine whether the linear model is a reasonable approximation in the life-time phase of interest for nuclear plant aging. For fatigue in particular, it is shown that, even though the damage accumulation resulting from fatigue may be (and commonly is in design applications) modeled as approximately linear, this model of linearly accumulated damage does not necessarily lead to a linearly increasing failure rate.

A major drawback of directly assuming a form for the failure rate (linear or not), rather than starting from the formulation of physical models for the degradation mechanisms, is that the underlying physical phenomena and parameters are not even identified in the analysis. This masking of the physics of the problem can affect aging risk management significantly. For example, working only with failure rates does not allow one to evaluate and understand the effect of an improved crackdetection probability on the reliability of a pipe. The proper way to do this would be to work with physical models that deal explicitly with crack growth.

The major advantage of the linear failure rate model is its simplicity and ease of application. If it could be demonstrated that this model is a reasonable approximation to the reliability of components experiencing age-related degradation, then it would be a very attractive choice. However, as stated earlier, for most of the degradation mechanisms of interest in nuclear plants, the true range of 
validity of the model has yet to be determined. Beyond the issue of the model validity in providing a means of estimating reliability and risk, a limitation would still remain in the model's inability to provide estimates of the effect of specific aging-management strategies on the probability of failure of components subject to aging mechanisms of a known physical nature.

The plant-level risk model proposed by Vesely in 1990 and 1992 uses Taylor-series expansions to incorporate aging effects into PRA. This model relates the change in individual component unavailabilities because of aging to the change in the overall plant risk (usually the core-damage frequency) through a Taylor-series expansion of the basic PRA model that calculates this risk from component unavailabilities. The results of the analysis are used to evaluate the effectiveness of maintenance and surveillance in controlling aging and to direct resources to those SSCs that are most important to NPP risk. Our review of this model has led to the following observations.

(1) Aging may increase the importance of factors that had been judged as negligible or had not been accounted for in the basic plant-risk model. Most important in this regard is the possible increase in the failure probabilities of major passive components, such as the reactor pressure vessel and the reactor coolant piping, and of passive elements of active components, such as pump casings and valve seats.

(2) Even though the model is presented in terms of changes in component unavailabilities, the only contribution to the unavailabilities currently considered is that resulting from changes in the failure rates. In fact, explicitly expressing the model in terms of failure rates, instead of component unavailabilities, would be more appropriate because various contributions to unavailability, e.g., those due to testing, would be explicitly included. In general, the failure rate would appear in several of these terms.

(3) Considering the effect of aging on risk through component unavailability is too restrictive because there are situations in which factors other than component unavailability may contribute to risk. For example, aging components (such as batteries) may reduce the available time for operator action during accidents, thus altering the probability of successful operator actions. More generally, any contributions that are analyzed based on the physics of the situation cannot be placed in this formalism. This includes seismic and fire analysis and Level 2 phenomena.

(4) The restriction to component unavailabilities and failure rates masks the physics behind the various contributors to risk, thus inhibiting risk management.

It should be noted that, notwithstanding the above observations, the idea of building on the existing PRA model to assess the effect of aging on plant risk is a good one and should be preserved as much as possible.

In Sec. 3, the data used in NPP aging analyses are reviewed. The data used in the NPAR program include generic and plant-specific operating experience, plant documents, test and research results, and expert judgments. The most extensive data base used in aging-risk analyses is the TIRGALEX (Technical Integration Review Group for Aging and Life Extension) data base, which was developed at a workshop held in May 1987 (Levy et al., 1988). This data base, which was used in studies by Vesely et al. (1990 and 1992) under the NPAR program, provides the input necessary to evaluate (1) the risk significance of component aging (RSCA) and, hence, a component contribution to plant risk because of aging; (2) the risk significance of component aging and aging management practices (RSCAAMP); and (3) the effectiveness of current management practices of the industry for maintaining an acceptable level of plant risk in the presence of aging components. The overall objective of the TIRGALEX program was to set priorities for the aging evaluation of structures and components. Based on our review, we have the following observations. 
(1) The TIRGALEX database was intended to provide more of a relative ranking of the aging features of the various equipment categories than absolute numerical values for aging of such SSCs. However, these numerical values are used extensively as they are an essential input in the linear-failure-rate model.

(2) The data base is the result of unstructured expert-opinion elicitation procedures and, as such, is subject to the well-known location and variability biases (in other words, the values reported may be widely off the mark). Uncertainty ranges are not reported.

(3) The data base is intended to be used as a generic data base that reflects industrial experience. However, in many cases, the TIRGALEX expert panel has based its judgment on a few plant-specific studies. Moreover, all numbers in the data base are assigned without statements on uncertainty, which may raise further concerns regarding the validity of its use for generic purposes.

(4) Although optimum test intervals, as well as the probability of successful detection of degradation and the probability of successfully mitigating the degradation given detection, depend on the specific aging mechanisms at work, no specific aging mechanisms are considered. This comment is related to our earlier observation, namely, that the use of failure rates alone masks the physics behind the various contributors to risk, thus inhibiting risk management.

The use of generic operating experience for identifying aging mechanisms also is reviewed in Sec. 3. There are four principal conclusions.

(1) No single data source (such as LER, NPRDS, IEEE-Std.500) provides the necessary information to perform a thorough analysis of component aging.

(2) Although a combination of various sources may allow a qualitative evaluation of the failure modes, causes, and aging mechanisms, it is difficult to reach any quantitative conclusions.

(3) In many cases, additional difficulties in the data analyses have been introduced because some vendors, utilities, and manufacturers have been reluctant to provide the necessary information.

(4) Data from operating experience are not sufficient, in quantity or quality, for validating any of the proposed aging models (linear, exponential, or Weibull), and the uncertainties in the estimate of failure rates, and especially in the rate-of-change of failure rates, remain high.

Very few analyses of plant-specific aging data have been conducted. One such analysis has been conducted by PLG Inc. to evaluate aging trends in equipment failure rates, maintenance rates, and maintenance duration. Data from maintenance records of two systems of a boiling water reactor (BWR) plant with $17 \mathrm{yr}$ of operating experience shows some interesting results (Bier et al., 1991). Although there is a slight tendency toward an increase in failure rates for some components, there is also a slight tendency toward a decrease in failure rates for some others. These rather ambiguous messages regarding the trend of component failure rates with time support our thesis that there is a need for deeper investigation of the underlying degradation mechanisms.

The existing physical models for aging mechanisms of passive components and the available data that pertain to these models also are studied in Sec. 3. In general, the data required for reliability physics based models of passive components consists of material properties and component stressors. The availability and quality of such data vary, depending on the particular component 
and/or mechanism being studied. However, for actual SSCs of interest in NPPs, almost no relevant data exist except for piping and pressure vessels.

Our review indicates that, with respect to aging mechanisms, there is a large amount of information available in qualitative form that comes from operating experience. This information is particularly important for passive components, where few actual failures have been observed, although degradation related to aging has been observed (Shah et al., 1987). However, the immediate usefulness of this information in terms of quantitative measures of reliability and risk is limited because this information is qualitative in nature. Nevertheless, it is valuable because it provides insights about which aging mechanisms are affecting which SSCs and the relative importance of these mechanisms.

Based on the observations we have made on aging-related issues, we conclude in Sec. 4 that to adequately address the overall risk at an aging plant, current PRA methodology needs to be extended. This extension would be accomplished most expediently if the effect of aging on plant risk were assessed within a framework that was built on existing PRA models. Such a framework is outlined in Sec. 4 , in which the effect of aging on plant risk would be analyzed in a separate section of the PRA. This method of treating aging mechanisms "externally" is similar to that for other external hazards (e.g., seismic events, fires, and floods). This framework, shown in Fig. ES.2, consists of three major parts: the selection of aging components and mechanisms, the physical modeling of selected components and mechanisms, and the integration of the findings from the first two parts into the PRA.

The analysis starts with the selection of a set of aging SSCs and degradation mechanisms that are significant to plant safety. It is necessary to limit the scope of the analysis to a manageable size because physical models, such as those of probabilistic structural mechanics, involve complicated formalisms and require detailed information. A large number of components would render such an evaluation approach ineffective. Technical bases for the screening process include engineering judgment, operating experience, and risk-significance analysis. This part of the analysis yields a selected set of components and mechanisms for further investigation.

The second part of the framework deals with developing physical models for estimating the failure probabilities of the selected components and mechanisms. Several such models could be suitable for our purposes (perhaps after suitable simplification), e.g., the software package PRAISE developed for the evaluation of failure probabilities resulting from component fatigue. For other aging mechanisms, such as erosion/corrosion, more work is needed to develop physical models more suited to reliability analysis. As shown in Fig. ES.2, the inspection, test, and maintenance practices at specific plants directly affect the time-dependent component failure rates. These practices and their effect should be modeled explicitly in the analysis.

The third part of the proposed framework integrates plant aging into the existing PRAs. Based on the findings from the screening analysis and the physical models, the initiating events, event trees, and fault trees in the PRAs will be reviewed and updated. The modification of PRAs may include the addition of basic events to fault trees and/or constructing new event trees and fault trees. This proposed framework retains most of the basic structure of current PRA models.

The availability and adequacy of the data required for the proposed framework for aging-risk analysis are discussed in Sec. 5. Data sources that can be used in the process of selecting aging components and mechanisms (the first part of the framework) include expert judgment, operating experience, and the results of existing PRA and IPE studies.

A future TIRGALEX-like expert panel and workshop also could be useful in conducting the screening analysis. A formal evaluation of expert judgment should include structured procedures in the following areas. 


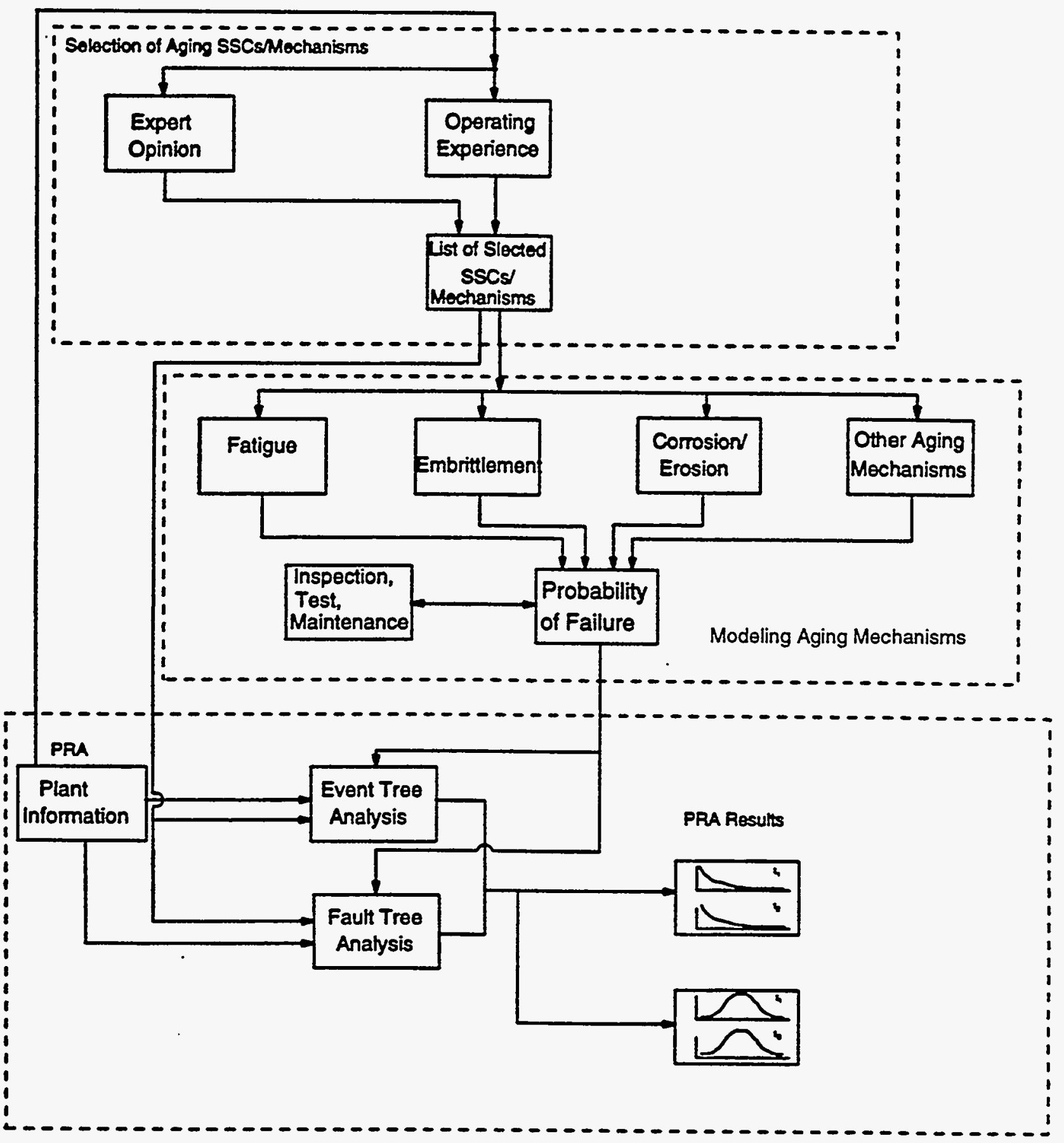

Figure ES.2 Aging-risk analysis 
(1) The selection of SSC aging-related issues about which the experts will be queried

(2) The identification and selection of experts

(3) The design and execution of the elicitation

(4) The treatment of biases of experts and biases induced during the elicitation process

(5) The dependency among experts

(6) The algorithms for aggregating expert judgments

(7) The treatment of uncertainty

In addition, the whole process of formal evaluation should be documented carefully. The qualitative reasoning process in support of the expert judgment constitutes an important portion of the elicitation process and also should be documented in detail.

Operating experience can provide two types of information: actual occurrences of failures caused by aging and evidence or direct observation of component degradation as a result of aging. Sources of operating experience include data from the In-Plant Reliability Data System (IPRDS) sponsored by the NRC, the Nuclear Plant Reliability Data System (NPRDS) managed by the Institute of Nuclear Power Operation, the Licensee Event Reports (LERs), plant maintenance records, in-service inspection reports and Nuclear Plant Experience (NPE) reports. Among these data sources, LER and NPRDS are the two most frequently used for aging analyses. The NPE data base is similar to that of the LER, whereas the IPRDS data base is very limited and incomplete.

When using generic data bases (such as NPRDS) in aging-risk analysis, one should be aware that they do not often contain information that is detailed and complete enough for drawing general conclusions. Events that are not of direct interest to the reporters are often overlooked or, at most, are roughly documented. The contents of the reports are often not detailed enough to allow aging-related root cause to be distinguished from other causes.

Plant-specific data generally contain more detailed information on the operational and failure history of specific systems and components. However, the major limitation of using plant-specific data in aging-risk analysis is that the number of incidents is often too small to reach a meaningful statistical conclusion, especially for plants that have only been in operation for a few years.

This situation could be improved by emphasizing the root-cause analysis in the existing data bases (such as LER or NPRDS). In particular, guidelines should be provided to the plant personnel regarding the reporting of aging-related equipment failures.

The results of plant-specific PRAs and IPEs can be useful for identifying components significantly affected by aging that are significant to plant safety. A recent study by Vesely (1992) on the prioritization of aging contributors and the evaluation of maintenance effectiveness is an example of such a use. As most PRAs and IPEs do not include passive components in their plant models, these would not be identified as risk-significant if the models were based on these data sources alone, even though some of these components might contribute significantly to aging risk. Consequently, it is important to note that the decisions made in the screening analysis should be based on sources broader than the PRA and IPE results.

Also in Sec. 5, an assessment of the availability and adequacy of the data needed for the development of models for the aging mechanisms (the second part of the framework) discussed in Sec. 2 
is conducted. Physical probabilistic models for estimating aging effects on component failure rates often require a large amount of data related to material properties and operating conditions. In the review of degradation mechanisms and associated models performed in Sec. 2, a wide range of data sources is identified.

For the aging mechanisms of fatigue and irradiation embrittlement, it is found that there is a wide availability of data related to the material properties associated with the physical modeling of these aging mechanisms. However, much of these data are presented in a conservative, deterministic framework, and it may be necessary to recast much of it in a statistical format so that probabilistic evaluations of the aging mechanisms can be performed.

For fatigue, there are a couple of areas for which the available data may not be appropriate for component and plant-specific reliability analyses. Special care should be taken when selecting crack-growth data, which can be affected by environmental effects such as corrosion. Therefore, the environmental conditions under which a component is operating should be assessed to determine whether the available crack-growth data are applicable and, if not, further research may be necessary to provide data that is appropriate. Also, more crack-growth data in the ultra-low growth-rate regime below $10^{-6} \mathrm{~mm} /$ cycle, approaching the so-called fatigue threshold, may be needed because fatigue lifetimes for some components can be dominated by crack extension in this region.

With respect to radiation embrittlement, it appears that, for most plants, the surveillance program provides an adequate supply of raw data to characterize the fracture mechanics parameters for a plant-specific vessel reliability study. However, for a probabilistic treatment of irradiation embrittlement, it may be necessary to convert the fracture-toughness data base into corresponding statistical data for stress intensity factors. Other areas of concern include the characterization of an initial flaw distribution and the extrapolation of neutron exposure data from the surveillance capsule locations to the vessel wall. Flaw distributions that are used in the probabilistic fracture mechanical (PFM) analysis portion of a pressurized thermal shock (PTS) risk assessment can affect the overall results by several orders of magnitude, and thus, care must be taken to use a technically appropriate flaw distribution when dealing with an integrity assessment of vessels with low upper-shelf toughness behavior.

Stress-corrosion cracking and erosion/corrosion are two damage mechanisms much less quantified and analyzed than fatigue and embrittlement. Data and models related to stress-corrosion cracking and erosion/corrosion are mainly of fundamental, rather than engineering, value, and thus, it is concluded that both data collection and modeling research are needed before reliability analyses of components affected by these mechanisms can be conducted. 


\section{REVIEW OF PRA METHODOLOGY}

\subsection{Introduction}

This report describes the assumptions made in the probabilistic risk assessment (PRA) of a nuclear power plant (NPP). Numerous assumptions are made in these PRAs, both within the standard PRA methodology and on plant-specific situations. However, the focus of this report is on the generic assumptions made in the PRA methodology regarding the following items: accident sequence identification, system models, sequence quantification, and external events analyses, especially seismic analysis. To provide necessary background information, each section starts with a brief description of the models used in PRAs. This is followed by a detailed discussion of the major assumptions made in these models and a comparison of major differences between assumptions made in different models. General reference material and PRA studies cited in this report include the Reactor Safety Study (USNRC, 1975), the PRA Procedure Guide (USNRC, 1983), NUREG-1150 (USNRC, 1990; Breeding et al., 1992), the Beaver Valley IPE (PLG, 1990), the Savannah River PSA (Westinghouse, 1990), the Diablo Canyon IPE (PG\&E, 1992), and the LaSalle PRA (USNRC, 1992).

\subsection{Accident Sequence Identification}

\subsubsection{Event-Tree Analysis}

The PRA Procedures Guide (USNRC, 1983) gives an overview of the PRA methodology showing how accident sequences are identified. Another article describing the overall approach of event-tree analysis is by Kaplan (1981). To keep the analysis manageable, a common practice is to model the plant, the containment, and the site separately and to connect them through "pinch points." This allows analysts to break up the PRA effort when separate analyses are feasible. One can conveniently think of initiating events as the front end of a PRA and the final consequences at the site as the back end. Between the initiating events and the final consequences, two sets of pinch points are used in a standard PRA: plant damage states and release categories.

An event-tree analysis starts with the identification of the initiating events. This is often done by applying analytical methods such as a master logic diagram and a failure modes and effects analysis (FMEA). The master logic diagram is a deductive approach for directly addressing the question "How can a significant release of radioactivity to the environment occur?"

When the initiating events have been identified, event trees and required fault trees are constructed for each group of initiating event. An event tree connects an initiating event with plant damage states. Systems and components used to model the accident-sequence progression are placed along the top of the event tree. The assumed success or failure of these systems leads to multiple eventtree branches that result in specified plant damage states. The event-tree branches occur at branch points that specify whether or not the system or component along the top of the event tree is functioning.

The quantification of the frequency for plant damage states is of primary importance in a PRA and is carried out by developing fault trees for the systems and components along the event-tree top. Two approaches to event-tree development and quantification have evolved over time: the large event tree/small fault-tree (LET) approach and the small event-tree/large-fault tree (SET) approach (Rasmuson, 1992). Preference as to which one is used seems to be primarily one of analyst choice and which codes are available. Both approaches should give essentially identical results if the systems and components are modeled correctly. Rasmuson (1992), identifies a variety of attributes, pro and con, for each approach. In the LET approach, support systems and front-line systems modeled as trains are used as event-tree top events. In the SET approach, front-line systems are used as event-tree top events, 
with support systems being included in the fault trees. This difference in modeling manifests itself in where system dependencies are found. In the LET approach, these dependencies are in the event trees, whereas in the SET approach they are in the fault trees. Placing the dependencies in the event tree allows (ideally) for independent fault trees and a straightforward plant damage frequency calculation but obviously creates very large event trees. When the dependencies are placed in the fault trees, plant damage state frequencies must be calculated via cut sets. Computer codes exist that allow both large event and large fault trees to be constructed and quantified (Stack, 1984; Worrell, 1985; SAIC, 1988; Russel et al., 1991)

Between plant damage states and release categories, the progression of accident sequences is modified by a containment event tree. The release categories are end states on a containment event tree. Each of the release categories specifies a certain quantity and mix of radioisotope release. After the release of radioactive material from the containment, the site model, which includes consequence analysis and health effects models, is used to determine the final consequences of a specific release category. An accident-sequence progression analysis at these later stages can be carried out by using a variety of codes. These codes include MELCOR for accident sequence progression (Summers et al., 1991), CONTAIN for containment modeling (Murata et al., 1989), and the MELCOR Accident Consequence Code System (MACCS) for consequence analysis (Chanin et al., 1990).

Most of the assumptions made in the event-tree analysis are plant-specific and are related to the physical arrangement of the systems. However, several underlying assumptions are made in the event-tree modeling.

(1) It is assumed that the states that a system could be in are discrete and finite. With only a few exceptions, success and failure are the only two states that are modeled in the PRA. This binary expression sets a constraint on the event-tree model for analyzing deteriorated systems.

(2) Event-tree modeling is adequate for events that do not involve too much feedback. For dynamic situations where feedback is essential to the progression of the events, the event tree oversimplifies reality.

(3) In the LET approach, it is assumed that all the physical dependencies between systems are identified by the event analysts, whereas the dependencies are identified when solving the fault trees using a computer code in the SET approach.

\subsubsection{System Analysis}

All reactor PRAs use event-tree and fault-tree analyses (Vesely et al., 1981) to perform system analysis. One major assumption made in the fault-tree analysis is that the states of components and systems are binary (i.e., either success or failure). Because of this, fault-tree analysis is not an ideal model for degraded components or deteriorated systems.

Certain assumptions must be made in modeling any physical system. Because the fault trees and event trees for a nuclear plant are models of system behavior under various conditions, an analyst must make certain assumptions. There is no given standard list of assumptions, and each analyst, or group of analysts, must develop their own specific list of assumptions. Although there are general guidelines for developing event trees and fault trees (USNRC, 1983; Vesely et al., 1981; Carlson et al., 1983), the final models for plant systems, structures, and components (SSCs) and their interactions are based on assumptions. These assumptions are required because the analyst is

(1) uncertain about the actual mechanistic behavior of a specific system under certain conditions (e.g., fuel/coolant interaction); 
(2) uncertain about the interaction between a given system and other systems, both human and electro-mechanical (e.g., human action during an emergency);

(3) uncertain about the parameters characterizing the behavior of the system under certain conditions (e.g., component failure rate); and

(4) uncertain about, or incapable of modeling, actual system behavior because of model implementation (e.g., computational) difficulties.

The degree of sophistication used in developing models depends on the analyst's knowledge, experience, and skill. Practical constraints such as funds available, computational limits, and time to perform the analysis are also important. Another important practical consideration is the availability of system data.

We briefly discuss some assumptions made in the system analysis for a PRA in the following sections.

\subsubsection{Scope of the System Analysis}

The following assumptions are made for the scope of the system analysis.

(1) For the fault analyses of most PRAs, only those components necessary to quantify system unavailability for the specified boundary conditions are modeled. As a result, passive components, the failure probabilities of which are considered negligible, are not modeled. As a general rule, passive failures (e.g., the failure of a normally closed valve to remain in a closed position) also are not considered in the model unless that failure could contribute significantly to individual loop or system unavailability.

(2) The level of detail in modeling components often is chosen to be consistent with the best available equipment data. For example, the models for motor-operated valves do not specifically identify individual circuit breakers, torque switches, and limit switches because the best available data for valve failures include all subcomponents of the valve assembly and its motor operator. Similarly, relay models do not separately identify individual contact failures, and electronic circuit models are developed only to the level of replaceable modules or major circuit boards.

(3) The boundaries and scope of front-line system models are generally the same as those found in plant system-engineering descriptions. However, because of their broad effects and the interrelationships among components, the modeling boundary and scope of some support systems may be defined slightly differently than in normal engineering and design descriptions to facilitate efficient model integration. Below are three common modeling assumptions on systems boundaries that are generally made in PRAs.

(a) Electrical boundaries for mechanical and fluid systems are generally made between the bus or panel containing the supply circuit breaker and the breaker itself.

(b) The boundaries between actuated systems and the solid-state protection system (SSPS) are often made at the point where the commonality ends. For example, a relay that is common to the actuation of several systems is modeled as part of SSPS, and the successive relays are modeled as part of the actuated systems.

(c) The boundaries between cooling-water systems and the systems that they support are often made so that the analysis includes all components needed to support more than one other system (generally including pumps, headers, and header isolation valves). 
A failure of cooling-water-system components that support only one other system is included in the analysis of the supported system. Examples of these components are branch lines to individual cooling loads, cooling-load isolation valves, and individual load-cooling coils. However, piping or valve failures that might disable an entire train of a support system or impact more than one analyzed system, if their failures are modeled, are included in the cooling system.

(4) The level of detail of the system analysis depends on the objective of the analysis. For example, in the LaSalle PRA (USNRC 1992), the objective of which was to integrate external events into a common framework with internal events, the level of detail of the fault-tree models was extended to include components that would be affected by, and failure modes that might be induced by, external events. As a result, the LaSalle PRA includes the modeling of (1) components such as piping and cables, which would not normally be included in an internal event PRA because their passive failure probability would be very low; (2) control and actuation circuitry in detail to represent the effect of the external events on the system accurately; and (3) failure modes such as spurious actuation. Also, the methodology of the LaSalle PRA allows the analysts to map the location of all components modeled in the fault trees (including the tracing of all cables and pipes). This allowed the evaluation of location-based failures (induced by events such as fire and flooding) and the simultaneous inclusion of multiple random failures consistent with the overall probabilistic truncation probability.

\subsubsection{Qualitative System Analysis}

Some of the qualitative analysis of a specific system is conducted before the analysts physically start constructing fault trees. The analysis starts with a system screening process that determines the response of each system to normal and transient operating conditions. The combinations of all possible system successes and failures characterize the possible responses of the plant to the initiating events. In the screening process, each system was categorized as either a "support" system or a "front-line" system. It was also determined whether further analysis of a system was necessary. The following information is collected for each system.

- Function

- Success criteria

- Support required for system to perform function

- Supported system

- Operation and special features

- Testing

- Maintenance

- Recovery considerations

- Potential for event initiation

- Technical specification requirements

- Modeling assumptions 
- System logic model

- Reactor is assumed to be at full power

- Sabotage is not included

The sources of the information are plant documents such as the Final Safety Analysis Report (FSAR), system descriptions, plant procedures (maintenance, test, operations), and drawings (e.g., electrical schematics, logic drawings, and piping and instrumentation drawings).

The system success criteria for the study are derived from the FSAR. In the case of exceptions (where FSAR criteria are not directly applicable or are unrealistically conservative), success criteria are developed by applying engineering judgment based on documented analyses and previous probabilistic risk assessments.

The qualitative system analysis also includes the definition of the modeling assumption for the fault-tree analysis and the quantitative analysis to be executed next. Some general modeling assumptions that are applicable to most system models are as listed below.

(1) The important plant systems are assumed to be operated and maintained in accordance with the plant technical specifications except for explicitly modeled events such as system misalignments and maintenance errors.

(2) A mission time of $24 \mathrm{~h}$ is assumed for most systems in most PRAs. This provides a sufficient time base on which accident progression can be measured and provides a realistic and consistent time in which outside actions could be started to prevent later (after $24 \mathrm{~h}$ ) core damage.

(3) Plant systems are assumed to be operated and maintained in accordance with the current written operating and maintenance procedures.

(4) If pipe breaks are modeled in the system analysis, they are considered only if the break by itself can cause the system to fail. Pipe breaks at the plant level are considered in the internal flooding analysis.

(5) Relief valve leakage or premature opening is considered only if it is deemed possible to divert sufficient flow to defeat the train or system.

(6) Vents and drains are not modeled for flow diversion because of their small leak sizes and because they are normally closed.

\subsection{System Quantification}

The most often displayed logic expression for system unavailability in PRAs is a disjunctive normal form (i.e., sum of products of basic events) of minimal cut sets. Each minimal cut set consists of the minimal number of basic events whose concurrence leads to the failure of the system. Therefore, system unavailability is estimated by quantifying these minimum cut sets. During the quantification process, certain information is needed about each basic event. When uncertainty in the parameters (e.g., failure rates) of basic events is taken into account during the system quantification, the correlation between the state of knowledge of the parameters must be treated properly. It has been shown (Apostolakis and Kaplan, 1981) that some substantial errors can result from the improper treatment of correlation among parameters. These issues are discussed in the following sections. 


\subsubsection{Basic Events}

In a data analysis of an internal PRA analysis, the following parameters are estimated before the system quantification.

(1) Component failure rates

(2) Common-cause factors (CCFs)

(3) Pre-accident human error rates

(4) Component unavailability because of test or maintenance

The models for these basic events used in the PRA data analysis are discussed below.

\subsubsection{Component Failure Distribution}

Two basic probability models are used in PRAs for component-failure modeling: exponential distribution for time-related failures and binomial distribution for demand failures. This section discusses the assumptions made in these two models.

\section{Time-Related Failure Model}

The failure of a component that operates continuously (e.g., pumps) usually is modeled as an exponential distribution having a failure rate, $\lambda$. The cumulative failure distribution function, $F(t)$, can be expressed in the form

$$
F(t)=1-\exp (-\lambda t)
$$

and the failure density function, $f(t)$, is

$$
f(t)=\lambda \exp (-\lambda t)
$$

For a time-to-failure distribution that has a density function $f(t)$, the failure rate (or hazard function) is defined as

$$
\lambda(t)=f(t) /[1-F(t)]
$$

Thus, the failure rate is defined as the conditional probability of a component that fails between time $t$ and $\mathbf{t}+\mathrm{dt}$ (where $\mathrm{dt}$ is a small time interval), given that the component is functioning at least up to time $t$.

Three basic assumptions are made when an exponential distribution is used.

(1) Binary Representation. Only two states, success and failure, are considered for the outcome of each demand.

(2) Stationarity. The failure rate, $\lambda$, of an exponential distribution is constant over time. At any time, $t$, the conditional probability that the component will fail within the next time interval, $\mathrm{dt}$, given that the component survives until time, $t$, is independent of $t$. In other words, the exponential distribution assumes that the component does not deteriorate as time goes by. 
(3) Relationship to the Poisson Distribution. Poisson distribution gives the probability of $r$ events occurring in an interval $(0, T)$ given that their rate of occurrence is constant, $\lambda$ :

$$
\mathrm{p}(\mathrm{r})=\exp (-\lambda \mathrm{T})(\lambda \mathrm{T})^{\mathrm{r}} / \mathrm{r} !
$$

exponential distribution can be written as

$$
F(t)=1-p(0),
$$

where $p(0)$ is the Poisson term for zero failures. This leads to the following interpretation: if failures occur because of lethal shocks occurring according to the Poisson process, the component will survive as long as no shocks have occurred.

The exponential distribution is often approximated by

$$
F(t) \approx \lambda t \text {, for } \lambda t<0.1
$$

This is a very common approximation because $\lambda t<0.1$ in most applications. However, this condition is not always satisfied, as Apostolakis and Kaplan (1981) have shown.

\section{Demand-Failure Model}

For most standby components, the failure of a component only occurs when it is switched on line. The binomial distribution is the most commonly used failure-on-demand model in PRA studies. The probability that there are $r$ failures out of $n$ demands is given by the binomial distribution: where $p$ is the relative frequency of failure per demand. The underlying assumptions of a binomial distribution are

$$
p(r)=\left(\begin{array}{l}
n \\
r
\end{array}\right) p^{r}(1-p)^{n-r}
$$

(1) Binary Representation. Only two states, success and failure, are considered for the outcome of each demand.

(2) Bernoulli Trials. The probability of success or failure on each demand, p, remains constant from demand to demand.

\subsubsection{Common-Cause Failures}

Minimal cut sets are quantified by taking the product of the probabilities of the basic events which are assumed to be completely independent. "Common-cause events" in PRAs are a subset of dependent events in which two or more component fault states exist at the same time (or in a short time interval) and are a direct result of a shared cause. To incorporate common-cause events into the systems analysis, the analyst must understand the factors that determine the dependence or independence among the components in the system. Such factors include how groups of components are used, the extent of their diversity (if any), the physical proximity or separation of redundant components, and the susceptibilities of system components to varied environmental stresses. Similarity in design, manufacture, and model among components of different trains implies the potential existence of strong dependence. Common-cause effects currently are assumed in PRAs not to exist among dissimilar equipment, although research exists on broader issues of common cause effects such as organizational and managerial impacts (Wu et al., 1989). 
Numerous models have been proposed for the treating common-cause events, for example, the alpha-factor model (Mosleh and Siu, 1987), the beta-factor model (Fleming, 1975), the multiple-Greek letter (MGL) model (Fleming et al., 1986), the binomial-component failure-rate (BFR) model (Vesely, 1977), the multiple failure-rate (MFR) model (Apostolakis and Moieni, 1987), and the stochastic reliability analysis model (Dorre, 1990). Among these models, the beta-factor and the MGL models are used most often in the existing PRAs. More discussion on the models used for common-cause events can be found in Mosleh et al. (1988) and Mosleh (1990). The parametric models (e.g., the MGL and beta-factor models) and shock models (e.g., the MFR models) are discussed below, and the differences between the major assumptions for each are elucidated.

\section{Multiple Greek Letter (MGL) Model}

The MGL (Fleming et al., 1986) model deals with the effect of common-cause events on different levels of redundancy. Greek letters are used to represent the effect of common-cause events on different numbers of components. For a common-cause component group that has $\mathrm{m}$ redundant components, $\mathrm{m}$ parameters are defined in the MGL model. For example, when $\mathrm{m}=4$,

$$
\begin{array}{rr}
(1-\beta) \lambda_{t}, & i=1, \\
\beta(1-\gamma) \lambda_{t}, & i=2, \\
\lambda_{c, i}=\gamma \beta(1-\delta) \lambda_{t}, & i=3, \\
\delta \gamma \beta \lambda_{t}, & i=4,
\end{array}
$$

where $\lambda_{c, i}$ is the random failure rate when $i=1$ and the common-cause failures rate when $i$ is greater than 1. The total failure rate of the component is $\lambda_{t}$. The parameters of the MGL model for the case of simultaneous testing are estimated from the number of demands, $N_{D}$, and the number of the commoncause events of $k$ failures at each event. For the case that $N_{D}$ cannot be estimated from plant records, $\lambda_{t}$ can be obtained from other reliability data sources.

The following assumptions are made in the MGL model.

(1) The failure rates of common-cause events are assumed to be proportional to the failure rate of a component.

(2) It is assumed that the parameters ( $\beta, \gamma, \delta$, etc.) in the MGL model can be estimated from generic data sources and plant-specific experience. When data on multiple failures are scarce in both industrial and plant-specific domains, the estimation of these parameters may depend heavily on subjective judgment.

(3) Only dependencies between identical components are treated in these models. Causes such as high room temperature, which may have an effect on components of different kinds, or aging, which may have an even broader effect over multiple systems and components, are not dealt with in these models.

(4) The parameters of the model are treated as uncorrelated variables even though they typically are determined from similar sources of information. 


\section{Beta-Factor Model}

In the beta-factor model (Fleming, 1975), the fraction of component failures associated with common-cause events is denoted by $\beta$, and the total failure rate of a specific component is denoted by $\lambda_{t}$. The common-cause failure rate, $\lambda_{c}$, of the component group is then

$$
\lambda_{\mathrm{c}}=\left[\begin{array}{ll}
(1-\beta) \lambda_{\mathrm{t}}, & \mathrm{k}=1, \\
0, & 1<\mathrm{k}<\mathrm{m}, \\
\beta \lambda_{\mathrm{t}}, & \mathrm{k}=\mathrm{m} .
\end{array}\right.
$$

To estimate $\lambda_{t}$ and $\beta$ from the plant records, one needs to know the number of components in the commoncause component group, $m$, the total number of demands of the component, $N_{D}$, the number of random failures in the records, $n_{1}$, and the common-cause failures that involve $k$ components, $n_{k}$.

In addition to the assumptions made in the MGL model, there is an additional assumption made in the beta-factor model that whenever a common-cause event occurs, all components within the same common-cause component group fail at the same time. For example, if the four motor-operated pumps in the emergency-service water system are considered as a common-cause component group, then all four pumps would fail at the same time when a common-cause event occurs. This often results in an overconservative estimate on the common-cause failure rates.

It can be easily seen that the MGL model is an extension of the beta-factor model which, in general, gives conservative estimates on multiple failures because of its assumption that all fail if one fails. Models like MGL model are developed to somewhat relax this assumption.

\section{Multiple Failure-Rate (MFR) Model}

Several fundamental modeling assumptions are made in the MFR (Apostolakis and Moieni, 1987) model for a system of $m$ identical components.

(1) It is assumed that two types of failures are associated with a component: random failures, $\phi_{I}$, that result from independent causes and common-cause failures from shocks that may cause any number of components to fail at the same time.

(2) The shocks that may cause more than one component to fail occur at a constant rate, $\mu$.

(3) Given the occurrence of a shock, there is a fraction of $\phi_{i}$ that exactly i components fail after the shock. It follows that

$$
\sum_{i=0}^{m} \phi_{i}=1 .
$$

Unlike the MGL model, this model further allows the modeling of state-of-knowledge dependence to be reflected in the vector of parameters $\phi=\left(\phi_{\mathrm{I}}, \mu, \phi_{0}, \ldots, \phi_{\mathrm{m}}\right)$. In the paper (Apostolakis and Moieni, 1987), beta and Dirichlet distributions are assigned to the state-of-knowledge distributions of parameters $\left(\phi_{1}, \mu\right.$ and $\left.\phi_{i}{ }^{\prime} s\right)$. Unlike the MGL method, this model has not been used in PRAs. 


\subsubsection{Pre-Accident Human Errors}

Examples of pre-accident human errors are errors involved in restoring a component or flow path to its normal operating status after testing, inspection, or maintenance activities are completed. These activities are usually system-specific and are performed routinely by one or more individuals as part of the normal workday duties. These activities are not directly related to operator actions or equipment response during a plant transient, although errors in the performance of these actions may leave important equipment disabled and require additional recovery actions to restore it to service during the transient. The routine testing, maintenance, and surveillance actions are evaluated separately and incorporated into the system analysis as specific basic events that cause equipment to become inoperable.

The most often used model for pre-accident human errors is the THERP (technique for human error rate prediction) model described in the NRC Handbook of Human Reliability Analysis (Swain and Guttmann, 1983). In THERP, a task analysis is performed to provide a description of the performance characteristic of the subject task. The results of the task analysis are represented in a human-reliability-assessment (HRA) event tree. A set of pre-established nominal probabilities are modified to allow for the effects of event-specific performance shaping factors (PSFs), such as operator experience, stress level, and control room display. Human error probabilities then are estimated through the analysis of the HRA event tree. Some of the assumptions made in the THERP model are as follows.

(1) It is assumed that the subject human task can be broken into subtasks to which probabilities can be assigned. This subdivision of tasks is not always feasible, especially for actions that are dynamic or mission oriented. For pre-accident human activities where subtasks are more or less well defined, THERP is a widely used model in the PRA community.

(2) The model assumes that there exists a credible data bank, such as the NRC Handbook, for assigning failure probabilities to human errors under various operating environments.

(3) It is assumed that the PSFs in each subtask are identifiable and measurable.

(4) It is assumed that the nominal failure probabilities can be modified easily to include PSFs.

\subsubsection{Component Unavailability Because of Test and Maintenance}

The component unavailability because of test or maintenance is estimated by taking the product of the test (or maintenance) frequency and the mean test (or maintenance) duration (or mean time to test or to repair):

$$
\mathrm{U}_{\mathrm{i}}=\mathrm{f}_{\mathrm{i}} * \tau_{\mathrm{i}}
$$

where $U_{i}$ is the unavailability of component $i$ because of test (or maintenance) activities, $f_{i}$ is the frequency of test (or maintenance), and $\tau_{\mathrm{i}}$ is the test (or maintenance) duration. This approach of component unavailability because of test or maintenance includes two assumptions.

(1) In the component test-and-maintenance unavailability model, both test (maintenance) frequency and mean duration of test (maintenance) of all components remain constant over time.

(2) Components are as good as new after each test and maintenance activity. 


\subsubsection{The Quantification of System Unavailability}

System unavailability is estimated by quantifying minimal cut sets and their conjunctions. This section discusses the models and assumptions used in quantifying system unavailability: the occasion of rare events, the system unavailability under periodic test and maintenance, and the treatment of parametric correlations.

\subsubsection{System Unavailability Under Periodic Test and Maintenance}

An analytical model for the unavailability of safety systems under periodic test and maintenance is presented in Apostolakis and Chu (1980). The engineered safety systems in nuclear power plants are installed for emergencies and are normally on standby. To ensure their operability, they are tested and, if necessary, repaired at periodic intervals. The pointwise (time-dependent) system unavailability, $q(t)$, is defined as the probability that the system is down at time $t$. The average unavailability, $q_{a v}$, over time interval of interest $(0, T)$ is defined as

$$
\mathrm{q}_{\mathrm{av}} \equiv(1 / \mathrm{T}) \int_{0}^{\mathrm{T}} \mathrm{q}(\mathrm{t}) \mathrm{dt}
$$

\section{Average Unavailability of Single-Component Systems}

The analytical expression for the average unavailability of a single standby component that is tested periodically is given as

$$
\mathrm{q}_{\mathrm{av}}=\left(\tau_{\mathrm{r}} / \tau\right)+\gamma+\mathrm{Q}+(1 / 2) \lambda \tau
$$

where

$\tau=$ interval between tests,

$\tau_{\mathbf{r}}=$ duration of test,

$\gamma=$ probability of failure due to testing,

$Q=$ probability of failure on demand, and

$\lambda=$ failure rate (the failure distribution is assumed to be exponential).

The various contributions to the average unavailability are

$$
\begin{aligned}
&\left(\tau_{\mathrm{r}} / \tau\right)= \text { test contribution (the component has been assumed to be disabled during testing), } \\
& \gamma=\text { human error contribution (testing), } \\
& Q=\text { contribution from failure on demand, and } \\
&(1 / 2) \lambda \tau=\text { contribution from "random" failures occurring between tests while the component is } \\
& \text { on standby. }
\end{aligned}
$$


The average unavailability of a one-out-of-two system under a sequential testing scheme can be represented by the sum of four terms:

$$
q_{a v}=q_{R}+q_{C}+q_{D}+q_{M}
$$

where each term is further defined as (1) a "random" independent failure contribution,

$$
\mathrm{q}_{\mathrm{R}}=(1 / 3) \lambda_{\mathrm{R}}^{2} \tau^{2}+\lambda_{\mathrm{R}} \tau_{\mathrm{r}}
$$

(2) a dependent (resulting from random shocks) failure contribution,

$$
\mathrm{q}_{C}=(1 / 2) \lambda_{C} \tau+\lambda_{C} \tau_{r}
$$

(3) a demand failure contribution,

$$
q_{D}=Q_{0}\left[Q_{1}+\gamma_{0}+\lambda_{R} \tau+\left(2 \tau_{r} / \tau\right)\right] ; \text { and }
$$

(4) a test and maintenance contribution,

$$
\mathrm{q}_{\mathrm{M}}=\gamma_{\mathrm{o}}\left[\gamma_{1}+\left(1-\gamma_{1}\right) \mathrm{Q}_{\mathrm{o}}+\left(2-\gamma_{1}\right)\left(\lambda_{\mathrm{R}}+\lambda_{\mathrm{C}}\right)(\tau / 2)+\left(2 \tau_{\mathrm{r}} / \tau\right)\right]
$$

where

$\lambda_{c}=$ occurrence rate of "shocks" that fail both components (common-cause failures),

$\mathrm{Q}_{1}=$ conditional probability of second component failure given that the first one fails, and

$\gamma_{1}=$ conditional probability of second component failure given that the first one fails because of a test or maintenance error. example,

Some terms in this expression of system unavailability can be interpreted readily. For

$(1 / 3) \lambda_{R}{ }^{2} \tau^{2}=$ contribution of random independent failures,

$\gamma_{0} \gamma_{1}=$ contribution of (dependent) maintenance errors on both components,

$\mathrm{Q}_{0} \mathrm{Q}_{1}=$ both components fail on demand, and

$\gamma_{0} Q_{2}=$ one component down because of human error and the other fails on demand.

Four major assumptions are made in this model.

(1) The systems under investigation are at standby mode. They are tested and, if necessary, repaired at periodic intervals.

(2) Each maintenance interval is independent of the others, and components are as good as new after test and maintenance. 
(3) The distribution functions of component failures are assumed to be exponential distribution with small failure rate.

(4) For one-out-or-two system, the above expressions are based on sequential testing. In the same paper, Apostolakis and Chu (1980) also derived the analytical expressions for the case of staggered testing.

\subsubsection{Correlation Among Statistical Parameters}

When uncertainty in the basic failure rates is taken into account, one should treat the correlation between the states of knowledge of elemental failure rates properly lest some substantial errors result from improper procedures. Some significant errors resulting from the mistreatment of correlation are presented in Apostolakis and Kaplan (1981).

For example, a system fails when one of two components of the same type in series fails. Two approaches can be used to calculated the system unavailability, $Q$ :

(1) $Q=q_{1}+q_{2}$ or

(2) $Q=2 q$,

where $q_{1}$ and $q_{2}$ are independent variables having the same distributions. Similarly, for two events in parallel, the two corresponding approaches would be

(1) $Q=q_{1} q_{2}$ or

(2) $Q=q^{2}$.

In the first approach, the knowledge of the analysts regarding the two identical components is treated as though they are independent quantities; therefore, the correlation of the state-ofknowledge uncertainty is ignored completely. However, the second approach is the correct one to use when the components are identical. It has been shown in Apostolakis and Kaplan (1981) that, for a series configuration, applying the first approach will not affect the mean but will underestimate the variance. However, for a parallel configuration, both mean and variance will be underestimated significantly.

\subsection{Accident-Sequence Quantification}

Accident-sequence quantification involves combining the initiating-event frequencies and basicevent probabilities into accident-sequence frequencies. The issues of rare-event assumption and parametric correlation for sequence quantification remain the same as for the system quantification and will not be repeated. However, logical approaches used in accident-sequence quantification and postaccident human errors will be discussed in detail.

\subsubsection{Logical Approaches for Accident-Sequence Quantification}

Two approaches of accident-sequence quantification are used in reactor PRAs (Rasmuson, 1991): large-event-tree/small-event-tree (LET) and small-fault-tree/large-fault-tree (SET) approach.

(1) The LET Approach. In the LET approach, the dependencies between the front-line systems and the support systems are modeled explicitly in the boundary conditions of the event trees. The fault trees are relatively small because they do not have to model the status of the support systems. Because each fault tree is developed for the front-line system under a 
specific set of boundary conditions depending on the states of the support systems, this approach also is called "event tree with boundary conditions." The LET requires a great deal of plant knowledge to construct the event trees.

(2) The SET Approach. In the SET approach, the fault trees defined under a given event tree are not assumed to be independent. An accident sequence is quantified by combining all fault tree minimal cut sets and therefore, this approach is sometimes called the "faulttree linking" approach. Fault-tree linking may lead to a very large fault tree containing.

\subsubsection{Post-Accident Human Error}

In this section, we discuss models and assumptions for post-accident human errors that involve procedure-oriented and scenario-specific activities that are integral parts of plant responses to initiating events. For each specific plant state, the operators must accomplish well-defined tasks for manual initiation, control, and alignment of plant emergency equipment or backup systems. These tasks generally are guided by the plant emergency-response procedures. The available time frame for successful response, the type of action that must be taken, and other factors that influence operator stress and confusion are the determining elements in the human reliability models.

Numerous models have been developed or proposed to address the three types of human actions mentioned above. Some models emphasize the time-reliability correlations, some apply expertopinion assessment techniques, and some others use data obtained from operator training on plant simulators. Apostolakis et al. (1988) give a detailed review on these models. In this section, only models that are applied to published PRAs are discussed.

\section{NRC Handbook of Human Reliability Analysis}

In the NRC human reliability handbook (Swain and Guttmann, 1982), the human error rate, $r$, is treated as a function of the available time, $T_{A}$, which is assumed to be known. Presumably, the handbook authors have estimated a stochastic distribution of $T$ (the time required by the crew to diagnose the plant situation accurately and act on it correctly) and the fraction of time that $T$ exceeds $T_{A}$. The state-of-knowledge uncertainty regarding $r$ is represented by a family of curves (upper bound, median, and lower bound) in the handbook, which are based on judgment.

\section{Operator Action Tree (OAT) and Human Cognitive-Reliability (HCR) Correlation}

Both OAT (Wreathall, 1981) and HCR (Hannaman et al., 1986) use time-reliability correlation curve(s) to quantify nonresponsive probabilities. The OAT method models the likelihood of the operators' success in diagnosing and implementing necessary safety functions after an initiating event. To conduct an OAT, the analyst first identifies the safety actions required for a given plant condition. The human error rates of these actions are then quantified by applying a time-reliability correlation (TRC).

The following assumptions are made in the OAT model.

(1) Most human errors are associated with cognitive behaviors; that is, they are most likely to occur during the diagnosis phase, which becomes the only stage considered in the quantification.

(2) It is assumed that the median response time is the determinant factor in the success probabilities of dynamic actions. All other factors are considered to be of secondary order. This human error probabilities can be considered a function of response time. 
(3) A TRC curve can be derived for each plant, and dynamic human-error probabilities are obtained from this correlation curve.

The HCR model differs from OAT in that, instead of a single time-reliability curve, three TRC curves that correspond to different modes of cognitive processes (knowledge-, rule-, and skill-based) are used in the quantification of nonresponsive probabilities. In addition to the assumptions made in the OAT model, the HCR model further assumes that each operator action can be classified clearly into one of the three cognitive processes.

\section{Success Likelihood Index Method/Multi-Attribute Utility Decomposition (SLIM/MAUD)}

Some PRAs quantify the event-level dynamic operator-error probabilities in the plant response model by an application of the success-likelihood index method/multi-attribute utility decomposition (SLIM/MAUD) (Embrey, 1985; Embrey et al., 1984), which is based on the assumption that human error rates under a particular situation depend on the combined effects of a relatively small set of PSFs that influence the operators' ability to perform the action successfully. For example, in the Beaver Valley PRA, seven PSFs have been selected to describe the range of problems that the operators face.

- Plant interface and indications of conditions

- Significant preceding and concurrent actions

- Task complexity

- Procedural guidance

- Training and experience

- Adequacy of time to accomplish action

- Stress

Based on the operators' response, the human error rate is evaluated by ranking the PSFs against two criteria: (1) their weight, which is the relative importance of the effect of each PSF on the likelihood of the success of the action, and (2) their rating, which tells how the operators and the actions are rated with respect to the PSFs.

The following are the major assumptions of the SLIM/MAUD approach.

(1) In the SLIM/MAUD approach, the available time, $T_{A}$, is no longer the only primary variable in the analysis. Instead, the human error rates are evaluated by the weights and ratings of a set of PSFs.

(2) The renormalization is a major problem in the approach.

(3) The validity of the "anchor" point is a major assumption in the approach.

\subsection{Statistical Models}

The rarity of accidents at nuclear power plants has challenged the relative-frequency-based interpretation of probability to which most engineers and physicists are accustomed. The lack of data has made subjective judgment an inseparable part of a PRA. Although too broad a subject for this review, this section briefly covers some of the very fundamental statistical issues (e.g., the 
interpretation of probability, the assignment of prior probability, the use of evidence) that have been the subjects of debate over the years.

\subsubsection{Subjective vs Relative-Frequency Interpretation of Probability}

The interpretation of probability is not just an issue of philosophical debate because the acceptance of one interpretation over another determines which statistical models to use in estimating parameters and hypothesis testing.

\subsubsection{Relative-Frequency Interpretation of Probability}

Probability is defined in the relative-frequency framework in the following sense (von Mises, 1939; Reichenbach, 1949). If an event $A$ occurs $m$ times in $n$ observation, the relative frequency of event $A$ is defined as $m / n$. The probability of event $A$ then is defined as

$$
p(A)=\lim _{n \rightarrow \infty} \frac{m}{n} .
$$
following.

The underlying assumptions in the relative-frequency interpretation of probability include the

(1) The fundamental axiom of this approach is that the limit of the relative frequency exists. For one who exercises the relative-frequency interpretation of probability, only probabilities defined within a repeated observations can be evaluated, and only these probabilities have any scientific interest. No attempt is made to assign a probability to a single event.

(2) The definition of probability in this framework does not allow for any subjective knowledge of the analyst to be used beyond direct observations.

\subsubsection{Subjective Theory of Probability}

Ramsey (1931) and de Finetti (1974) proposed that probability should be interpreted as a measure of degree of belief. Savage (1954) further proved that if one follows a specific pattern of choice [e.g., if one thinks that event $A$ is more likely than event $B$, then $P(A) \geq P(B)$ ], his degree of belief would obey the calculus of probability. De Finetti (1974) made the important connection between the probability of the subjective world and the relative frequency of the objective world through the concept of exchangeability and the theorem of representation, in which the classical concept of random processes is placed into the framework of subjective probability.

In the field of PRAs, most PRA analysts have either taken the subjective probability point of view or incorporated models based on the theory of subjective probability theory into their work. Papers written on the subject include Apostolakis (1978), Kaplan (Kaplan and Garrick, 1981), and Apostolakis (1990).

The three fundamental concepts of the theory of subjective probability are degree of belief, coherence, and exchangeable events. The principal assumption is that the subjective probability is the measure of the degree of belief. Also, it is always possible to elicit a unique degree of belief on the same event under the same circumstances from a coherent individual. 


\subsubsection{Bayesian vs Classical Statistics}

Bayesian statistics is derived from the subjective interpretation of probability, whereas classical statistics follows the relative-frequency interpretation of probability.

\subsubsection{Classical Statistics}

Point Estimates of Parameters. The point estimate of a parameter in classical statistics is a statistic obtained from computations on, and only on, the observed values of the random variable that serves as an approximation to the parameter. Some commonly used point estimates of parameters are the method of moments and the maximum-likelihood estimate (Hoel, 1971). Unbiased and minimum variance are among some of the preferred properties of the point estimates.

Because classical point estimates only account for the observed value of the random variable, sometimes the result can be useless when events are rare. For example, the maximum likelihood estimate (MLE) gives the point estimate of $r / n$ for the parameter, $p$, in a binomial distribution, where $r$ is the number of events observed in $n$ observations. In the case of component failures, if no failure was observed, the MLE of $\mathrm{p}$ would be 0 , a statement too strong to be accepted by most. Some PRA analysts choose to "fix" this undesired situation by taking the point estimate as

$$
\mathrm{p}= \begin{cases}\mathrm{r} / \mathrm{n}, & \mathrm{r} \neq 0 \\ (\mathrm{r}+0.33) / \mathrm{n}, & \mathrm{r}=0\end{cases}
$$

This corresponds roughly to a confidence limit of about $28 \%$ (Georgin and Signoret, 1981).

Confidence Interval. Let $\lambda$ be the unknown parameter for a probability distribution of a random variable, $T$ (for instance, $\lambda$ could be the failure rate of a component and $T=t$ the time to failure). The 1 $\alpha$ upper confidence limit for $\lambda, U_{\alpha}$, is defined as a statistic $U_{\alpha}=f U(\alpha, t)$, such that for all possible values of $\lambda$,

$$
\mathrm{p}\left(\mathrm{U}_{\alpha} \geq \lambda \mid \lambda\right)=1-\alpha .
$$

Similarly, for the $\alpha$ lower confidence limit, $L_{\alpha}$, for $\lambda$ is defined as another statistic, $L_{\alpha}=f_{L}(\alpha, t)$, such that for all possible values of $\lambda$,

$$
\mathrm{p}\left(\lambda \leq \mathrm{L}_{\alpha} \mid \lambda\right)=\alpha \text {. }
$$

Note that in classical statistics, the confidence limits, $U_{\alpha}$ and $L_{\alpha}$, are random variables but the parameter, $\lambda$, is not. Because it is generally not known what $\lambda$ is, the interpretation of the upper bound is that if we assert that $\lambda \leq U_{\alpha}$ for each sample on which the statistic $U_{\alpha}$ is calculated, then in the long run, there is a proportion, $1-\alpha$, of the samples where that would be the case. Confidence intervals are almost impossible to propagate through fault trees and are rarely, if ever, used in PRAs.

\subsubsection{Bayesian Statistics}

The Bayesian approach that has been used often in PRA data analysis is based on the subjective interpretation of probability. The data-updating technique in the Bayesian approach is through Bayes's theorem. For a discrete random variable $\lambda$, Bayes's theorem takes the form (Apostolakis et al., 1980) 


$$
p\left(\lambda_{1} \mid E\right)=\frac{p\left(\lambda_{i}\right) L\left(E \mid \lambda_{i}\right)}{\sum_{i} p\left(\lambda_{i}\right) L\left(E \mid \lambda_{i}\right)},
$$

where

$$
\begin{aligned}
\mathrm{p}\left(\lambda_{\mathrm{i}} \mid \mathrm{E}\right) & =\text { the posterior probability distribution of } \lambda \text {, given evidence } E ; \\
\mathrm{p}\left(\lambda_{\mathrm{i}}\right) & =\text { the prior probability distribution of } \lambda \text { before observing evidence } E ; \text { and } \\
\mathrm{L}\left(\mathrm{E} \mid \lambda_{\mathrm{i}}\right)= & \text { the likelihood function that evidence } E \text { would be observed if } \lambda \text { takes the value } \\
& \lambda_{\mathrm{i}} .
\end{aligned}
$$

For a continuous $\lambda$, we write Bayes's theorem in the form

$$
f(\lambda \mid E)=\frac{f(\lambda) L(E \mid \lambda)}{\int_{0}^{\infty} f(\lambda) L(E \mid \lambda) d \lambda},
$$

where

$f(\lambda \mid E)=$ the posterior probability density function of $\lambda$, given evidence $E$,

$f(\lambda)=$ the prior probability density function of $\lambda$ before observing evidence $E$, and

$L(E \mid \lambda)=$ the likelihood function that evidence would be observed given $\lambda$.

The basic assumptions of Bayesian approach to data analysis include the following.

(1) The "state of knowledge" of the analysts is presented in the form of a probability distribution over the range of possible values of that quantity.

(2) The probability associated with a particular numerical value of an uncertain but measurable quantity indicates the likelihood that this numerical value is the correct one.

The fact that parameters, such as failure rates, are allowed to be distributed has been found very useful by PRA analysts. Furthermore, it is relatively straightforward to propagate distributions through fault trees (unlike confidence intervals).

Bayesian techniques are often used in the PRA data analysis. Generally, three types of information are involved.

$E_{1}$ : General engineering knowledge of the design and the manufacture of the equipment in question and the frequency to be expected on that basis.

$E_{2}$ : The historical performance in other plants similar to the one in question.

$E_{3}$ : The past experience in the specific plant being studied. 
The information of types $E_{1}$ and $E_{2}$ together constitutes the "generic" information; $E_{3}$ is the "plant-specific" information. Thus, the objective of data analysis is to develop a prior distribution based on the generic information and then use Bayes' theorem and plant-specific information to specialize the generic distributions.

\subsubsection{The Assignment of Prior Distribution}

Two assumptions generally are made regarding prior distributions, $f(\lambda)$ 's, and evidence, $E$.

(1) Many PRAs based the prior distributions on generic information from published documents that contain information representing industrial collective experience [for example, the Reactor Safety Study (USNRC, 1975), IEEE-500 (IEEE, 1977), and NUREG/CR-1363 (Hubble, 1980)].

(2) The lognormal distribution is used extensively to represent the analysts' state of knowledge. The lognormal distribution has the underlying assumption that the random variable under consideration is restricted to positive values (i.e., 0 to $\infty$ ).

\subsubsection{Reactor Safety Study Approach}

The Reactor Safety Study (USNRC, 1975) gives, for each failure rate, not a single number but a range of numbers. For example, for the failure rate, $\lambda$, of pumps under normal environments, the range is $3 \times 10^{-6}$ to $3 \times 10^{-4}$ failures per hour, which are described in the Reactor Safety Study as the 5th and 95th percentiles of a lognormal distribution. These distributions are interpreted as "population variability curves" or curves showing the variation of performance of individual components within the population. They are frequency distributions representing the variability stemming from different manufacturers, different models, and different operating and maintenance conditions. If all one knows about a specific component is that it is one member of the population, then one would set the prior probability curves that express one's state of knowledge equal to the variability curve.

The lognormal distributions of parameters in the Reactor Safety Study are based on point estimates of failure rates supplied by experts (organization, individuals, etc.). The 5th and 95th percentiles are the end points of the "assessed range." The decision to use the end points of the range as the 5th and 95th percentiles reflects the belief of the Reactor Safety Study team that there is a 0.90 probability that the failure rates of various components in US nuclear plants are to be found with this assessed range. However, the results of Apostolakis et al. (1980) suggest that perhaps the probability of 0.90 is too high. In some PRAs, it was decided to broaden the generic curves by taking the endpoints of the assessed range as the 20th and 80th percentiles of a lognormal distribution.

\subsubsection{The Use of IEEE Std-500 Data}

IEEE Std-500 contains data for electronic, electrical, and sensing components. The reported values were mainly synthesized from the opinions of some 200 experts (a form of the Delphi procedure was used). Each expert reported a "low," "recommended," and "high" value of the failure rate under normal conditions and a "maximum" value that would be applicable under all conditions (including abnormal ones). The estimates were pooled using geometric averaging techniques, e.g.,

$$
\lambda_{\max }=\left[\prod_{\mathrm{i}=1}^{\mathrm{n}} \lambda_{\max , \mathrm{i}}\right]^{1 / \mathrm{n}} .
$$


The standard does not recommend a distribution but suggests that the recommended value be used as a "best" estimate. Apostolakis (1982) suggests using the best estimate to be the median value and using the maximum value as the 80 th percentile of the lognormal distribution.

\subsubsection{Two-Stage Bayesian}

Kaplan (1983) proposes a "two-stage" procedure by which the generic distribution is derived using the evidence $E_{2}$ explicitly in Bayes's theorem. The lognormal curve that expresses plant-to-plant variability is embedded in a space of functions, which consists of all lognormal curves. Starting with a uniform prior probability distribution, the evidence $E_{2}$ is used in Bayes' theorem to produce a new family of lognormal curves each having a posterior probability of being the true curve. The average of these curves is then the generic distribution that replaces the Reactor Safety Study or IEEE-500 curves.

\subsection{External Events Analysis-Seismic Risk Assessment}

There are four main parts to the seismic risk assessment (Cornell, 1968).

(1) The seismic hazard assessment is used to estimate the frequency of occurrence and magnitude of ground-motion acceleration induced at the plant site by earthquakes.

(2) The seismic fragility analysis used is to estimate the response of essential structures and system components for which failures may significantly influence the likelihood of core melt.

(3) In the system modeling, seismic event trees and fault trees are developed for each seismicinduced accident sequence.

(4) The quantification analysis is used to estimate the plant risk of seismic-induced events.

\subsubsection{Seismic Hazard Assessment}

The estimation of the frequency of the occurrence and magnitude of ground-motion acceleration starts with a detailed review of the earthquake records reported for regions near the site. Based on the location and intensity of observed earthquakes and on geological and tectonic data, these regions are divided into several seismogenic zones. Within each zone, it is assumed that there is an equal likelihood of an earthquake to occur, and the frequency of earthquakes at various magnitudes is estimated based on historical records of the specific zone. Different attenuation models then are used to postulate the ground-motion acceleration at the plant site that is induced by an earthquake of a given magnitude occurring at a certain distance. There are some significant discrepancies among the assumptions and thus in the results obtained from different seismogenic models and attenuation models. Based on the weights assigned by experts to these models, the results are aggregated into one frequencyacceleration curve, which is used in the seismic risk assessment to represent the ground-motion hazard at the plant site.

The seismic hazard in PRAs is evaluated for several (often six) measures for earthquake ground motion, peak ground acceleration (PGA), and pseudo-relative velocity-response spectra (PSRV) at several frequencies (e.g., 1, 2.5, 10, and $25 \mathrm{~Hz}$ ). The hazard results for these ground-motion measures are used to define the response spectrum input to the fragility analysis. Furthermore, the seismic hazard is characterized in terms of spectral acceleration, $S_{a}$, at a specific frequency (e.g., $5.0 \mathrm{~Hz}$ ). 


\subsubsection{Seismic Fragility Analysis}

The seismic fragility analysis estimates the margin of failure of components and structures as compared with their design-basis loads, which typically is defined at one-half of the safe-shutdown earthquake (SSE) load. The original design analysis, if available, is used as the starting point in most seismic PRAs. The fragility analysis consists of systematically evaluating the conservatism (and nonconservatism) embedded in the analysis to develop the median capacities. Based on variabilities in the basic underlying variables (e.g., equipment response, equipment capacity, and structure response parameters), the statistical description of each component capacity is determined, which defines the shape and spread of the fragility curves. The following are some general assumptions that are often made when conducting a fragility analysis.

(1) The objective of a seismic PRA is to produce results that are realistic (i.e., neither too conservative nor too optimistic). However, in the fragility analysis of some PRAs conservative assumptions are made for components that do not influence the final result in order to speed up the analysis. A more realistic analysis is performed only when it is determined that a component is a significant contributor. This is to ensure that resources are directed toward the components that are the most important with regard to seismicrelated plant safety.

(2) The analysts also made conservative judgments when the information available about a component is not detailed enough. For example, certain anchorage details cannot be determined in many cases. Conservative assumptions then are made as long as they can be well defended. Under this strategy, some of the fragility values are, in general, conservative.

(3) The analysis often is conducted based on a specific spectrum and on forces for the basic structure-response input, which is, in turn, based on the floor response spectra and on forces in the structural elements calculated from the soil-structure interaction (SSI) analysis.

(4) A plant-specific analysis is conducted for equipment such as pumps, tanks, and heat exchangers, whereas a generic analysis is conducted for piping, valves, relay chatter, and cable trays. Different fragility curves are developed for different piping conditions and general arrangements. For underground piping, a wave passage analysis and a differential movement analysis (because of ground settlement) often are conducted for the critical pipe configurations; the analyses include the effects of water temperature and potential brittle failure because of high nil-ductility temperature values. Above-ground piping is defined as being hot or cold (i.e., potential nil-ductility failure), previously analyzed or unanalyzed, and secured with typical anchor bolts or with lead expansion anchors.

(5) The definitions of the scope of the fragility analysis for structures and for equipment are closely tied to the plant operations and to the use of the fragility data in the plant systems model. For example, the motor-control-center (MCC) relays for the rotovalves must remain open during the earthquake; therefore, relay chatter during a seismic event must be considered. However, complete failure of the MCC, which serves the connecting cables, is not a safety concern because any signal to close the valves would be aborted.

(6) Fragility analyses of structures and equipment are based mainly on the stress analyses conducted in the design period. If available, data obtained from latter structural and stress analyses are included in the fragility analysis.

(7) For elements of structures that support safety-related equipment, failure is assumed to occur when inelastic deformations because of seismic motions are large enough to 
potentially affect the operability of equipment that is attached. This is a conservative definition of failure of a structure and is at a lower level than total collapse of an element or building. Considerable margin exists for structural collapse compared with the capacities reported in the fragility analysis for failures related to equipment functionality.

(8) Safety-related equipment is assumed to fail when it can no longer perform its function. Equipment failures can be caused by direct inertial loads that are seismically induced, relative displacement-induced loads, or the failures of an adjacent structure or component that falls onto and fails the safety-related component. For the case of direct seismically induced failure of equipment, fragility curves are based on one of the following three types of failures: loss of function, rupture of a pressure boundary, or loss of anchorage.

\section{Loss of function}

- Seizure of a motor shaft as a result of bearing damage

- Binding of a valve because of distortion of its operator

- Excessive deflection of a fan blade (causing it to rub against the fan housing and subsequently to bind or to reduce the flow capacity)

- Relay chatter leading to breaker trip or malfunction of a valve

- Cutting or severing of electrical cables

- Gross failure of air ducting

Rupture of a pressure boundary

- Rupture of a pipe or valve casing

- Buckling of a tank causing failure of the tank wall

- Rupture of a valve casing because of impact

Anchorage failures

- Anchor bolt failure resulting from either rupture of the bolt itself or cracking and pullout from the concrete support

- Weld failure

- Buckling or excessive yielding of a leg that is part of the component support system

- Shear pin failure

(9) Examples of indirect failures caused by adjacent structures or components include

- failure of a block wall onto batteries or adjacent tank;

- failure of block, tile, or transit walls that support safety-related equipment; 
- failure of ducting over the central control room (CCR) that could fall and impact the plant operators; and

- failure of the portion of building that supports the stack can generate a missile, penetrate the manhole, and strike pipe or equipment.

One fragility model that has received some recognition in seismic fragility analysis is that of treating the seismic capacity, $A$ (expressed in the same unit used for ground motion acceleration), of a component in response to a failure mode as a random variable (Kennedy et al., 1980). It is further assumed that the uncertainty of the fragility basically comes from two sources: the randomness of the component capacity, $\varepsilon_{R}$, and the lack of knowledge of the analysts, $\varepsilon_{U}$, regarding the physical modeling of structure and material. The most frequent form for the expression $A$ is

$$
A=\hat{A} \varepsilon_{\mathrm{R}} \varepsilon_{\mathrm{U}}
$$

where $\hat{A}$ is the median value of the seismic capacity of the component. Most.fragility analyses further assume that both $\varepsilon_{R}$ and $\varepsilon_{U}$ are lognormally distributed random variables.

The median capacity, $\hat{A}$ (defined at the ground surface level in the free field), often is reported in terms of the $5 \%$-damped spectral acceleration value at the $5.0-\mathrm{Hz}$ frequency. This capacity parameter is defined as the average of the two orthogonal, horizontal-ground spectral acceleration hazard curves. By using this definition in the fragility analysis, there is a consistent interface between the hazard and the fragility analysis.

Randomness in the seismic fragility corresponds to the inherent (or irreducible) variability associated primarily with the component response to the earthquake ground motion. The randomness of the ground motion results from the peaks and valleys in the ground response spectrum and earthquake duration. The random phasing of the response of structure and equipment dynamic modes and of the three orthogonal earthquake components also contributes to randomness.

Uncertainty that results from lack of knowledge is that part of the variability associated with model uncertainties and limited data. Uncertainty of this kind is associated primarily with the lack of knowledge of the capacity model and the properties of the structural materials. This variability can be reduced in principal by additional testing or analysis and more understanding of the physical models.

The fragility of a component thus obtained is the probability of component failure, conditioned on a given value of the ground motion parameter (e.g., ground-level spectral acceleration). The fragility of each structure and equipment item ultimately is expressed in terms of the double lognormal model as defined by the following three parameters.

(1) Median, ground-spectral acceleration capacity (in terms, for example, of 5\%-damped spectral acceleration evaluated at $5.0 \mathrm{~Hz}), \hat{A}$

(2) Logarithmic standard deviation for capacity because of randomness, $\beta_{R}$

(3) Logarithmic standard deviation for capacity because of lack of knowledge, $\beta_{2}$ 


\subsubsection{System Modeling}

Seismic event and fault trees are developed based on the information obtained from internal event trees and fault trees. System event and fault trees often are quantified using Monte-Carlo simulation techniques based on the following assumptions.

(1) In most seismic PRAs, identical components that are installed at the same elevation and orientation in the plant are assumed to fail together as a result of the same seismic challenge. In other words, the seismic quantification assumes that total dependence exists between seismic failures of identical components. The model does not allow for partial dependencies between identical components (i.e., one component survives after an earthquake and another identical component fails).

(2) It generally is assumed that electrical power cannot be recovered during or immediately after a seismic event.

(3) For operator actions during and after an earthquake, it is assumed that the reactor operating crew is called on to perform several functions to bring the reactor to a safe state after the occurrence of a damaging earthquake. The possibility of failure to perform these actions has been derived in a manner that accounts for the size of the crew, the time available for corrective action, the location in the plant for the required action, the size of the earthquake, and the previous analysis of operator error in an internal events analysis.

(4) In analyzing the response of the reactor to seismic events, many assumptions are made to reduce the quantification time and to simplify the analysis. For example, in the Savannah River seismic PRAs, it is assumed that the actuation of the emergency core cooling systems eventually leads to core damage if no water is being added to the coolingwater basin. Also, in the same analysis, piping is assumed to rupture in a double-ended guillotine fashion and to spill water into the building. No recovery is modeled because all breaks of pipes larger than 10 in. in diameter will overcome sump capacity with no operator action. It is further assumed that emergency electrical power is always required to pump spilled water from the reactor building. 


\section{AGING RISK ANALYSES AND PHYSICAL MODELS}

\subsection{Introduction}

The models and methods used to estimate the reliability of components and systems subject to age-related degradation are reviewed in this section. The primary intent of this work is to review the "linearly increasing failure rate model" proposed in Vesely (1987), as well as the "aging effect on plant risk model" proposed in Vesely et al. (1990). Also included is a review of aging effects on maintenance The principal question that is investigated is under what conditions a probabilistic analysis based on physical principles leads to a failure rate that can be approximated reasonably by a linearly increasing failure rate. To answer this question, several models that have been proposed for the three dominant degradation mechanisms (fatigue, irradiation embrittlement, and corrosion) are examined. The following topics are presented for each model.

(1) The objectives of the model. This involves describing the situations for which the model applies and is intended to be used.

(2) The formulation of the model. This involves presenting the pertinent equations and describing how the model is used.

(3) The assumptions made in formulating the model.

(4) A commentary on whether the assumptions described are reasonable and, if not, for which situations they are reasonable.

The Taylor expansion approach to the estimation of the effect of aging on plant risk, is briefly described and discussed in Sec. 2.2. In Sec. 2.3, the aging effects on maintenance are discussed. In Sec. 2.4 , various component reliability distribution models are described and discussed, including the linear aging reliability model. In Sec. 2.5, physically based methods and models for estimating component reliability are described. The first part of Sec. 2.5 contains a description of the important potential aging mechanisms that either have been observed or are thought to be important for nuclear power plant components and structures. The remaining parts of Sec. 2.5 describe reliability models related to three important degradation mechanisms: fatigue, irradiation embrittlement, and stress-corrosion cracking. For each of these mechanisms, an attempt is made to relate the physical fundamentals to the linear aging reliability model and to determine what conditions, if any, under which the linear aging model would be adequate to describe the time-dependent failure probability because of those mechanisms.

\subsection{Taylor Expansion Approach for Incorporating Aging Effects into PRAs}

\subsubsection{The Model's Objectives}

The Taylor expansion approach presented in Vesely et al. (1990) is a methodology developed to incorporate aging effects into a PRA to estimate the effect of aging on plant risk. The authors suggest that component aging and risk models should be analyzed separately; aging models are introduced at the component level, and risk analysis is performed at the system and plant level. This approach relates the change in individual component unavailabilities because of aging to the change in the overall plant risk. The results of the analysis then can be used to evaluate the effectiveness of maintenance and surveillance in controlling aging and to direct resources to those SSCs that are most important to nuclear power plant risk. 


\subsubsection{The Model's Formulation}

Let $\mathrm{R}$ be an appropriate risk measure (for example, the core-damage frequency), which can be expressed in terms of component unavailabilities as

$$
R=\sum_{i} R_{i} q_{i}+\sum_{i>j} R_{i j} q_{i} q_{j}+\sum_{i>j>k} R_{i j k} q_{i_{j k}}+\ldots+R_{12 . . n} q_{1} q_{2} \ldots q_{n}
$$

where $R_{i}, R_{i j}, \ldots$ are coefficients, and the $q_{i}$ 's are the component unavailabilities. Given the above form, the Taylor expansion of the risk increment can be written as

$$
\Delta R=\sum_{i} S_{1} \Delta q_{i}+\sum_{i>j} S_{i j} \Delta q_{i} \Delta q_{j}+\sum_{i>j>k} S_{i j k} \Delta q_{i} \Delta q_{j} \Delta q_{k}+\ldots+S_{12 . . n} \Delta q_{1} \Delta q_{2} \ldots \Delta q_{n},
$$

where $S_{i}, S_{i j}, \ldots$, are sensitivity coefficients that are related to $R_{i}, R_{i j}, \ldots$, and the $\Delta q_{i}$ 's are the incremental changes in component unavailabilities. In this case, the change in component unavailability results from aging.

\subsubsection{Assumptions}

In this section, we discuss the major assumptions made when the Taylor expansion approach is used to incorporate aging effects into PRAs. In this review, we have benefited from a discussion of these assumptions in a memorandum by Lois (1992).

The Taylor series expansion basically states that if $f\left(x_{1}, x_{2}, \ldots, x_{n}\right)$ is a function of a set of independent variables, $x_{1}, x_{2}, \ldots, x_{n}$, then for finite incremental changes $\Delta x_{i}$ of $x_{i}$, we get the following equation:

$$
\Delta f\left(x_{i}, \ldots, x_{n}\right)=\sum_{i} \frac{\partial f}{\partial x_{i}} \Delta x_{i}+\sum_{i>j} \frac{\partial^{2} f}{\partial x_{i} \partial x_{j}} \Delta x_{i} \Delta x_{j}+\ldots
$$

The following assumptions are made in this expansion.

(1) The mathematical form for the function $f\left(x_{1}, x_{2}, \ldots, x_{n}\right)$ remains the same before and after the increments of $x_{j}$ 's. In the context of aging, this means that the structure of the risk assessment model remains unchanged after introducing the effects of aging. In other words, it is assumed that the risk analysts have already included in the plant risk model all component, system and structural failure modes that are related to the aging processes.

(2) The effect of aging on plant risk, $\Delta R$, is through the changes in component unavailabilities, $\Delta q$, only.

\subsubsection{Commentary}

(1) Aging may enhance the importance of terms that have been judged to be negligible (or have not been included) in the basic PRA model. For example, consider the failure probabilities of passive components such as the pressure vessel and piping. In most PRAs, the failure probabilities of passive components are considered to be negligible and are not modeled when quantifying system unavailabilities. However, failures in the primary 
system piping are usually considered as initiating events. Even though the proposed model deals exclusively with component unavailabilities, it would not be difficult to extend it to include initiating-event frequencies. If the failure probabilities of major passive components increase significantly because of aging, the effect on overall plant risk will be missed unless those components are included in the original risk model.

(2) Even though the model is presented in terms of changes in component unavailabilities, the only contribution to the unavailabilities currently considered is that resulting from changes in the failure rates. In fact, explicitly expressing the model in terms of failure rates, instead of component unavailabilities, would be more appropriate because it would avoid problems arising from the same parameter appearing in two or more contributors to system unavailability. For example, for a one-out-of-two standby system of two nominally identical components with standby failure rate $\lambda$, which are tested every $\tau$ hours for $\tau_{\Gamma}$ hours, the system unavailability is (Apostolakis and $\mathrm{Chu}, 1980$ )

$$
\mathrm{Q}=\frac{\lambda^{2} \tau^{2}}{3}+\lambda \tau_{\mathrm{r}}
$$

In this expression, the first term is the system average unavailability because of "random" failures of both components between tests, and the second term is the system average unavailability because of one component being disabled as a result of testing and the other failing during the test. It is impossible to identify unavailabilities associated with a single component in Eq. (2.4). If the changes because of aging are considered at the level of the failure rate, the proposed model still would apply.

(3) However, even with the above modification contributions to component unavailabilities other than changes in the failure rates are not considered. Other contributions that could be important include factors such as the increase in the maintenance contribution to the unavailability because of the increase in the frequency of repair and maintenance outages of aged components.

(4) Considering the effect of aging on risk through component unavailabilities is too restrictive because there are situations where terms other than component unavailabilities may contribute to risk. For example, aging components, e.g., batteries, may reduce the available time for operator action during accidents, thus altering the probability of successful operator actions. More generally, any contributions that are analyzed based on the physics of the situation cannot be placed in this form. This includes seismic and fire analyses and Level 2 phenomena.

(5) The restriction to component unavailabilities and failure rates masks the physics behind the various contributors to risk, thus inhibiting risk management. For example, in fatigue failures involving crack propagation, the probability of cracks of given sizes is important, and changing this probability affects the overall risk. However, this effect cannot be evaluated (or even considered) in the proposed formalism. This point is discussed in more detail later (see Fig. 2.1).

In another example, erosion-corrosion inspections at the Susquehanna Steam Electric Station identified an unexpectedly high rate of erosion-corrosion in certain main feedwater piping segments. Consideration of the physical mechanism of erosion-corrosion, combined with the information obtained through inspections, might allow better prediction of the rate of erosion-corrosion, and might point out critical locations where inspection resources should be concentrated. 
(a)

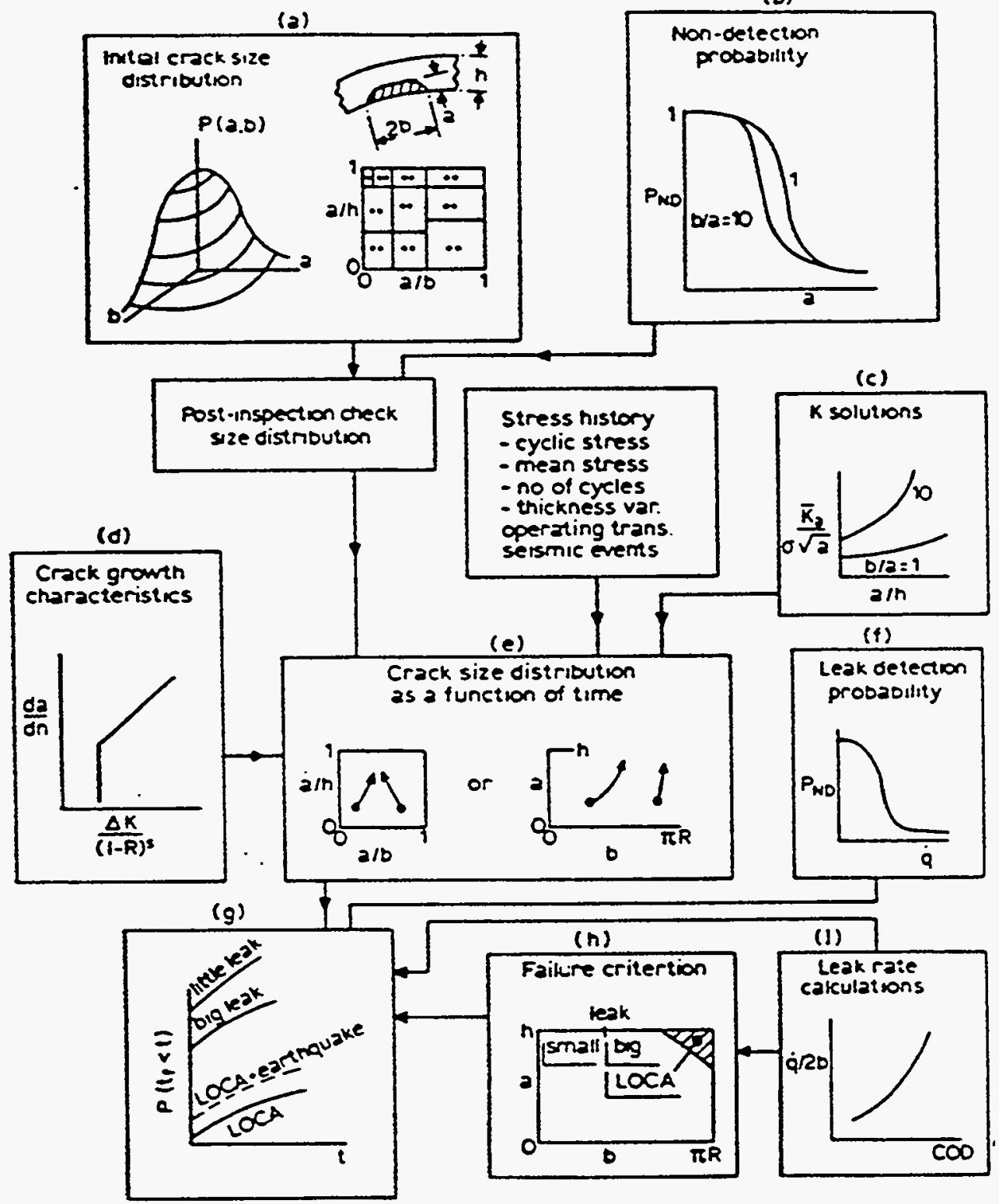

Figure 2.1 The PRAISE methodology (Harris et al., 1981) 
It should be noted that the idea of building on the existing PRA model to assess the effect of aging on plant risk is a good one and should be preserved as much as possible.

\subsection{Aging and Maintenance}

The potential effect of aging on maintenance practices is of some interest. Efforts to incorporate aging and degradation effects into maintenance effectiveness evaluations were carried out by Samanta et al., in NUREG/CR 5612 (Samanta et al., 1991). The report consists of several parts. The first part consists of the development of a theoretical model to combine component maintenance, degradation, and failure. This model development includes discussion of two items of interest: maintenance effectiveness and the incorporation of aging effects into degradation modeling. Only the definition of maintenance effectiveness is used in subsequent data analysis. The second part consists of performing statistical analysis on actual data to derive actual degradation and failure frequencies, which are required to estimate maintenance effectiveness.

\subsubsection{The Model's Use}

NUREG/CR-5612 describes "...a modeling approach to analyze component degradation and failure data to understand the aging process of components." Further, "The methodology presented also discusses the effectiveness of maintenance as applicable to aging evaluations." One potential use of the model is to estimate failure rates from degradation rates. The idea is that much more degradation data are available than failure data. Another potential use of the model is the determination of the effectiveness of various maintenance procedures carried out for a type of component.

\subsubsection{The Model's Assumptions} assumptions.

In developing their theoretical degradation model, Samanta et al. made the following

(1) Degradation always precedes failure; i.e., there are no catastrophic failures.

(2) When a component is repaired after a failure, the operational state reflects more restoration than when on-line maintenance is performed.

(3) When maintenance is performed following detection of a degraded condition, the component is restored to a maintained state that reflects less restoration than when repair is performed after a failure.

(4) Only repairable components are studied.

(5) Only one degraded state is considered.

(6) Only corrective maintenance is considered; i.e., maintenance is performed after a degraded condition is detected.

(7) Nondetected degradation is not treated by the model.

(8) Corrective maintenance is performed every time a degraded state is detected.

(9) No preventive maintenance is considered. 
(10) The repair time is zero; i.e., it takes no time to go from the failed state to the operational state.

(11) The time to go from a degraded state to a maintained state is zero; i.e., maintenance takes no time.

(12) Test frequency to detect component degradation is ignored.

(13) Aging begins at some threshold time, and both the failure frequency and degradation frequency increase from that time.

(14) The transition probability densities between states depend only on the time interval.

(15) The steady-state transition probability densities can be applied to aging dependent evaluation of failure and degradation frequencies.

(16) The steady-state transition probabilities developed from the steady-state case also apply to the aging case.

Figure 2 of the report, as drawn, showing the Markov state diagram is reproduced here as Fig. 2.2. This figure shows the underlying transitions and states used in developing the model. Section 3.2 of the report, which discusses transition probabilities, is reproduced here as Fig. 2.3.

\subsubsection{Discussion}

The assumptions and diagrams in this report are unclear. For example, the Markov model as shown in Fig. 2.2, when combined with the transition probabilities presented in Fig. 2.3, leads to an apparent conflict within the model. There appear to be inconsistencies in the presentation of the report.

Figure 2.2 shows no m-state to f-state transition, yet in Fig. 2.3 a $\mathrm{P}_{\mathrm{MF}}$ is defined. Furthermore, $P_{M F}$ is defined to be equal to $P_{D F}$, which would not in general be true. Also, in Fig. 2.3 it is stated that $P_{D M}=1$. This implies that $P D F=0$ as can be seen from the definition of Markov transition probabilities $\mathrm{P}_{\mathrm{ij}}$ (Billinton and Allan, 1983) for discrete states, namely the probability a system in state $\mathrm{i}$ will make its next transition to some different state $j$, summed over all $j$, is unity:

$$
\sum_{j, j \neq i} P_{i j}=1
$$

Since, Fig. 2.2 only shows two possible transitions from state $D$, namely $M$ and $F$, if it is assumed that one of these transitions occurs with certainty $\left(\mathrm{PDM}_{\mathrm{D}}=1\right)$, then the other can never occur $\left(\mathrm{P}_{\mathrm{DF}}=0\right)$.

Furthermore, $\mathrm{PDF}=0$ implies that the failed state can never be reached, which is contrary to parts of the problem description. This appears to correspond to perfect degradation detection and maintenance on the component. In other words, when the component's operating characteristic degraded beyond some allowable value, it would always be detected instantly and correctively maintained, putting it into the $\mathrm{m}$ state. From the $\mathrm{m}$ state, it would transition back to the $\mathrm{d}$ state to repeat the process, never reaching the $f$ state.

As presented in Figs. 2.2 and 2.3, the only method for obtaining a nonzero PDF is that PDM not be equal to 1 . Consistent with the description of the model, this would seem to imply that the component failed through an undetected degradation that did not receive corrective maintenance, apparently 


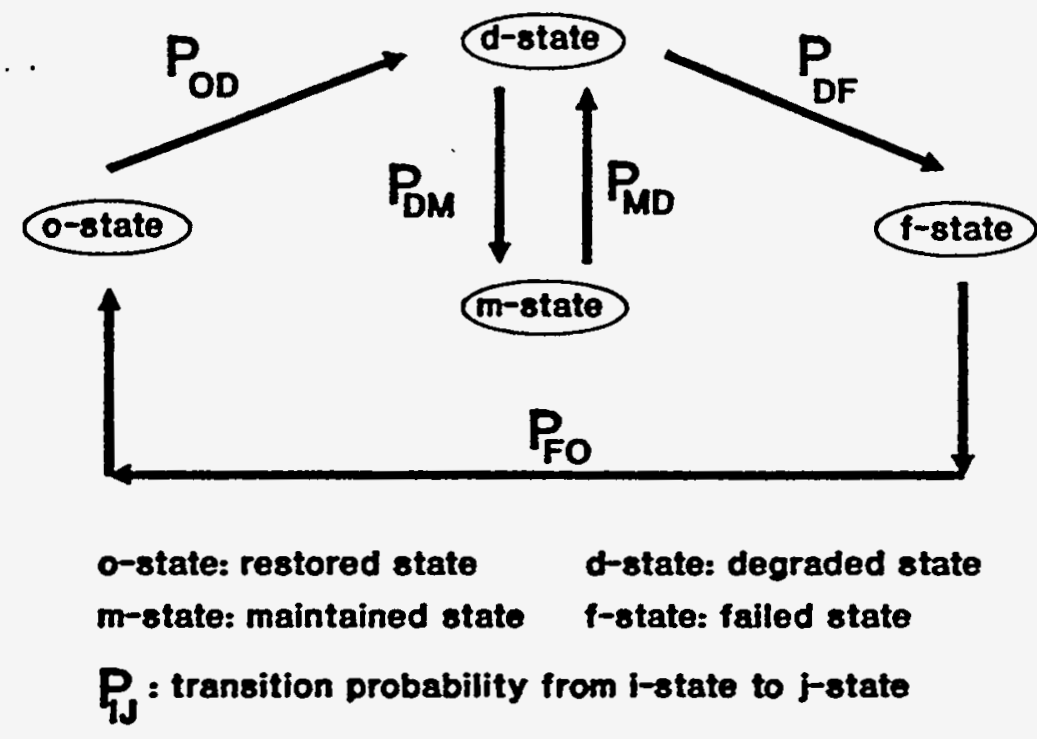

Figure 2.2 Markov state diagram (Samanta, 1991) 


\subsection{Transition Probabilities}

The transition probabilities among the various states are as follows:

$$
\begin{aligned}
P_{\mathrm{OD}}= & \text { probability that degradation occurs after the component is restored with } \\
& \text { no failure before a degradation } \\
= & 1 \text { since we assume degradation always precedes failure } \\
\mathbf{P}_{\mathrm{DM}}= & \text { probability that maintenance is carried out once a degraded state is } \\
\text { identified } & =1 \text { since maintenance will be performed to remedy the degraded state. } \\
\mathbf{P}_{\mathrm{MD}}= & \text { probability that degradation occurs after a maintenance before a failure } \\
& \text { occurs. } \\
\mathrm{P}_{\mathrm{DF}}=\mathrm{P}_{\mathrm{MF}}= & \begin{array}{l}
\text { probability that failure occurs after a maintenance (performed following } \\
\text { detection of a degraded state) with no intermediate degradation. }
\end{array} \\
\mathrm{P}_{\mathrm{FO}}= & \text { probability that component is restored following failure } \\
= & 1
\end{aligned}
$$

Our interest lies in obtaining $P_{M D}$ and $P_{D F} \cdot P_{D F}$ describes the effectiveness of maintenance and the probability of transferring to a failed state once a degraded state is reached. $\mathbf{P}_{\mathrm{MD}}$, similarly, expresses the probability of recurring degradation before failure. 
contradicting assumptions 7 and 8 . The discussion in the report is unclear about whether or not assumptions 7 and 8 imply that a degraded state is always detected.

However, a value of $P_{M D}=1$, or equivalently $P_{M F}=0$, would be reasonable. It corresponds to the fact that the repair rate is much greater than the failure rate, and is consistent with the diagram shown in Fig. 2.2.

The starting point in the derivation of quantities of interest is the stationary, steady-state equations relating the failure and degradation frequencies and transition probabilities. By "stationary," it is meant that the probability of a transition from state $i$ to state $j$ during the time interval $(T, T+t)$ does not depend on $T$ but depends only on $t$ and states $i$ and $j$. By "steady state" it is meant that the process has been going on for a "long time" and the asymptotic solution for the process is sought. These equations are derived in the Appendix of the report and are given by

$$
\begin{aligned}
& W_{D}=W_{F}+W_{D} P_{M D} \\
& W_{F}=W_{D} P_{D F},
\end{aligned}
$$

where

$$
\begin{aligned}
& W_{D}=\text { the degradation frequency and } \\
& W_{F}=\text { the failure frequency. }
\end{aligned}
$$

Equations (2.6) and (2.7) yield the following relationships:

$$
\begin{aligned}
& 1=P_{M D}+P_{D F} \\
& P_{D F}=W_{F} / W_{D},
\end{aligned}
$$

Subscript use in the Appendix to describe state transitions appears a bit confusing. It seems that if the authors switched the MD subscript to a DM subscript in equations throughout the Appendix, the equations would be consistent with their diagram in Fig. 2.2

Making the MD to DM subscript change, ${ }^{+}$it appears as through Eqs. (2.6) and (2.7) should read

$$
W_{D}=W_{F}+W_{D} P_{D M}{ }^{*}
$$

and Eq. (2.8) should read

$$
1=\mathrm{PDM}^{*}+\mathrm{PDF}
$$

Although not stated in the derivation in the Appendix, Eqs. (2.6) through (2.11) appear to have the following implicit assumptions embedded in them.

(1) Transition from the $f$ state to the o state is essentially instantaneous, i.e., the transition probability density function is a delta function.

\footnotetext{
${ }^{+}$Subsequent subscript changes from the report are noted by $a *$.
} 
(2) Transition from the $m$ state to the $d$ state is instantaneous, i.e., the transition probability density function is a delta function.

(3) There are no transitions from the $m$ state to the $f$ state.

(4) Transitions from the o state to the $d$ state, the $d$ state to the $m$ state, and the $d$ state to the $f$ state are not instantaneous, and have transition probability density functions that reflect this assumption

(5) $\mathrm{P}_{\mathrm{FO}}=1, \mathrm{P}_{\mathrm{MD}}=1, \mathrm{P}_{\mathrm{MF}}=0, \mathrm{POD}=1$, and $\mathrm{P}_{\mathrm{DM}}{ }^{*}+\mathrm{P}_{\mathrm{DF}}=1$.

Note that in the steady-state equations, $W_{D}$ and $W_{F}$ are constant, as are PDF and PDM*. If data on $W_{D}$ and $W_{F}$ are available, then PDF can be estimated.

A quantity of interest is a measure of maintenance effectiveness. The model suggests that $1-P_{D F}$ is a reasonable measure of the maintenance effectiveness. (PDF is a measure of maintenance ineffectiveness.) The maintenance ineffectiveness for the steady-state case is constant. Basically, the smaller the value of $\mathrm{P}_{\mathrm{DF}}$, the more effective maintenance is.

The authors assume that in the case of time varying $W_{F}(t)$ and $W_{D}(t)$, which they use with actual data, the measure of maintenance ineffectiveness is given approximately by

$$
P_{D F}=\left\langle W_{F}(t)\right\rangle /\left\langle W_{D}(t)\right\rangle,
$$

where $\left\langle W_{F}(t)>\right.$ and $\left\langle W_{D}(t)>\right.$ denote average values over time and are given in the report by

$$
\begin{aligned}
& <W_{F}(t)>=\frac{1}{t_{b}-t_{a}} \int_{t_{a}}^{t_{b}} \lambda_{F}(t) d t \\
& <W_{D}(t)>=\frac{1}{t_{b}-t_{a}} \int_{t_{a}}^{t_{b}} \lambda_{D}(t) d t,
\end{aligned}
$$

where $\lambda_{D}(t)$ and $\lambda_{F}(t)$ are the time-dependent degradation and failure rate, respectively. Equation (2.13) appears incorrect, and should have $W_{F}(t)$ and $W_{D}(t)$ as integrands, respectively. Remaining discussion assumes that the integrands are $W_{F}(t)$ and $W_{D}(t)$.

The report goes on to discuss the time dependent case, and in particular the case where the transition probability density functions are exponential with parameters characterizing the transition rates from $d$ to $f$ (failure rate), $\lambda_{D F}$, from o to $d$ (operational degradation rate), $\lambda_{O D}$, and $d$ to $\mathrm{m}$ (degradation rate) $\lambda \mathrm{DM}^{*}$. The functional form of these transition probability density functions is given by

$$
\begin{aligned}
& f_{D F}(x)=\lambda D F \exp \left(-\lambda_{M}\right) \\
& f_{D M}(x)=\lambda_{D M^{*}} \exp \left(-\lambda_{M}\right) \\
& f_{O D}(x)=\lambda O F \exp \left(-\lambda_{O D}\right)
\end{aligned}
$$

where

$$
\lambda_{\mathrm{D}^{*}}=\lambda_{\mathrm{DM}^{*}}+\lambda_{\mathrm{DF}}
$$


The authors calculate the following steady-state maintenance ineffectiveness,

$$
\mathrm{PDF}_{\mathrm{DF}}=\lambda_{\mathrm{DF}} /\left(\lambda_{\mathrm{DF}}+\lambda_{\mathrm{DM}}\right)^{*}=\mathrm{WF}_{\mathrm{F}} / \mathrm{W}_{\mathrm{D}}
$$

based on the steady-state value of PDF given by

$$
P_{D F}=\int_{0}^{\infty} f_{D F}(t-x) d x
$$

Although the authors do not actually solve the time dependent equations in the Appendix for the assumed exponential distributions, a solution is possible and is given by

$$
\begin{aligned}
& \mathrm{W}_{\mathrm{F}}(\mathrm{t})=\frac{\lambda_{\mathrm{DF}} \lambda_{\mathrm{OD}}}{\lambda_{\mathrm{OD}}+\lambda_{\mathrm{DF}}}\left\{1-\exp \left[-\left(\lambda_{\mathrm{OD}}+\lambda_{\mathrm{DF}}\right) \mathrm{t}\right]\right\} \text { and } \\
& \mathrm{W}_{\mathrm{D}}(\mathrm{t})=\frac{\lambda_{\mathrm{D}} \lambda_{\mathrm{OD}}}{\lambda_{\mathrm{OD}}+\lambda_{\mathrm{DF}}}+\left(\lambda_{\mathrm{OD}}-\frac{\lambda_{\mathrm{OD}} \lambda_{\mathrm{D}}}{\lambda_{\mathrm{OD}}+\lambda_{\mathrm{DF}}}\right) \exp \left[-\left(\lambda_{\mathrm{OD}}+\lambda_{\mathrm{DF}}\right) \mathrm{t}\right],
\end{aligned}
$$

where it has been assumed that the threshold time to $=0$.

Equations (2.17) and (2.18) lead to the following steady-state solutions for $W_{F}$ and $W_{D}$ :

$$
\begin{aligned}
& \mathrm{W}_{\mathrm{F}}=\frac{\lambda_{\mathrm{DF}} \lambda_{\mathrm{OD}}}{\lambda_{\mathrm{OD}}+\lambda_{\mathrm{DF}}} \text { and } \\
& \mathrm{W}_{\mathrm{D}}=\frac{\lambda_{\mathrm{D}} \lambda_{\mathrm{OD}}}{\lambda_{\mathrm{OD}}+\lambda_{\mathrm{DF}}} .
\end{aligned}
$$

Therefore, Eq. (2.15) is obtained as a steady-state condition. However, note that if Eq. (2.12) is to be used to estimate the time-dependent maintenance ineffectiveness by allowing for time dependent $\lambda$ 's, it appears as if several time-dependent terms should be included in the integrand of Eq. (2.13) for completeness. This is because the authors are using a ratio of the averages, each piece of which contains terms that are divided out when the ratio is calculated directly without averaging. Thus, one is led to question their calculated results for maintenance ineffectiveness with time varying $\lambda$ 's using their definition of maintenance ineffectiveness. It is tempting to redefine the maintenance ineffectiveness as an average of the ratio, i.e.,

$$
\text { Maintenance Ineffectiveness }=\left\langle W_{F} / W_{D}\right\rangle \text {, }
$$

rather than as the ratio of the averages as in Eq. (2.12).

Another point concerns how good a measure of maintenance effectiveness is $1-W_{F} / W_{D}=$ $1-\mathrm{PDF}$. PDF can be small because $\lambda_{\mathrm{DF}}$ is small, but it also can be small because $\lambda_{\mathrm{DM}}{ }^{*}$ is large. In the latter case, the system may be spending an inordinate time in maintenance, and gains made by reducing the probability the system is in a filed state at the time of a demand, is lost because of the increased maintenance unavailability of the system.

The discussion will now turn from maintenance effectiveness to that of incorporating aging effects into the degradation modeling. The authors basically describe aging as allowing the various transition frequencies, the $\lambda$ 's, to be functions of time. 
Analysis of this case is not developed in detail in the model other than to acknowledge its existence and suggest an approach to estimating the failure rate in the absence of failure data.

What the authors essentially have done, in an attempt to incorporate aging effects, is to assume that Eqs. (2.12) and (2.15) are valid even if the various rates are similar functions of time, namely,

$$
\begin{aligned}
& \lambda_{D F}(t)=\operatorname{kDFg}(t) \text { and } \\
& \lambda_{D M^{*}}(t)=k_{D M^{*}} g(t),
\end{aligned}
$$

where $g(t)$ is a general time-dependent function and $\mathrm{kDF}$ and $\mathrm{kDM}^{*}$ are constants.

This is an attempt to use the steady-state value of the ratio in Eq. (2.15), which was derived under the assumption of exponential transition probability density functions, as the basis for estimating the aging failure rate from the aging degradation rate. It has been assumed that Eq. (2.15) is a reasonable approximation for all time even if time-dependent $\lambda$ 's are used. Thus, PDF will remain a constant $=k_{D F} /\left(k_{D F}+k_{D M}\right)$ if the functional forms of Eq. (2.15) are used, and an estimate can be made of the effect of aging on the failure rate through knowledge of the steady-state ratio, PDF, calculated from steady-state $W_{F}$ and $W_{D}$ values.

It is true that the limiting state probabilities for systems with time-dependent transition rates, the $\lambda$ 's, will depend only on the mean values of the probability distributions describing these transition rates (Billinton and Allan, 1983). However, this information cannot be used to determine the functional form of the underlying time dependent transition rate. Whether or not data are available, some insight into the physical mechanisms governing a component's life, must be brought into the analysis.

This model is theoretical and has not been tested as a predictor. The data analyzed in the report show that the degradation and failure rates do not move in tandem over time, i.e., Eq. (2.22) is not true in general. This leads to questions about the accuracy of using the degradation rate to predict the failure rate as the component ages and the rates vary with time. As mentioned above, an effort to incorporate physical phenomena causing degradation and failure in the component into an aging model is required if the model is to be accurate.

Another potential problem is in the modeling of small degradation and failure rates. Using steady-state values in the model may not be accurate because enough time may not have passed for the steady-state approximation to be valid.

As a final comment on the model, the lack of a catastrophic failure mode prevents certain shock phenomena from being modeled.

\subsubsection{Comments on Degradation Modeling}

Degradation modeling applies methods of statistical inference to predict and describe different modes between failure and perfect operation of test items that commonly display signs of wear or aging that are precursors to failure. This type of modeling reaches beyond the scope of basic reliability and failure rate analysis and is not amply described or investigated in the literature. Even though the reliability degradation model discussed in NUREG/CR-5612 is essentially as simple as possible (with only one level of degradation used), the study of the stochastic modeling of an item's lifetime degradation can prove to be very complicated. In general, such modeling increases the range of output from binary (success and failure conditions only) to ternary (with an added condition of degradation) or possibly an infinite number of levels of degradation for the case in which a continuous scale of degradation is used for a more realistic model. As an example, certain electronic components exhibit 
measurable levels of degradation before failure, so multistep failure modes can be used to model the component lifetime [LuValle (1992)]. Other examples can be found in Bogdanoff and Kozin (1985) and Klinger (1992).

To use degradation modeling, the methods and quality of data collection must be extremely thorough. Failure descriptions and maintenance procedures for components require great detail, more than the typical descriptions of failure time and failure mode. If component degradation is identified as a state between failure and non failure, some component state or range of states must be designated for the defined degraded condition(s). Otherwise, interpretation of reliability maintenance records may lead to ambiguous results. For example, a component undergoing a routine maintenance procedure and another component experiencing incipient failure may both share a degraded reliability classification, even though there are great differences in the degree of unreliability in such components. Any set of objective rules for degradation reliability classification would likely change for different component types and their various uses and environments. As a consequence, the collection of reliability data for component groups could be much more complicated and time-consuming than current procedures.

The specific application of reliability degradation modeling in NUREG/CR-5612 is most likely not the best source for use in future reliability studies. The mathematical and statistical formulations (especially in Sec. 4) are difficult to verify, and the motivation for the chosen methods is not always clear. In particular, the application of the Cox proportional hazards model is questionable for the posed problem. The Cox model is a nonparametric generalization of the life-table and KaplanMeier estimators of the underlying distribution function [see Kaplan and Meier (1958) or Miller (1981) for discussions of these]. It models the unknown failure rate function as a function of time and a set of covariates related to the failure distribution. The Cox model is especially useful in cases of control studies and other biostatistical applications in which covariates help partition treatment groups and population effects. Examples are discussed and referenced in Prentice and Kalbfleisch (1979), Efron (1977), and Miller (1981). The Cox model is not described in any detail here, but one attractive feature of the proportional hazards modeling worth mentioning is that linear regression sometimes can be used in parameter estimation, including this particular application in NUREG/CR-5612. However, the problem presented in that report is already fully parameterized, and applying the Cox model only exploits the apparent simplicity of the regression technique used to estimate the log failure rate of the component lifetimes. The end product is an easily applied regression procedure that can be deployed with various statistical software packages, but it is not clear that the Cox model is well suited for this problem. Parametric models appear to be best analyzed using parametric procedures, as in NUREG/CR-5378. Further review of the application of the Cox model is difficult because the description of the data is abbreviated.

Pooling is desirable in many problems when there are small groups of data for similar components. Possible pooling of data according to similarity of component groups is determined in NUREG/CR-5612 using nonparametric tests (Kruskal-Wallis, Mann-Whitney). Unlike the parametric tests and procedures used in a similar way in NUREG/CR-5378, these tests are less likely to misinform the user in the case the strict assumptions of the statistical model are not perfectly met.

Isotonic regression is applied to the analysis along with the nonparametric tests. Isotonic regression is used for constrained estimation problems (e.g., estimating a failure rate that is known to be increasing in time), but it is unclear how the regression is applied to the data or even what constraint is used in the model. Therefore, this use of isotonic regression for RHR pump data (see p. D-1 of NUREG/CR-5612) is questionable. 


\subsection{Component Failure Rate Models and Reliability Distributions}

\subsubsection{Introduction}

The reliability of a system or component is defined as the probability that the system or component will perform its intended function adequately under stated environmental conditions for a specified interval of time. The probability of failure of a system or component as a function of time is defined as

$$
\mathrm{P}(\mathrm{T} \leq \mathrm{t})=\mathrm{F}(\mathrm{t})
$$

where $T$ is a random variable representing the failure time and $F(t)$ is the cumulative distribution function (cdf) of $T$. The reliability of the system is then

$$
R(t)=1-F(t)=P(T>t),
$$

where $R(t)$ is the reliability function. The failure density, $f(t)$, is the probability density function, (pdf), of $F(t)$, i.e.,

$$
f(t)=\frac{d F(t)}{d t}
$$

The failure rate, which also is called the hazard function, is given by

$$
\lambda(t)=\frac{f(t)}{R(t)} .
$$

The failure rate is interpreted as follows. $\lambda(t) \mathrm{dt}$ is the conditional probability of failure in the interval $(t, t+d t)$ given that the system is functioning at $t$. Note the difference from the pdf, which is interpreted as $f(t) d t$ is the unconditional probability of failure in the interval $(t, t+d t)$. Equations (2.24)-(2.26) lead to

$$
R(t)=\exp \left[-\int_{0}^{t} \lambda\left(t^{\prime}\right) d t^{\prime}\right]
$$

In general, the failure rate changes over time as shown in Fig. 2.4, which depicts the so-called "bathtub" curve. The first interval, from time $t_{0}$ to time $t_{1}$, represents early failures because of material or manufacturing defects and usually is referred to as the "burn-in" period or the "infant mortality" period. The second period, from time $t_{1}$ to time $t_{2}$, is the period of random failures and is usually approximated adequately by a constant failure rate. It often is referred to as the "useful life" of the component or system. The portion of the curve after $t_{2}$ represents so-called "wear-out" failures, where the failure rate increases as the component deteriorates. With respect to aging, this is the period of the component's life that is of concern.

One way to formulate a reliability model is to describe the physical phenomena leading to failure and then use these phenomena as a logical basis for derivation of the reliability distribution. Unfortunately, this can be a difficult task. In later sections of this report, such physically based approaches will be described and discussed for selected degradation mechanisms. Models of this type are known as "reliability physics" models or "stress-strength interference" models. 


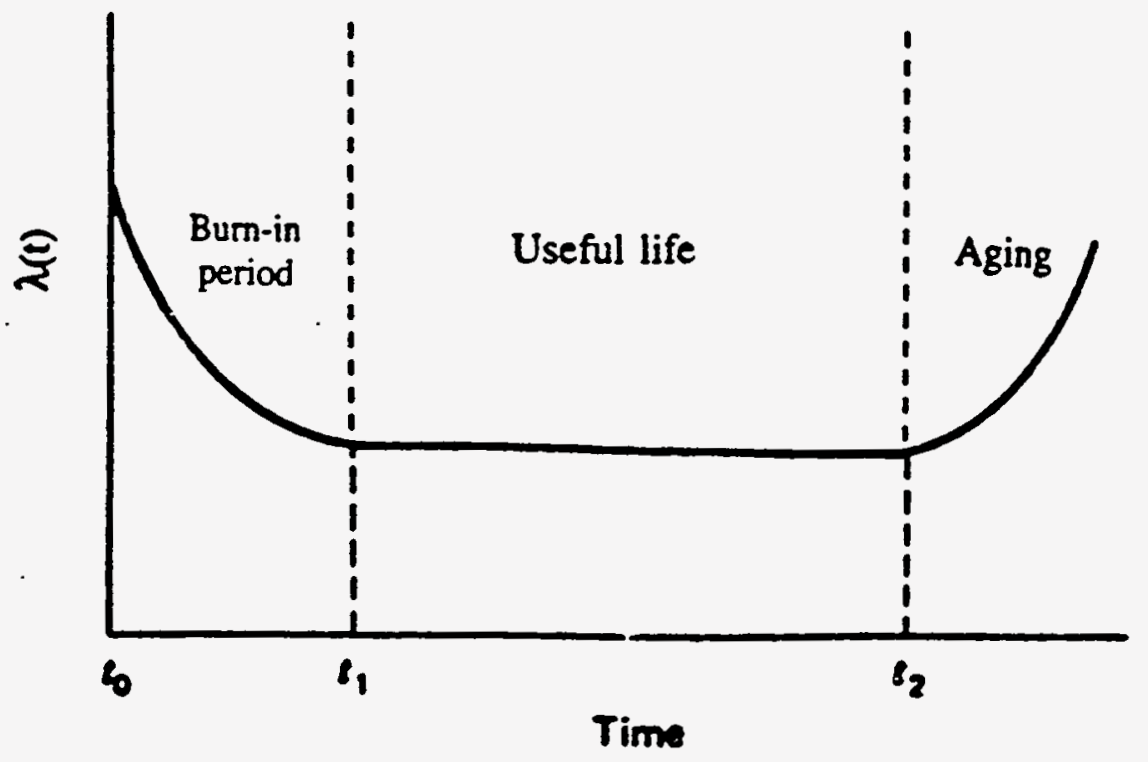

Figure 2.4 Failure rate as a function of time 
An alternate approach is to assume a distribution for the reliability of the system and to estimate the parameters of the distribution from lifetime data using appropriate statistical techniques. Data generally come from a group of similar components, and it is assumed that all components have the same failure rate and that failure of any component is independent of failures of the other components. Thus, in these so-called "parametric models," the physical mechanisms leading to failure are not considered explicitly. Rather, a specific functional form of the failure rate [Eq. (2.26)] is assumed, and the reliability is calculated from Eq. (2.27). The principal model proposed in the NPAR program, that of a failure rate increasing linearly in time, falls into this class of models.

In this chapter, some of the more common reliability distribution models will be discussed, including the linearly increasing failure rate model. For each model, we will state its objectives, present its formulation, and describe its basic assumptions.

\subsubsection{Common Reliability Distributions}

\subsubsection{Exponential Distribution}

\section{Model Formulation}

The exponential distribution is widely used in reliability and PRA and is given by

$$
f(t)=\lambda e^{-\lambda t}
$$

the reliability function is given by

$$
R(t)=e^{-\lambda t}
$$

The form of the exponential density function is shown in Fig. 2.5. The failure rate of the exponential distribution is constant and is given by

$$
\lambda(t)=\frac{f(t)}{R(t)}=\lambda .
$$

\section{Model Assumptions}

The basic assumption is that the failure rate is constant; i.e., there is no deterioration or improvement of the item over time.

\section{Discussion}

The exponential reliability distribution is the most commonly used distribution in life testing applications and generally is used in PRAs to model basic component reliability to estimate system unavailabilities. In many cases, it is used because it is easy to apply, rather than being based on a thorough understanding of physical fundamentals.

\subsubsection{Normal Distribution}

\section{Model Formulation}

The normal distribution is the familiar bell-shaped curve. The normal density function is given 


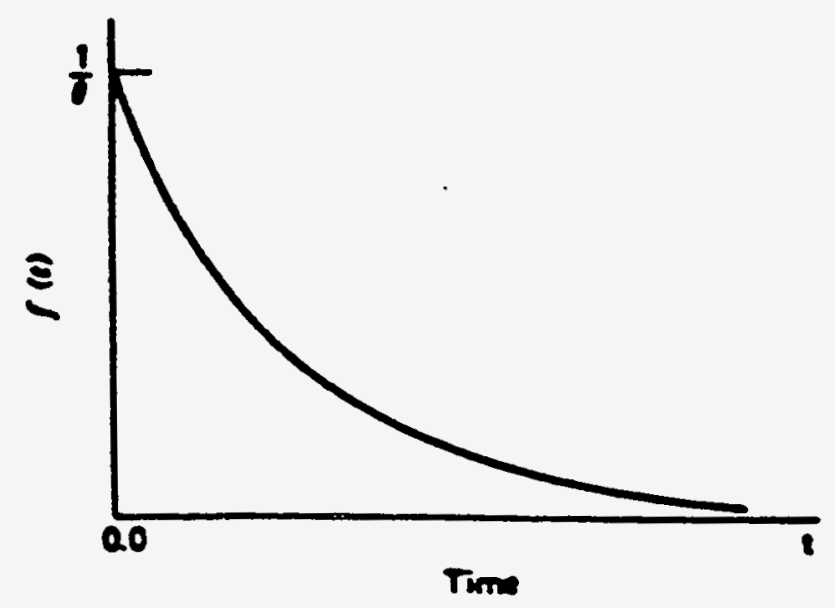

Figure 2.5 The exponential failure density function 


$$
f(t)=\frac{1}{\sqrt{2 \pi}} \exp \left[-\frac{1}{2}\left(\frac{t-\mu}{\sigma}\right)^{2}\right]
$$

where $\mu$ is the mean and $\sigma$ is the standard deviation. The reliability distribution is then

$$
R(t)=\int_{t}^{\infty} f\left(t^{\prime}\right) d t^{\prime}=1-\Phi\left(\frac{t-\mu}{\sigma}\right),
$$

where $\Phi(z)$ is the standard normal cumulative distribution function. The normal reliability function is shown in Fig. 2.6. The failure rate then is given by

$$
\lambda(\mathrm{t})=\frac{\exp \left[-\frac{1}{2}\left(\frac{\mathrm{t}-\mu}{\sigma}\right)^{2}\right]}{\int_{\mathrm{t}}^{\infty} \exp \left[-\frac{1}{2}\left(\frac{\mathrm{t}^{\prime}-\mu}{\sigma}\right)^{2}\right] d \mathrm{t}^{\prime}} .
$$

and is shown in Fig. 2.7. The failure rate is a monotonically increasing function of time. Note that as $t$ becomes very large, the limit of $\lambda(t)$ can be calculated by invoking L'Hospital's rule to get

$$
\lim _{t \rightarrow \infty} \lambda(t)=\frac{t-\tau}{\sigma}
$$

Thus, for large $t$, the failure rate increases linearly with time.

\section{Model Assumptions and Discussion}

The distinctive characteristic of this model is the symmetry of its pdf. The failure rate is an increasing function of time, thus, it may be used as an aging model. In fact, as shown above [Eq. (2.34)], the failure rate tends to increase linearly with time for large $t$.

\subsubsection{Lognormal Distribution}

\section{Model Formulation}

The lognormal distribution arises when the natural logarithm of the failure time is normally distributed. The lognormal probability density function is given by

$$
f(t)=\frac{1}{\sigma t \sqrt{2 \pi}} \exp \left[-\frac{1}{2}\left(\frac{\ln t-\mu}{\sigma}\right)^{2}\right]
$$

and the failure rate is given by 


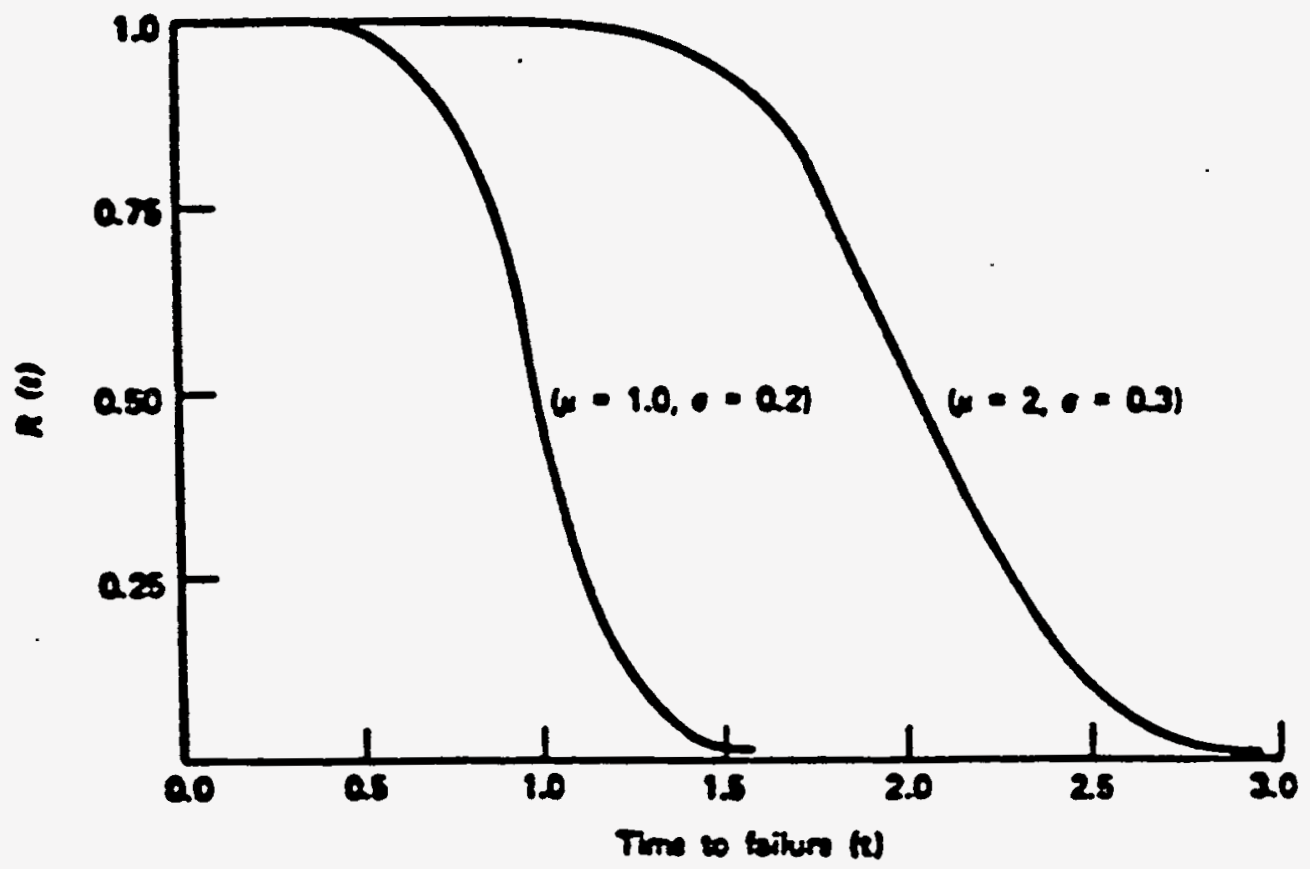

Figure 2.6 The normal reliability distribution 


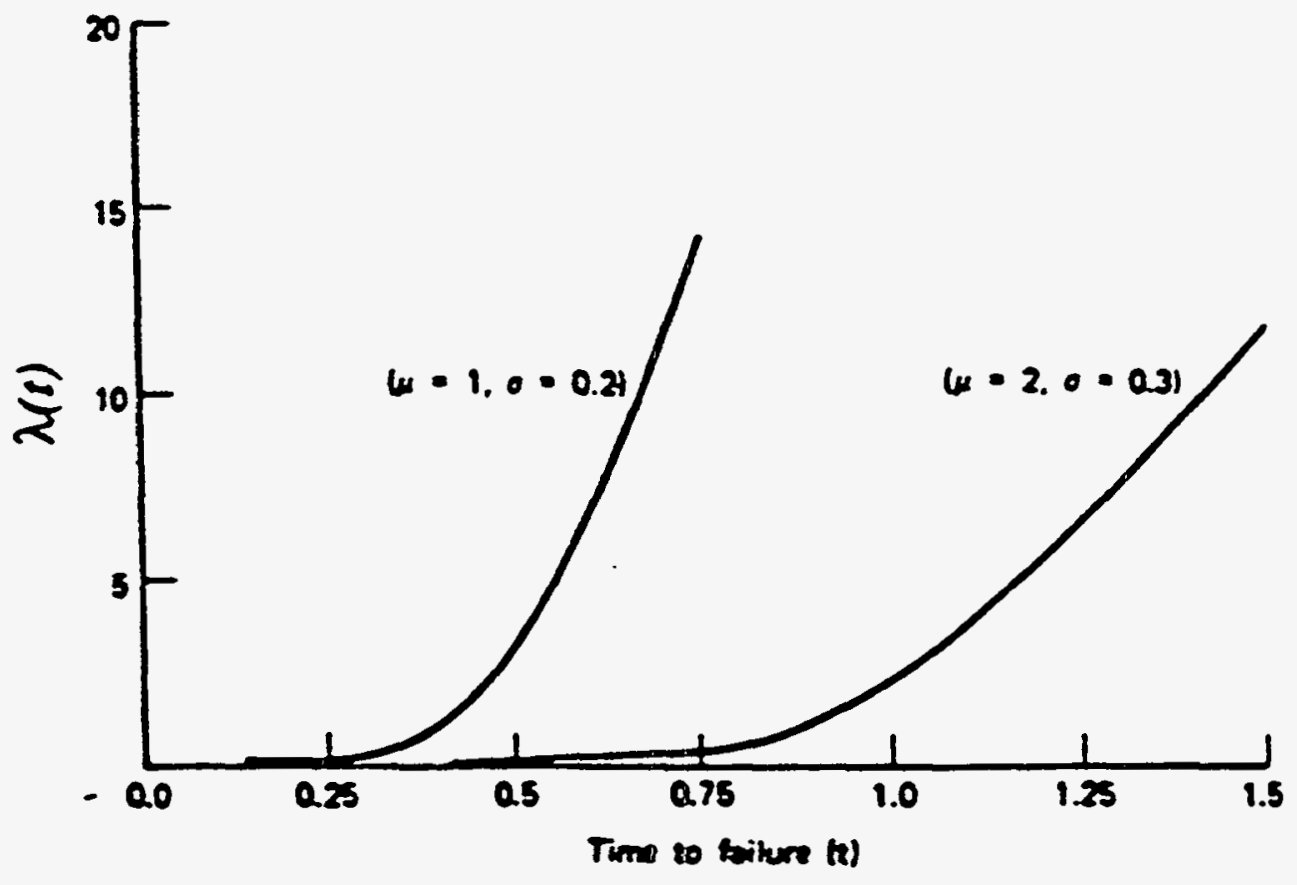

Figure 2.7 The normal failure rate 


$$
\lambda(t)=\frac{\frac{1}{t} \exp \left[-\frac{1}{2}\left(\frac{\ln t-\mu}{\sigma}\right)^{2}\right]}{\int_{t}^{\infty} \frac{1}{t^{\prime}} \exp \left[-\frac{1}{2}\left(\frac{\ln t^{\prime}-\mu}{\sigma}\right)^{2}\right] d t}
$$

The failure density, reliability distribution, and failure rate are shown in Figs. 2.8 through 2.10. Again, by application of L'Hospital's theorem, the behavior of the lognormal distribution failure rate can be shown to follow the functional form

$$
\lim _{t \rightarrow \infty} \lambda(t)=\frac{1}{t}\left[1+\frac{\ln (t / \tau)}{\sigma^{2}}\right]
$$

for large $t$ values.

Thus, for large $t$, the failure rate decreases to zero after reaching a maximum value earlier in the component life.

\section{Model Assumptions and Discussion}

The failure rate, $\lambda(t)$, initially increases with time, reaches a maximum, and then goes to zero. Thus, the lognormal distribution can, in principle, be used to model aging over the range where the failure rate is increasing with time.

\subsubsection{Weibull Distribution}

\section{Model Formulation}

The distribution of the time to failure can be written as

$$
F(t)=1-\exp \left[-\left(\frac{t-\gamma}{\eta}\right)^{\beta}\right]
$$

and the failure rate is given by

$$
\lambda(t)=\beta \frac{(t-\gamma)^{\beta-1}}{\eta^{\beta}},
$$

where $t$ is the time to failure, $\gamma$ is the time at which $F(t)=0$ (a location parameter), $\eta$ is the characteristic life (the scale parameter), and $\beta$ is the shape parameter. If $\beta<1, \lambda(t)$ is a decreasing function of time; if $\beta=1, \lambda$ is constant in time and if $\beta>1, \lambda(t)$ is an increasing function of time. In particular, for $\beta=2$, the failure rate is increasing linearly with time, so that the Weibull distribution closely resembles the linear aging model for this particular value of $\beta$. The forms of the failure density, reliability distribution, and failure rate are illustrated in Figs. 2.11-2.13.

\section{Model Assumptions}

This model assumes that the failure rate changes as some power $(\beta-1)$ of time. 


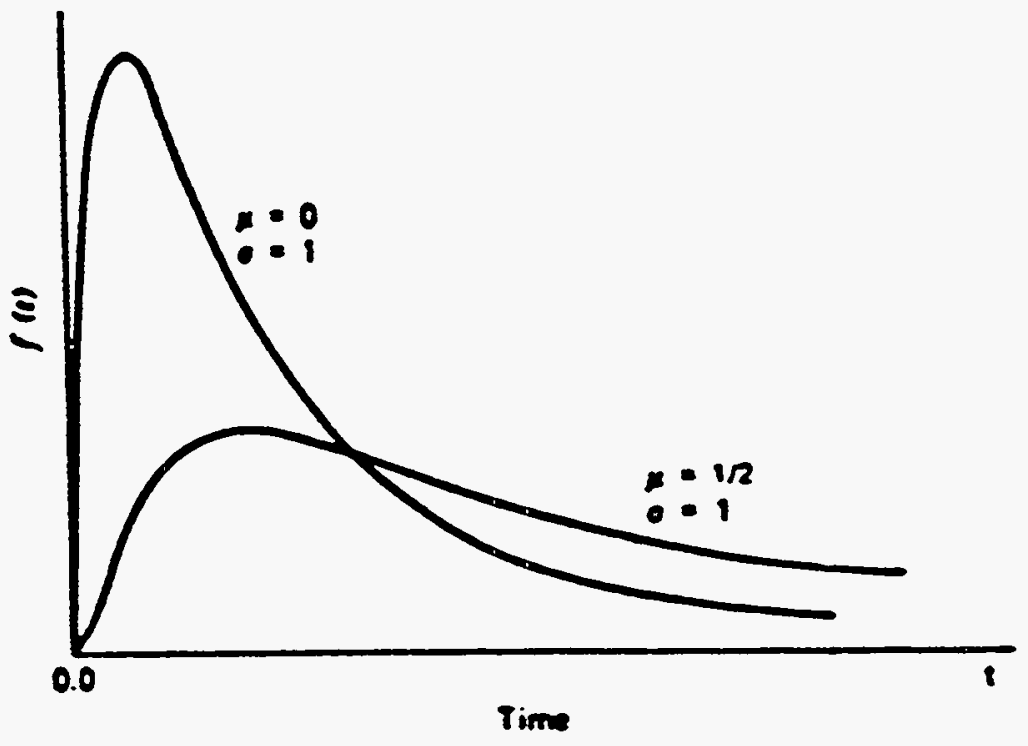

Figure 2.8 The lognormal failure density function 


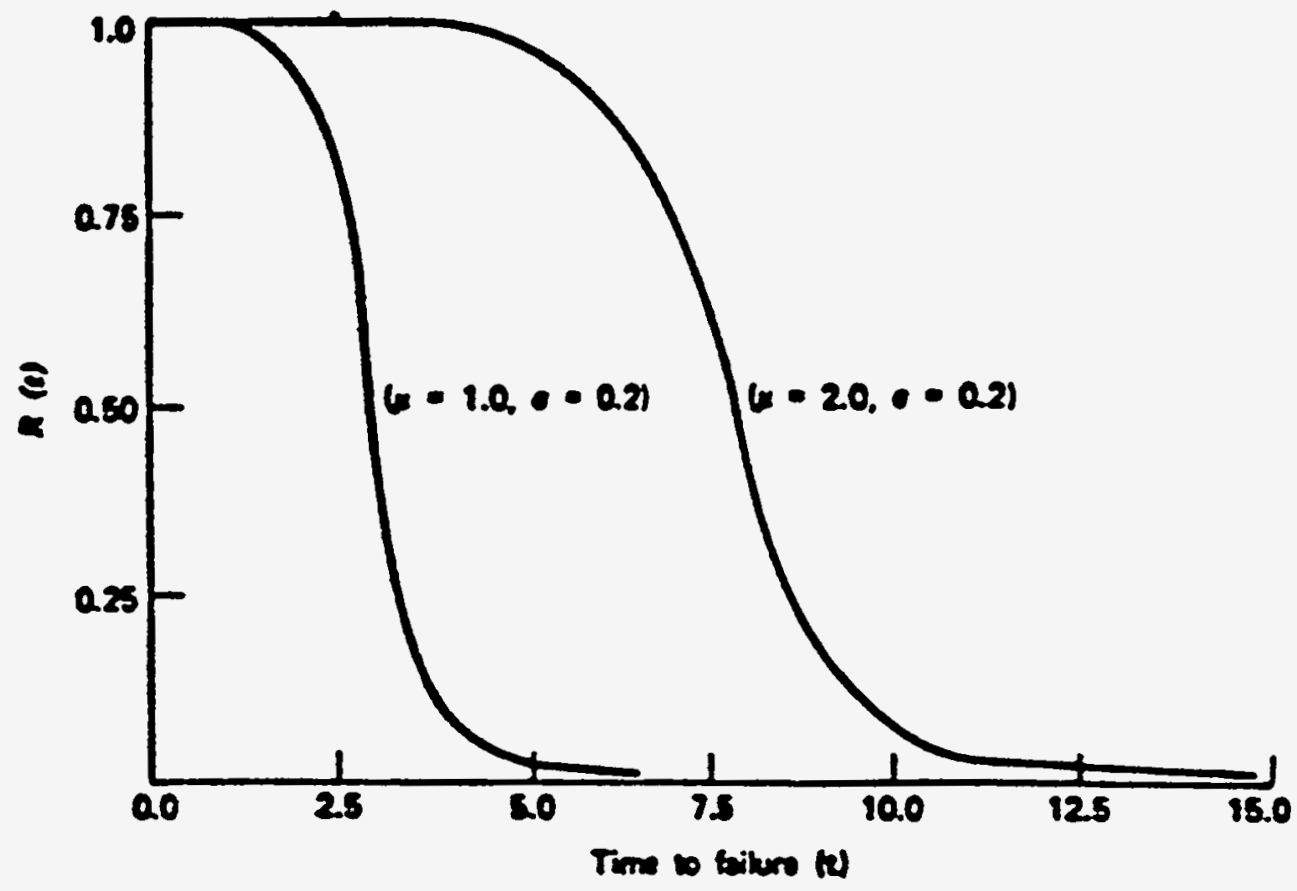

Figure 2.9 The lognormal reliability distribution 


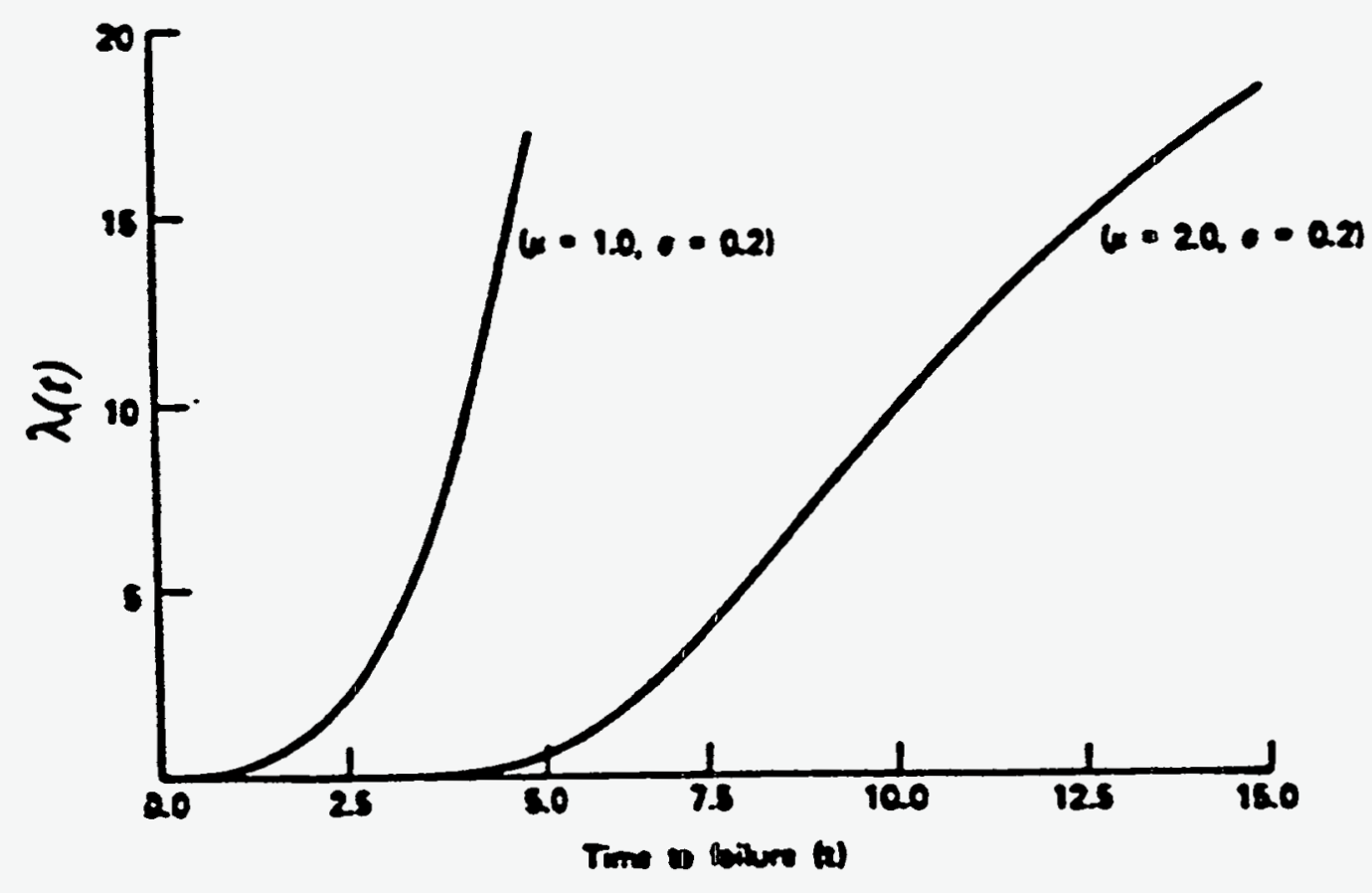

Figure 2.10 The lognormal failure rate 


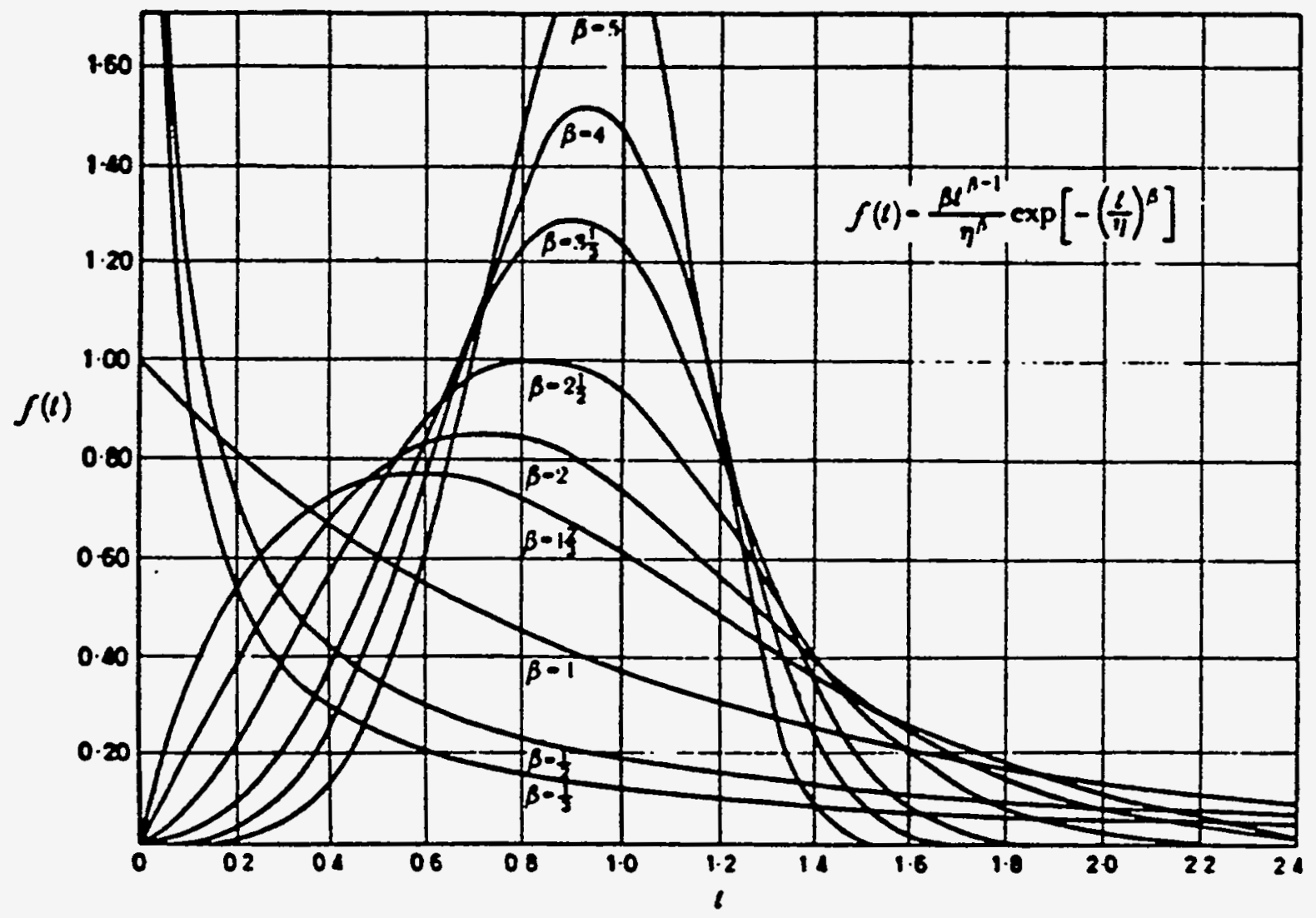

Figure 2.11 The Weibull probability density function for various values of $\beta$ when $\eta=1, \gamma=0$ 


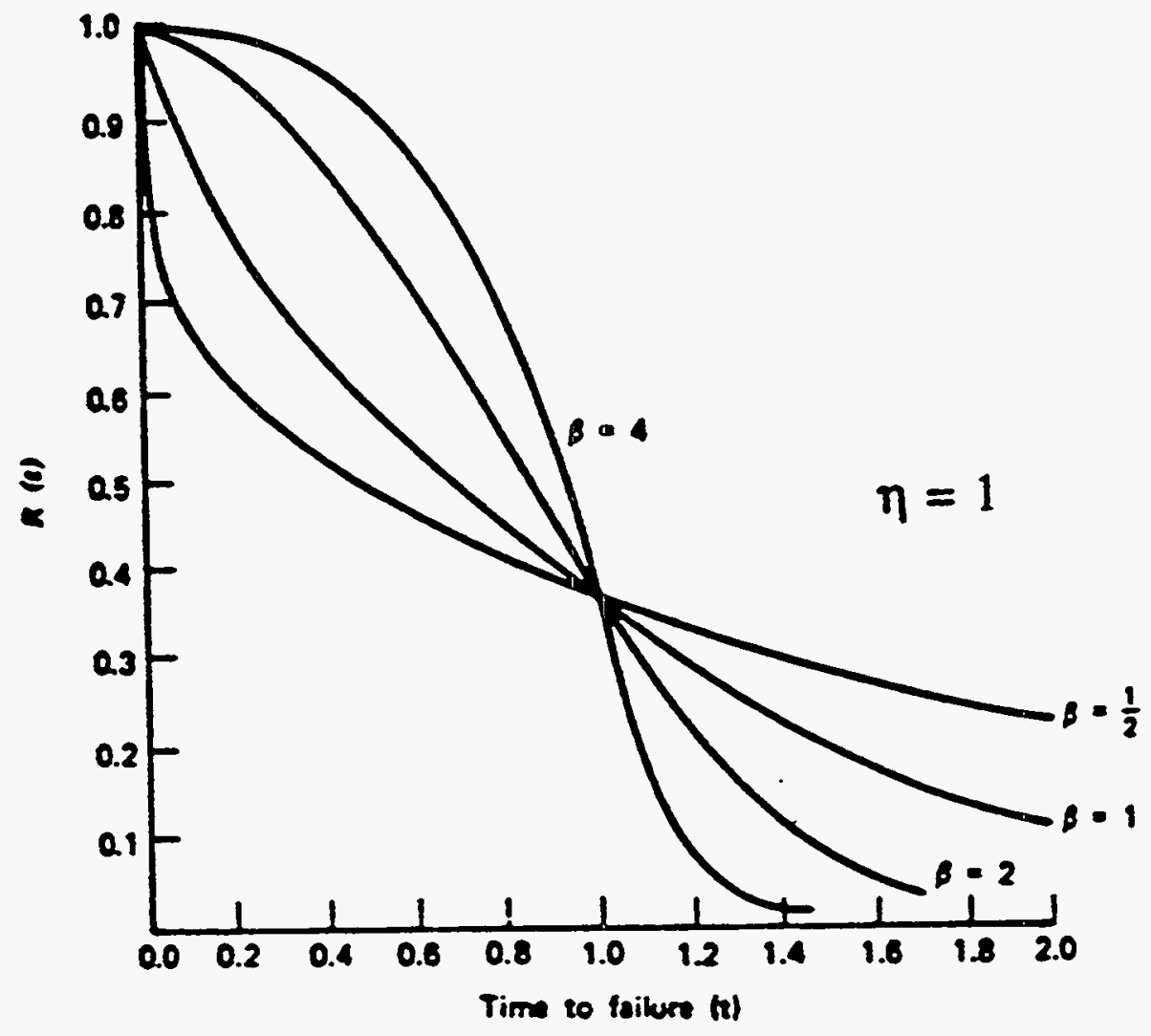

Figure 2.12 The Weibull reliability distribution 


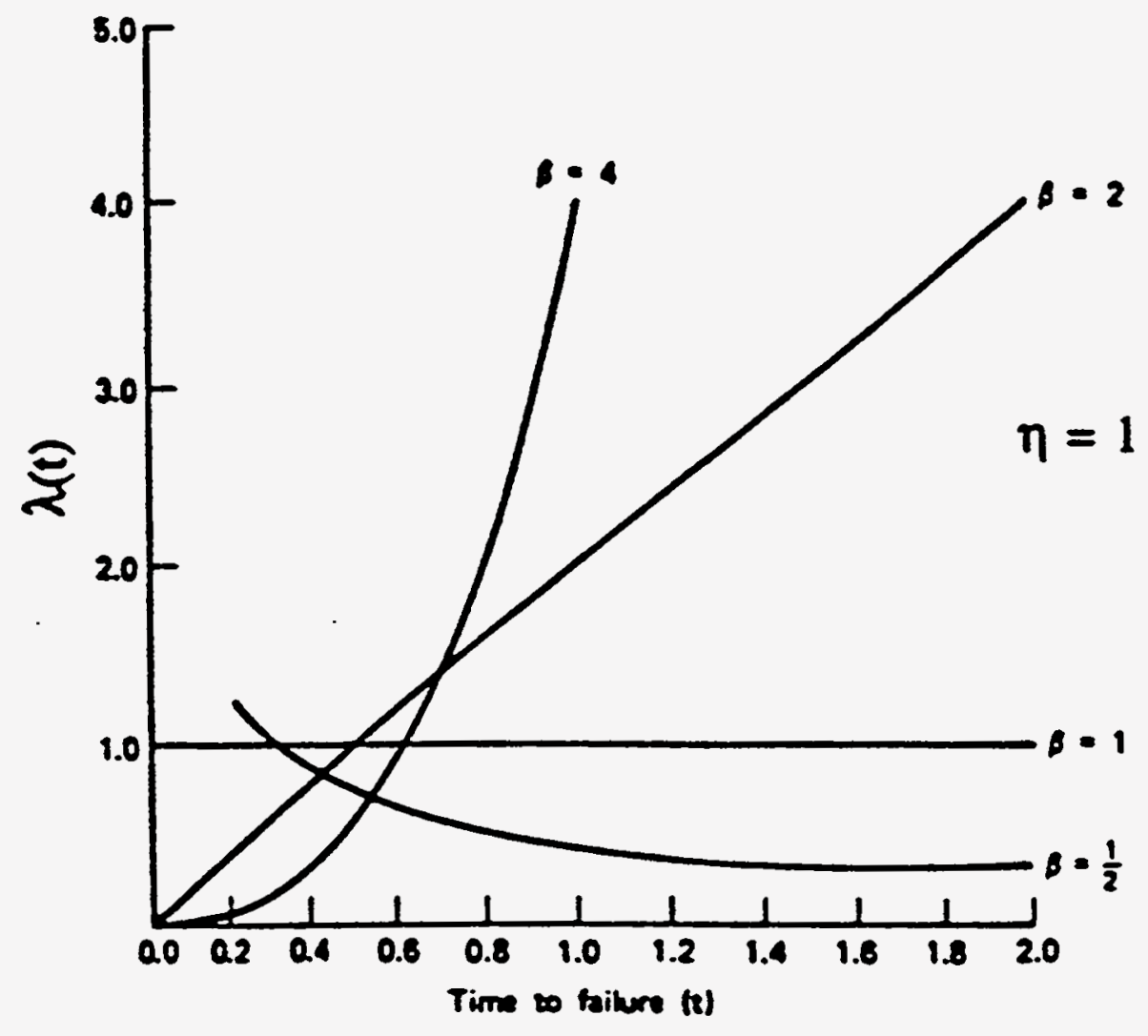

Figure 2.13 The Weibull failure rate 


\section{Discussion}

The Weibull distribution is used commonly to model fatigue lifetime and was devised to describe the results of fatigue tests. It embraces a great variety of forms, including the exponential distribution and one that closely approximates the normal distribution. This model often is used to model the reliability of electrical components subject to wear-out and is reviewed in Engelhardt (1988). It can be used to describe both the burn-in phase and the wear-out phase of the bathtub curve and the useful life because the exponential distribution is a special case of the Weibull distribution.

\subsubsection{Extreme Value Distribution}

\section{Model Formulation}

Because material or equipment failure often is related to the weakest point or the weakest component, the extreme value distribution is sometimes used as a reliability distribution. The density function of the extreme value distribution is given by

$$
f(t)=\frac{1}{\beta} \exp \left(\frac{t-\alpha}{\beta}\right) \exp \left[-\exp \left(\frac{t-\alpha}{\beta}\right)\right],
$$

and the reliability distribution is

$$
R(t)=\exp \left[-\exp \left(\frac{t-\alpha}{\beta}\right)\right]
$$

The failure rate is then an exponential function of time, i.e.,

$$
\lambda(t)=\frac{1}{\beta} \exp \left(\frac{t-\alpha}{\beta}\right) .
$$

\section{Model Assumptions}

This model assumes that the failure rate is an exponential function of time.

\section{Discussion}

The extreme value distribution arises when a component is composed of many similar elements, and if one of the elements fails, then the component fails. If the probability density function for the strength of the elements is approximated by a density function that has a left-handed tail of the exponential type, then the distribution of the weakest element approaches the extreme value distribution as the number of elements increases. A distribution of the exponential type on the left means, roughly speaking, that the variate is unbounded on the left and that it converges to zero on the left at least as fast as the exponential function. For a precise definition of the term "exponential type," see Gumbel (1958). If the damage to each element accumulates linearly in time and at the same rate for each element, it can be shown that the failure time has the extreme value distribution. This model has been considered in detail by Cox and Lewis (1966) and Lawless (1982).

\section{Multimodal Distributions}

Multimodal, or mixed, failure distributions arise when there is a multimodal strength or stress distribution, or when there are different modes of failure of the component. The bathtub curve often is modeled as a mixture of three Weibull distributions-one with a decreasing failure rate, one with an 
increasing failure rate, and one with a constant failure rate. These represent the three modes of failure: early failures because of defects in materials or manufacturing, random failures, and failures resulting from wear-out or aging. Bompas-Smith (1973) contains a good discussion of multimodal reliability distributions.

\subsubsection{The Linear Aging Reliability Model (Component Aging Reliability Model)}

\subsubsection{The Model's Objective}

The objective of the linear aging reliability model is to include the effects of aging in the reliability assessment of individual components. The revised component failure rates then can be used later in PRA models to determine how system unavailabilities, core melt frequency, and public risks change with plant age.

With the scarcity of existing failure data that exhibit aging behavior characteristics, another objective of the linear aging reliability model is to provide a simple framework that is compatible with sparse data but still can provide meaningful engineering insights.

\subsubsection{The Model's Formulation}

In the linear aging reliability model, the effects of the aging process on the component failure probability are expressed as a simple linear equation that relates the component failure rate to the exposure time of the component to the aging mechanism (component age). The failure rate is written as

$$
\lambda(t)=\lambda_{0}+\alpha t
$$

and the component reliability function is given by

$$
R(t)=e^{-\lambda_{0} t} e^{-\alpha \frac{t^{2}}{2}}
$$

The linear failure rate model described above assumes that the total failure rate, $\lambda(t)$, is the sum of two independent failure rates, one associated with random failures, $\lambda_{0}$, and associated with failures caused by aging, $\alpha$ t. In this sense, the linear failure rate model can be considered to be a mixture of two Weibull distributions, one with $\beta \approx 1$ and one with $\beta \approx 2$. Thus, the distribution of time to failure could be written as

$$
F(t)=1-\exp \left[-\left(\frac{t}{\eta_{1}}\right)^{\beta_{1}}\right] \exp \left[-\left(\frac{t}{\eta_{2}}\right)^{\beta 2}\right],
$$

where $\beta_{1} \approx 1$ and $\beta_{2} \approx 2 . \eta_{1}$ is approximately the mean time to random failure, and $\left(1 / \eta_{2}\right)^{2}$ is approximately $\alpha / 2$, the constant associated with aging-related failures. If lifetime data for components suspected to be experiencing aging-related degradation are plotted on Weibull paper, and the graph shows two curves that are approximately linear, one at early times with a slope of about 1 (associated with random failures) and another at later times where the aging term dominates, with a slope of about 2 , it can be concluded that the component is exhibiting aging-related degradation and that the linear failure rate model is a good approximation. Recalling the observations made in Sec. 2.4.2.2, it also can be observed that the linearly increasing portion of the rate leads asymptotically to a normal failure distribution. 


\subsubsection{Assumptions}

The major assumption of this model is Eq. (2.43), which defines the dependence of the failure rate on time. An attempt at a physical justification of the linearly increasing part of the failure rate is described in Vesely (1988). In that derivation, it is assumed that the component failure rate is directly proportional to the specific amount of deterioration, $D$, that the component has experienced from a specific aging mechanism up to age t, i.e.,

$$
\lambda(\mathrm{t})=\mathrm{kD} \text {, }
$$

where $k$ is a constant.

The amount of deterioration, $D$, is assumed to be random in both the times at which the deteriorations occur and the severity of damage incurred by the component each time a deterioration occurs. With further assumptions that the occurrence of the deterioration can be described by a Poisson process with a constant occurrence rate, $r$, and the severity of the deterioration can be described by a pdf $f(x)$, the expected value for $\lambda(t)$ can be expressed as

$$
\bar{\lambda}(t)=\sum_{n=0}^{\infty} P_{n}(t) \int_{0}^{\infty} f_{n}(D) k D d D,
$$

where $P_{n}(t)$ is the probability that $n$ deteriorations occur by age $t$ and $f_{n}(D)$ is the probability distribution for the total deterioration, $D$, given that $n$ deteriorations have occurred. It is assumed that the total deterioration, $D$, is the sum of the $n$ independent deteriorations, $x$. Hence, $f_{n}(D)$ is the $n$-fold convolution of the individual distributions, $f(x)$. Thus, $\bar{\lambda}$ becomes

$$
\bar{\lambda}(\mathrm{t})=\mathrm{k} \overline{\mathrm{x}} \mathrm{rt}
$$

where

$$
n \bar{x}=\int_{0}^{\infty} f_{n}(D) D d D
$$

and

$$
\bar{x}=\int_{0}^{\infty} f(D) D d D
$$

If we let $\alpha=k \bar{x} r$, we get

$$
\bar{\lambda}(\mathrm{t})=\alpha \mathrm{t}
$$

which is the basic expression for the component failure rate in the linear aging reliability model.

In an extended form of the linear aging model, the model is extended to other stationary aging processes, such as the nonhomogeneous Poisson process, that satisfy the condition 


$$
\sum_{n=0}^{\infty} \mathrm{nP}_{n}(\mathrm{t})=\mathrm{Q}(\mathrm{t})
$$

where $Q(t)$ is the expected number of deteriorations that will occur to age $t$. In this case, the component failure rate becomes

$$
\bar{\lambda}(\mathrm{t})=\mathrm{k} \overline{\mathrm{x}} \mathrm{Q}(\mathrm{t})
$$

The overall aging failure rate of the component is the sum of the individual aging mechanism failure rates. For $k$ different aging mechanisms acting on the component, each with failure rate $\lambda_{i}\left(t_{i}\right)$, where $t_{i}$ is the age with respect to the $i$ th aging mechanism, the total aging failure rate, $\lambda$, would be

$$
\lambda_{\mathrm{A}}=\sum_{\mathrm{i}=0}^{\mathrm{k}} \lambda_{\mathrm{i}}\left(\mathrm{t}_{\mathrm{i}}\right) .
$$

If all the aging mechanisms have the same associated age, say $t$, then the total aging failure rate, $\lambda$, would be

$$
\lambda_{A}=\sum_{i=0}^{k} \lambda_{i}(t)
$$

The total component failure rate is then the sum of the contribution from nonaging, random failure causes and the aging failure causes, i.e.:

$$
\lambda_{\mathrm{T}}=\lambda_{0}+\lambda_{\mathrm{A}}=\lambda_{0}+\alpha \mathrm{t}
$$

where $\alpha$ is a constant.

We summarize the major assumptions used in the justification of the linear aging model in the list of comments below.

(1) The component failure rate is directly proportional to the specific amount of deterioration, $D$, that the component has experienced from a specific aging mechanism up to age t:

$$
\lambda(\mathrm{t})=\mathrm{kD} \text {. }
$$

(2) The amount of deterioration, D, is assumed to be random in both the times at which the deteriorations occur and the severity of damage incurred by the component each time a deterioration occurs.

(3) The occurrence of deteriorations is described by a Poisson process with a constant occurrence rate, $r$, i.e.,

$$
P_{n}(t)=\frac{(r t)^{n} e^{-r t}}{n !} \quad n=0,1, \ldots
$$

and the severity of deterioration increments at each occurrence are independent and identically distributed with a distribution $f(x)$. 


\subsubsection{Discussion}

As discussed in Sec. 2.4.1, the failure rate of a component is defined as the probability that the component will fail in the time increment $[t, t+d t]$, given that it has survived up to time $t$. In terms of a cumulative damage process, this can be interpreted as the probability that the component suffers an increment of deterioration in the time increment $[t, t+d t]$ large enough that, when added to the cumulative damage already incurred, causes the component to fail, given that it has survived up to time $t$. This probability requires a failure criterion, such as a threshold value for the total damage. Such a criterion does not appear in Eq. (2.45). As will be demonstrated later, when such a failure criterion is established, the assumption that the damage is accumulated linearly in time does not necessarily lead to a failure rate that is also linear in time.

The assumption that the damage increments occur according to a homogeneous Poisson process combined with the assumption that the damage increments are independent and identically distributed implies that the expected damage accumulates linearly in time. This would not be true if the damage increments occurred according to some other process, such as a nonhomogeneous Poisson process, or if the damage increments were not independent and identically distributed, e.g., if each damage increment depended on the previous history of damage accumulation. The assumption that the damage increments are independent and identically distributed does not apply in general to all degradation mechanisms, although it can be a useful approximation under specific conditions.

Although the linearly increasing failure rate model may not be applicable for all degradation mechanisms, it may well be a convenient approximation for many situations. A general drawback of the linearly increasing failure rate model, equally shared by the other reliability distributions that have been discussed, is that it essentially represents a parametric approximation made at a relatively high level of failure processes and mechanisms that usually have a rather complex physical foundation. Thus, although a particular parametric reliability distribution may fit the available failure data adequately, it will nevertheless always constitute a drastic simplification when examined from the point of view of such underlying physical phenomena. The obvious danger under these conditions is that a careless or superficial choice and application of parametric reliability models may obscure the understanding of the role played by important physical processes and parameters in the aging of important plant components. Thus, from this perspective, it becomes important to develop a degree of understanding of the correspondence, or lack thereof, that may exist between physically based models of aging processes and parametric reliability models such as those that are discussed in this section.

\subsection{Reliability Physics}

Reliability models and methods based on physical principles are presented and discussed in this section. The first part of this section is an overview of the important degradation mechanisms affecting NPP SSCs. The remainder of this section describes and discusses reliability methods and models associated with three of the most important degradation mechanisms, namely, fatigue, irradiation embrittlement, and stress-corrosion cracking. The primary aim of this section is to investigate whether the linear aging reliability model is an accurate representation of these important degradation mechanisms, and if it is not, whether there exists a more restricted set of conditions under which it would nevertheless constitute a reasonable approximation.

\subsubsection{Overview of Aging Mechanisms Affecting NPP SSCs}

In Shah et al. (1987), the components that are critical to plant safety during normal operation, off-normal situations, design-basis accidents, or severe accidents are identified and prioritized according to their role in preventing the release of fission products to the public. The most important components and mechanisms will be discussed here. Table 2-1 shows which aging mechanisms might be 
Table 2-1 Main PWR components and their degradation mechanisms

\begin{tabular}{|c|c|c|c|c|c|}
\hline & $\begin{array}{c}\text { Neutron } \\
\text { Embrittlement }\end{array}$ & $\begin{array}{c}\text { Time-Dependent } \\
\text { Relaxation } \\
\text { (Creep) }\end{array}$ & $\begin{array}{c}\text { Hydrogen } \\
\text { Embrittlement }\end{array}$ & $\begin{array}{l}\text { Stress } \\
\text { Corrosion } \\
\text { Cracking } \\
\end{array}$ & $\begin{array}{c}\text { Low-Cycle } \\
\text { Fatigue and } \\
\text { Fatigue/Creep }\end{array}$ \\
\hline $\begin{array}{l}\text { Reactor } \\
\text { Pressure Vessel } \\
\text { (RPV) }\end{array}$ & $\bar{X}$ & & & & \\
\hline $\begin{array}{l}\text { Containment } \\
\text { and Basement }\end{array}$ & & $x$ & $\bar{x}$ & $X$ & \\
\hline $\begin{array}{l}\text { Reactor } \\
\text { Coolant Piping } \\
\text { (RCP) }\end{array}$ & & $\bar{x}$ & & & $\bar{X}$ \\
\hline $\begin{array}{l}\text { Steam } \\
\text { Generators }\end{array}$ & & & & $\bar{X}$ & $\bar{x}$ \\
\hline $\begin{array}{l}\text { Reactor } \\
\text { Coolant Pump }\end{array}$ & & & & & \\
\hline Pressurizer & & & & & $X$ \\
\hline $\begin{array}{l}\text { Control Rod } \\
\text { Drive } \\
\text { Mechanism } \\
\text { (CRDM) }\end{array}$ & & & & & \\
\hline $\begin{array}{l}\text { Cables and } \\
\text { Connectors }\end{array}$ & & & & & \\
\hline $\begin{array}{l}\text { Emergency } \\
\text { Diesel } \\
\text { Generators }\end{array}$ & & & & & \\
\hline RPV Internals & 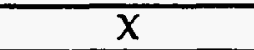 & & & $X$ & \\
\hline $\begin{array}{l}\text { RPV Supports } \\
\text { and Biological } \\
\text { Shields }\end{array}$ & $\bar{x}$ & & & & \\
\hline
\end{tabular}


Table 2-1 Main PWR components and their degradation mechanisms (Cont.)

\begin{tabular}{|c|c|c|c|c|c|}
\hline & $\begin{array}{c}\text { High-Cycle } \\
\text { and Thermal } \\
\text { Fatigue }\end{array}$ & $\begin{array}{l}\text { Corrosion } \\
\text { Fatigue }\end{array}$ & $\begin{array}{c}\text { Thermal } \\
\text { Embrittlement }\end{array}$ & $\begin{array}{c}\text { Mechanical } \\
\text { Wear and } \\
\text { Fretting } \\
\end{array}$ & $\begin{array}{c}\text { Corrosion and } \\
\text { Corrosion- } \\
\text { Erosion }\end{array}$ \\
\hline $\begin{array}{l}\text { Reactor } \\
\text { Pressures } \\
\text { Vessel (RPV) }\end{array}$ & $x$ & $x$ & & & \\
\hline $\begin{array}{l}\text { Containment } \\
\text { and Basement }\end{array}$ & & & & & $\bar{X}$ \\
\hline $\begin{array}{l}\text { Reactor } \\
\text { Coolant Piping } \\
\text { (RCP) }\end{array}$ & $\bar{X}$ & & $\bar{X}$ & & \\
\hline $\begin{array}{l}\text { Steam } \\
\text { Generators }\end{array}$ & $\bar{x}$ & $\bar{x}$ & & $\bar{x}$ & \\
\hline $\begin{array}{l}\text { Reactor } \\
\text { Coolant Pump }\end{array}$ & & $\bar{x}$ & $\bar{x}$ & & \\
\hline Pressurizer & $\bar{x}$ & & $\bar{x}$ & & $\bar{x}$ \\
\hline $\begin{array}{l}\text { Control Rod } \\
\text { Drive } \\
\text { Mechanism } \\
\text { (CRDM) }\end{array}$ & & & $\bar{x}$ & $\bar{x}$ & \\
\hline $\begin{array}{l}\text { Cables and } \\
\text { Connectors }\end{array}$ & & & $\bar{x}$ & & $\bar{x}$ \\
\hline $\begin{array}{l}\text { Emergency } \\
\text { Diesel } \\
\text { Generators }\end{array}$ & $\bar{X}$ & & & & \\
\hline RPV Internals & $x$ & & & & \\
\hline $\begin{array}{l}\text { RPV Supports } \\
\text { and Biological } \\
\text { Shields }\end{array}$ & & & & & $\bar{x}$ \\
\hline
\end{tabular}


important contributors to degradation for major pressurized water reactor (PWR) and boiling water reactor (BWR) components.

\subsubsection{Fatigue}

Fatigue is by far the most common mode of failure for metals. Fatigue is a cumulative damage process that results from a component be subjected to a cyclic load of sufficient intensity. In fatigue failure, cyclic stresses cause the initiation of cracks (if none are already present), which propagate through the material until the stress on the remaining resisting area is sufficient to cause an abrupt failure.

Fatigue is the primary degradation mechanism associated with piping. The main fatigue mechanism affecting PWR primary coolant piping is low-cycle fatigue caused by a combination of pressure and transient thermal stresses. The points of stress concentration in the system are the areas of most concern. These sites include the nozzles welded into the primary coolant piping and the terminal ends of the piping. So far as it is known, there have been no failures or even discovered cracks in existing PWR reactor coolant piping.

The primary degradation mechanism affecting BWR reactor pressure vessels is fatigue. Three of the highest ranking degradation sites have been eliminated in the newer plants by design changes, and they have been repaired in most of the older plants. These include austenitic stainless-steel safe ends that have been sensitized by post-weld heat treatment, crevice areas where thermal sleeves are attached to Iconel or stainless-steel safe ends, and austenitic cladding on the inside corners and surrounding regions of the nozzles. Another important potential degradation site is the outer end of the nozzle forgings and the attached safe ends. The most severely affected nozzle of this type is the feedwater nozzle. The best example of problems caused by flow through this nozzle is the Millstone-1 plant, where nozzle corner cracking was found. In general, all locations of dissimilar metal welds will be most sensitive to thermal cycling. The most important areas in this regard are the nozzle safe-end attachments and the attachment welds to the pressure boundary for the core support structure.

Most of the fatigue problems in PWR pressure vessels involve geometric discontinuities at sites such as the closure head studs, outlet and inlet nozzles and instrumentation nozzles, and CRDM housings.

PWR reactor coolant pump bodies are subject to thermal and mechanical fatigue damage caused by system operating transients and pump vibrations. The RCP body fatigue life is usually very conservative and is not considered to be an issue for license renewal.

The PWR pressurizer spray and surge nozzles are subject to high-fatigue usage induced by changes in reactor power and plant heatup and cooldown cycles. The cylindrical portion of the pressurizer vessel can undergo fatigue damage caused by heatups and cooldowns, variations in water level caused by insurges and outsurges, and the effects of the subcooled spray water contacting the upper shell. The failure mode for the pressurizer vessel probably would be a leak caused by ductile fracture of the metal rather than a catastrophic break. Failure of the spray head would be gradual and would degrade the ability of the pressurizer to control pressure surges because the spray head would not be able to spray uniformly.

Fatigue is the main degradation mechanism for the pressurizer surge line. Design-basis thermal transients cause both high-and low-cycle fatigue damage. High-cycle fatigue is caused by small temperature changes during normal operation and results in very little damage. Low-cycle fatigue is caused by a combination of transient pressure and thermal stresses and is much more damaging. Stratified flows cause both high- and low-cycle fatigue damage to the horizontal portion of the surge and spray lines. The wavy character of the mixing layer causes rapid changes in the temperature of the inside surface of the piping, and fluctuations in the stratified flow cause low-cycle 
fatigue damage because of changes in the through-wall bending stresses. Potential failure modes of the surge line include fatigue-induced crack initiation and propagation leading to a through-wall leak. A degraded surge line may rupture during an earthquake.

Heatup, cooldown, and pressure testing cause thermal and mechanical low-cycle fatigue at several sites in BWR metal containments. These include sites with geometric discontinuities that act as stress concentrators and sites with adjacent materials with different thermal expansion coefficients.

\subsubsection{Irradiation Embrittlement}

Irradiation embrittlement is the major degradation mechanism associated with PWR pressure vessel aging. It is less important for BWR pressure vessels because of the lower fluence experienced in the BWR vessel environment.

The effect of neutrons bombarding the inside wall of the reactor vessel is a serious issue for maintaining vessel integrity. As a result of irradiation exposure, the ferritic steel strength increases with an attendant decrease in ductility. In terms of the Charpy V-notch (CVN) energy properties, which correspond to fracture toughness properties, the ductile-to brittle-transition temperature increases and the upper shelf-energy decreases. In the Charpy V-notch test, the energy required to break a sample is measured by breaking a notched sample element with a swinging pendulum and measuring how much energy was absorbed (Fig. 2.14). Below the ductile-to-brittle transition temperature, material behaves in a brittle manner; above the transition temperature it behaves in a ductile manner. The upper shelf energy corresponds to the fracture energy associated with $100 \%$ ductile tearing.

The main concern associated with irradiation embrittlement is the possibility of failure of the pressure vessel as a result of brittle fracture (or ductile tearing) caused by a pressurized thermal shock (PTS) event. In such an event, the emergency core cooling system (ECCS) injects cool water into the vessel, and the resulting thermal stresses, in addition to the primary stresses because of the system pressure, will have the potential to initiate fracture at a pre-existing defect at the vessel inner surface. If the crack were to propagate through the entire vessel thickness, a through-wall leak or even catastrophic failure could occur.

The major variables controlling irradiation embrittlement are the copper and nickel content of the steel and the total neutron fluence. Other variables believed to be important include the irradiation temperature, the neutron spectrum and flux, the phosphorus content, the thermomechanical history, the concentrations of some other impurities, and minor alloying elements.

Embrittlement normally is monitored by testing samples of base, heat-affected-zone (HAZ) and weld materials in the form of CVN and tension specimens that have been irradiated in surveillance capsules near or adjacent to the vessel wall. This represents an effort to come as close as possible to simulating actual conditions experienced by the vessel itself. Appendix $\mathrm{H}$ to 10 CFR 50 requires that the samples be selected from the most limiting material. There are usually several surveillance capsules that are tested at specified intervals over the life of a vessel. Neutron flux in the surveillance capsules is greater than that in the actual vessel by a lead factor that is intended to provide an early warning of unacceptable levels of property degradation. Each CVN specimen provides an energytemperature pair. Typically, about 12 specimens are CVN tested at various temperatures to define the energy-temperature curve, and this curve provides two measures of embrittlement. The change in the upper shelf energy (USE) and the shift in the transition temperature at the 41-J level.

In practice, embrittlement is monitored by changes in notch ductility parameters as measured in $\mathrm{CVN}$ impact testing. The CVN transition temperature increase referenced at the 41-J energy level, as well as reductions in the CVN USE, are used to characterize the degree of embrittlement in pressure vessel surveillance programs as required by the Code of Federal Regulations. Data from reactor vessel surveillance programs have been compiled in an EPRI report (Oldfield, 1986), which contains the 


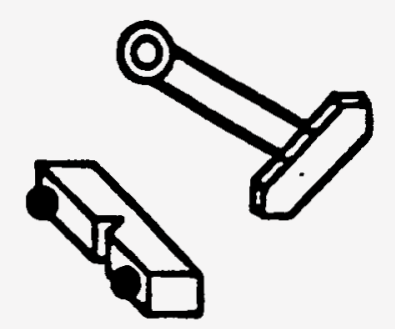

Charpy Test

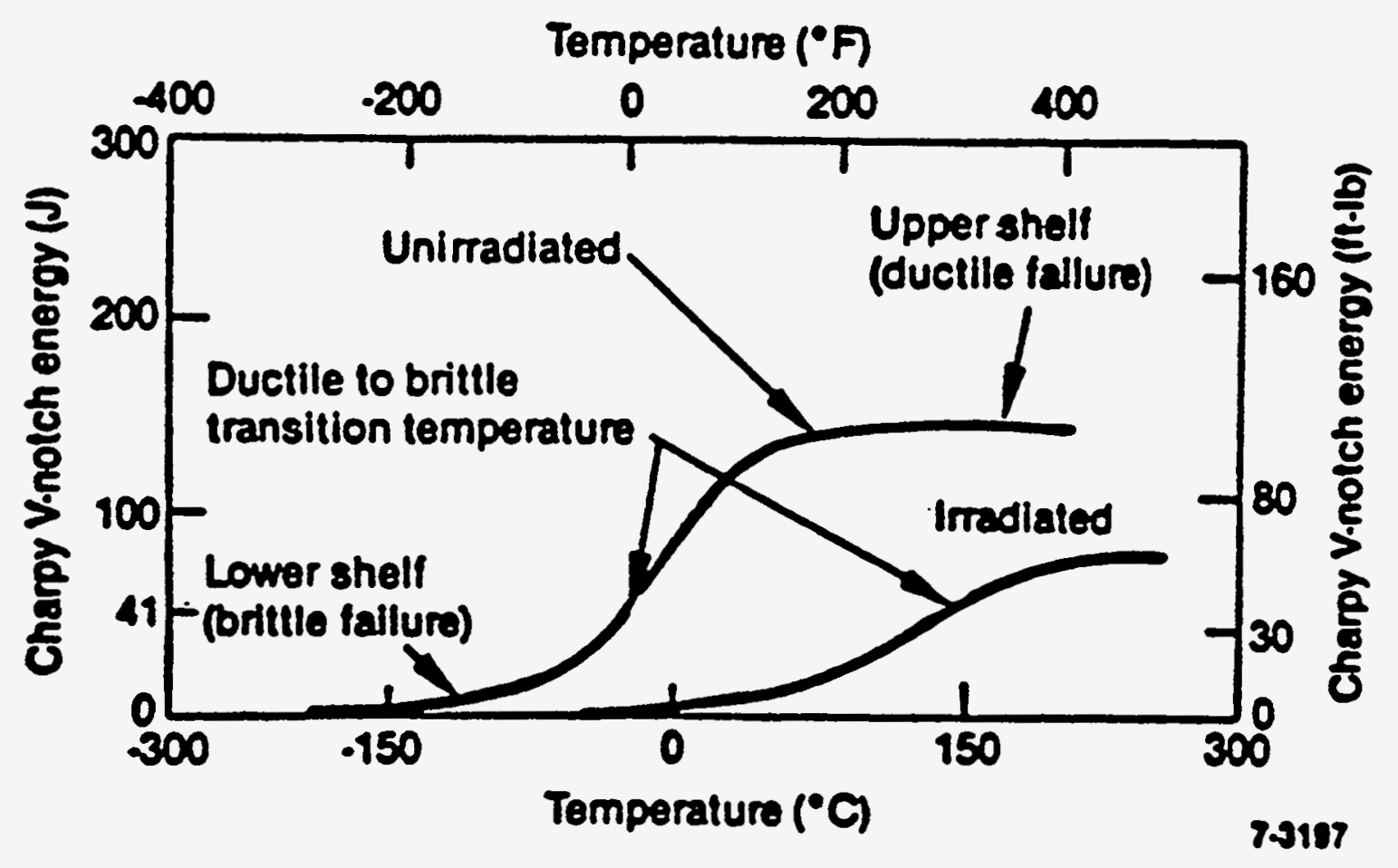

Figure 2.14 Effect of irradiation on the Charpy energy for a nuclear pressure vessel steel 
measured results from 57 reactors and 116 capsules. The data from the CVN tests and information on material chemistry and accumulated fluence have been included in a data base for development of improved radiation damage prediction methods. Also, the Power Reactor Embrittlement Data Base compiled at Oak Ridge National Laboratory (Stallman and Kam, 1991) lists the test results of 117 base materials (plates and forgings, 85 welds, and 88 heat-affected-zone materials that were irradiated in 241 capsules of 82 reactors. A variety of correlations have been proposed to relate CVN measurements directly to toughness, but their general validity has not been demonstrated yet (Odette and Griesbach, 1987).

Other components for which irradiation embrittlement is an important degradation mechanism include the reactor pressure vessel internals and the reactor pressure vessel supports.

\subsubsection{Stress-Corrosion Cracking}

Stress-corrosion cracking (SCC) is a form of environmentally assisted cracking (EAC) and occurs when a component is under stress in a corrosive environment. The observed crack propagation is the result of the combined and synergistic interaction of mechanical stress and corrosion reactions. Many different mechanisms have been proposed to explain the synergistic stress-corrosion interactions that occur at the crack tip, and there may be more than one process that causes SCC. Most proposed SCC mechanisms assume that breaking of the interatomic bonds of the crack tip occurs either by chemical solution and dissolution or by mechanical failure, were normal fracture processes are assumed to be stimulated or induced by interactions between the material and the environment.

\section{Stress Corrosion Cracking Mechanism (SCC)}

Surface defects may pre-exist or could be nucleated under the influence of fluctuating mechanical or thermal stresses. Surface cracks, if nucleated under fatigue loading, are generally a result of the propagation of Persistent Slip Bonds (PSBs) to the surface forming extrusions and intrusions in adjacent locations. These surface locations, which tend to be crack initiation points, have higher Gibb's free energy, as compared to other surface spots. Chemical corrosive reactions are therefore enhanced or intrusion/extrusion planes.

Crack propagation is favored once the stress intensity factor exceeds a critical value, that is, mainly determined by the surface energy on the plane of crack propagation, and the energy dissipated in the plastic zone ahead of the crack. The high chemical corrosion rate at the crack tip is driven by the fast diffusion rate of embrittling impurities towards the crack tip. The surface energy is thereby reduced and the critical stress intensity factor decreases. This is an unstable process, possibly limited by the corrosion and/or the diffusion rate of impurities.

Stress-corrosion cracking is a common problem for PWR steam generator tubes. Increasingly, tubes have been removed from service as a result of primary-to-secondary leaks at points of high stress concentration because of intergranular stress-corrosion cracking (IGSCC). High stress concentration regions include the Row $1 \mathrm{U}$-bends, the rolled transition regions in the tube sheet, and dented intersections. SCC on the outside surface of the tubing has become an important problem.

SCC is also an important degradation mechanism in BWR recirculation piping, where failures (leaks) have been primarily a result of IGSCC. Although the conditions for IGSCC exist in the heataffected zones of most BWR recirculation piping welds, only a small percentage of these welds have cracked.

IGSCC in the HAZs of the PWR pressurizer nozzle spray heads is possible, but there is no evidence of this to date. High residual stress in the heater sheath walls can promote SCC. The 
stainless-steel bellows in BWR metal containments are susceptible to intergranular and transgranular SCC. However, this has not been observed.

\subsubsection{Corrosion and Other Mechanisms}

Many different types of corrosion affect NPP components. They include uniform attack, galvanic corrosion, crevice corrosion and pitting, differential aeration, and microbially influenced corrosion. Uniform attack is characterized by the general corrosion of the exposed or deteriorated surface, and as a result, the metal becomes thinner. Galvanic corrosion occurs between two dissimilar metals characterized by differing corrosion potentials when they are immersed and electrically connected in a corrosive environment. Pitting and crevice corrosion are forms of local corrosion that occur on metal surfaces exposed to locally stagnant or slow moving liquids. Microbially influenced corrosion is the deterioration of a metal by a corrosion process occurring directly or indirectly as a result of metabolic activities of microorganisms.

BWR metal containments are constructed of carbon steel, and because of the containments and reactor building environments, the potential for corrosion is high. This is particularly true if the metal surface is uncoated or if the coating has deteriorated. The carbon steel area near dissimilar welds between the stainless-steel bellows and the carbon-steel pipes in the vent and penetration lines are potential sites for galvanic corrosion. In Mark III metal containments, the submerged portion of the containment wall is clad stainless steel. The area on the unclad wall surface near the stainless steel clad is susceptible to galvanic corrosion if the coating has deteriorated, and sites of local failures in the coating are susceptible to pitting. Potential sites for crevice corrosion include the interface of the concrete and metal shell near the embedment if a crack or gap is present, cracks or crevices formed between the mating surfaces of metal assemblies, and the gap between the drywell wall and the shield wall in Mark I and Mark II containments. Local corrosion caused by differential aeration is caused by a gradient in the amount of dissolved oxygen near the water line. The submerged metal surfaces of Mark III containments are clad with stainless steel and therefore are not susceptible to this type of corrosion. Two sites on BWR containments may provide the environment necessary to support microorganisms: the water in the suppression pools and the sand pockets near the base of the Mark I drywells.

In BWR recirculation piping, crevice corrosion potentially could occur over time at locations such as shaft sleeves and the underside of socket welds if a corrosive environment and a difference in oxygen concentration existed. To date, crevice corrosion has not been reported as a problem in BWR recirculation piping.

Another form of corrosion, microbiological-induced corrosion, occurs in PWR concrete containments. The post-tensioning system anchors and tendon wires are particularly susceptible to this corrosion mechanism.

Thermal embrittlement is a degradation mechanism that affects the PWR reactor coolant pump bodies and the PWR primary piping. Thermal embrittlement of the base metal of the RCP bodies results in a slow loss of fracture toughness over extended periods of time and is influenced by the coolant temperature and the time of exposure at temperature, as well as by the chemical composition of the base metal. If thermal embrittlement ever becomes a problem (considered unlikely), unstable ductile tearing of the pump body during a design transient could be a potential failure mode. With respect to thermal embrittlement of PWR primary piping, the concern is that the ductile tearing resistance of the material is reduced by thermal aging so that the potential for ductile fracture becomes a problem.

The impingement of solid particles on steam generator tubes under operating conditions can cause mechanical damage known as erosion-corrosion. Solid particles are entrained in the bulk flow and impact against the tube, promoting erosion. In a corrosive environment, the erosion process may first remove a protective film from the tube, thus making it susceptible to corrosion. Material loss occurs, either directly or by accelerated corrosion of the tube surface. Erosion-corrosion has occurred in 
once-through steam generators around the fourteenth support plate and has caused more than a $40 \%$ wall-thickness reduction in some steam generators. In another incident, erosion-corrosion inspections at the Susquehanna Steam Electric Station identified an unexpected high rate of erosion-corrosion in certain main feedwater piping inside containment (USNRC, 1992).

Mechanical wear and fretting are additional aging mechanisms experienced by steam generator tubes. Tube fretting and wear are caused by vibration, which vibration can be induced by fluid crossflow perpendicular to the tubes or parallel flow along the tubes. Fretting regions are sensitive to fatigue cracks. Under fretting conditions, fatigue cracks can initiate close to the material surface at very low stresses, well below the fatigue limit of nonfretted tube material. If the vibration cyclic stresses are sufficient to cause these cracks to grow, early failure of the tube can occur. Fretting has caused extensive damage at the antivibration bars in several steam generators, which has been blamed on poor design of the antivibration bars.

\subsubsection{Stress-Strength Interference Models}

In general, estimation of the reliability of engineering structures and components based on physical phenomena (rather than estimation of distribution parameters from lifetime data) involves comparison of the strength of the component to the applied stresses (loads). Failure occurs when the applied load is greater than the component strength. Such models, commonly called stress-strength interference (or, reliability physics) models, involve determining the failure probability of the component for a single application of stress and then combining that probability with a model for the times at which stresses occur. If the strength, or resistance, of a component can be characterized as a random variable $X$ having a density function $f(x)$, and the applied stress is characterized as a random variable $Y$ with a density function $g(y)$, then the reliability for a single load application is the probability that $X>Y$, or

$$
R=P(X>Y)=\int_{0}^{\infty}[1-F(y)] g(y) d y
$$

where

$$
F(y)=\int_{0}^{y} f(x) d x
$$

Figure 2.15 illustrates the concept of stress-strength interference and how it relates to the stress and strength density functions.

How the stresses occur in time can be either cyclic (known time intervals) or random, and the stress amplitudes can be either constant or varying. Random occurrences are usually, but not always, modeled as a homogeneous Poisson process. In the case of Poisson stress occurrences (with a rate $\alpha$ ), random-independent stress and random-fixed strength, the reliability is given by

$$
R(t)=\sum_{i=0}^{\infty} \frac{e^{-\alpha t}(\alpha t)^{i}}{i !} \int_{0}^{\infty} g(y)[1-F(y)]^{i} d y
$$

Stresses and strengths may vary during long operating intervals in relation to the passage of time or the number and/or severity of stress applications. Aging denotes the decrease in strength as a function of time, cyclic damage denotes the decrease in strength as a function of the number of stress cycles, and cumulative damage denotes the case in which the decrease in strength is determined by both the number and severity of stresses. Thorough discussions on time-dependent stress-strength interference models can be found in Kapur and Lamberson (1977), Shaw et al. (1980, 1981), and Shen (1988). 


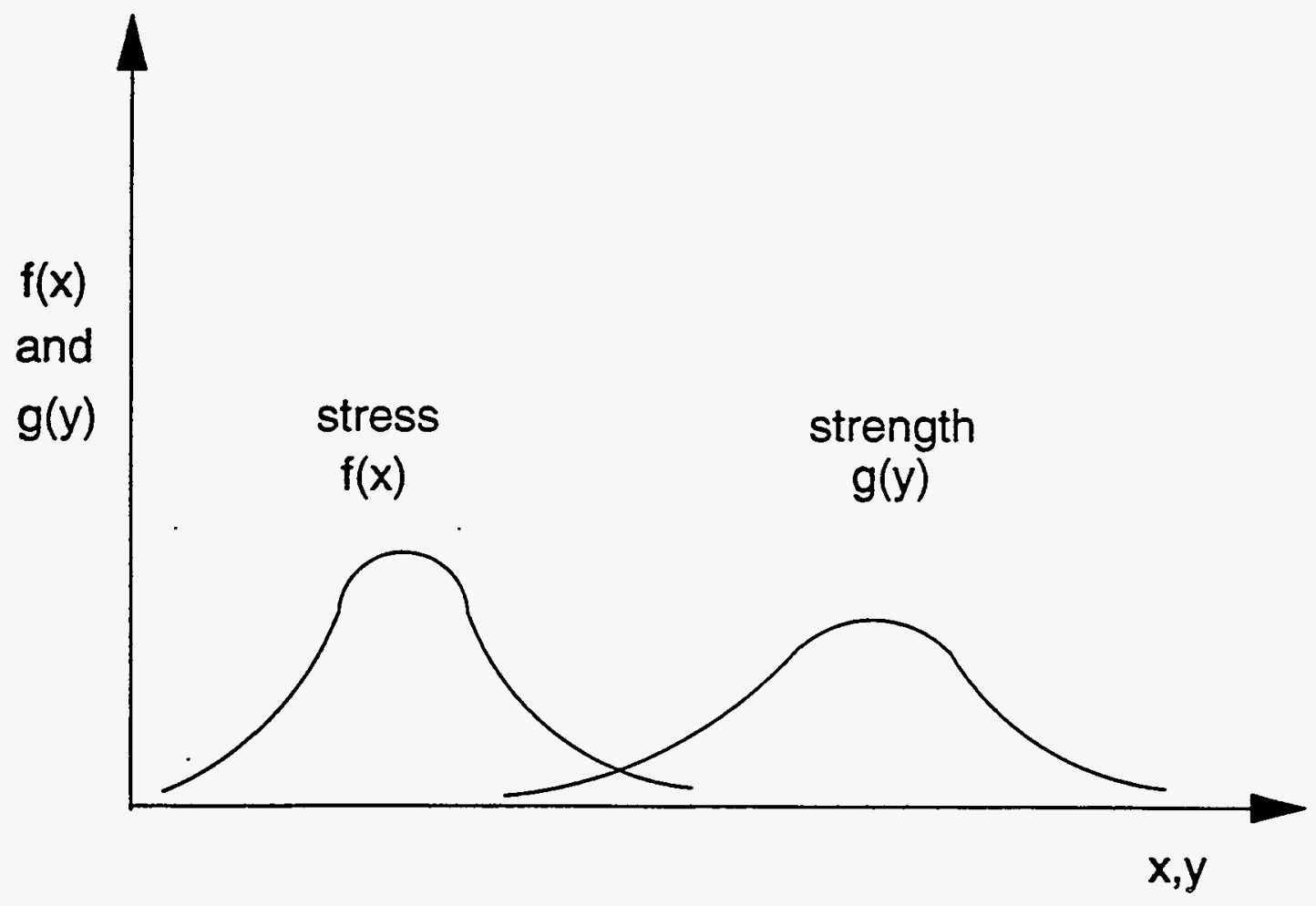

Figure 2.15 Stress-strength interference 


\subsubsection{Fatigue}

As discussed in Sec. 2.4.1.2, fatigue is an important deterioration process for many components, including the reactor pressure vessel, piping BWR metal containments, RCP pump casings, reactor internals, and many others.

Under cyclic stress conditions, dislocation dipole alignments result in localized plastic deformations. Microscopic cracks are created that then grow and join to produce major cracks. Crack initiation and crack growth commonly are regarded as the basic cause of fatigue damage accumulation and ultimate fatigue failure. In the following, the basic methods of deterministic fatigue analysis will be reviewed. These models provide the basis for the stochastic models that will be discussed in the next section.

\subsubsection{General Deterministic Models}

\section{Cycles To Failure Models}

These models provide an estimate of the number of stress cycles to failure of the material. In design, they are used to predict the fatigue usage a component will experience over its design lifetime. The fatigue usage is the ratio of the stress cycles the component is expected to experience over its lifetime to the predicted cycles-to-failure.

\section{S-N Curves}

\section{Model Objectives:}

S-N curves are used to predict the number of cycles to failure at a single stress level.

\section{Model Formulation:}

S- $\mathrm{N}$ curves conveniently display basic fatigue data on a plot of cyclic stress level vs number of cycles to failure. Analytical representation of S-N curves commonly is given in the form

$$
N S^{b}=k
$$

where $\mathrm{b}$ and $\mathrm{k}$ are material parameters estimated from test data obtained using identical specimens.

Model Assumptions:

S-N curves assume cycles at a single stress level.

Discussion:

The S-N approach, though widely used in design applications, does not deal with any of the physical phenomena within the material. It does not separate the crack initiation phase from the crack propagation phase, and only the total life to fracture is considered. 


\section{The Palmgren-Miner Rule}

\section{Model Objectives:}

The Palmgren-Miner rule (or Miner's rule) is a linear damage accumulation rule used to predict the cycles to failure under variable amplitude loading.

Model Formulation:

The Palmgren-Miner rule asserts that the damage fraction, $\Delta_{i}$, at any stress level $S_{i}$ is linearly proportional to the ratio of $n_{i}$, the number of cycles of operation under this stress amplitude, to $N_{i}$, the total number of cycles that would produce a failure at that stress level; i.e.,

$$
\Delta_{i}=\frac{n_{i}}{N_{i}},
$$

where $n_{i} \leq N_{i}$. If the stress amplitude is changed, a new partial damage is calculated for this new amplitude level, where the appropriate $N_{i}$ is found from the S-N curve. The total accumulated damage then is given by

$$
D=\sum_{i} \Delta_{i}=\sum_{i} \frac{n_{i}}{N_{i}},
$$

and failure occurs when $D \geq 1$.

\section{Model Assumptions:}

The Palmgren-Miner rule assumes that damage is accumulated linearly. Also, it assumes that damage caused by a stress cycle is independent of where it occurs in the load history. Another assumption is that failure occurs when $D \geq 1$, although experiments have shown that typical values of $\mathrm{D}$ at failure range from 0.5 to 2 .

\section{Discussion:}

In the Palmgren-Miner rule, the only material characteristic considered is the number of cycles to failure under constant amplitude loading. While the absolute validity of the Palmgren-Miner rule is in question, its comparative usefulness as a simple criterion for comparing different designs is of considerable value. Also, in Miner's rule does not consider the effect of environment on fatigue life, which is important for LWR pressure boundary components.

There are several other damage accumulation rules, similar to Miner's Rule, but the damage accumulation is a nonlinear function of the fractional damage within each cycle range.

\section{Fracture Mechanics}

Fracture mechanics is divided into Linear Elastic Fracture Mechanics (LEFM) and ElasticPlastic Fracture Mechanics (EPFM). Materials with relatively low fracture resistance that fall below their so-called plastic collapse strength can be analyzed on the basis of elastic concept through the use of LEFM. For other materials, the use of EPFM is often necessary. 


\section{Linear Elastic Fracture Mechanics}

Crack propagation laws are models that relate the crack growth rate to the level of cyclic stress. Using the methods of linear elastic theory, the stresses at any point in the structural component can be characterized in principle. In general, they are complex and cannot be given in an analytical form. However, for stresses near the crack tip and using assumptions of either plane stress or plane strain, an asymptotic solution of stress field yields a formula for the stress components $\sigma_{\mathrm{ij}}$ that is relatively simple and is given by

$$
\sigma_{i j}=\frac{K}{\sqrt{2 \pi r}} f_{i j}(\theta)
$$

where $r$ and $\theta$ are polar coordinates with respect to the crack tip and $K$ is the stress intensity factor. In general, the stress intensity factor has the form

$$
\mathrm{K}=\mathrm{BS} \sqrt{\pi \mathrm{a}} \text {, }
$$

where $a$ is the crack length, $S$ is the far-field stress resulting from the applied load, and $B=B(a)$ is a factor that accounts for geometry. For any crack in any practical problem only the factor $B$ needs to be derived. For many geometries, B has been calculated already and can be found in handbooks.

Values of $\mathrm{K}$ for various loadings and geometrical configurations can be evaluated from elasticity theory. The most common reference value of $\mathrm{K}$ is for a two-dimensional center crack of length $2 a$ imbedded in an infinite sheet and subjected to a uniform tensile stress $S$. In this case, $B=1$ and

$$
\mathrm{K}=\mathrm{S} \sqrt{\pi \mathrm{a}}
$$

Fracture will occur when the stresses at the crack tip become too high for the material to bear. Since the stress intensity factor, $K$, determines the entire crack tip stress field, when the stress intensity reaches a critical value $K_{c}$, unstable fracture occurs. This critical value of $K$ is known as the fracture toughness of the material. In situations in which the toughness is very high or the crack is very small, failure by plastic collapse may occur before $\mathrm{K}$ reaches $\mathrm{KIC}$.

\section{Elastic-Plastic Fracture Mechanics}

If fracture is accompanied by considerable plastic deformation EPFM is used. The fracture parameter used is often referred to as the "J-integral." J is simply the strain energy release rate, and it can be derived from a conservation of energy criterion. In EPFM fracture occurs when

$$
\mathrm{J}=\mathrm{J} \mathrm{R}
$$

where $J_{R}$ is the fracture energy, which represent the materials fracture resistance.

EPFM deals with high toughness materials. For such materials, the fracture energy, $\mathrm{J}_{\mathrm{R}}$, tends to increase during the fracture process, so that fracture will be slow and stable initially, until at some point an instability occurs causing the fracture to become fast and uncontrollable. Stable fracture is slow and will be stopped unless the stress is further increased.

Figure 2.16 shows the rising $J_{R}$-curve. Fracture occurs when $J=J_{R}$. At an existing crack, the curve for $\mathrm{J}$, which also increases with increasing crack length, can be drawn for a number of values of stress. At stress $\sigma_{a}$, the value of $J(a)$ is given by point A. Since $J<J_{R}$, fracture is not possible. If the stress is 
increased to $\sigma_{i}, J=J_{R}$ and fracture occurs. However, this fracture is stable and cannot proceed if the stress remains equal to $\sigma_{\mathrm{i}}$. For fracture to continue, the stress must be increased to $\sigma_{\mathrm{b}}$, which brings $\mathrm{J}$ to point $D$. If the stress reaches $\sigma_{\mathrm{fr}}$, the fracture becomes unstable.

From Fig. 2.16, the condition for instability is

$$
\begin{aligned}
& J=J_{R} \\
& \frac{d J}{d a}=\frac{d_{R}}{d a}
\end{aligned}
$$

which is valid if the stress does not drop once the fracture becomes unstable. In most cases, the difference between $\sigma_{\mathrm{i}}$ and $\sigma_{\mathrm{fr}}$ is small so that the criterion of Eq. (2.68) is adequate.

\section{Crack Propagation Laws}

\section{The Paris-Erdogan Equation}

\section{Model Objectives:}

The Paris-Erdogan equation is an empirical model used to calculate the fatigue crack growth rate under cyclic stresses. It is an empirical formula that relates the crack growth rate (per cycle) to the stress intensity factor range.

\section{Model Formulation:}

The Paris-Erdogan equation takes the form

$$
\frac{\mathrm{da}}{\mathrm{dN}}=\mathrm{C}(\Delta \mathrm{K})^{\mathrm{m}}
$$

where $\mathrm{da} / \mathrm{dN}$ is the crack growth rate, $\Delta \mathrm{K}$ is the stress intensity factor range $K_{\max }-K_{\min }$, and $C$ and $\mathrm{m}$ are material constants.

\section{Model Assumptions:}

The Paris-Erdogan law assumes that the crack growth rate depends only on the stress intensity factor range. It also assumes that the stress amplitude is constant and that it is small enough so that linear-elastic fracture mechanics (LEFM) is applicable and that the crack growth rate is independent of the previous load history. Failure occurs when the stress intensity factor exceeds the fracture toughness.

\section{Discussion:}

The Paris-Erdogan equation is an empirical formulation. The material parameters $\mathrm{C}$ and $\mathrm{m}$ depend on many factors, including material properties, environment, cycle frequency, temperature, etc., and their numerical values deviate from experiment to experiment.

Many other similar crack growth laws exist, many of which also include the stress ratio, $\mathrm{R}=\mathrm{S}_{\min } / \mathrm{S}_{\max }$. Also, there are models that treat nonlinear damage accumulation where crack growth retardation due to overloadings, and acceleration due to underloading are considered. An excellent 
review of the various cumulative damage rules and crack growth laws can be found in Sobczyk and Spencer (1992).

\subsubsection{Physical Fatigue Reliability Models and Comparison with the Linear Aging Model}

Fatigue is a cumulative damage process, and in general, the reliability of a structure or component subject to a cumulative damage process can be given by

$$
R(t)=\sum_{i=0}^{\infty} \frac{e^{-\alpha t}(\alpha t)^{i}}{i !} \int_{0}^{\infty}\left[\prod_{j=0}^{i} F\left(y_{j}\right)\right] g\left(y_{0}\right) d y_{0}
$$

where the stress applications are Poisson, $F(x)$ is the cumulative distribution of the stress magnitude, and $y_{j}$ is the strength at the $j$ th stress application given by

$$
\mathrm{y}_{\mathrm{j}}=\psi(\mathrm{j}) \mathrm{y}_{0},
$$

where $\psi(j)$ is a decreasing function of the stress history.

Because fatigue, in its many physical forms, is a major deterioration and aging process, we will derive reliability models for their effects on the failure rates of affected reactor components starting from the general formulation given by Eq. (2.71). In these models, knowledge of basic material statistical data and a statistical description of in-service loads are required to predict failure rates. The objective of the work presented here is to show how the component failure rates are related to the statistical material properties and loading conditions and to compare these results with the aging reliability model.

\section{Constant Stress Amplitude and Random Strength}

In general, fatigue samples are tested at a fixed stress amplitude (or fixed strain amplitude), and the test is conducted until the sample fails. In this regard, when the test is performed under a constant stress amplitude and the sample fails after a limited number of cycles, $\mathrm{N}$, the stress is termed the fatigue strength at the given life, N. Because the intrinsic strength of the material is distributed statistically around a mean value, $\mu_{\mathrm{S}}$, it also is expected that the lifetime, $N$, will be a random variable at a constant applied stress. This situation is illustrated in Fig. 2.16.

The relationship between the strength, $S$, and the lifetime, $N$, does not usually follow a simple power law but displays curvature on a $\log (\mathrm{N})$ plot. However, for simplicity of presentation, this relationship is represented here as a straight line on the $S$ vs $\log (N)$ diagram as shown in Fig. 2.17.

The strength-life relationship may now be put in the form

$$
S=S_{y}-\left[\frac{\ln \left(\frac{N}{N_{y}}\right)}{\ln \left(\frac{N_{e}}{N_{y}}\right)}\right]\left(S_{y}-S_{f}\right) .
$$

Typically for steels, $\mathrm{N}_{\mathrm{e}} \approx 10^{6}$ and $\mathrm{N}_{\mathrm{y}} \approx 10^{2}$. Equation (2.73) can be put in the simpler form: 


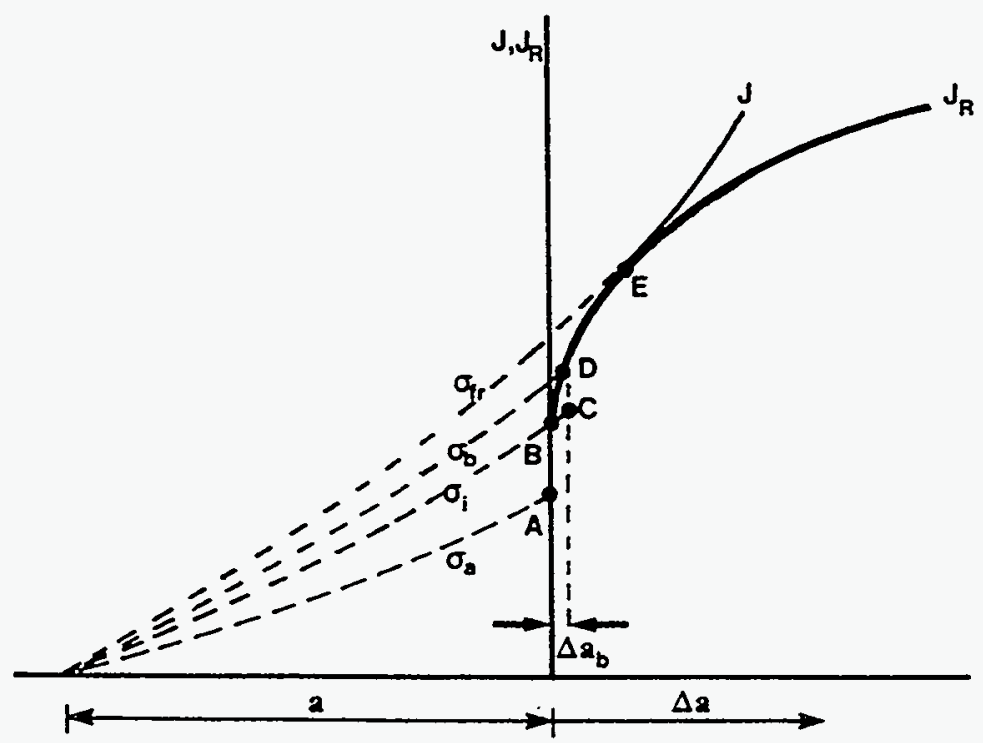

Figure 2.16. J-curves for different stresses and $J_{R}$-curve. 


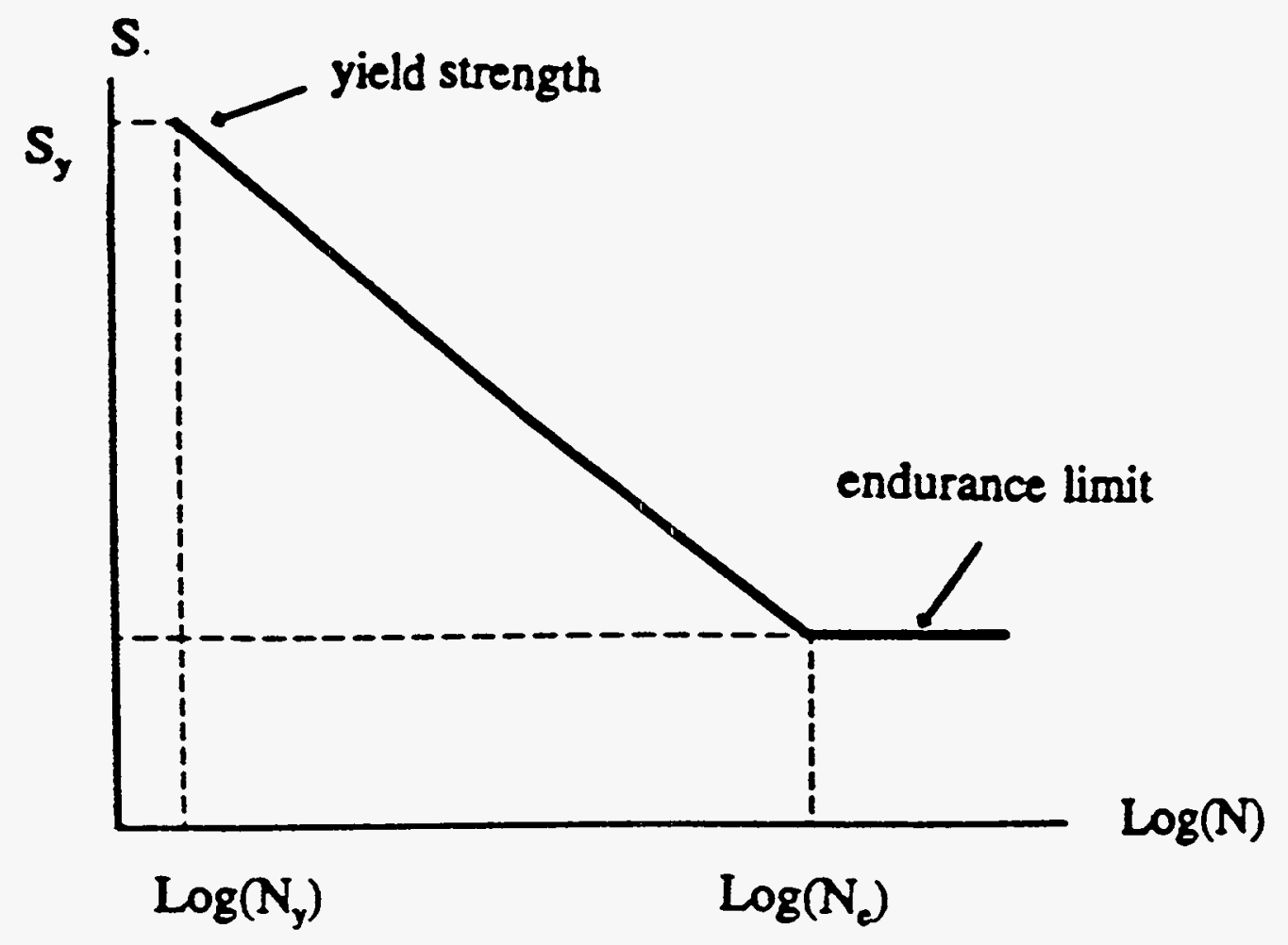

Figure 2.17 Approximate engineering strength-life relationship 


$$
S=a-b \ln N,
$$

where $\mathrm{a}$ and $\mathrm{b}$ are appropriate constant. Both Eqs. (2.73) and (2.74) are valid for $\mathrm{N}<\mathrm{N}_{e}$.

\section{Gaussian Strength Distribution at a Constant Stress Amplitude}

For most materials, it usually is assumed that the distribution of strength is typically Gaussian around a mean value, $\mu_{S}$, and with a standard deviation, $\sigma_{S}$. For a constant stress amplitude and at a fixed value of lifetime, $\mathrm{N}$, the pdf then is given by

$$
f(s)=\frac{1}{\sigma_{s} \sqrt{2 \pi}} \exp \left[-\frac{1}{2}\left(\frac{s-\mu_{s}}{\sigma_{s}}\right)^{2}\right]
$$

If $\mathrm{N}$ is the random lifetime value that corresponds to a random strength, $\mathrm{S}$, for a fixed-stress amplitude level, invoking the principle of probability conservation, we obtain

$$
F(S) d S=\bar{f}(N) d N
$$

where $\bar{f}(N)$ is the pdf for failure lifetime at a fixed stress amplitude level.

Using Eq. (2.74), Eq. (2.76) leads to

$$
f(N)=\left|f(S) \frac{d S}{d N}\right|=\frac{b}{\sigma_{N} N \sqrt{2 \pi}} \exp \left\{-\frac{1}{2}\left[\frac{b \ln \left(N / \mu_{N}\right)}{\sigma_{N}}\right]^{2}\right\},
$$

which is a lognormal pdf for the cycles to failure at a constant stress amplitude.

If we further assume that the occurrence of the stress level is at a constant frequency, $r$, the time-dependent failure rate is given by [using Eq. (2.26)]

$$
\lambda(\mathrm{t})=\frac{\frac{1}{\mathrm{t}} \exp \left[-\frac{1}{2}\left(\frac{\ln (\mathrm{t} / \overline{\mathrm{t}})}{\mathrm{M}}\right)^{2}\right]}{\int_{\mathrm{t}}^{\infty} \frac{1}{\mathrm{t}^{\prime}} \exp \left[-\frac{1}{2}\left(\frac{\ln \left(\mathrm{t}^{\prime} / \overline{\mathrm{t}}\right)}{\mathrm{M}}\right)^{2}\right] \mathrm{dt}^{\prime}} .
$$

Notice that even with this simplified model where the stress amplitude is constant, Eq. (2.78) does not show a simple linear dependence on time.

Weibull Strength Distribution at a Fixed Stress Amplitude

We assume here that the strength level at a fixed lifetime, $N$, is given by a Weibull distribution function in the following form: 


$$
f(S)=\frac{\beta(S-\gamma)^{\beta-1}}{\eta^{\beta}},
$$

where the distribution is fully determined by three parameters: $\gamma, \beta$ and $\eta$. The probability distribution of the cycles to failure, $N$, then is given by

$$
f(N)=\frac{\beta b\left(\gamma^{\prime}-b \ln N\right)^{\beta-1}}{N \eta^{\beta}},
$$

where $\gamma^{\prime}=a-\gamma$. If the occurrence of stress cycles is at a fixed frequency, $r$, then the pdf is given by

$$
f(t)=\frac{\beta b\left(\gamma^{\prime}-b \ln (r t)\right)^{\beta-1}}{r \eta^{\beta}},
$$

and the failure rate finally is given by

$$
\lambda(t)=\frac{\left.\gamma^{\prime}-b \ln (\mathrm{rt})\right)^{\beta-1}}{\left.t \int_{t}^{\infty} t^{-1}\left(\gamma^{\prime}-b\right) \ln \left(r t^{\prime}\right)\right)^{\beta-1} d t^{\prime}} .
$$

\section{Random Stress and Known Strength}

On the basis of Miner's rule, the damage sustained in a single application of a given stress level, $S_{i}$, is $1 / N_{i}$. If the $N S^{b}=k$ relationship is used to approximate the $S-N$ curve, then this damage can be expressed as

$$
x_{i}=\frac{1}{N_{i}}=K S_{i}^{b}=g(S)
$$

If the magnitude of the applied stress, $S$, is random according to a distribution $f(s)$, the distribution of the damage, $x$, is

$$
f_{x}(x)=\left|\frac{d}{d x} g^{-1}(x)\right| f_{s}\left(g^{-1}(x)\right) \text {. }
$$

Then the distribution for the total damage, $D$, at time $t$ (where $D=\sum_{i} x_{i}$ ), assuming Poisson occurrences of stress, is

$$
f(D ; t)=\sum_{n=0}^{\infty} P_{n}(t) f_{n}(D)
$$

where $f_{n}(D)$ is the distribution for the total damage $D$ given $n$ stress applications, which is the $n$-fold convolution of $f(x)$. Therefore, the expected value of the damage at time $t$ is

$$
\bar{D}=\sum_{n=0}^{\infty} P_{n}(t) \int_{0}^{\infty} f_{n}(D) D d D=r \bar{x} t
$$


where $r$ is the rate of Poisson occurrences. Therefore, on the average, the damage accumulates linearly in time, as it should, because Miner's rule is a linear damage accumulation rule. Note that this is true only if it is assumed that the stress occurrences follow a homogeneous Poisson process. If a nonhomogenous Poisson process is assumed, the damage does not accumulate linearly in time on the average.

For Miner's rule, failure occurs when $D \geq 1$. Therefore, the distribution for the time to failure is

$$
F(t)=P(D \geq 1 ; t)=1-F_{D}(1 ; t)
$$

where

$$
F_{D}(D ; t)=\int_{0}^{D} f(\xi ; t) \xi
$$

and the failure rate is given by

$$
\lambda(t)=\frac{f(t)}{1-F(t)}=\frac{\frac{d}{d t} F_{D}(1 ; t)}{F_{D}(1 ; t)} .
$$

As a special case, assume that the amount of damage is fixed at $\bar{x}$ for every application of the stress, and let $[D / \bar{x}]$ be the largest integer less than $D / \bar{x}$. Then,

$$
F(D ; t)=\sum_{k=0}^{[D / \bar{x}]} e^{-r t} \frac{(r t)^{k}}{k !}
$$

To put this in terms of the derivation of the linear failure rate model described in Sec. 2.4.3, first note that the above analysis is consistent with that derivation, except for the assumption that the failure rate is proportional to the damage. In a physically based model, the failure rate is not assumed. Rather, what is assumed in the above physically based analysis is a model for the accumulation of damage, a model for the occurrences of stress, and a failure criterion. The failure rate follows directly from those assumptions, and it is not necessarily linearly increasing in time even though, on the average, the accumulation of damage is. Even in the case where the damage itself accumulates (deterministically) linearly with time, the failure rate is a complicated function of time.

\section{Random Stress and Random Strength}

For the general case in which both the stress and the strength are random, the stress-strength interference formula for the reliability distribution of a component subject to a cumulative damage process is expressed by Eq. (2.71), which is repeated here for convenience:

$$
R(t)=\sum_{i=0}^{\infty} \frac{e^{-\alpha t}(\alpha t)^{i}}{i !} \int_{0}^{\infty}\left(\prod_{j=0}^{i} F\left(y_{j}\right)\right) g\left(y_{0}\right) d y_{0}
$$

where the load applications are Poisson, $F(x)$ is the cumulative distribution of the load magnitude and $y_{j}$ is the strength at the $j$ th load application given by

$$
y_{i}=\psi(j) y_{0},
$$


where $\psi(\mathfrak{j})$ is a decreasing function of the load history.

In terms of Miner's rule for the accumulation of fatigue damage, the damage incurred at the ith stress, or load, application can be considered as

$$
y_{i}-y_{i+1}=\frac{1}{N\left(S_{i}\right)},
$$

where $y_{i}$ is the strength at the ith load application and $N(S)$ is the relationship between the stress level and the cycles-to-failure at that level as described by the S-N curve. Then, according to Miner's rule, failure occurs at the ith load application if

$$
\frac{1}{N\left(S_{i}\right)} \geq 1-\sum_{j=1}^{i-1} \frac{1}{N\left(S_{j}\right)}
$$

To cast this in terms of the SSI formula given above, the "strength" can be considered as the amount of damage the component can sustain from a given load application without failing. If the damage incurred is greater than that amount, the component fails. Therefore, if the load is considered to be the amount of damage incurred from a given stress application, if the stress at each load application, $S$, is random with a distribution $\mathrm{f}_{S}(\mathrm{~s})$, and if the damage increment is related to the stress by the typical formulation

$$
\frac{1}{N}=K S^{b}
$$

then the load distribution, $f(x)$, is

$$
f(x)=f\left(\frac{1}{N(S)}\right)=\frac{1}{b K^{1 / b}}\left(\frac{1}{N}\right)^{1 / b-1} f_{s}\left[\frac{1}{K^{1 / b}}\left(\frac{1}{N}\right)^{1 / b}\right]
$$

The damage function, $y(i)$, is then

$$
\psi(i)=\left(1-\sum_{j=1}^{i-1} y_{j}\right) \text {, }
$$

where $y_{j}=1 / N\left(S_{j}\right)$, and the reliability distribution is

$$
R(t)=\sum_{i=0}^{\infty} P_{i}(t) \int_{0}^{\infty}\left(\prod_{j=0}^{i} F\left(y_{j}\right) d y\right) g\left(y_{0}\right) d y_{0} .
$$

This obviously does not lead to a linearly increasing failure rate in general, even though the accumulation of damage is linear on the average.

\section{Probabilistic Fracture Mechanics}

To apply the framework discussed above to fatigue damage, the strength as a function of time must be characterized. In general, fatigue damage can be assumed to be directly related to the length of the fatigue crack. Therefore, given a crack length after a specified number of cycles, the strength must 
be related to the crack length through an appropriate fracture criterion. If the stress amplitudes are not time varying, this criterion can simply be a critical crack length determined from fracture mechanics principles, where failure occurs when the length of the fatigue crack exceeds the critical crack length. However, because the critical crack length depends on the magnitude of the applied load, a fracture criterion that relates the stress amplitude and the crack length to failure must be applied if the stresses are varying.

Thus, to estimate the fatigue reliability of a structure or component, four factors must be explicitly considered: the load history, the initial crack size distribution, the crack growth history as a function of the load history, and a failure criterion. Because there is much scatter in empirical crack propagation data, the crack growth rate must be modeled probabilistically.

There are three basic classes of probabilistic models for crack propagation. The most commonly used type involves randomization of an appropriate crack growth law, e.g., the Paris-Erdogan law. Other types of models include evolutionary probabilistic models (Markov or diffusion models) and cumulative jump models.

In crack-growth law-randomization models, appropriate quantities characterizing random effects are introduced into the empirical crack growth law. One approach to modeling random fatigue with empirical crack growth laws is to treat the parameters of the crack growth equation (the constants $C$ and $m$ in the Paris-Erdogan equation, for instance) as random variables, which can characterize the variability in stress range, material properties and environmental factors. Another approach is to introduce an appropriate stochastic process, $X(t)$, into the empirical law, i.e.,

$$
\frac{\mathrm{dA}}{\mathrm{dt}}=\mathrm{F}[\mathrm{A}, \Delta \mathrm{S}, \mathrm{C}, \ldots] \mathrm{X}(\mathrm{t})
$$

where $X(t)$ is a nonnegative random process representing the combined effects of the unknown random factors causing scatter in fatigue crack growth rates as a function of time. More information can be found in Lin et al. (1984), Ortiz and Kiremidjian (1988), and Sobczyk (1984).

The other types of probabilistic crack growth models will not be described here. They can be found in Sobczyk and Spencer (1992) (a review), Bogdanoff and Kozin (1985) (Markov models), Sobczyk and Trebicky (1989), and Ditlevsen and Sobczyk (1986) (cumulative jump models).

To estimate the fatigue reliability using a probabilistic crack growth model, a distribution of initial crack sizes must be specified, a stress history must be assumed, and a failure criterion must be formulated. The failure probability at time $t$ usually is computed via Monte-Carlo simulation. Examples of such calculations applied to nuclear reactor vessels and piping are described in Becher and Pedersen (1974) (vessel) and Harris and Lim (1982) (piping). Figure 2.1 shows the steps required to perform such a probabilistic failure analysis using the principles of fracture mechanics.

It can be concluded that in general, fatigue is not a linear damage accumulation process. Whether a linear approximation, such as Miner's rule, is reasonable in any given situation depends on the particulars of that application and the desired accuracy of the result.

\subsubsection{Irradiation Embrittlement}

\subsubsection{Introduction}

Irradiation embrittlement is the main degradation issue for PWR pressure vessels. The effect of neutrons from the nuclear reaction process bombarding the inside wall of the reactor vessel is a serious issue for maintaining the integrity of the pressure vessel. As a result of irradiation exposure, the 
ferritic steel strength increases with an attendant decrease in ductility. In terms of CVN energy properties, the ductile-to-brittle transition temperature (DBTT) increases to higher temperatures. Below the transition temperature, the material behaves in a brittle manner and its fracture requires only little energy, whereas above the transition temperature the material behaves in a ductile manner. Furthermore, the USE decreases in magnitude as the irradiation fluence increases. Decreases in the Charpy curve USE are associated with a decreased resistance to failure from ductile tearing. The effect of irradiation on the DBTT and USE is shown in Fig. 2.14.

\subsubsection{Correlations for the Ductile-to-Brittle Transition Temperature Shift}

In Revision 1 of NRC Regulatory Guide 1.99, the increase in the transition temperature (in ${ }^{\circ} \mathrm{F}$ ) as a result of neutron damage is given as

$$
\Delta \mathrm{RT}_{\mathrm{NDT}}=[40+1000(\mathrm{Cu}=0.08)+5000(\mathrm{P}-0.008)]\left(\frac{\mathrm{f}}{10^{19}}\right)^{1 / 2},
$$

where $\mathrm{Cu}$ and $\mathrm{P}$ are the weight percentages of copper and phosphorous, respectively, and $\mathrm{f}$ is the fluence in $\mathrm{n} / \mathrm{cm}^{2}$. This is thought to be overly conservative at high fluences.

Because of the increased understanding of the embrittlement process over the last several years, there have been a number of efforts to develop improved correlations to better predict the transition temperature shifts that were measured in the surveillance data base. A synthesis of correlations developed by Odette and Lombrozo (1984) and Guthrie (1983) form the basis for Revision 2 of NRC Regulatory Guide 1.99. The problem of predicting reduction in the CVN USE has not received much attention and is addressed in Revision 2 only in a perfunctory manner.

The Odette correlations for $\triangle \mathrm{RT}$ NDT are as follows.

$$
\begin{aligned}
\Delta \mathrm{RT}_{\mathrm{NDT}} & =360 \mathrm{Cu}\left[1+1.38\left(\operatorname{erf}\left[\frac{0.3 \mathrm{NiCu}}{\mathrm{Cu}}\right]+1\right)\right] \\
& \times\left[1-\exp \left(\frac{-\mathrm{f}}{0.11}\right)\right]^{1.36}\left[\mathrm{f}^{0.18}\right]
\end{aligned}
$$

for welds, and

$$
\Delta \mathrm{RT}_{\mathrm{NDT}}=389 \mathrm{Cu}\left[1+0.33\left(\operatorname{erf}\left[\frac{0.77 \mathrm{NiCu}}{\mathrm{Cu}}\right]+1\right)\right]\left[\mathrm{f}^{0.28}\right]
$$

for base metal.

The Guthrie correlations for $\triangle \mathrm{RT}_{\mathrm{NDT}}$ are as follows.

$$
\Delta R T_{N D T}=(624 \mathrm{Cu}-331 \sqrt{\mathrm{CuNi}}+251 \mathrm{Ni})\left[\mathrm{f}^{0.282-0.0409 \operatorname{lnf}}\right]
$$

for welds, and 


$$
\Delta \mathrm{RT}_{\mathrm{NDT}}=\left[-38+556 \mathrm{Cu}+480 \mathrm{Cu} \tanh \left(0.353 \frac{\mathrm{Ni}}{\mathrm{Cu}}\right)\right]\left[\mathrm{f}^{0.226-0.0449 \ln \mathrm{f}}\right]
$$

for base metal. The units of $\triangle R T$ NDT are in degrees Fahrenheit. Copper and nickel are given in per cent by weight and fluence, $f$, is in units of $10^{19} \mathrm{n} / \mathrm{cm}^{2}$.

\subsubsection{Pressure Vessel Failure Probability}

To estimate the probability of vessel failure under a postulated PTS transient, it is necessary to relate the shifts in the DBTT and USE to fracture mechanics material properties. At lower temperatures in the brittle regime, the linear-elastic crack initiation toughness, $\mathrm{K}_{\mathrm{Ic}}$, and crack arrest toughness, $\mathrm{K}_{\mathrm{Ia}}$, can be used. However, at higher temperatures elastic-plastic parameters for characterizing the toughness of steel are needed. Stable crack growth resistance curves $(\sigma-R$ curves) often are used for characterizing the upper-shelf toughness, $J_{c}$, and a considerable effort has been made to evaluate the effect of neutron irradiation on them.

Lower bound curves for the relationship of the fracture toughness to temperature (for both $\mathrm{K}_{\mathrm{Ic}}$ and $\mathrm{K}_{\mathrm{Ia}}$ ) are given in Appendix A of Sec. XI of the ASME Boiler and Pressure Vessel Code. The effects of irradiation on the crack initiation and arrest fracture toughness can be estimated by applying the shift in the RTNDT to shift the ASME lower bound curves for $\mathrm{K}_{\mathrm{Ic}}$ and $\mathrm{K}_{\mathrm{Ia}}$, by moving the curves by the same shift amount, but leaving the shapes unaltered. This relationship can be written as

$$
\begin{aligned}
& \mathrm{K}_{\mathrm{Ic}}=33.2+2.806 \exp \left(0.02\left[\mathrm{~T}-\mathrm{RT}_{\mathrm{NDT}_{0}}-\Delta \mathrm{RT}_{\mathrm{NDT}}+100\right]\right), \\
& \mathrm{K}_{\mathrm{Ia}}=26.78+1.233 \exp \left(0.0145\left[\mathrm{~T}-\mathrm{RT}_{\mathrm{NDT}_{0}}-\Delta \mathrm{RT}_{\mathrm{NDT}}+160\right]\right) .
\end{aligned}
$$

Given relationships like those described above, the probability of vessel failure can be estimated as outlined in Sec. XI, Appendix A, of the ASME Boiler and Pressure Vessel Code. Given a postulated transient, the pressure in the vessel as a function of time and the temperature inside the vessel as a function of time and location in the vessel can be calculated; from that the crack initiation and arrest toughnesses can be determined as a function of time and crack tip position. If an initial crack size and location are assumed, the crack propagation is assumed to initiate when the stress intensity factor at the crack tip, $\mathrm{K}_{\mathrm{I}}$, exceeds the initiation fracture toughness, $\mathrm{K}_{\mathrm{Ic}}$, and will propagate until $\mathrm{K}_{\mathrm{I}}<\mathrm{K}_{\mathrm{Ia}}$, or until the crack completely penetrates the vessel. At the end of the transient, if the crack has propagated to beyond a critical length, the vessel is assumed to have failed.

If some of the parameters of the deterministic methodology described above are treated as random variables, the probability of vessel failure because of a particular transient can be estimated. Jouris and Witt (1983) describe a probabilistic methodology in which copper content, phosphorous content, $\mathrm{K}_{\mathrm{IC}}$ and $\mathrm{K}_{\mathrm{Ia}}$, are treated as random variables. The probability of vessel failure because of a large loss-of-coolant accident (LOCA) or large steam line break are calculated for a variety of initial flaw sizes. It was found that the copper content is the most important parameter. The Vessel Integrity Simulation Analysis (VISA-II) code, described in Simonen and Engel (1986) uses Monte Carlo simulation to evaluate the failure probability of a PWR vessel subjected to a PTS transient specified by the user. A version of the code was used to evaluate the sensitivities of calculated through-wall flow probability to material, flow, and calculational assumptions. Alternative flow assumptions changed the probabilities by one to two orders of magnitude (Simonen and Johnson, 1986a and b). The OCA-P code, developed at ORNL and used in the NRC's Integrated Pressurized Thermal Shock project also uses 
Monte Carlo simulation to estimate the failure probability of a PWR reactor pressure vessel subject to pressurized thermal shock (Cheverton and Ball, 1984).

More recent studies include a phenomenon known as warm prestressing. An essential characteristic of warm prestressing relative to PTS is that $\mathrm{K}_{\mathrm{I}}$ at first increases and then monotonically decreases with time. When $\mathrm{K}_{\mathrm{I}}$ is decreasing, a crack will not propagate, even if $\mathrm{K}_{\mathrm{I}}$ 》 $\mathrm{K}_{\mathrm{Ic}}$.

The approach based on linear elastic fracture parameters described above is unsuitable to model ductile tearing and mixed mode crack propagation. In the upper-shelf temperature regime, crack initiation would be a result of ductile tearing. Crack initiation occurs when the J-integral exceeds the ductile tearing fracture initiation toughness, JIc. Eason and Nelson (1991) have related changes in the Charpy USE to changes in the J-R curves and JIC.

\subsubsection{Probabilistic Model for Pressure Vessel Reliability}

The probability of failure, $\mathrm{p}$, of a pressure vessel containing defects is given by

$$
\mathrm{p}=\int_{\mathrm{K}_{\mathrm{Im} \text { in }}}^{\mathrm{K}_{\mathrm{Im} \text { ax }}} \mathrm{P}_{1}\left(\mathrm{~K}_{\mathrm{I}}\right)\left[\int_{0}^{\mathrm{K}_{\mathrm{I}}} \mathrm{P}_{2}\left(\mathrm{~K}_{\mathrm{Ic}}\right) \mathrm{dK}_{\mathrm{Ic}}\right] \mathrm{dK}_{\mathrm{I}}
$$

where $\mathrm{P}_{1}\left(\mathrm{~K}_{\mathrm{I}}\right)$ is the pdf for the stress intensity factor and $\mathrm{P}_{2}\left(\mathrm{~K}_{\mathrm{Ic}}\right)$ is the pdf for fracture toughness. In the case of (LEFM), the pdf, $\mathrm{P}_{1}\left(\mathrm{~K}_{\mathrm{I}}\right)$, is given by

$$
\mathrm{P}_{\mathrm{I}}\left(\mathrm{K}_{\mathrm{I}}\right)=\mathrm{GP}_{3}(\sigma) \sqrt{\pi \mathrm{a}}
$$

where $P_{3}(\sigma)$ is the pdf for applied stress, $G$ is a factor dependent on the defect shape and local geometry, and $a$ is the defect depth. On the other hand, when plastic yield is considered ahead of the crack tip (PYFP), $\mathrm{K}_{\mathrm{I}}$ is given by

$$
\mathrm{K}_{\mathrm{I}}=\sigma_{\mathrm{u}} \mathrm{G}\left[\frac{8 \mathrm{a}}{\pi} \ln \sec \left(\frac{\sigma \pi}{2 \sigma_{\mathrm{u}}}\right)\right]^{1 / 2} \text {, }
$$

where $\sigma_{\mathfrak{u}}$ is the ultimate tensile strength. The term in the above equation is a correction to the crack length term in Eq. (2.66) to account for plasticity ahead of the crack tip. by

The pdf for the applied stress in the general membrane area of a nuclear pressure vessel is given

$$
\mathrm{P}_{3}(\sigma)=\frac{\mathrm{A}}{\sigma} \exp \left(-\frac{\mathrm{B}}{\sigma}\right)
$$

where A and B are constants. Substituting Eq. (2.111) into Eqs. (2.109) or Eq. (2.110) will give the pdf for $\mathrm{K}_{\mathrm{I}}$. On the other hand, from Eqs. (2.101) and (2.102), one can obtain a pdf for $\mathrm{K}_{\mathrm{Ic}}$ and $\mathrm{K}_{\mathrm{Ia}}$. A pdf for $\triangle \mathrm{RT}$ NDT can be assumed to be Gaussian, i.e.,

$$
\mathrm{f}\left(\Delta \mathrm{RT}_{\mathrm{NDT}}\right)=\frac{1}{\sqrt{2 \pi \alpha_{2}}} \exp \left[\frac{\Delta \mathrm{RT}_{\mathrm{NDT}}(\phi \mathrm{t})}{2 \alpha_{2}^{2}}\right] \text {, }
$$


where $\Delta \mathrm{RTNDT}(\phi t)$ is an increasing function of neutron fluence (or time), as discussed earlier. It is clear from Eqs. (2.112) and (2.101) that $\mathrm{K}_{\mathrm{Ic}}$ will be a decreasing function of time and that when Eq. (2.108) is used, the probability of failure, $\mathrm{p}$, will be an increasing function of time. When the methods of reliability assessment given in Sec. 2.4 are used, it is clear that the failure rate is a function of time. Obviously, the time dependence is quite nonlinear. However, it remains to be seen whether such time dependence can be approximated as quasi-linear.

\subsubsection{Stress-Corrosion Cracking}

As discussed in Sec. 2.5.1.3, SCC is a form of environmentally assisted cracking that can affect various reactor components, e.g., steam generator tubes.

The combination of a corrosive environment and a tensile stress creates conditions whereby existing cracks can grow. Furthermore, small changes in environmental and stress conditions can have a marked effect on the crack growth process. This leads one to conclude that the variability observed in SCC between nuclear plants is to be expected. A discussion of the current understanding of SCC can be found in Parkins (1992).

\subsubsection{General Deterministic Models}

Mathematically, the models describing SCC and fatigue crack growth are similar. The data resulting from SCC tests have been presented in both S-N and the fracture mechanics (crack growth rate vs stress intensity) formats (Parkins, 1979) as described in Sec. 2.5.3. A discussion of the fracture mechanics formulation follows and is based on the presentation by Wei (1979).

\section{Crack Propagation Models}

From a fracture mechanics point of view, SCC and fatigue crack growth are behaviorally similar, i.e., a stress field created at the crack tip causes crack propagation. This can be seen in Fig. 2.18, where the crack growth rate vs stress intensity for fatigue and SCC are shown. Note that for fatigue the time variable of interest is measured in number of cycles, whereas for SCC, it is measured in time units. Note also that the stress intensity $\mathrm{K}$ for $\mathrm{SCC}$ is based on a single tensile stress, whereas the stress intensity, $\Delta \mathrm{K}$, is based on an alternating stress for fatigue crack propagation.

\section{Paris-Erdogan Formula}

\section{Model Objectives:}

The Paris-Erdogan formulation is based on the empirical model used to analyze fatigue crack propagation. It is empirical in nature and relates the crack growth rate to the stress intensity factor.

\section{Model Formulation:}

The mathematical expression for SCC crack growth in the Paris-Erdogan formulation can be written as (Congleton and Craig, 1982, and Wei, 1979)

$$
\frac{\mathrm{da}}{\mathrm{dt}}=\mathrm{CK} \mathrm{I}_{\mathrm{I}}^{\mathrm{n}} \text {, }
$$

where $a$ is the crack length, $K_{I}$ is the stress intensity at the crack tip, $t$ is time, $C$ is a constant, and $n$ is an exponent characterizing the material, environment, temperature, etc. The stress intensity $\mathrm{K}_{\mathrm{I}}$ is given by (Ford, 1982) 

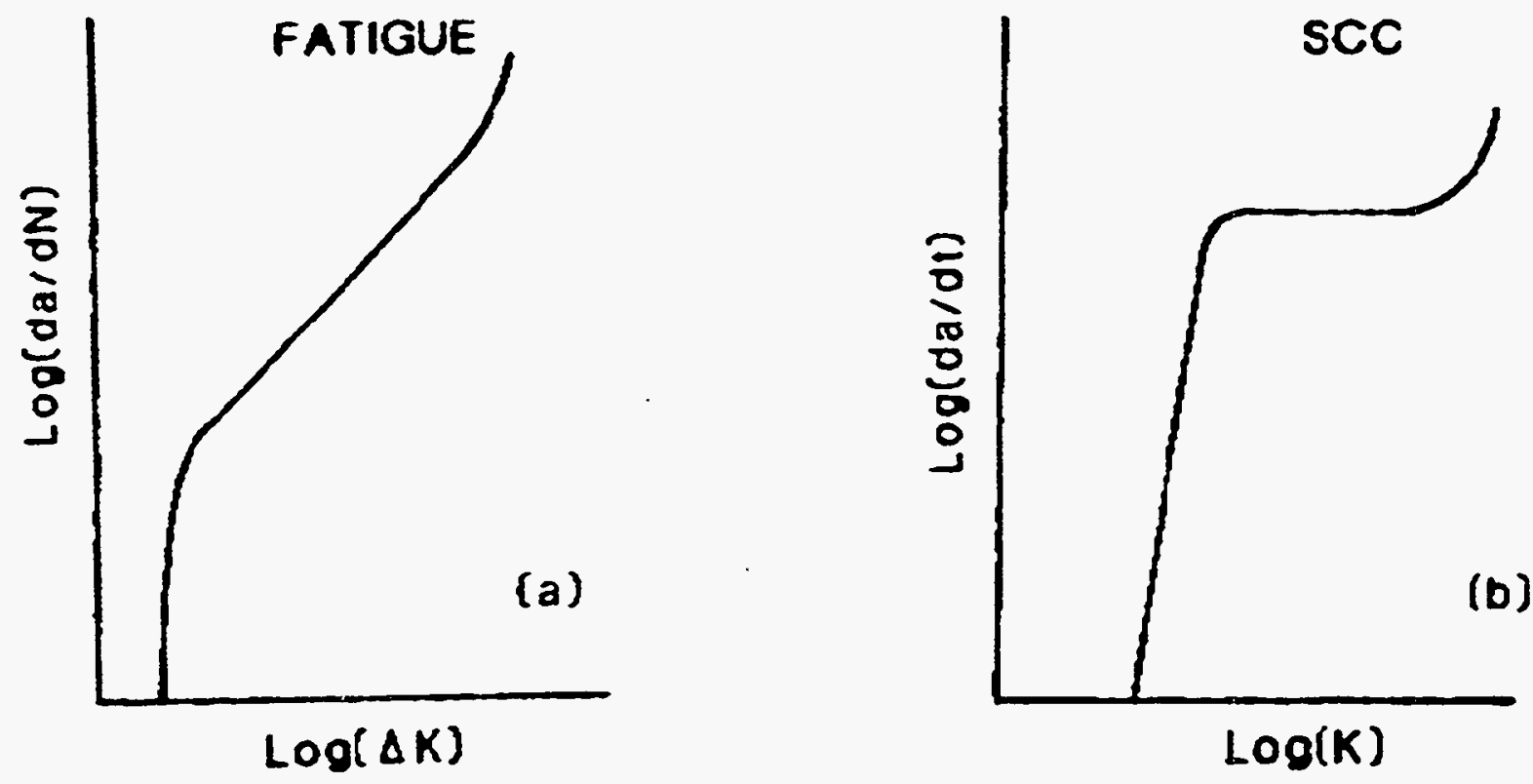

Figure 2.18 Fatigue and stress-corrosion cracking (Congleton and Craig, 1982) 


$$
\mathrm{K}_{\mathrm{I}}=A \sigma \sqrt{\frac{\pi \mathrm{a}}{\mathrm{B}}}
$$

where $\mathrm{A}$ and $\mathrm{B}$ are constants depending on geometry and $\sigma$ is the far-field tensile stress.

As the crack grows, the value of $\mathrm{K}_{\mathrm{I}}$ changes. Failure occurs when $\mathrm{K}_{\mathrm{I}}=\mathrm{K}_{\mathrm{IC}}$, where $\mathrm{K}_{\mathrm{IC}}$ is the fracture toughness of the material.

\section{Assumptions:}

The Paris-Erdogan formulation assumes that SCC can be modeled in a manner similar to fatigue crack propagation. It also assumes that the crack growth rate depends only on the stress intensity and that LEFM assumptions are met.

\section{Discussion:}

Figure 2.19 shows a schematic of the failure time for SCC vs the stress intensity. Figure 2.19 also shows that no SCC is presumed to occur below a threshold value of $\mathrm{K}_{\mathrm{I}}$, denoted as $\mathrm{K}_{\mathrm{ISCC}}$. The relative value of $\mathrm{K}_{\mathrm{ISCC}}$ to $\mathrm{K}_{\mathrm{C}}$ is a property of the material. The incubation time is defined arbitrarily to be that period during which the rate of crack growth is less than $10^{-6} \mathrm{in} . / \mathrm{min}$. The length of time for which this is the case depends on $\mathrm{K}_{\mathrm{I}}$, the material, and the environment and can vary substantially. The time to failure is given by the sum of the incubation time and the crack growth time obtained through the curve shown in Fig. 2.19.

\subsection{Conclusions}

In this section, various reliability models, especially those that could be applied to aging, have been reviewed and discussed. Special attention has been paid to the linear aging reliability model proposed in Vesely (1987). A primary purpose of this task was to evaluate the suitability of the linear aging reliability model for estimating the reliability and/or unavailability of nuclear power plant systems, structures and components which are susceptible to aging related degradation. Also reviewed was the Taylor expansion approach proposed in Vesely (1990) for incorporating the increase in component unavailability because of aging into an existing plant risk model. following.

With respect to the Taylor expansion approach to aging risk evaluation, we concluded the

(1) Aging may increase the importance of terms that had been judged as negligible, or had not been accounted for, in the basic plant risk model. Most important in this regard is the possible increase in the failure probabilities of major passive components such as the reactor pressure vessel and the reactor coolant piping.

(2) The assumption that changes in component unavailabilities are independent may not be valid if the stressors that cause aging introduce potential common-cause failures that had not been considered in the baseline risk model.

(3) Even though the model is presented in terms of changes in component unavailabilities, the only contribution to the unavailabilities currently considered is that resulting from changes in the failure rates. In fact, expressing the model in terms of changes in failure rates would be more appropriate because it would avoid problems arising from the same parameter appearing in two or more contributors to system unavailability. 

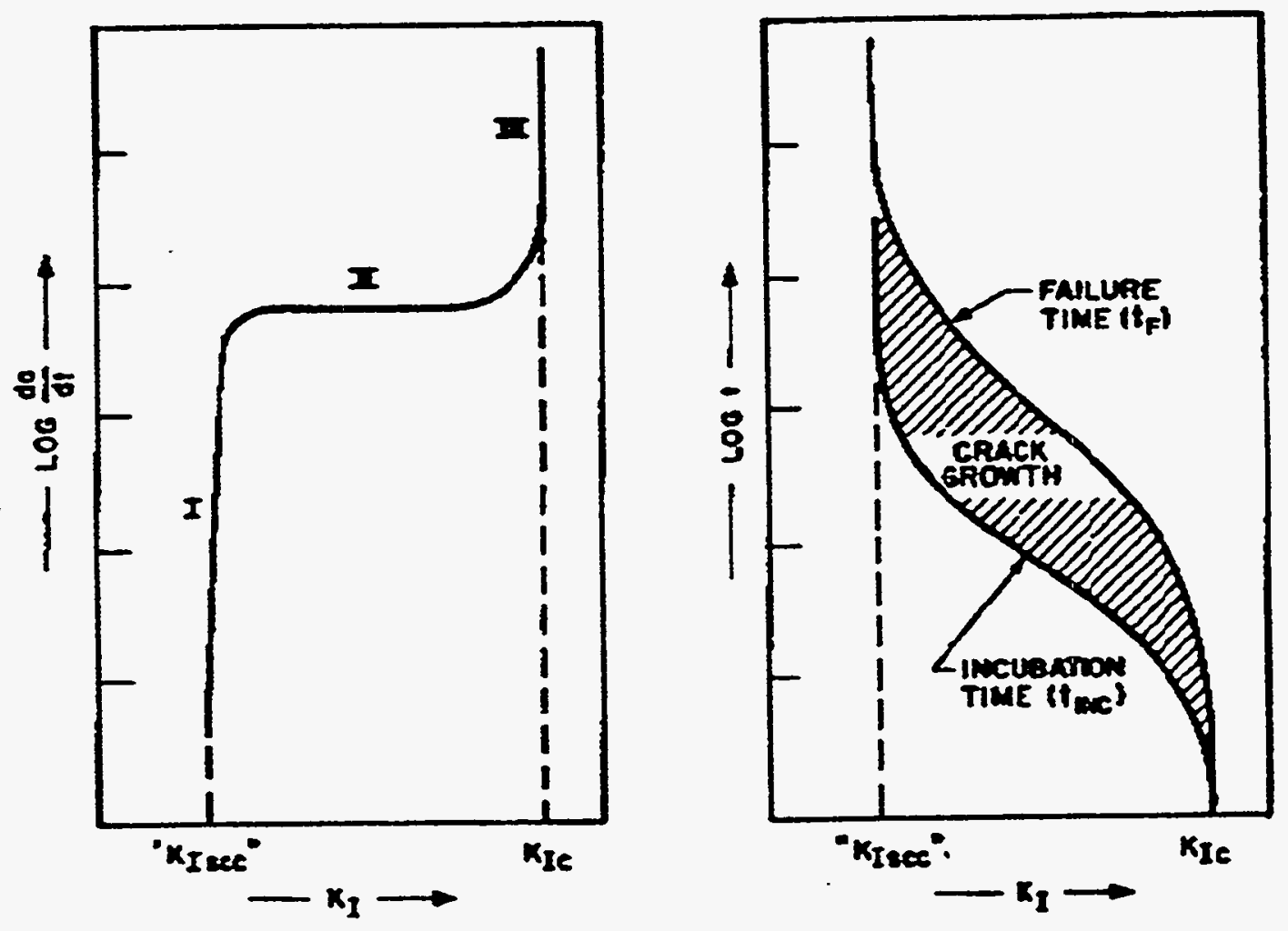

Figure 2.19 Schematic representation of crack growth and time to failure (Wei, 1979) 
(4) However, contributions to component unavailabilities other than changes in the failure rates are not considered even with the above modification. Other contributions that could be important include factors such as the increase in the maintenance contribution to the unavailability because of the increase in the frequency of repair and maintenance outages of aged components.

(5) Considering the effect of aging on risk through component unavailabilities is too restrictive because there are situations where terms other than component unavailabilities may contribute to risk.

(6) The restriction to component unavailabilities and failure rates masks the physics behind various contributors to risk, thus inhibiting risk management.

With respect to the linear aging reliability model, it was determined that a linear failure rate model, in general, is not applicable to many aging-related degradation mechanisms. Even if the accumulation of damage is approximately linear, this does not imply that the failure rate is linearly increasing. Further detailed modeling of the specific damage accumulation mechanisms and the conditions that lead to failure must be carried out to determine whether and under what conditions the linearly increasing failure rate model is a reasonable approximation.

A major drawback of directly assuming a form for the failure rate (linearly increasing or not), is that many important physical phenomena and quantities may be obscured. This masking of the physics of the problem affects aging management in a significant way because the possibilities of interfering with the physical mechanisms and the effect of such interference are obscured. For example, working with failure rates only does not allow the effect of an improved crack detection probability on the reliability of a pipe to be evaluated. The only way to do this is to work with models that deal explicitly with crack growth as discussed in Sec. 2.5.

Three major degradation mechanisms are discussed in this report: fatigue, irradiation embrittlement, and stress-corrosion cracking. With the available information, it could not be determined whether the linear aging reliability model is a reasonable approximation to the reliability of components experiencing these degradation mechanisms, although it is obvious that in general it does not apply rigorously. For fatigue in particular, it has been shown that although the damage accumulation because of fatigue may be, and commonly is in design applications, modeled as approximately linear, this does not lead to a linearly increasing failure rate

The main benefit of the linear aging reliability model is its simplicity and ease of application. If it is determined that this model is a reasonable approximation to the reliability of components experiencing aging related degradation, then it is indeed a superior model. However, its suitability for modeling most of the important degradation mechanisms has yet to be determined because of a lack of actual failure data and detailed calculations based on physical fundamentals. Of course, even if this model proves to be reasonable under certain conditions, it would still suffer from its inability to provide estimates of the effect of specific aging management strategies on the probability of failure of components subject to aging mechanisms of a known physical nature. 



\section{REVIEW OF AGING DATA ANALYSES}

The statistical methods used in aging data analysis and the data used in nuclear plant aging analyses are reviewed in this section. Data used in plant aging analyses are discussed in Secs. 3.1-3.3, and statistical methods for describing and evaluating the effects of aging on component lifetimes are discussed in Sec. 3.4.

Under the Nuclear Plant Aging Research (NPAR) Program (USNRC, 1991), aging assessments have been conducted for more than 40 categories of SSCs that were considered to be risk-significant to NPP safety. As shown in Fig. 3.1, the pre-Phase-I study of the NPAR selected the SSCs to be examined in the program, and both deterministic and probabilistic approaches were used in the selection process. Phase I focused on identifying the potential failure modes and the degradation sites, mechanisms, and stressors of the SSCs. The functional parameters that can be used as indicators of the status and aging progression of the component also were identified. Another activity of Phase I was to evaluate the inspection, surveillance, monitoring, and maintenance (ISMM) methods that are currently used at NPPs. Phase II of the NPAR program included comprehensive tests and laboratory research that might lead to the improvement of ISMM practices and better methods for assessing the residual service-life of the structure or equipment.

Data used in the NPAR program range from generic and plant-specific operating experience, plant documents, test and research results, and expert judgments to industrial practice. These data sources cover a wide spectrum and are subject to various kinds of limitations. For example, although data obtained from laboratory tests are often considered as "objective" sources, they may present only partial glimpses and may be conducted with some constraints. At the other extreme, al though expert judgments are often used to draw conclusions on broader issues, they can be very subjective and they are subject to biases and large uncertainty. Because the decisions made on aging issues are sensitive to the data input, it is important to have a clear understanding of how these data are derived and how reliable they are. The objectives of this section are to review the data used in different phases of the NPAR program, discuss the availability and the quality of the data sources, and comment on the conclusions drawn from these data.

\subsection{Data Used in the Prioritization and Selection of Systems, Structures, and Components in the NPAR Program}

In 1986, the Executive Director for Operations (EDO) of the Nuclear Regulatory Commission established the Technical Integration Review Group for Aging and Life Extension (TIRGALEX) (Levy et al., 1988) to structure a plan to integrate the NRC's aging and life-extension activities. One of the

major results of the TIRGALEX plan has been the identification of the safety-related structures and components that should receive high priority in the subsequent phase of the NPAR program.

The TIRGALEX expert panel used a set of risk criteria called the "Risk Significance of Component Aging" (RSCA) (Vesely, 1988) and the "Risk Significance of Component Aging and Aging Management Practices (RSCAAMP)" to set the priorities of the aging evaluation of the structures and components. The same data set used in this process, which has since been called the TIRGALEX data base, was used as the database in later probabilistic risk studies (Vesely et al., 1990, and Vesely, 1992) under the NPAR program.

The TIRGALEX data base is the result of an expert panel workshop held in May 1987. It provides the input necessary to conduct the RSCA, which is an evaluation of a component's contribution to plant risk because of aging, and RSCAAMP, which is an evaluation of the effectiveness of current industry-management practices for maintaining an acceptable risk level of plant in the presence of 


\section{NPAR Program Strategy}

PRE-PHASE $1 \cdot$ paIORITIzaTION

OF CS
PHASE I - MTERIM AGINO ASSESSMENT OF CS

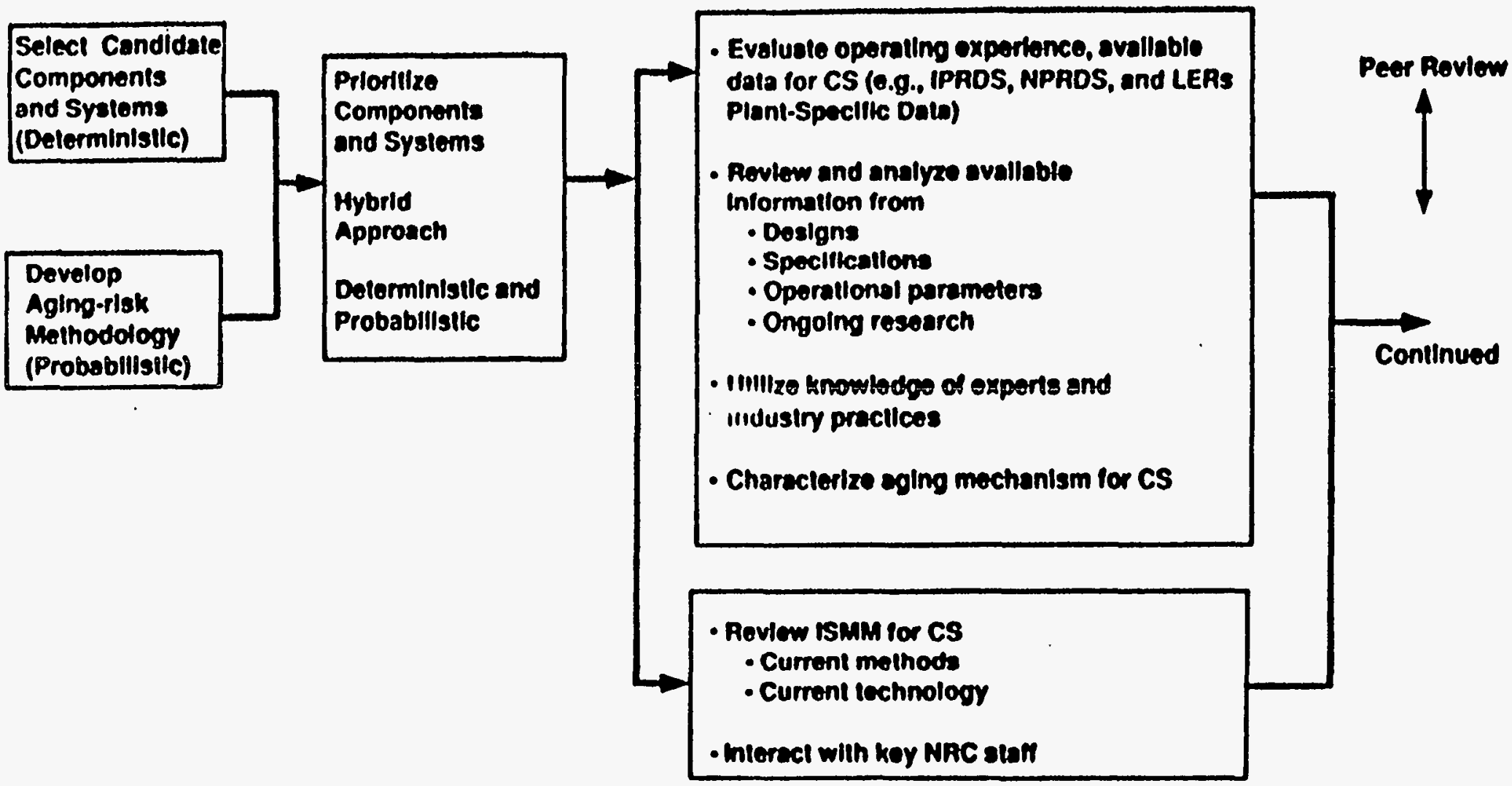

CS = Components Systems 


\section{NPAR Program Siraiegy}

PHASE $n$ - COMPAEHENSNE AGHO ASSESSMENT OF CS - OPTIONAL APPROACHES
UTIRATION Of RESEARCH AESULTS

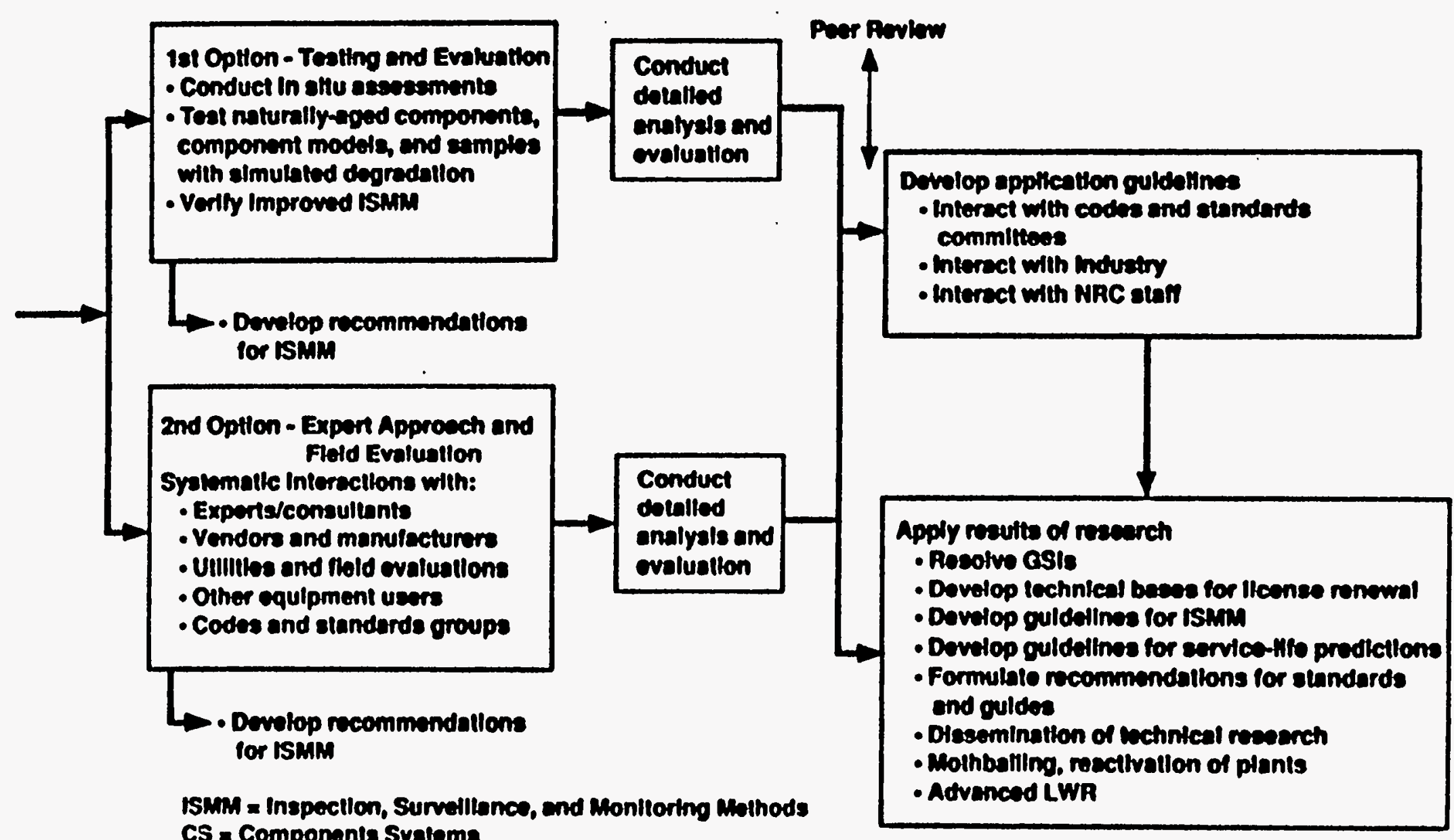

CS = Components Syatems 


\section{NPAR Program Strategy}

\section{PHASE I ACTIVITIES}

1. Prefliminary Identificallon of susceptibility. of matorials to ege-related degradation.

2. Idenilification of stressors and related environmental factors thol could cause age-related degradation.

3. Identincation of degradation allea.

4. Idontincation of fallure modos and causeas.

5. Dovilopment of functional parameters to Indicale present stafus and aging progresslon.

6. Evaluation of current inspection, survelitance, and monitoring methods.

7. Evaluation of current malntenance prectices.

a. Rocommendatlons for Phase II studtes.

\section{PHASE II ACTIVITES}

1. In sltu assesamont of naturally-ged CS.

2. Tests of naturally-ged components or tests of components with slmulated aging.

3. Laboratory of in-plant verfication of methods of inspection, monlioring, and survelliance.

4. Verimcation of methods of residual corvicotilio ovaluation.

5. Development of recommendations for inspoctlon or monitoring mothods and pertods.

6. Pocommendations for effective maintenance practlces.

7. Fecommendattons for acceptable methods to determine restdual life of equipment 
aging components. In the basic RSCA model, the change in a component's contribution to risk because of aging is expressed as

$$
\Delta \mathrm{R}=\mathrm{N} * \alpha \frac{\mathrm{L}^{2}}{2},
$$

where $\Delta \mathrm{R}$ is the change in plant risk as a result of the aging of a structure or a component, $\mathrm{N}$ is the normal risk importance associated with the component, $\alpha$ is the increase in failure rate because of component aging, and $\mathrm{L}$ is the interval (between overhauls) during which the component is aging. The normal risk importance, also called Birnbaum measure or reliability importance, measures the increase of plant risk resulting from the increase of the unavailability of a specific component. It is a measure of the critical relationship to the system in which it resides. The RSCA approach assigns a higher priority to the structure or component that would introduce higher change in plant risk because of component aging.

One major assumption used in deriving Eq. (3.1) is that it uses the linear aging model (Vesely, $1987)$, in which the time-dependent failure rate is expressed as

$$
\lambda(t)=\lambda_{0}+\alpha t
$$

where $\lambda(t)$ is the time-dependent component failure rate and $\lambda_{0}$ is the component failure rate associated with random failures. All of the discussion given earlier in this report regarding the use of linear aging model should apply to the RSCA model.

To include the effectiveness of aging management, the change in a component's contribution to risk because of aging has been extended in the RSCAAMP model as follows:

$$
\Delta \mathrm{R}=\mathrm{N} * \alpha * \frac{1}{2}\left(\frac{\mathrm{L}_{\text {ind }}}{\mathrm{P}_{\mathrm{D}} \times \mathrm{P}_{\mathrm{R} / \mathrm{D}}}\right)^{2}
$$

where $L_{\text {ind }}$ is the surveillance or test interval that is representative of current industry practices, $P_{D}$ is the probability of successfully detecting degradation resulting from aging of the component during the surveillance or test interval, and $P_{R / D}$ is the probability of successfully mitigating the aging degradation process given its successful detection. Although the values of $P_{D}$ and $P_{R} / D$ may depend on the degree of component degradation caused by aging, the model assumes that they are constant over time.

TIRGALEX data for $N, \alpha,{ }^{*}$ ind,$P_{d}$, and $P_{R / D}$ can be categorized into three parts according to their sources and derivation procedures: the $N$ and $A$ values for the PRA-based components; the $N$ and $A$ values for the non-PRA-based components; and the values for $L_{i n d}, P_{d}$, and $P_{R} / D$. The PRA-based component data in TIRGALEX were derived from pre-calculated values from the existing PRAs, whose data are largely based on operating experience and on engineering judgment. The TIRGALEX expert panel reviewed these data and made changes on the precalculated numbers to reach the final set of data for these PRA-based components.

\footnotetext{
In the TIRGALEX data base (Levy et al. 1988), the notation $A$ is used instead of $\alpha$.
} 
The second part of the TIRGALEX data includes $\mathrm{N}$ and $\mathrm{A}$ values for those structures and components that generally are not included in PRAs. Because of the lack of information from operating experience, the data for these non-PRA-based components are based solely on expert judgment. The third part of the TIRGALEX data consists of the estimates of the effectiveness of aging control (i.e., $L_{\text {ind, }} P_{D}$, and $P_{R / D}$ ) and also are based largely on expert judgment.

\subsubsection{TIRGALEX Precalculated Data for the PRA-Based Components}

Four PRAs from three U.S. nuclear-power-plant vendors were selected as the basis for the generic risk importance (N) in TIRGALEX data for PRA-based components. The median values of the four PRA risk-importance values were presented to the panel, and the panel made changes from the precalculated values as necessary.

The aging failure-rate increases (A) in this part were estimated based on information in the INEL data base that was generated specifically for the NPAR program. The increase in failure rate because of aging is calculated by applying the method of moments and is represented by a function of three variables: the aging fraction ( $f$ ) (i.e., the fraction of failures determined to be caused by agingrelated stressors), the mean time-to-aging failure $\left(T_{A}\right)$, and the component failure rate $(\lambda)$ :

$$
A=\frac{4 \lambda f}{3 T_{A}} .
$$

The aging fractions were taken from Meale and Sutterwhite (1988), which is a large-scale analysis of aging data performed by INEL using the Nuclear Plant Reliability Data System (NPRDS) data base. More than 2000 NPRDS records from reactors of three vendors (B\&W, Westinghouse, and GE) on 15 safety-related systems were reviewed in the INEL study, and the causes of these failures were evaluated to determine the fraction of failures that were caused by various age-related stressors. The failure rates $(\lambda)$ were assigned best-estimate values from various data sources. The mean time-toaging-failures ( $\mathrm{T}_{\mathrm{A}}$ 's) also were obtained from Meale and Sutterwhite (1988).

The calculated increases in failure rate because of aging and the failure-rate doubling times were presented to the TIRGALEX expert panel. Based on these precalculated values and on other information available, the panel then came up with estimates of the increases in the failure rate. The decisions made on these final values are often subjective. For example, a precalculated A for batteries was $3.4^{*} 10^{-8}$, which gives an average of 29.2 yr for failure rate doubling time. The panel agreed that batteries were susceptible to aging failure mechanisms and a 29.2-yr doubling time was too long. The panel settled with a doubling time of $3 \mathrm{yr}$, which yields a $3.4^{*} 10^{-7}$ for A. Table 3-1 shows the set of TIRGALEX data for A and other parameters.

\subsubsection{TIRGALEX Data for Non-PRA-Based Components}

There are structures and components that are not included in PRA studies. Examples are RPV supports and Category I concrete structures and some major equipment, e.g., the RPV and cables, connectors, and penetrations. The values of $\mathrm{N}$ and $\mathrm{A}$ for these non-PRA-based components are based solely on the judgment of the TIRGALEX panel. For example, in the case of the structures or components whose major roles are the mitigation of accident sequences, the importance was assigned to be 1.0, because the panel considered that these structures or components should be set to the highest level of risk significance. The bases for these subjective assignments were recorded carefully so that they could be retrieved easily if necessary. 
Table 3-1 Factor values and final ranking of structures/components (Levy et al., 1988)

\begin{tabular}{|c|c|c|c|c|c|c|c|c|}
\hline & Component & $\begin{array}{c}n \\
\text { (c o/yr) }\end{array}$ & $\left(h r^{-1} r^{-}\right.$ & $\begin{array}{l}\text { Lind } \\
(50 .) \\
\end{array}$ & $P_{D}$ & $P_{R D}$ & $\begin{array}{c}\text { Risk } \\
\text { Increase } \Delta R \\
\text { (C } D / y r)^{(b)} \\
\end{array}$ & $\begin{array}{l}\text { Final } \\
\text { Bonk }\end{array}$ \\
\hline $\begin{array}{r}5 . \\
10 . \\
2 . \\
10 . \\
6 . \\
19 . \\
17 . \\
12 . \\
17 . \\
18 . \\
19 . \\
5 . \\
16 . \\
24 .\end{array}$ & 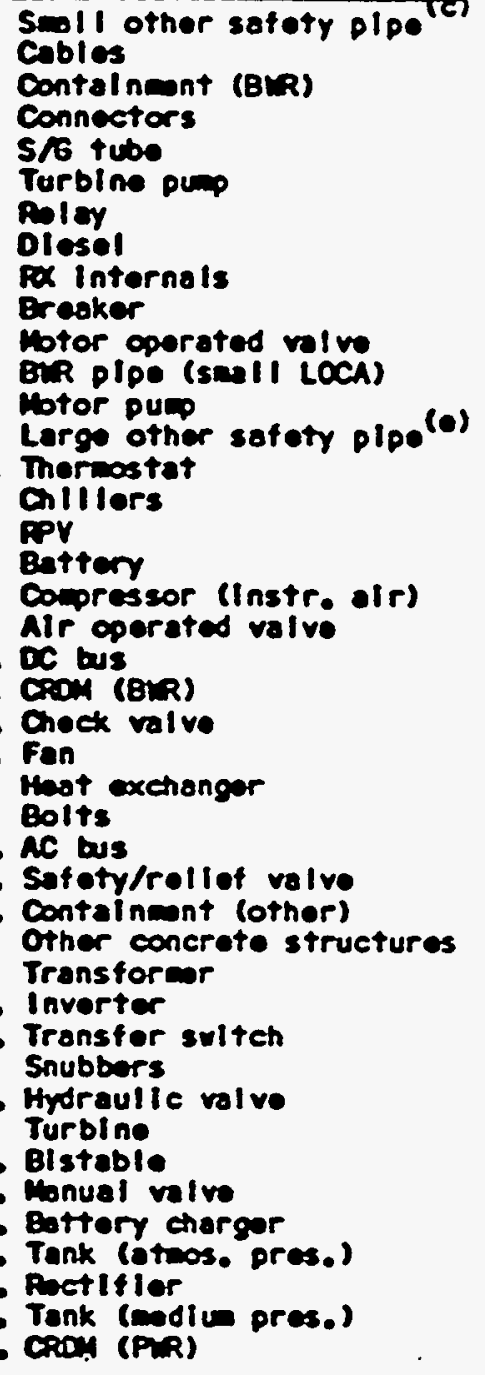 & 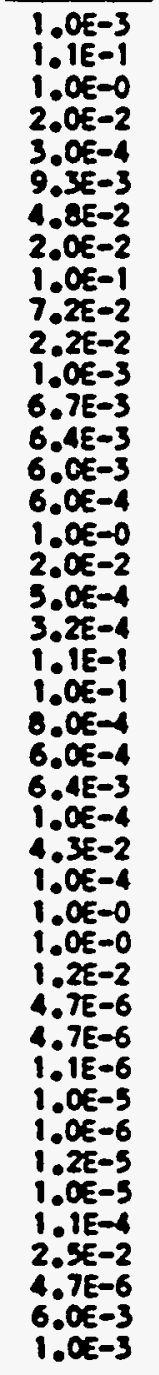 & $\begin{array}{l}3.0 E-7 \\
2.7 E-9 \\
1.0 E-7 \\
2.7 E-8 \\
5.0 E-6 \\
2.7 E-6 \\
2.5 E-7 \\
3.6 E-6 \\
2.0 E-9 \\
1.6 E-8 \\
3.6 E-6 \\
3.0 E-8 \\
2.2 E-7 \\
3.0 E-9 \\
1.5 E-7 \\
1.5 E-6 \\
2.0 E-12 \\
3.4 E-7 \\
5.0 E-7 \\
4.0 E-7 \\
1.1 E-9 \\
3.0 E-9 \\
3.0 E-9 \\
2.1 E-7 \\
1.4 E-8 \\
5.1 E-7 \\
1.1 E-9 \\
6.7 E-7 \\
1.0 E-13 \\
1.0 E-13 \\
1.7 E-9 \\
1.9 E-6 \\
2.3 E-7 \\
5.1 E-6 \\
1.3 E-7 \\
1.0 E-7 \\
1.0 E-7 \\
2.2 E-9 \\
3.5 E-8 \\
2.0 E-12 \\
8.7 E-8 \\
1.0 E-12 \\
3.0 E-11\end{array}$ & $\begin{array}{r}60.0 \\
60.0 \\
18.0 \\
60.0 \\
36.0 \\
12.0 \\
6.0 \\
3.0 \\
18.0 \\
18.0 \\
3.0 \\
36.0 \\
12.0 \\
18.0 \\
18.0 \\
18.0 \\
120.0 \\
6.0 \\
6.0 \\
18.0 \\
18.0 \\
18.0 \\
18.0 \\
18.0 \\
3.0 \\
18.0 \\
18.0 \\
18.0 \\
60.0 \\
60.0 \\
18.0 \\
12.0 \\
18.0 \\
18.0 \\
18.0 \\
60.0 \\
18.0 \\
60.0 \\
12.0 \\
12.0 \\
12.0 \\
12.0 \\
18.0\end{array}$ & $\begin{array}{l}0.10 \\
0.10 \\
0.90 \\
0.20 \\
0.50 \\
0.50 \\
0.20 \\
0.30 \\
0.10 \\
0.50 \\
0.70 \\
0.20 \\
0.50 \\
0.10 \\
0.70 \\
0.70 \\
0.90 \\
0.90 \\
0.20 \\
0.50 \\
0.50 \\
0.90 \\
0.10 \\
0.70 \\
0.10 \\
0.50 \\
0.50 \\
0.90 \\
0.10 \\
0.10 \\
0.70 \\
0.50 \\
0.20 \\
0.90 \\
0.50 \\
0.90 \\
0.70 \\
0.50 \\
0.90 \\
0.10 \\
0.50 \\
0.10 \\
0.50\end{array}$ & $\begin{array}{l}0.90 \\
0.90 \\
0.80 \\
0.90 \\
0.90 \\
0.90 \\
0.90 \\
0.90 \\
0.90 \\
0.90 \\
0.90 \\
0.90 \\
0.90 \\
0.90 \\
0.90 \\
0.90 \\
0.10 \\
0.90 \\
0.90 \\
0.90 \\
0.90 \\
0.90 \\
0.90 \\
0.90 \\
0.90 \\
0.90 \\
0.90 \\
0.90 \\
0.50 \\
0.90 \\
0.90 \\
0.90 \\
0.90 \\
0.90 \\
0.90 \\
0.90 \\
0.90 \\
0.90 \\
0.90 \\
0.70 \\
0.90 \\
0.70 \\
0.90\end{array}$ & 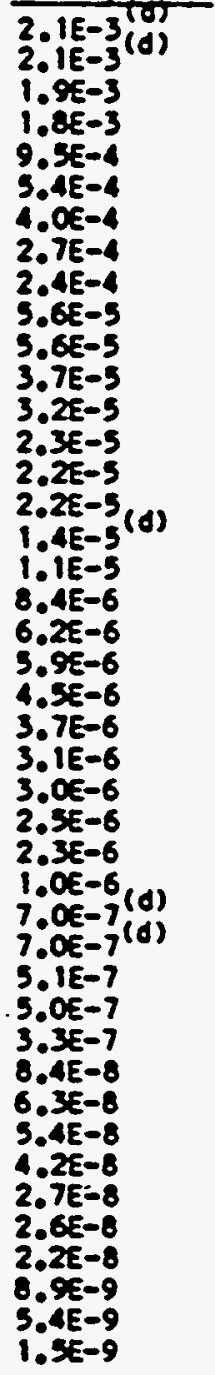 & $\begin{array}{l}3 \\
5 \\
5 \\
5 \\
4 \\
4 \\
1 \\
3 \\
3 \\
3 \\
3 \\
3 \\
3 \\
3 \\
3 \\
3 \\
3 \\
2 \\
2 \\
2 \\
2 \\
2 \\
2 \\
2 \\
2 \\
2 \\
2 \\
2 \\
1 \\
1 \\
1 \\
1 \\
1 \\
1 \\
1 \\
1 \\
1 \\
1 \\
1 \\
1\end{array}$ \\
\hline 28. & 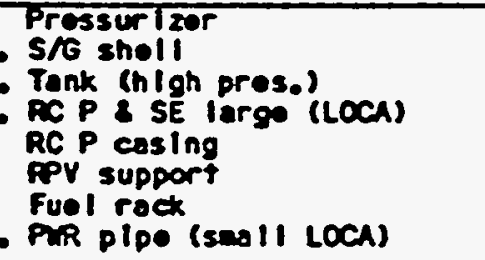 & 1.0 & $\begin{array}{l}1.0 E-12 \\
1.0 E-12 \\
1.0 E-12 \\
1.0 E-12 \\
1.0 E-12 \\
1.0 E-12 \\
5.0 E-12 \\
1.0 E-12\end{array}$ & $\begin{array}{l}18.0 \\
= \\
= \\
=\end{array}$ & $\begin{array}{l}0.90 \\
= \\
= \\
= \\
=\end{array}$ & $\begin{array}{l}0.90 \\
= \\
= \\
=\end{array}$ & $\begin{array}{l}1.2 E-12 \\
= \\
= \\
= \\
=\end{array}$ & $\begin{array}{l}7 \\
1 \\
1 \\
1 \\
1 \\
1 \\
1 \\
1\end{array}$ \\
\hline
\end{tabular}

(a) Coution: only the relative component position (rank) is Ieportant; do not base any subsequent studies on the numprical values presented here.

(b) To obtaln, the units for $\Delta R$, (C O/Yr), $A$ is uitiplied by $8760 \mathrm{hrs}^{-1}$ and $\mathrm{Lr}^{-1}$ ind asitiplied

(c) by 12 yr (both $P_{0}$ and $P_{R} Q$ are probabllililes).

(d) L for these components was truncated to a mxlmin of 40 peors (480 months).

(e) I0E\$4 in. plpe represented oy service water plping (se Tabie 2.2 ).

(1) Components bion this IIne wre olininated from further consideration by sercening oriterlon

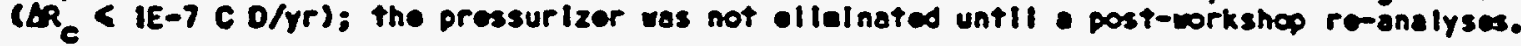




\subsubsection{TIRGALEX Data for Aging Management}

The TIRGALEX data for $L_{\text {ind }}$ (the surveillance or test interval that is representative of current industry practices), $\mathrm{PD}_{\mathrm{D}}$ (the probability of successfully detecting aging degradation in the component within the surveillance or test interval), and $P_{R / D}$ (the probability of successfully mitigating the aging degradation process, given its successful detection) also are based solely on expert judgment. For example, the panel felt that for many mechanical and electrical components, the current surveillance/ operability tests that were required by technical specifications were of limited value in identifying the aging-related failure modes and that those being identified usually were found through tests conducted during refueling outages in conjunction with preventive maintenance activities. As a result, many components were assigned an 18-month $L_{\text {ind }}$ (a typical interval between refueling outages) in the TIRGALEX data base.

For $\mathrm{PD}_{\mathrm{D}}$, the current surveillance/test methods for many components were judged by the panelists to be ineffective in detecting aging; therefore, the values for $P_{D}$ are low (in the 0.1-0.3 range) in many cases. However, the values for $P_{R} / D$ are generally high because the panel thought that the probability of successfully mitigating a risk significant aging mechanism when it was detected was rather high. Again, these assignments are subjective, but they have been recorded carefully for easy retrieval.

\subsubsection{Discussion of the TIRGALEX Data}

TIRGALEX data have been used to prioritize components for further aging research (Levy et al., 1988 ) and to evaluate the effectiveness of maintenance practices in controlling aging phenomena (Vesely et al., 1990). As can be seen from the discussion in the previous sections, TIRGALEX data are based on limited PRA studies, operating experience recorded in the NPRDS data base, and to a great extent, expert judgment. In the following paragraphs, we comment on the assignment of values to TIRGALEX factors, the use of TIRGALEX data in making decisions on aging-related issues, and, finally, the RSCA and its alternative formalisms. In developing these comments, we have benefited from a discussion with Dr. R. J. Budnitz, chairman of the TIRGALEX expert panel, and a letter addressed to Dr. Ira Levy.

(1) Assigning values for the risk importance, N. For PRA-based components, the risk importance values that the expert panel assigned were based on a set of precalculated numbers, which were the median values of the risk importance from four PRA studies (Table 3-2). One immediate observation is that these values are plant-specific and, hence, vary significantly from plant to plant. Furthermore, the expert panel often viewed the importance of the PRA-based components differently from those that might have been inferred from the precalculated values. The differences between the pre-calculated PRA results and the panel's expert judgment can be seen from the differences in the values used for the importance values in the final TIRGALEX data base (Table 3-1) and the precalculated values (Table 3-2). One major reason for such differences, as pointed out in Budnitz's letter, is that the $N$ value of a TIRGALEX group was too broad for the assignment of a single $N$ value, and sometimes different $N$ values would apply to different failure modes. For the risk importance of non-PRA-based components, only expert judgment, which sometimes led to an arbitrarily assigned high value $(\mathrm{N}=1)$, was used.

(2) Assigning values for the change of component failure rate, A. The rates of change of component failure rates for PRA-based components are based on information provided in the NPRDS data base. Because the records are filed on a voluntary basis, the NPRDS historically has had problems related to a lack of completeness and consistency. For the non-PRA-based components, the increases in failure rates are based on expert judgment. 
Table 3-2 Four-plant component risk importance data by component type (Levy et al., 1988)

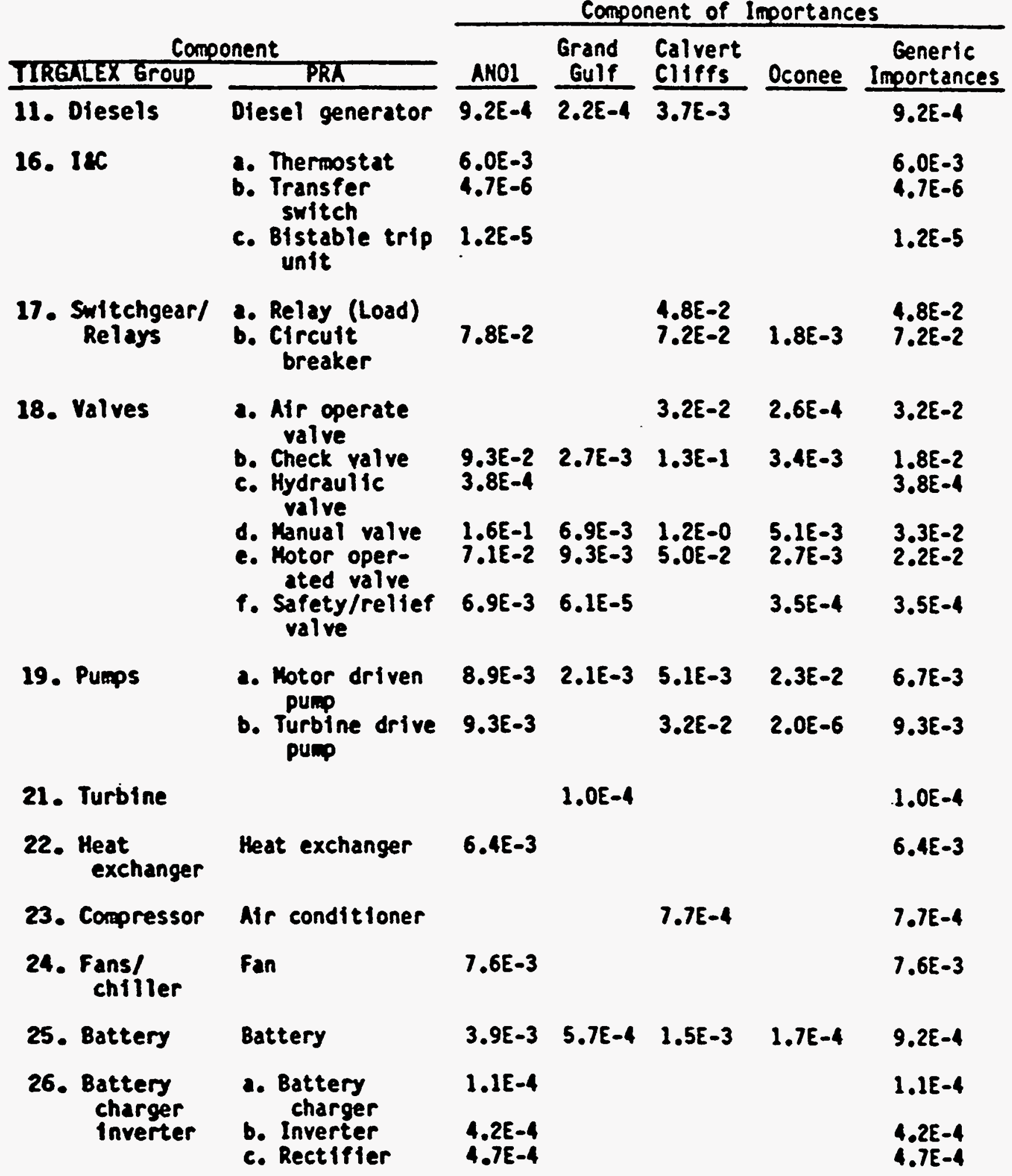


Table 3-2 Four-plant component risk importance data by component type (Levy et al., 1988) (Cont.)

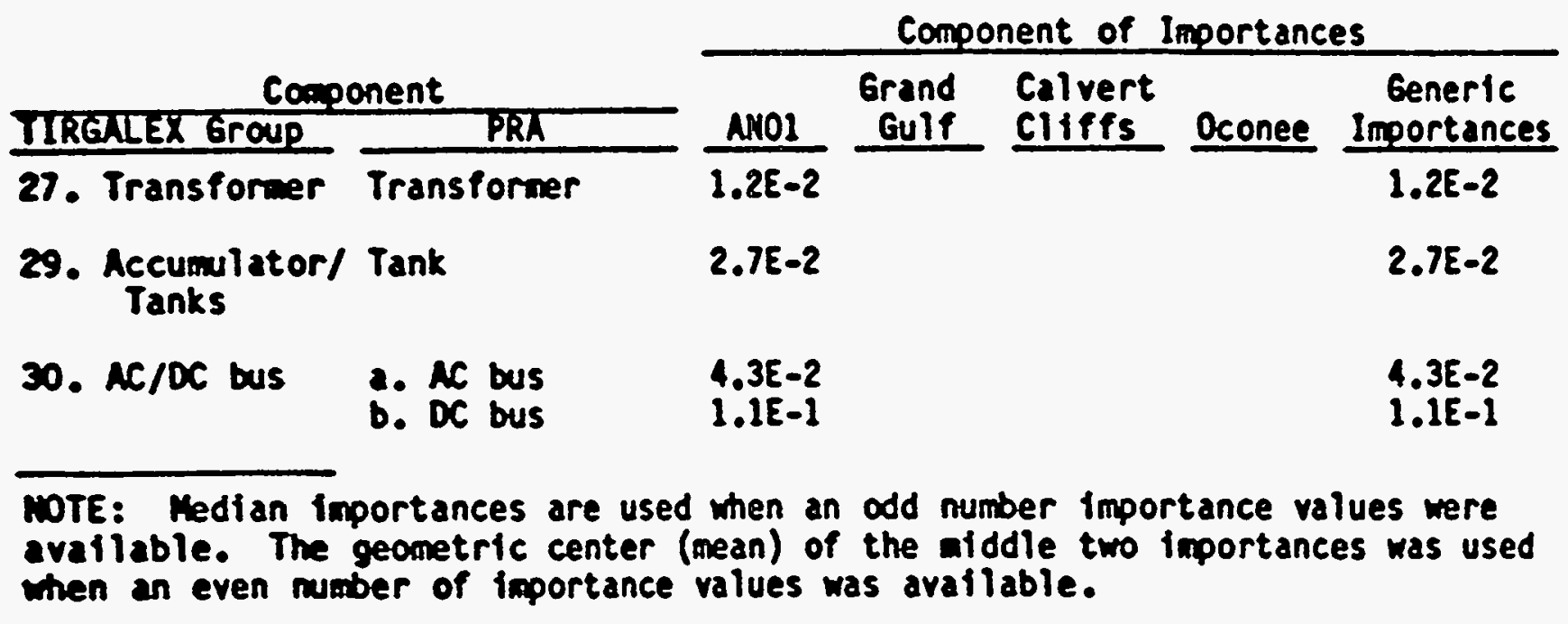


One difficulty of assigning an $\mathrm{A}$ is that a component might experience more than one aging mechanism and have more than one failure mode. Furthermore, the RSCA formalism assumes that component aging follows a linear aging model, which is a point of view not shared by all experts.

(3) Assigning values for $L_{i n d}, P_{D}$, and $P_{R / D}$. TIRGALEX data on maintenance practices for aging control are based solely on expert judgment. The main difficulty here is that these values are plant-specific and, in the cases of $P_{D}$ and $P_{R} / D$, mechanism-specific. Because the RSCA formalism takes the form $\left(1 / P_{D}{ }^{*} P_{R} / D\right)^{2}$, the panel was reluctant to assign small values to $P_{D}$ and $P_{R} / D$, even though they felt that the current industrial practices might be able to catch and "fix" some of the aging mechanisms while not being able to arrest some of the others at all.

(4) The use of expert judgment in determining TIRGALEX data. No formal procedures in the elicitation, debiasing, and aggregation of expert opinions (Chibber et al., 1992 and Cooke, 1991) were applied in the TIRGALEX data assessment. The TIRGALEX panel did not receive the same amounts of resource and assistance in the formalisms and instruments designed to evaluate expert opinion numerically as, for example, the panels of NUREG1150 received. Although the panel might have provided useful qualitative insights, the users of TIRGALEX data should be especially aware of the limitations of the numerical values assigned to those factors.

(5) The use of TIRGALEX data in setting priorities in NPP components for aging research. Limited by the RSCA formalism (see below), the TIRGALEX panel was more concerned about the overall ranking of the components in order to set priorities in aging research than the specific numbers assigned to the individual factors. The users of TIRGALEX data should realize this crucial fact and be careful when these factors are applied individually.

(6) The use of TIRGALEX data in making other decisions. The use of a highly subjective and uncertain database raises more concerns in evaluating the effectiveness of the maintenance practices and aging management practices. As NUREG/CR-5510 (Vesely et al., 1990) uses plant-specific importance values to evaluate the effectiveness of maintenance practices, the TIRGALEX importance data do not affect the conclusions in that report. Furthermore, only PRA-based components are included in the maintenance evaluation; therefore, the non-PRA-based data do not affect the conclusions reached in the NUREG/CR-5510 analyses. A typical recommendation made in the study is to reduce the maintenance and surveillance test interval from $x$ months to $y$ months. Because the recommendations are based on a set of highly subjective data, it will be hard to find justification for them. Furthermore, because non-PRA-based components are not included in the analysis, inspection, and maintenance practices conducted on the passive components (for example, piping and structures) are not evaluated in these studies. Another point is that although large uncertainty exists in the estimated parameters, the TIRGALEX data base only provides point estimates and the expert panel spent no effort trying to address the issue of uncertainty nor any effort to keep track of the disagreement among experts. Depending on the purpose of using the TIRGALEX data, the uncertainty distribution may be one of the important factors affecting decision-making.

(7) The RSCA and alternative formalisms. The RSCA model considers the effect of plant risk from aging by taking the components one at a time. In reality, components age simultaneously. This effect of the aging of multiple components has been addressed in NUREG/CR-5510 (Vesely, 1990) and NUREG/CR-5587, in which a Taylor series expansion 
has been used to replace the RSCA model. The other feature of NUREG/CR-5510 and NUREG/CR-5587 is that plant-specific data, instead of industrial averages, were used for risk importance and test intervals in the analyses. Aging rates for some components also were modified either to reflect more recent knowledge or to conduct sensitivity analyses. Neither RSCA nor the Taylor series expansion models provide reliable answers. The existing approaches lack a formalism designed for the elicitation and processing of expert opinions and using them in aging-related decision making.

\subsection{Operating Experience Used in Aging Assessments}

The NPAR aging assessment covers the study of more than 40 SSCs that are considered risksignificant. The objectives of these aging assessments are to identify the aging-related degradation sites and mechanisms, to review the ISMM practices, and to identify the performance parameters or condition indicators that are potentially useful in detecting degradation. A large portion of the components considered are active components (pumps, valves, etc.) for which operating experience can be found from industrial and plant-specific data bases. The rest are passive components (piping, structures, etc.) for which the aging-related degradation and aging mechanisms can be identified only through special inspection techniques, such as nondestructive examination. In this section, we discuss the use of industrial and plant-specific operating experiences in identifying aging mechanisms in active components. Data on material properties for fracture mechanics analyses are discussed in Sec. 3.3.

An important data source for the aging assessments is the operating experience reported in public and private data bases, vendor information, the open literature, utility sources, and expert opinion. In this section, we review some of these data sources that have been used in aging assessments.

\subsubsection{Generic Data Bases}

The most often cited generic data bases include the following.

(1) Nuclear Plant Experience (NPE) (S. M. Stoller Corp.). Compiled by the S. M. Stoller Corporation, NPEs are accumulations of operating experiences of U.S. light-water reactors. The summaries of NPEs include details of component failures, causes, and corrective actions of more than 50,000 selected events on both BWRs and PWRs.

(2) Licensee Event Reports (LERs). The LER system is basically a compilation of specific events reported to the NRC as part of regulatory requirements.

(3) Nuclear Plant Reliability Data System (NPRDS). This is an industry-wide voluntary effort, under the supervision of the Institute for Nuclear Power Operations (INPO), to provide the operating statistics of components in safety-related systems.

(4) Other component- or system-specific data bases. For example, the Emergency Diesel Generator Component Tracking System (EDGCTS), a data base assembled by the Transamerica Delaval, Inc. (TDI) diesel-engine owners' group, was used in the interim aging assessment of nuclear-station diesel generators (Hoopingarner et al., 1987).

\subsubsection{Limitations on the Use of Generic Data Bases}

There are several major limitations regarding the use of generic data bases in aging studies.

(1) The most serious problem encountered in using generic data bases is that none is comprehensive enough to provide all the information that is needed to make an aging assessment. For example, although NPE gives a fairly detailed discussion on the causes 
and corrective actions of component/system failures, the selection of the events to be analyzed is subjective. A similar problem exists with the NPRD system. Because the failure information in NPRDS is reported on a voluntary basis, completeness is always a question. On the other hand, although LER reporting is mandatory, only events of a limited number of failure types (typically those events involving the violation of a Technical Specification) are included in the data base. Furthermore, the LERs do not contain detailed information (such as root causes) and the corrective actions taken even this information is crucial to the aging data analyses.

(2) In general, the existing generic data bases do not provide information on details associated with aging and their mitigating measures. This creates uncertainty and difficulty in determining the aging mechanisms and the frequency of their occurrence.

(3) The aging phenomena of equipment are often plant-specific and depend much on the quality of the specific manufacturer, the installation and the operating condition of the equipment, and the maintenance routines performed at a specific site. Aging-related failure rates derived from generic data bases frequently do not represent plant-specific conditions.

(4) The reporting requirements and practices of the generic data bases have changed throughout the years. Furthermore, the circumstances (such as the attitude of plant managers toward a specific data system) surrounding the submittal of events may have also changed over time. Therefore, the increase in component failure rates over the years is possibly as much the result of circumstantial factors as it is of aging mechanisms.

\subsubsection{Plant-Specific Data}

Plant-specific maintenance data are used in conjunction with the generic data in the NPAR aging assessment. There are both merits and limitations with the use of plant-specific data. The corrective and preventive maintenance records kept at each nuclear plant often contain more detailed information regarding the component maintenance history, causes of failures, mitigation measures, and corrective actions; therefore, they provide information that is more applicable to the plant operating environment. As with the generic data bases, the problems lie in the completeness of the information and its susceptibility to changes of circumstantial surroundings. Another potential problem with the plant-specific data is that the quality of maintenance records varies not only from plant to plant but also from year to year. This increases the uncertainty in the quality of the results of a data analysis based on plant-specific data.

Another limitation of using plant-specific data in aging assessments is that the number of incidents is often too small to reach a meaningful statistical conclusion. This is especially true for new plants with a short operating history.

\subsubsection{DORIAN: A Software Package for Bayesian Aging Data-Analysis}

A Bayesian approach is used in the computer software DORIAN, which was developed by PLG to evaluate aging trends in equipment failure rate, maintenance rate, and maintenance duration (Bier et al., 1991; Bier et al., 1990a, 1990b, and 1990c; Bley et al., 1991). In this approach, the data analyst specifies subjectively a prior probability distribution indicating the degree of belief that the analyst has over a range of equipment failure rates and over the trends of the change of failure rates over time. Plant-specific data on actual component failures over time are collected and are used to update the prior probability distribution. 
Figure 3.2 shows the concept of the Bayesian aging approach. The prior probability distribution is assigned over the initial failure rates $\lambda(t=0)$ and a set of aging models. In Figure $3.2, j$ distinct linear aging models are postulated over $\mathrm{i}$ distinct initial failure rates. The posterior distribution of $\lambda$ at year 60 , using the plant-specific failure experience, is shown on the right. Figure 3.3 shows the kind of plant-specific data collected for a DORIAN exercise.

The results of applying DORIAN to plant-specific data from maintenance records of two systems of a BWR plant with $17 \mathrm{yr}$ of operating experience indicate a slight tendency toward increasing failure rates for some components and a slight tendency toward decreasing failure rates for some others. Plant-specific data definitely rule out the likelihood of a rapid change in failure rate because of aging at this specific plant.

\subsubsection{Expert Opinions}

All aging assessments under the NPAR program use the judgments of experts. Sometimes these experts are the operators and maintenance staff who have had direct and day-to-day experience with the systems or components of concern. Experts from the manufacturing sectors and/or from other industries with relevant experiences also are called on to address the aging problems.

The selection of the experts in the NPAR program seems to be subjective, and for most of the cases, the elicitation processes for expert opinions are conducted informally. The issues of bias and aggregation of expert opinions remain major problems when assigning numerical values (such as failure rates and the probability of detection).

\subsubsection{Discussion}

A general conclusion reached regarding the use of data from operating experience in assessing component aging is that although a large amount of information is available regarding the reliability and the aging phenomena of active components, the level of uncertainty in the results of the analyses of the aging data remains high. Points worth discussing in this regard include the following.

(1) No single data source, generic or plant-specific, provides the necessary detail to perform a detailed analysis of component aging analysis. Generic data bases have the limitations of instability and lack of completeness. However, by using a plant-specific data base only, as pointed out in the aging data analysis for the service water-system aging-degradation assessment (Jarrell, 1989), it is likely that one would not be able to identify most root causes or failure mechanisms with even a low degree of confidence.

(2) A combination of various sources may allow a qualitative evaluation of a representative sample of available data and the derivation of useful perspective on the failure modes, causes, aging mechanisms, and some preliminary corrective actions. However, it is doubtful that any quantitative conclusions can be reached, even with a limited degree of confidence. Because of the sparse and unreliable nature of the existing data sources of detailed failure information, most studies have simply left out any quantitative conclusions.

(3) In many cases, additional difficulties in the data analyses have been introduced because some vendors, utilities, and manufacturers have been reluctant to provide the necessary information. Some may consider certain information as proprietary, and some may have negative attitude toward research work generally and the aging research specifically.

(4) Data from operating experience are not sufficient for validating any of the proposed aging models (linear, exponential, or the Weibull aging model). 


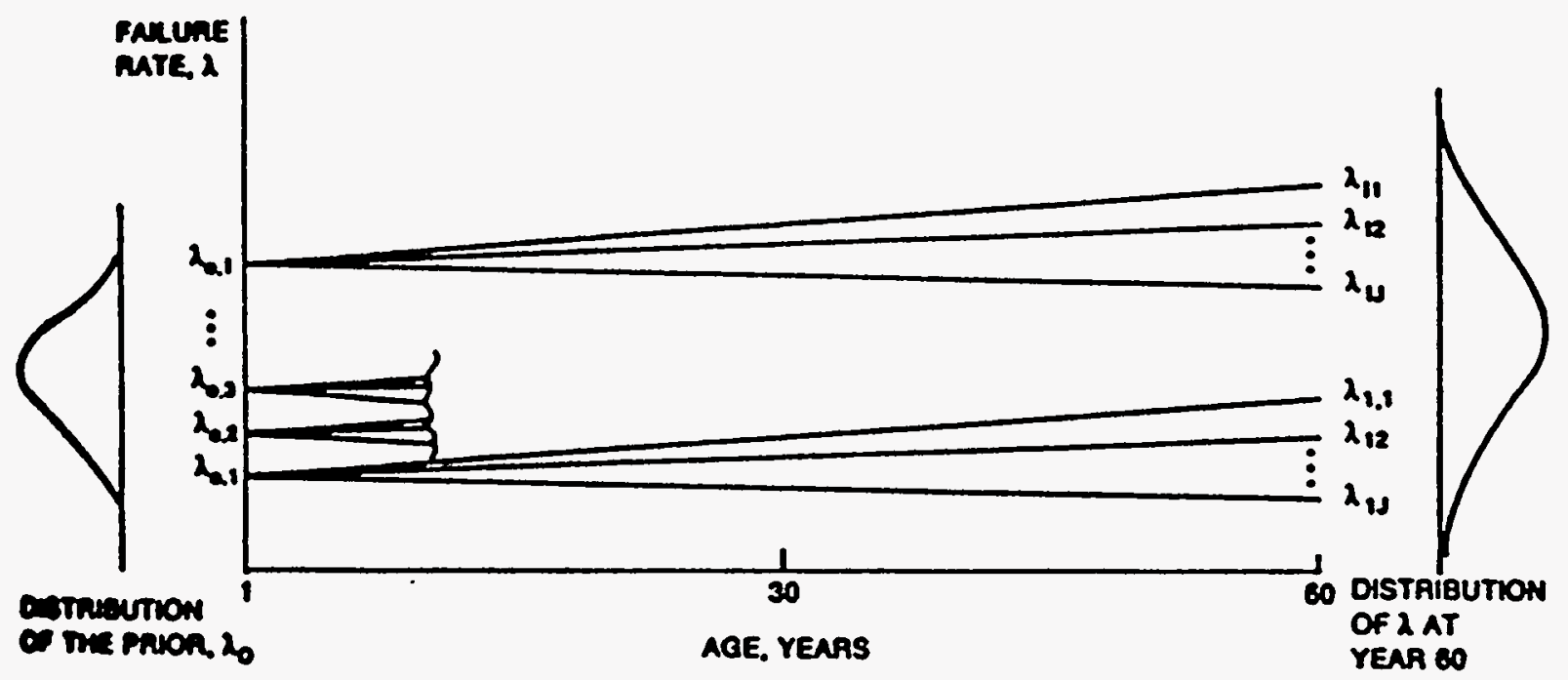

Figure 3.2 The prior and posterior distributions of a component failure rate expressed in a set of linear aging models (Bier et al., 1991) 


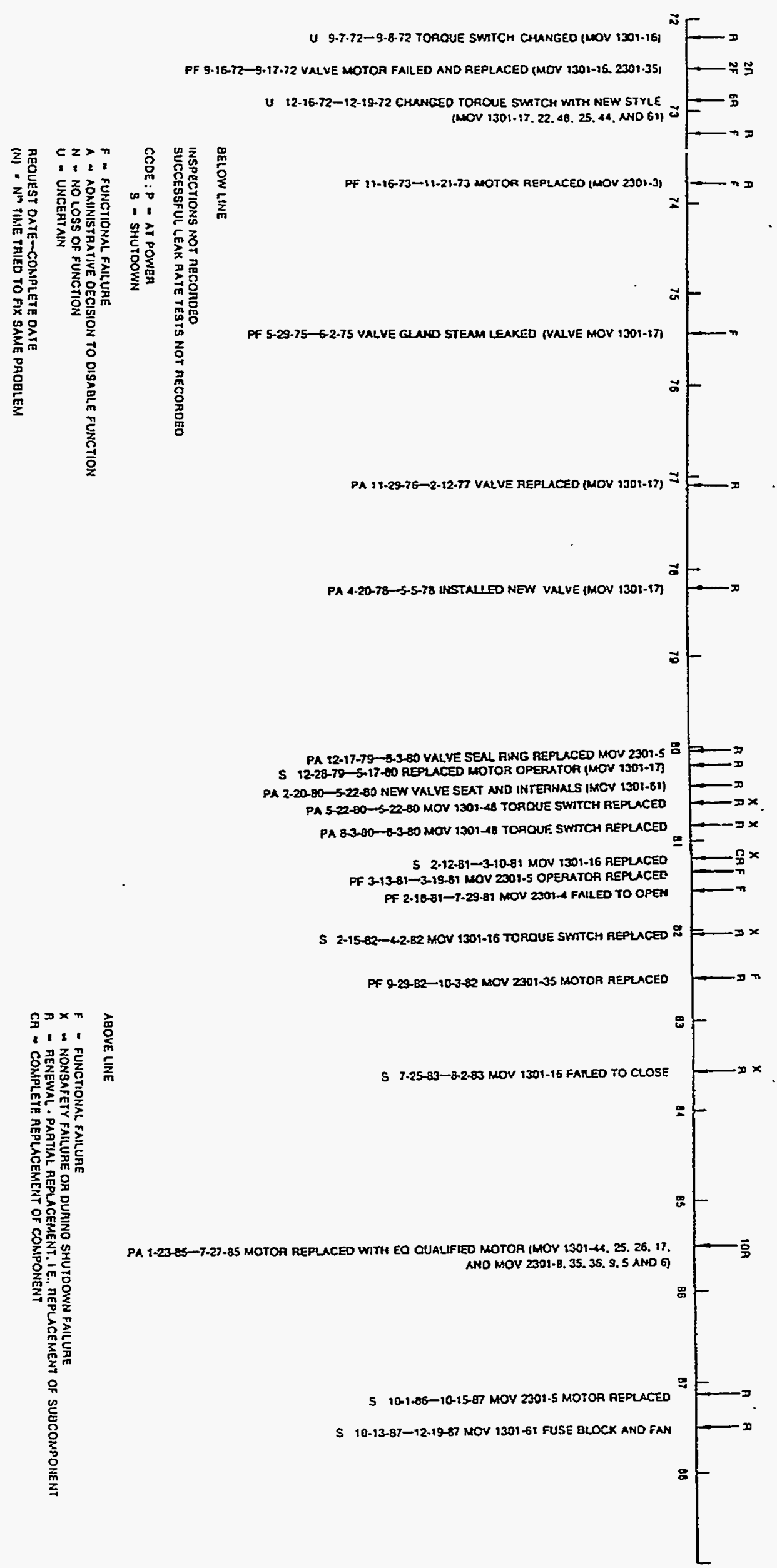




\subsection{Data Used in Aging Assessments for Passive Components}

Generic data bases that reflect operating experience do not contain much information on passive components, nor do current PRA studies address the aging failure of passive components. As part of the NPAR program, a method using probabilistic structural analysis has been developed in the Aging Risk of Passive Components Project that incorporates the effects of passive component aging in a PRA (Phillips et al., 1992). One objective of this method is to prioritize the passive components for the plant inspection program. When the most significant passive components are identified and the aging failure rates are estimated, the PRA can be modified to incorporate the passive components in estimating plant risk.

Instead of using data bases that record the events of component failures as in the TIRGALEX approach, probabilistic structural analysis techniques calculate the failure probabilities (Fig. 3.4) from stress and strength distributions. Aging causes the spread and shifting of stress and strength distributions and causes an increase in the probability of failure. Physical models described in Sec. 2 of this report are some of the candidates for models that were used in the probabilistic structural analysis.

A modified PRAISE (Piping Reliability Analysis Including Seismic Events) model was chosen in the Aging Risk of Passive Component Project at INEL for the purpose of demonstration. In this analysis, the weld of the piping section on the downstream side of the cycling check valve of the auxiliary feedwater system was examined to demonstrate how probabilistic structural analysis could be used to estimate the probability of component failure because of aging. It is expected that the weld at this location will be exposed to stress environments occurring during normal plant transients (e.g., heatup, cool-down, and scrams). PRAISE is a probabilistic fracture-mechanics code that models the growth of piping flaws affected by anticipated or accidental stresses during the operation of a nuclear power plant. When these flaws grow to critical lengths, they cause the pipe to leak or catastrophically break. PRAISE was modified in this project to account for the aging effects. Figure 2.1 shows the basic concept of the PRAISE code.

The information that is required in fracture mechanics analyses varies because the failure mechanisms that are modeled are different. For example, as shown in Fig. 2.1, the PRAISE model needs the initial crack-size distribution, the nondetection probability, information on material properties' (such as material toughness or S-N curve) stress history, cyclic stress history, crack growth history, the probability of leak before break, and failure criterion. The results of the INEL analysis indicate that knowledge about the input parameters that most contribute to failure caused by a particular mechanism (fatigue, erosion/corrosion, etc.) would be useful in the control of aging. However, the analysts acknowledged that material property data on aging components, especially data associated with plants that have more than $40 \mathrm{yr}$ of operating experience, are not easily available for the analyses. In fact, the material property data used in the INEL analysis are based largely on expert opinion. The confidence of the conclusions reached through this type of analysis is highly sensitive to the accuracy of the data that are available to the analysts.

Part of the effort of the NPAR comprehensive studies (Phase II) is to understand the material properties that are associated with various aging mechanisms of selected components at nuclear power plants and therefore are in the same general area of the probabilistic structural analysis. Data gathered in these studies include parameters that can be used as indicators of the degrees of aging, the residual life, and component reliability. Table 2-1 lists the degradation mechanisms on major PWR components studied in the NPAR program (Shah et al., 1989; Jaske and Shah, 1990).

Physical phenomena associated with aging mechanisms are complex; however, they are generally amenable to mechanistic modeling. The current practice is to adopt one of the following two approaches. 


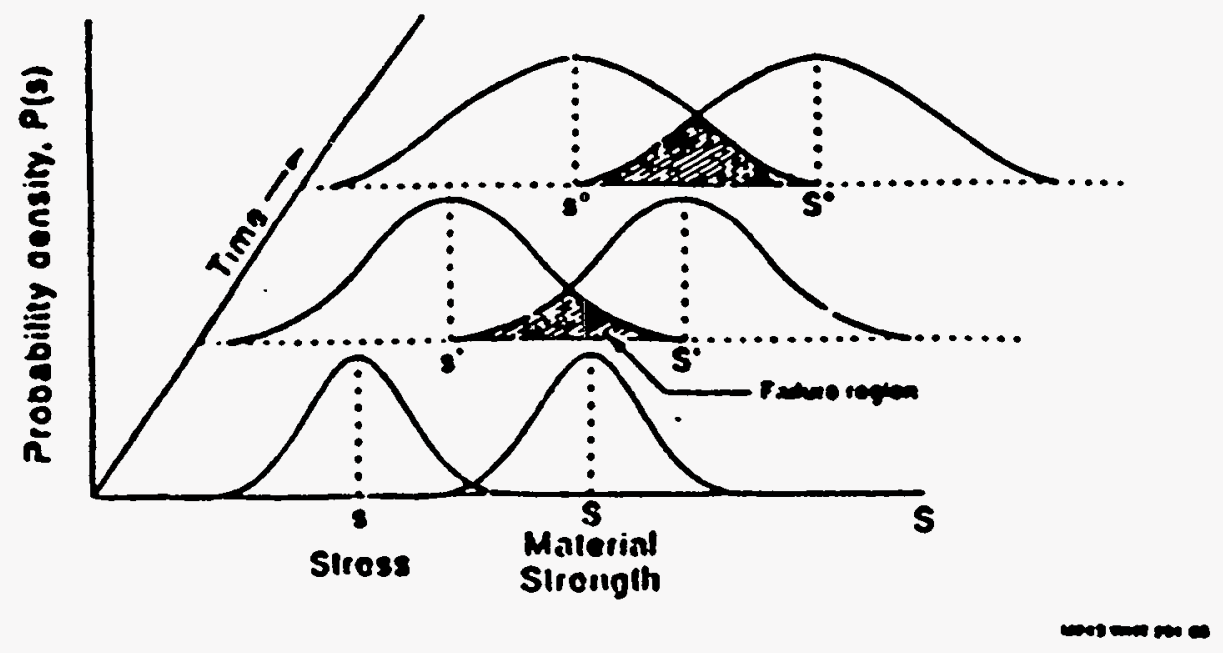

Figure 3.4 Stress-strength interference (SSI) increases with plant age (Phillip et al., 1992) 
(1) Empirical models. An extensive data base is gathered on the phenomenon under all credible circumstances and a procedure is developed for interpolating this data base. In the empirical approach, parameters that require the models to be extrapolated outside the operational environment are not considered reliable.

(2) Phenomenological models. The physical phenomena of a specific aging mechanism are modeled with a set of assumptions that often greatly simplify the physical situation. Although reality is simplified, these models can be correlated with the available laboratory and field experience and are more reliable when it is necessary to extrapolate the data to accommodate situations that are beyond the normal operating environment.

In the following sections, we discuss briefly some empirical models and phenomenological models for identified aging mechanisms and the data available for these analyses. The intent of the discussion is not to review the technical details of the models; rather, it is to indicate the kind of data needed in physical reliability models and the availability of these data.

\subsubsection{Empirical Models}

One well-known empirical formulation for piping and vessel reliability is from $\mathrm{H}$. M. Thomas (1981). In that paper, Thomas shows that the reliability of pressure vessels can be estimated based on an analysis of actual failure statistics. In his approach, the probability of catastrophic failure, $P_{C}$, is considered to be a subset of the more general leakage probability, $P_{L}$. To find $P_{C}$, the ratio $P_{C} / P_{L}$ is first estimated from fracture mechanics, and the probability, $\mathrm{P}_{\mathrm{L}}$, is estimated from global vessel statistics and modified by specific plant factors as well as wear and deterioration factors. The estimate of $\mathrm{P}_{L}$ is given by the following formula:

$$
P_{L} \propto Q_{e} \times F \times B \times q \times w
$$

where

$$
\begin{aligned}
Q_{e} & =\text { equivalent size, shape, and weldment factor; } \\
F & =\text { age factor; } \\
B & =\text { learning curve; } \\
q & =\text { quality difference factor; and } \\
\text { w } & =\text { sum of all wear factors (fatigue, corrosion/erosion, etc.). }
\end{aligned}
$$

In the above equation, it is argued that for pressure vessels, the most powerful factor of all is the $Q_{e}$ factor, which is estimated on the basis of the weak link theory. The equivalent factor $Q_{e}$ is determined to be the sum of the parent material, $Q_{p}$, and 50 times the weld zone, $Q_{w}$, i. e. $Q_{e}=Q_{p}+$ $50 \mathrm{Q}_{\mathrm{w}}$. It is assumed that the annual leakage rate is independent of age, and is given by $\mathrm{P}_{\mathrm{L}}=10^{-8} \mathrm{Qe}^{-1}$. To estimate this simple leakage rate, Thomas considered the flowing sources of data.

(1) WASH 1318: Technical Report on Analysis of Pressure Vessel Statistics from Fossil-Fueled Power Plants

(2) ASME I and ASME VIII vessels 
(3) EPRI pipe failure statistics

(4) Steam generator tube failures

(5) Oil pipelines (W. Europe)

(6) Transmission gas pipelines (USA)

(7) Interstate gas pipelines (USA)

The approximation hierarchy is based on these data sets, which generally show that the failure rates of pipes and vessels decrease with time. Therefore, it is argued that the age factor, $F$, can be estimated from the presence of congenital defects, but generally speaking, give a decreasing failure rate beyond about $10 \mathrm{yr}$. The learning curve factor, B, is also an improvement. Finally, his analysis of wear factors, such as fatigue and erosion/corrosion, shows that they do not contribute in a significant way during the first two decades of the pressure vessel's lifetime. This is shown in Fig. 3.5.

Thomas's analysis shows that the dominant component of the bathtub curve during the first $40 \mathrm{yr}$ is the congenital defect (burn-in) period as shown in Fig. 3.6. He argues that the many theoretical models on fatigue and crack growth in the literature are in conflict with the observed failure statistics. However, the probabilistic fracture mechanics analysis performed at INEL (mentioned earlier) also shows a failure rate that decreases with time.

It should be pointed out that this reasoning does not apply to reactor pressure vessels because the material properties are degrading with time, which causes the effect of the "accelerating wear factors" of Fig. 3.6 to be felt earlier.

\subsubsection{Phenomenological Models}

\subsubsection{Fatigue}

Fatigue is an important deterioration process for components, which include the RPV, piping, BWR metal containment, RCP pump casing, reactor internals, etc. The basic methods of deterministic fatigue analysis include the cycles-to-failure models and crack propagation laws. The cycles-tofailure models provide estimates of the number of stress cycles it takes for the material to fail. Models in this category include the S- $\mathrm{N}$ curve for regular cyclic-loading conditions, the Palmgren-Miner rule for irregular cyclic-loading conditions, and some modified versions of these two models. Crack propagation laws (such as the Paris law) relate the crack growth rate to the level of cyclic stress.

$\mathrm{S} / \mathrm{N}$ curves, Palmgren-Miner's rule, or other cycles-to-failure models require a great deal of detailed bookkeeping of the history of the complex loading and service environment. Although the numbers of cycles to failure under different stress levels can be obtained from test data that simulate the service environment, the collection of these data can be very difficult and time-consuming in an operating power-plant setting. On the other hand, crack propagation laws require data generated experimentally in environments and frequencies that are as realistic as possible and over a wide spectrum of growth rates. Sufficient data must be obtained to achieve reasonable confidence in the resulting correlation. The simulation of the operating environment and the large number of tests needed can present problems in correlating the crack-size growth with stress intensity factors. Furthermore, linear extrapolation of the results from accelerated tests may lead to overconservative conclusions and underestimate the component residual life (Yahr et al., 1984). 


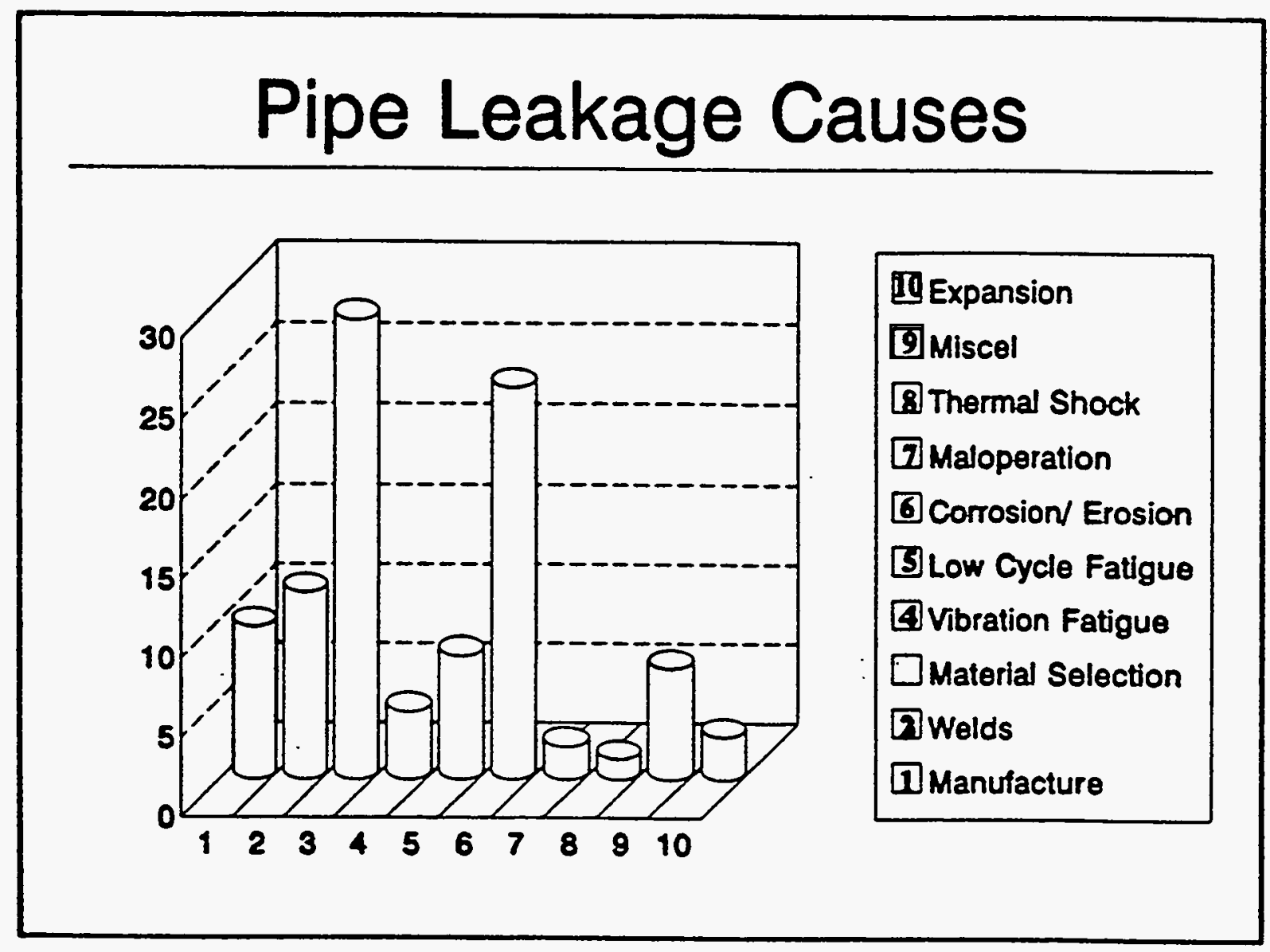

Figure 3.5 Pipe leakage causes by percentage 


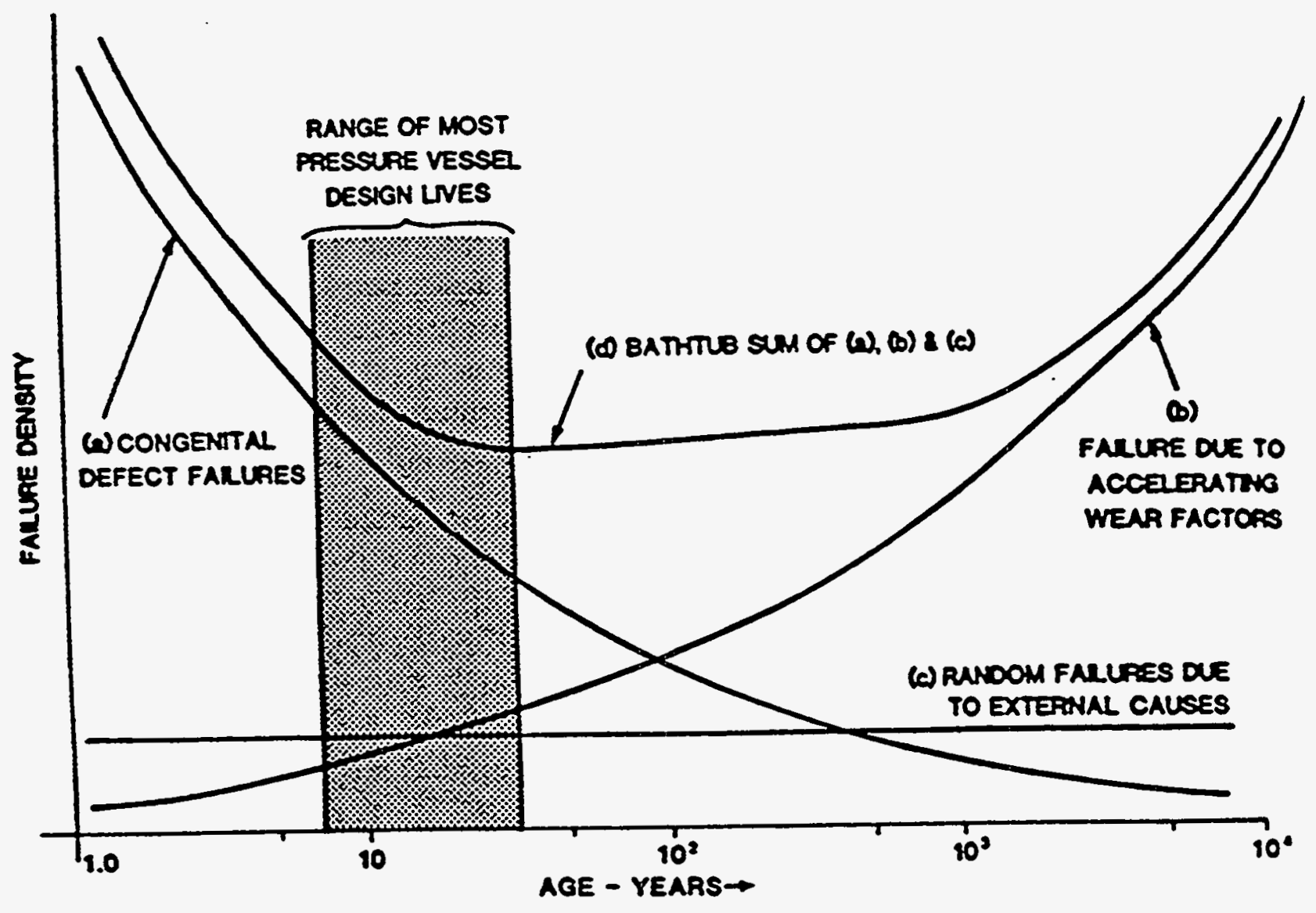

Figure 3.6 The Bathtub Curve related to pressure vessels (Thomas, 1981) 
Fatigue Data and Design Rules for Ferritic Steels and Welds

The RPV and piping components are manufactured of carbon and low-alloy steels. In the following, we summarize the data and design procedures for these steels and their weldments. This data and design rules can be found in references Manjoine and Johnson (1986), Oliver and Ritter (1979), Harrison and Maddox (1980), The Welding Institute (1982), Gurney (1976), Manjoine and Gowda, and the ASME Boiler and Pressure Vessel Code (ASME, 1983a, 1983b). However, the rules for welded joints are not that detailed and are still an a state of development (Manjoine and Gowda).

In Manjoine and Gowda, a number of design rules are proposed for fatigue design of ferritic steels and their weldments up to temperatures of $700^{\circ} \mathrm{F}$. These are summarized below.

\section{For Base Metal within Three Thicknesses of Weld}

The quantity is the higher of the following.

Stress Range: $\Delta S=1200 \mathrm{~N}^{-0.3}$ or $19.1 \mathrm{~N}^{-0.01}$

Number of Cycles: $N=(\Delta S / 1200)^{-3.33}$ or $(\Delta S / 19.1)^{-100}$

For other weldment configurations,

$$
\Delta \mathrm{S}_{\mathrm{i}}=\mathrm{C}_{\mathrm{i}} \Delta \mathrm{S},
$$

where $C_{i}$ is a reduction factor for a given class of weldments and varies between 0.33 and 1.0.

For the Base Metal Outside the 3t Distance

Alternating stress range, $\Delta S=2 E\left(\varepsilon_{p d}+\varepsilon_{e d}\right)$, where

$$
\varepsilon_{\mathrm{pd}}=0.0788 \ln \left(\frac{100}{22+0.15 \mathrm{UTS}}\right)(2 \mathrm{~N})^{-[0.45+0.001 \mathrm{UTS}]}
$$

for no applied mean stress and

$$
\varepsilon_{\mathrm{pd}}=\text { higher of } \frac{\mathrm{UTS}+40}{6 \times 10^{4}}(2 \mathrm{~N})^{-0.09} \text { or } \frac{0.35 \mathrm{UTS}+9}{4.85 \times 10^{4}}(2 \mathrm{~N})^{-0.009}
$$

for maximum applied mean stress.

$$
\varepsilon_{\text {ed }}=\text { higher of } \frac{U T S+40}{6 \times 10^{4}}(2 N)^{-0.09} \text { or } \frac{0.35 U T S+9}{7.28 \times 10^{4}}(2 N)^{-0.009}
$$

where $\mathrm{N}$ is the number of cycles, $\Delta \mathrm{S}$ is in $\mathrm{K}_{\mathrm{si}}$, UTS is the ultimate tensile strength, and $\mathrm{E}$ is the modulus at temperature.

The design equations given above compare well with extensive data presented in Oliver and Ritter (1979) and Harrison and Maddox (1980). Over the range of cycles from $10^{3}$ to $10^{6}$, the reduction factor of the design equations given above and the mean values of the data is about a factor of 2 . The standard deviation is about 0.6 of the mean stress, and the endurance limit is found to be achieved between $10^{5}$ and $10^{6}$ cycles. 


\subsubsection{Irradiation and Thermal Embrittlement}

The major effects of irradiation and thermal embrittlement are the decrease in ductility, the increase in the ductile-to-brittle transition temperature (DBTT), and the decrease of the USE. The degree of material embrittlement is most often evaluated by CVN impact energy tests (Flinn and Trojan 1990) and fracture toughness tests.

The CVN test involves a specimen of the test material in the shape of a square bar with a V notch. The material is struck by a calibrated swinging arm, and the energy absorbed is measured. Empirical correlations were derived for the increase in the transition temperature and the neutron fluence (Odette and Griesbach, 1987; Guthrie, 1983) and between DBTT and thermal aging (Chopra, 1990). Most of the uncertainty in these estimates was caused by the uncertainty in the correlation models. Estimating the cumulative neutron fluence (in radiation-related aging) and thermal activation energy (in thermal aging) that components are actually being exposed to throughout the years also introduces difficulty and uncertainty.

Fracture toughness is the property that indicates the susceptibility of the material to the spread of a crack. As the embrittlement process progresses, it is necessary to relate the shifts in the DBTT and in the USE to the fracture toughness. At lower temperatures, i.e., in the brittle regime, the linear-elastic crack-initiation toughness, $\mathrm{K}_{\mathrm{Ic}}$, and the crack-arrest toughness, $\mathrm{K}_{\mathrm{Ia}}$, are used to determine the fracture. However, at higher temperature, elastic-plastic parameters for characterizing the toughness are used. Stable crack-growth resistance curves (J-R curves), are used to characterize the upper-shelf toughness, JC, and the CVN energy (Hiser, 1987).

To achieve reasonable confidence, both the approaches (CVN impact energy tests and the fracture toughness tests) require a sufficient amount of sample data to derive reliable correlations. The correlations also may be sensitive to the composition of the material and the operating environment.

\section{Data and Design Rules for Radiation Embrittlement of Pressure Vessels}

Recent studies in the US have focused on four different areas of investigation: (1) demonstration of tests of improved steel production, (2) variable radiation sensitivity, (3) post irradiation heat treatment for embrittlement relief, and (4) correlation of fracture toughness with irradiation. In the following, we summarize the data base and some of the salient results of these studies.

US supplemental specifications for improving the irradiation sensitivity of steels and welds place greater restrictions on allowable contents of copper, phosphorous, sulfur, and vanadium impurities. Restrictions on copper and phosphorous content are intended to improve radiation resistance, whereas those on sulfur and vanadium are intended to elevate the USE to provide a greater toughness reserve against radiation degradation. The merit of those supplemental specifications has been firmly established experimentally (Hawthorne et al., 1979a). Comparisons of the radiation resistance of pressure vessel steels and welds produced in Germany, France, and Japan $(0.01$ to $0.07 \% \mathrm{Cu})$ with the results for improved US steels $(0.1 \% \mathrm{Cu} \max )$ show good agreement (IAEA, 1977). The data trends show a significant improvement in the radiation sensitivity of steels with copper contents less than 0.1\% (IAEA, 1977; Hawthorne, 1982).

One study by Naval Research Laboratory (NRL) focused on the effects on nickel alloying and copper impurities on the radiation sensitivity of copper (Hawthorne 1979c). Other comparisons were made for A533-B steels $(0.4$ to $0.7 \% \mathrm{Ni}$ ) and A302-B steel ( $0.4 \% \mathrm{Ni}$ ) (Palme, 1973). Additional investigations for the NRC explored the effects of combined copper, nickel, and phosphorus contents, as well as the combined effects of impurities and alloying elements on the radiation sensitivity (Hawthorne, 1981). At the same time, two reviews of data banks on irradiated steels and welds were made in the US. The Metal Properties Council compiled test reactor and power reactor irradiation data as of 
November 1977 (Metal Properties Council, 1983). Combustion Engineering (CE) initiated another effort for EPRI in late 1979. These studies were continuations of earlier efforts by two CE investigators [Varsik and Byrne (1979)]. The transition temperature relationship developed by their investigation is

$$
\left.\Delta \mathrm{NDTT}_{\text {norm }}=\mathrm{F} \text { (chemistry ratio } \times \mathrm{Cu}\right) \text {, }
$$

where $\triangle$ NDTT is the transition temperature normalized to a fluence of $3 \times 10^{19} \mathrm{n} / \mathrm{cm}^{2}$ and where the chemistry ratio is the value of

$$
\text { atomic percent }\left[\frac{1.5 \mathrm{Ni}+\mathrm{Si}+0.5 \mathrm{C}-0.5(\mathrm{Mn}-0.5)}{0.5+0.5 \mathrm{Mo}}\right] \times \mathrm{Cu} \text {. }
$$

This approach modified the general equation for transition temperature increase given by NRC Regulatory Guide 1.99:

$$
\Delta \mathrm{rT} \mathrm{NDT}=[40+1000(\% \mathrm{Cu}-0.08)+5000(\% \mathrm{P}-0.008)]\left[\frac{\Phi}{10^{19}}\right]^{0.5}
$$

The possibility of a saturation of radiation embrittlement was raised by Westinghouse (Yanichdo and Chirigos, 1980), and experimental programs to resolve this question were set up by EPRI and NRC.

Post-irradiation heat treatment (annealing) is a method used for the periodic reduction in the amount of accumulated embrittlement. The method offers one possible solution to the high embrittlement levels for high-copper-content welds and has been studied extensively by NRL for the NRC (Hawthorne et al., 1982; Loss et al., 1982) and by Westinghouse for EPRI (Mager, 1978). The data trends with annealing and reirradiation vs the trends without annealing verify that the method can be very effective in reducing the build-up of radiation effects. Experimental correlations also were established between the transition temperature indexed by the 41-j temperature and that by the fracture toughness KJ 100 MPa.m $^{1 / 2}$ (Hawthorne, 1979b, 1979c). Excellent correlations were found, with temperature uncertainty of $\pm 15^{\circ} \mathrm{C}$.

\subsubsection{Erosion/Corrosion}

Erosion/corrosion presents a significant aging concern at nuclear power plants. A particular problem area is the wall thinning of the carbon steel piping. The existing empirical models (Chexal and Horowits, 1989; Henzel et al., 1988) show that the wall thinning rates because of corrosion/erosion are functions of the following factors: (1) piping material, especially the $\mathrm{Cr}$ and Mo content $(\mathrm{h}=\mathrm{Cr}+$ Mo \%); (2) fluid velocity ( $w$, in $\mathrm{m} / \mathrm{s}$ ); (3) piping geometry (with a geometric factor $\mathrm{k}_{\mathrm{c}}$ ); (4) dissolved oxygen concentration ( $\mathrm{g}$, in $\mu \mathrm{g} / \mathrm{kg}$ ); (5) water chemistry ( $\mathrm{pH}$ value); and (6) water temperature ( $\mathrm{T}$, in $\left.{ }^{\circ} \mathrm{K}\right)$. Extrapolations of the thinning rate beyond the applicable range are not recommended. Typical results of such correlations are shown in Figs. 3.7 and 3.8 .

\subsubsection{Probabilistic Fracture Mechanics}

Because both stress and strength in the physical models that describe the aging phenomena are random in nature, the failure of material can be estimated probabilistically if the parameters are treated as random variables. Component reliability models have been developed that were based on fracture mechanics models. For example, Chen (1991a and 1991b) described techniques for estimating of the fatigue reliability at a single stress level and at varied stress levels based on the S-N curve and the Palmgren-Miner rule. 

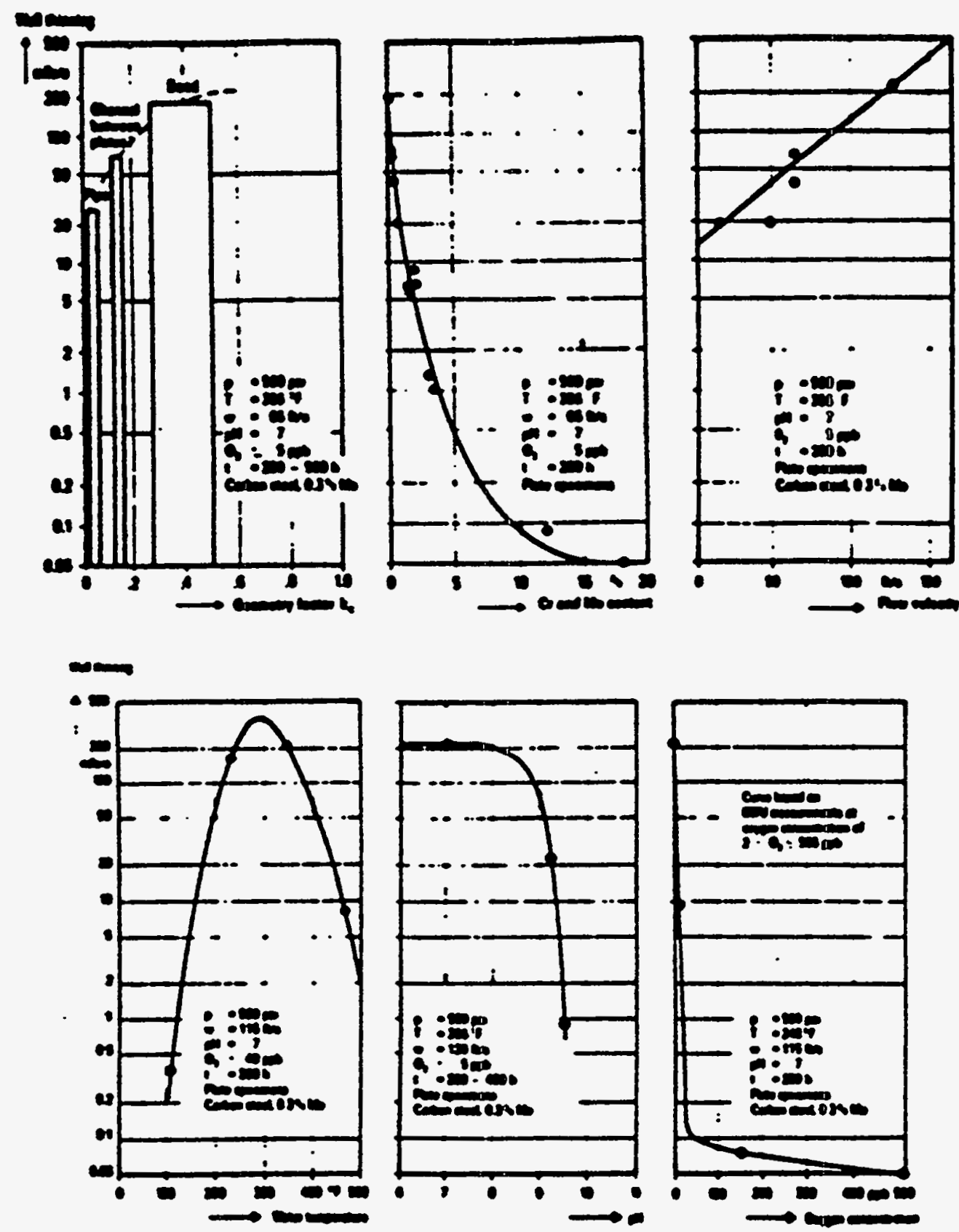

Figure 3.7 Effect of $k_{c}, h, w, T, p H$, and $\mathrm{O}_{2}$ on pipe wall thinning 


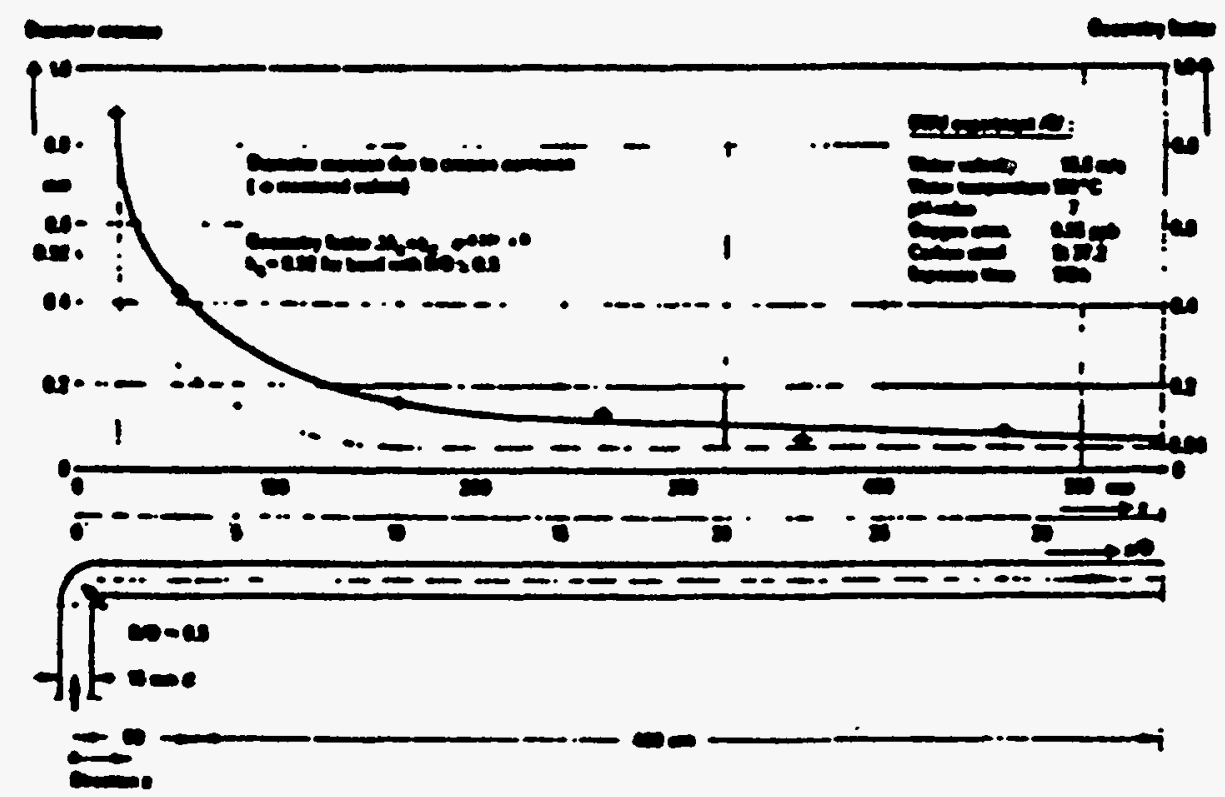

Figure 3.8 Pipe diameter increase as a result of corrosion 
Approaches for estimating the fatigue reliability also have been proposed that are based on three basic types of models on probabilistic crack growth: crack-growth low-randomization models (Lin et al., 1984; Ortiz and Kiremidjian, 1988), the evolutionary probabilistic models using the Markov or diffusion approach (Sobczyk and Spencer, 1992), and cumulative jump models (Ditlevsen and Sobczyk, 1986). To estimate the fatigue reliability using a probabilistic crack-growth model, the distribution of initial crack size must be specified, the stress history must be assumed, and the failure criterion must be formulated. Assessing these data often involves a significant amount of field and laboratory datacollection effort and often the elicitation of expert opinion, but still the uncertainty of these data is large. The example using the PRAISE model to estimate the failure probability of piping, mentioned earlier in this section, is one such application. Other examples are the estimation of the failure probability of the vessel (Becher and Pedersen, 1974) and of the piping (Harris and Lim,1982).

Similar concerns over the availability and quality of data are also applicable to other probabilistic structural and fracture mechanics models.

\subsection{Review of Statistical Methods Used in Aging Data Analysis}

This section examines current statistical methods used to describe and evaluate the effects of aging on machine and component lifetimes, and alternatives to the existing procedures are discussed. We will focus specifically on parametric modeling procedures implemented in Wolford et al. (1992), which incorporate previous statistical aging studies by Vesely (1987) and Salvia (1980). To evaluate the effects of aging on components, Wolford et al. (1992) construct parametric failure rate models (PFRMs) that include numerous assumptions about the underlying failure time distribution of the components in the study. Assumptions like the ones made in that report are often necessary to conclusively answer specific questions about the effects of aging on component reliability. The models are described fully in Chap. 2 of that NUREG report and will be described only briefly here. Overall, the methods are thoroughly and carefully derived and should be more than sufficient for modeling aging effects if applied correctly. The step-by-step approach outlined in that report not only allows the possible identification of component aging but also the assessment and description of the aging process on the components' stochastic lifetime. Given that we satisfy the models' assumptions and data requirements, the prescribed analyses are certain to be satisfactory for inclusion in a plant PRA report.

\subsubsection{Parametric Failure Rate Models}

The assumptions and data needs are critical in the aging analyses. Consider the parametric forms for the failure rates proposed for the PFRMs.

$$
\begin{aligned}
& \lambda(t)=\lambda_{0} e^{\beta t} \text { (exponential failure rate) } \\
& \lambda(t)=\lambda_{0}\left(\frac{t}{t_{0}}\right)^{\beta} \text { (Weibull failure rate) } \\
& \lambda(t)=\lambda_{0}(1+\beta t) \text { (linear failure rate) }
\end{aligned}
$$

Each model has historic roots in machine and material lifetime analyses, the Weibull failure rate (WFR) being the most commonly used of the three. The linear failure rate (LFR), simplest in form, has been used in determining age-dependent risk (Vesely, 1992, 1988, 1987; Vesely et al., 1990), and some serious concerns about the use of LFR in aging analyses were raised in Chap. 2 of this report. Some advantages and disadvantages from choosing one model over another are discussed in Wolford et al. 
(1992). Empirically, they observed that the LFR performed poorly in more instances than the other two models and, because of its nonexponential form, was most difficult to work with analytically.

Although these failure curves may suit certain aging mechanisms very well, there may be very little evidence that an explicit physical relationship exists between these models and the specific effects of the aging with which we are concerned (fatigue, irradiation embrittlement, and corrosion). As a consequence, we are fitting these parametric models to the component lifetime distributions mostly by suggestion from the data. If we have a sample of recorded lifetimes that is large enough and the parametric constraints do not greatly contradict the true underlying lifetime distribution, the data will most likely fit the model adequately. However, as can be seen throughout the literature, failure data pertaining to component aging are scarce and justification for parametric models is sometimes dubious.

It is not certain exactly how much data constitutes "enough." The analyses for the PFRMs use normal approximations in parameter estimates that typically require 20 to 30 (or more) samples before such inferences can be considered accurate. Mann, Schafer, and Singpurwalla (1977) suggest larger samples of 100 or more before maximum likelihood estimates based on Weibull lifetimes appear normally distributed. It is not clear how well these approximations do with smaller samples.

To illustrate how sample size effects the accuracy of inference and testing with the PFRMs proposed, the parametric WFR model will be used. Estimation of the Weibull parameters is difficult because the maximum likelihood estimators (MLEs) cannot be formulated explicitly. The probability density function for the Weibull model is

$$
f(t)=\lambda_{0}\left(\frac{t}{t_{0}}\right)^{\beta} e^{-\lambda_{0}\left(\frac{t_{0}}{\beta+1}\right)\left(\frac{t}{t_{0}}\right)^{\beta+1}}
$$

with corresponding failure rate expressed in Eq. (3.6).

The Weibull model is directly related to the WFR model, similar to the exponential failure rate (EFR) model, and has a linear failure rate as a special case, hence deductions based on the Weibull model pertain to the PFRMs as well.

For estimation of the shape parameter $(\delta)$ in the WFR model, Mann, Schafer and Singpurwalla (1977) suggest samples of 100 or more before maximum likelihood estimates of Weibull parameters appear normally distributed. In fact, good approximate tests and confidence intervals can be obtained with somewhat smaller samples. Figure 3.9 shows how the distribution of the shape parameter estimate appears normal (Gaussian) when $n$ is larger than 50.

Among the three proposed models, the EFR and the WFR are more alike than the LFR. The WFR and EFR are more flexible for modeling the failure rate, and the EFR seems to cause the fewest problems in the inference calculations. Because of the rigidness of the parametric modeling, none of the PFRMs should be trusted for extrapolations much beyond the latest failure time in the data. Also, none of the three failure rates do well at accommodating discrepancies like the existence of an underlying bathtub failure rate or extended burn-in period. By design, all three models are constructed to allow a basic test for positive slope in the failure rate. This test is an essential step in the failure rate inference because further modeling presupposes that the failure rate is not constant.

There are other problems encountered with this type of parametric procedure. It is pointed out in the report that the many assumptions about component lifetime distributions are not believed to be perfectly true, and it was actually noted that assumptions of independent Poisson occurrences (which are essential to the model) are suspect after viewing obvious non-Poisson clusters within the available data. 


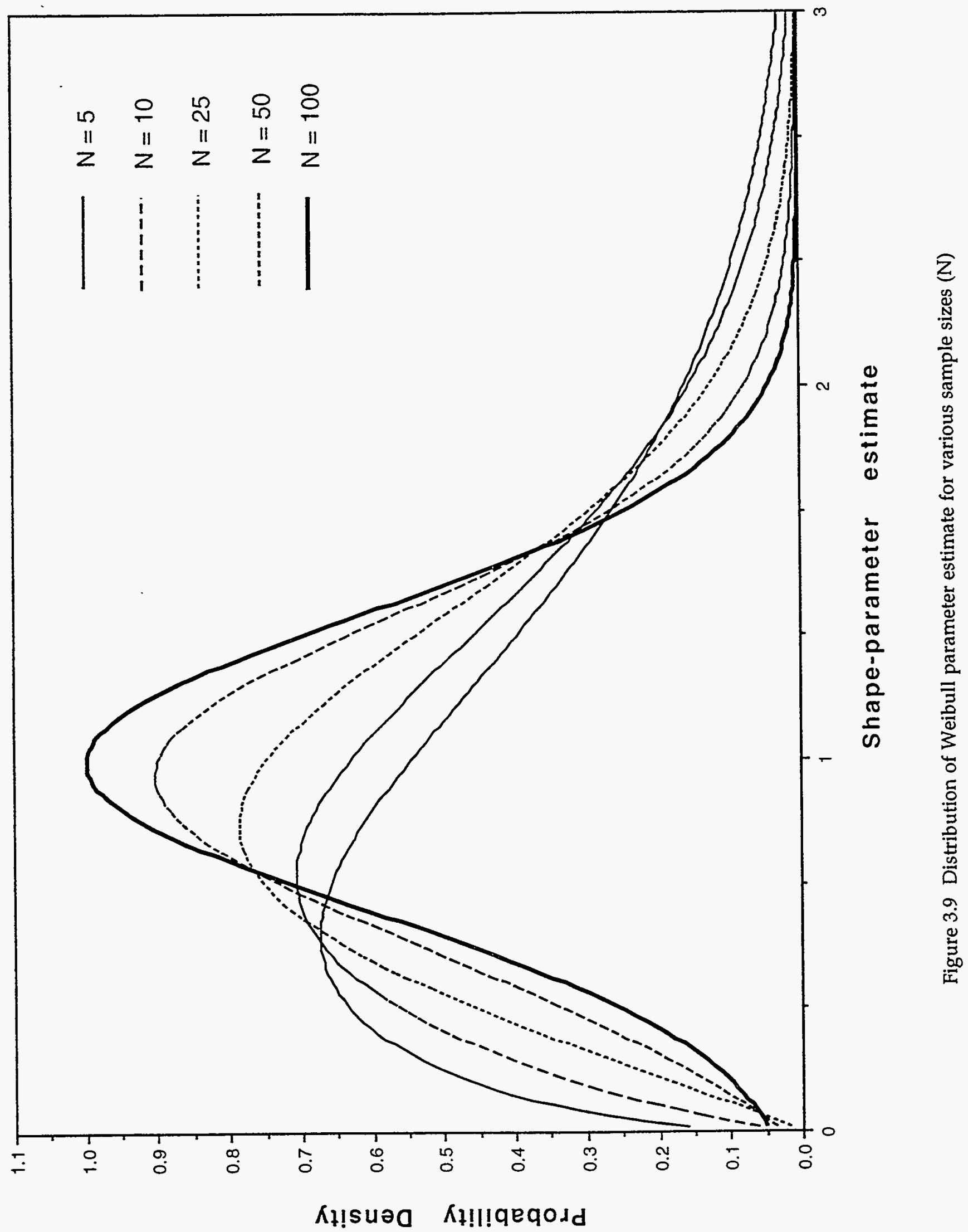


However, the greatest problem with the statistical contribution to aging studies is not the inadequacy of the statistical models but the lack of data available to test such models. In a modern statistical analysis, it is not uncommon to sidestep the problem of insufficient data by artificially improving the study by using assumptions that may not be realistic. Constraining lifetimes in the study to fit parametric models may simplify the analysis. Such models are rarely rejected if small data sets are used. Normal approximation for parameter estimates based on small samples also may bolster the range of inference while possibly defeating the integrity of the study. If the data are too scarce to allow separate analysis of the components and the associated plant-to-plant variability, component groups often are pooled for mathematical convenience not necessarily because of a strong belief of homogeneity between the pooled groups.

Using these kinds of shortcuts will cast doubt on the significance of the final results, so assumptions leading to oversimplification should be scrutinized closely by those who are responsible for the validity of the study's conclusion. The quality of the data also should be scrutinized closely. Specifically, the use of "broadly defined" failures dubbed in Wolford et al. (1992) should be defended before we draw conclusions based on them. Although the analogous narrowly defined failures are approached with certainty in the report, broadly defined failures seem less assured and their validity is not clear. However, we note that the domain of inference using only the narrowly defined failures is minute in comparison to the existing analysis, which uses much more data.

If the amount of data seems insufficient to answer the series of questions we have about the aging effects for the various components, we should consider simplifying the questions rather than oversimplifying the analysis with possibly invalid assumptions. Given a set of components that work independently and have the same underlying probability distribution, we should first find out if the single failure rate associated with the component lifetime significantly increases over time or stays about the same. This question is posed in Wolford et al. (1992) but under the guise of an assumed failure rate model. As a consequence, unless the model perfectly suits the underlying failure rate for the examined components, the answer we obtain may not be directly applicable to the question we asked. A nonparametric test of failure rate should first be imposed on the failure data. There are a number of nonparametric inference-based procedures available, and we will discuss three basic tests that are relevant for lifetime data samples for which we are most interested.

\subsubsection{Test for Increased Failure Rate}

The first test is one for increased failure rate (IFR). An IFR life distribution, as defined in Barlow and Proschan (1981), is simply one in which the failure rate exists and is nondecreasing. A total-time-on-test transform is used by Klefsjo (1980) to derive a hypothesis test for IFR vS a nullhypothesis that the underlying failure rate is constant (and hence the underlying distribution is exponential). The test statistic, based on normalized spacings of the ordered lifetimes, is cumbersome for medium to large samples. We define

$$
D_{i}=(n-i+1)\left[X_{(i)}-X_{(i-1)}\right]
$$

and

$$
\alpha_{i}=\frac{(n+1)^{3} i-3(n+1)^{2} i^{2}+2(n+1) i^{3}}{6}
$$

where $X_{(1)}, \ldots, X_{(n)}$ denotes the observations in ranked order from smallest to largest. The nullhypothesis can be rejected (in favor of the hypothesis that the lifetimes have IFR) if the test statistic 


$$
A=\frac{\sum_{i=1}^{n} \alpha_{i} D_{i}}{\sum_{i=1}^{n} X_{i}}
$$

is significantly small. A table of tail values for the distribution of $A$ (under the null hypothesis) is found in Klefsjo (1983). Klefsjo also proposes the transformation

$$
\mathrm{A}^{*}=\frac{86.94 \mathrm{~A}}{\mathrm{n}^{3.5}},
$$

which can be treated as a standard normal random variable. This class of lifetime distributions is narrowly defined because it does not include membership of component lifetimes that may show slight improvement in failure rate periodically (perhaps because of improved maintenance) while showing an overall aging effect.

To illustrate its use, we applied the IFR test to some of the component data from Wolford et al. (1992). However, failure times that we used for various pumps and valves were interpolated from graphs and may not reflect the true data. Also, the sampling method is not perfectly clear to the reader, so a perfect-repair model is assumed, which means the recorded failures represent the entire lot of available data. The results of the nonparametric IFR tests appear to be in disagreement in some instances, but since the different tests measure slightly different qualities of the data, varying test results need not be contradictory.

Differences in conclusions also are produced by the effective power of the statistical tests. For small samples, type I error for the nonparametric IFR test may be underestimated (see Fig. 3.10, discussed later), hence if the test is adjusted to be unbiased, the power is reduced. However, if the data violate the assumptions of the parametric procedure, the power of the corresponding test can be overestimated. We note that from the one large sample of motor operated valves, summarized in Table 3-3, the tests are not in disagreement.

\subsubsection{Test for Increased Failure Rate Average}

A test for increasing failure rate average (IFRA) is slightly more general and more inclusive than the IFR test. A distribution function (F) has an IFRA if its hazard function, defined as the accumulated failure rate, increases linearly (or faster) in time. Specifically, if the failure rate is denoted $\lambda(t)$, then $F$ is IFRA if

$$
\frac{1}{t} \int_{0}^{t} \lambda(x) d x
$$

increases for all $t>0$. If an operating component accumulates damage and effects from aging via shocks that are generated from a Poisson process and the component fails when the damage from the shocks exceeds a fixed threshold value, that component's lifetime will be IFRA. Note that all IFR distributions are IFRA but not all IFRA distributions are IFR.

The test for IFRA is similar to the IFR test mentioned above. We start with the IFR test and replace $\alpha_{1, \ldots, \alpha_{n}}$ with 


$$
\beta_{i}=\frac{2 i^{3}-3 i^{2}+i\left(1-3 n-3 n^{2}\right)+2 n+3 n^{2}+n^{3}}{6}
$$

for $\mathrm{i}=1, \ldots, \mathrm{n}$. Again, the null-hypothesis can be rejected (in favor of the hypothesis that the lifetimes have IFRA) if the test statistic

$$
B=\frac{\sum_{i=1}^{n} \beta_{i} D_{i}}{\sum_{i=1}^{n} X_{i}}
$$

is significantly small. Table values for a test of hypothesis are listed in Klefsjo (1983), where it is shown that the test statistic

$$
\mathrm{B}^{*}=\frac{\mathrm{B} \sqrt{210}}{\mathrm{n}^{2.5}}
$$

is an asymptotically standard normal random variable. Thus, standard normal tables also may be used for this IFRA test.

\subsubsection{Test for "New Better than Used"}

As an alternative to the IFR test, the "New Better than Used" (NBU) test is used to detect distributions of component lifetimes in which existing lifetimes at any fixed age have a stochastically smaller residual life than that of a new component because of some deterioration process like aging. The class of IFRA distributions actually is contained in the NBU class (not vice versa), but the differences are subtle. Consider a component that ages negligibly except for distinct points in time when shocks occur to the component. If the probability of surviving a shock decreases as the component absorbs (and survives) more shocks, its life distribution is NBU. [See Hollander and Proschan (1972) for the NBU derivation.] For example, if the component's survival probability can be written as

$$
S(t)=e^{-\left[\frac{t}{h}\right]}, t \geq 0
$$

where $[x]$ denotes the largest integer $\leq x$ and $h$ denotes the length of time between shocks (made constant here for simplicity), the resulting lifetime distribution is NBU but not IFRA.

To test whether an underlying lifetime distribution is NBU, define $T_{n}$ as the number of subgroups of size 3 (out of $n$ ) in the data for which one of the items in the group of three is bigger than the sum of the remaining two. Note that $T_{n}$ is an integer between 0 and $n(n-1)(n-2) / 6$ and is proportional to the U-statistic used in rank-test from nonparametric statistical theory [for example see, Lehmann (1975)]. The null-hypothesis that the distribution has constant failure rate is rejected in favor of the hypothesis that the distribution is NBU if $T_{n}$ is sufficiently small. Table values for $T_{n}$, based on $n \leq 20$, are found in Hollander and Proschan (1972). For larger $n$, approximate tests can be constructed by treating

$$
\left(\frac{2 T_{n}}{n(n-1)(n-2)}-\frac{1}{4}\right) \sqrt{\frac{432 n}{5}}
$$


as a standard normal random variable. An NBU test will detect deterioration in component groups that show initial wear but also can include groups for which aging is negligible beyond the initial wear-in period. Components with high reliability and long lifetimes are typically NBU but do not necessarily have an increasing failure rate (average). For such components, we may not consider the overall effects of aging significant.

Of the three tests, the IFRA test is sensitive to the overall effects of component aging (unlike the IFR test) without falsely detecting aging effects where only an initial wear-in effect exists (as does the NBU test). To illustrate the test procedures, we applied all three nonparametric procedures to some component data from Wolford et al. (1992) and entered the results in Table 3-3. A p-value in Table 3-3 is defined as probability of obtaining data that is more contrary to the hypothesis of constant failure rate (as opposed to a failure distribution from an alternative aging class) when, in fact, the true failure rate is constant. However, the failure times we used for the various pumps and valves were interpolated from graphs and may be slightly off from the true data. Again, a perfect-repair model is assumed, which implies that the recorded failures represent the entire lot of available data. The NBU test liberally detects aging in some components for which the IFR and IFRA test do not, as we expected. The results of the nonparametric tests are not in complete agreement with the related parametric tests from Wolford, et al. (1992). The parametric tests are constructed to detect the significance of the unknown shape parameter under the stipulation of the PFRM that is given. Whether or not the parameter is significant (and hence the failure rate is significantly increasing in time) may be made unclear if the PFRM is not suited to the data.

Tables for the IFR/IFRA tests in Klefsjo are limited to values of $n=5,10,15, \ldots, 75$. Interpolated values can be used for other values of $n$, and the standardized test statistic for each procedure is expected to perform well if $n$ is large, but it is not clear how large the sample must be to retain accuracy for a hypothesis test with a fixed type I error. Type I error is defined as the probability of rejecting the null hypothesis (that the components have a constant failure rate) in favor of the proposed failure rate (IFR, IFRA, or NBU) when the null hypothesis is actually true. For fixed error rates of 0.01 and 0.05 , Figs. 3.10 and 3.11 show that both the IFR and IFRA standardized test statistics do not necessarily lead to accurate test results if the sample size is small. For the IFRA test, $B^{*}$ should be used only if $n>10$. For the IFR test, we should obtain a sample of 25 or more before we use $A^{*}$ as a standard normal random variable. For samples smaller than 20, the type I error perceived by the IFR test is much smaller than the actually type I error obtained; thus, the test procedure can lead to confidence statements that are inflated beyond the truth. For larger set error rates (say, 0.10 or 0.20 ), the sample size requirement is not as large.

\subsubsection{Alternative Methods of Failure Rate Estimation}

When it is determined that an aging effect exists in the lifetime distribution of a component group, we may want to use the data to describe the aging effect as a function of time, like the PFRMs. One intuitive alternative to the parametric models already suggested is a Bayesian scheme. Because data are often sparse and because expert opinion and subjective prior information about component lifetimes and aging mechanisms are prevalent in the research, Bayes estimators for component lifetimes and component failure rates may be well suited for the analysis. A method using Bayes' rule presented in Bier et al. (1991) discretizes the time interval, which simplifies the estimation problem without over-parameterizing the failure rate function. Bier's approach and its use of plant-specific data are discussed in detail in Sec. 3.2.1.

A more standard Bayesian method models the lifetime using a known parametric family (e.g., a Weibull distribution) so that the parameterized failure rate is flexible. Unfortunately, for prior distributions like the Weibull, no conjugate family exists for the corresponding data, so analysis involving the posterior distribution is computationally difficult. [See Martz and Waller (1982) for a discussion of the different selections of prior distributions.] 
Table 3-3 Results of nonparametric tests

\begin{tabular}{|c|c|c|c|c|}
\hline $\begin{array}{l}\text { Component } \\
\text { Group }\end{array}$ & $\underline{N}$ & $\begin{array}{l}\text { IFR Test } \\
\text { P-value }\end{array}$ & $\begin{array}{l}\text { IFRA Test } \\
\text { P-value }\end{array}$ & $\begin{array}{l}\text { NBU Test } \\
\text { P-value }\end{array}$ \\
\hline $\begin{array}{l}\text { Motor } \\
\text { Operated } \\
\text { Valves } \\
\text { (Plugged) }\end{array}$ & 42 & 0.5113 & 0.0001 & 0.0001 \\
\hline $\begin{array}{l}\text { Motor } \\
\text { Operated } \\
\text { Valves (FSC) }\end{array}$ & 12 & 0.0277 & 0.0157 & 0.003 \\
\hline $\begin{array}{l}\text { Pumps } \\
\text { (Failure to } \\
\text { Run) }\end{array}$ & & & & \\
\hline $\begin{array}{l}\text { 1-TDP } \\
\text { 2-TDP } \\
\text { TDP }\end{array}$ & $\begin{array}{l}13 \\
11 \\
24\end{array}$ & $\begin{array}{l}0.3657 \\
0.0001 \\
0.03\end{array}$ & $\begin{array}{l}0.5385 \\
0.0001 \\
0.0009\end{array}$ & $\begin{array}{l}0.2471 \\
0.0001 \\
0.0001\end{array}$ \\
\hline $\begin{array}{l}\text { (Combined) } \\
\text { MPD } \\
\text { (Combined) }\end{array}$ & 11 & 0.035 & 0.001 & 0.0019 \\
\hline $\begin{array}{l}\text { Pumps } \\
\text { (Failure to } \\
\text { Start) }\end{array}$ & & & & \\
\hline $\begin{array}{l}\text { 1-MDP-A } \\
\text { 1-MDP-B } \\
\text { 2-MDP-A } \\
\text { 2-MDP-B } \\
\text { MDP }\end{array}$ & $\begin{array}{l}5 \\
3 \\
4 \\
4 \\
16\end{array}$ & $\begin{array}{l}0.0002 \\
0.0002 \\
0.1237 \\
0.2781 \\
0.032\end{array}$ & $\begin{array}{l}0.0009 \\
0.0886 \\
0.2785 \\
0.227 \\
0.0041\end{array}$ & $\begin{array}{l}0.0001 \\
0.0001 \\
0.0607 \\
0.5 \\
0.0001\end{array}$ \\
\hline
\end{tabular}




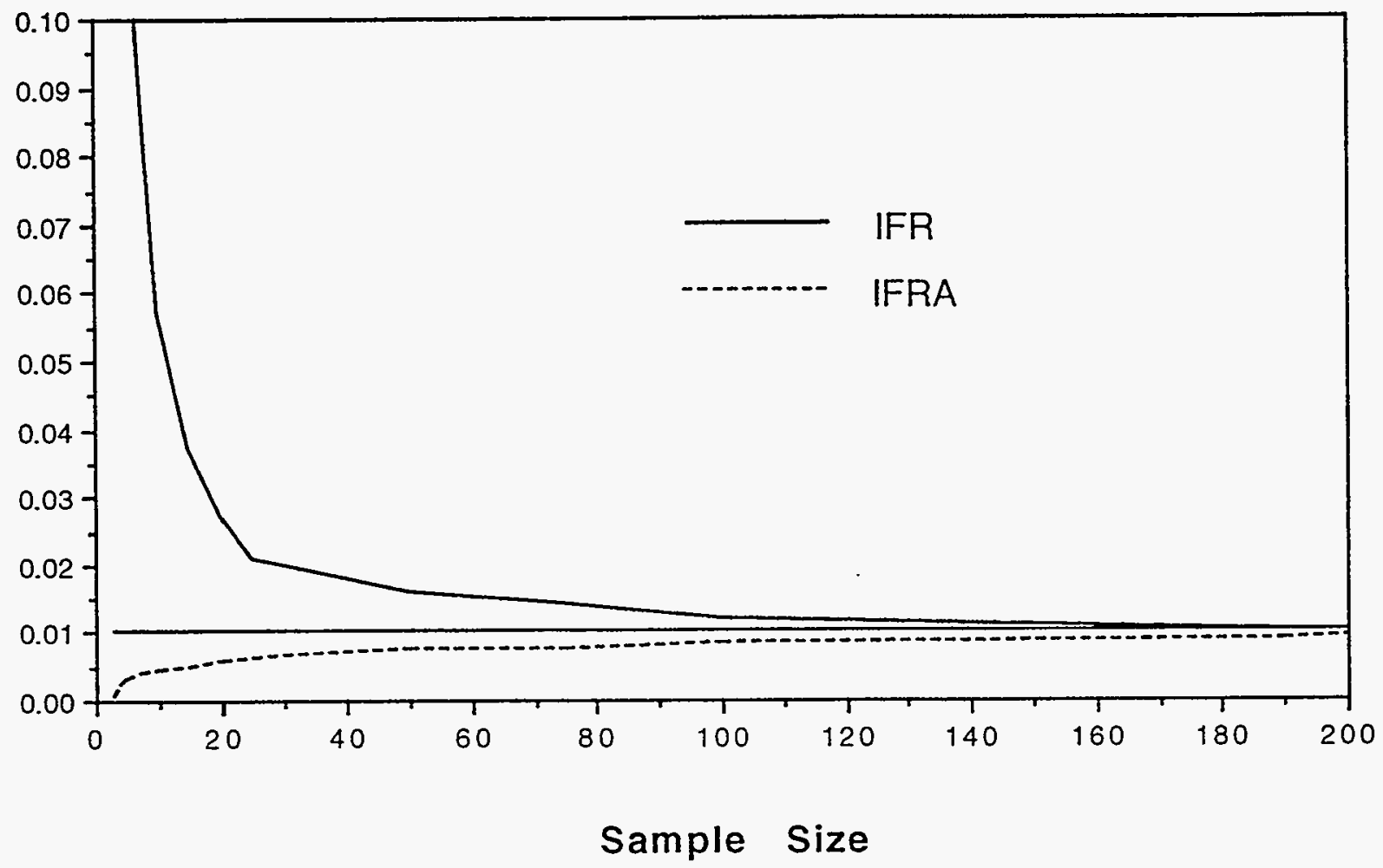

Figure 3.10 Actual Type I error $($ alpha $=0.01)$ 


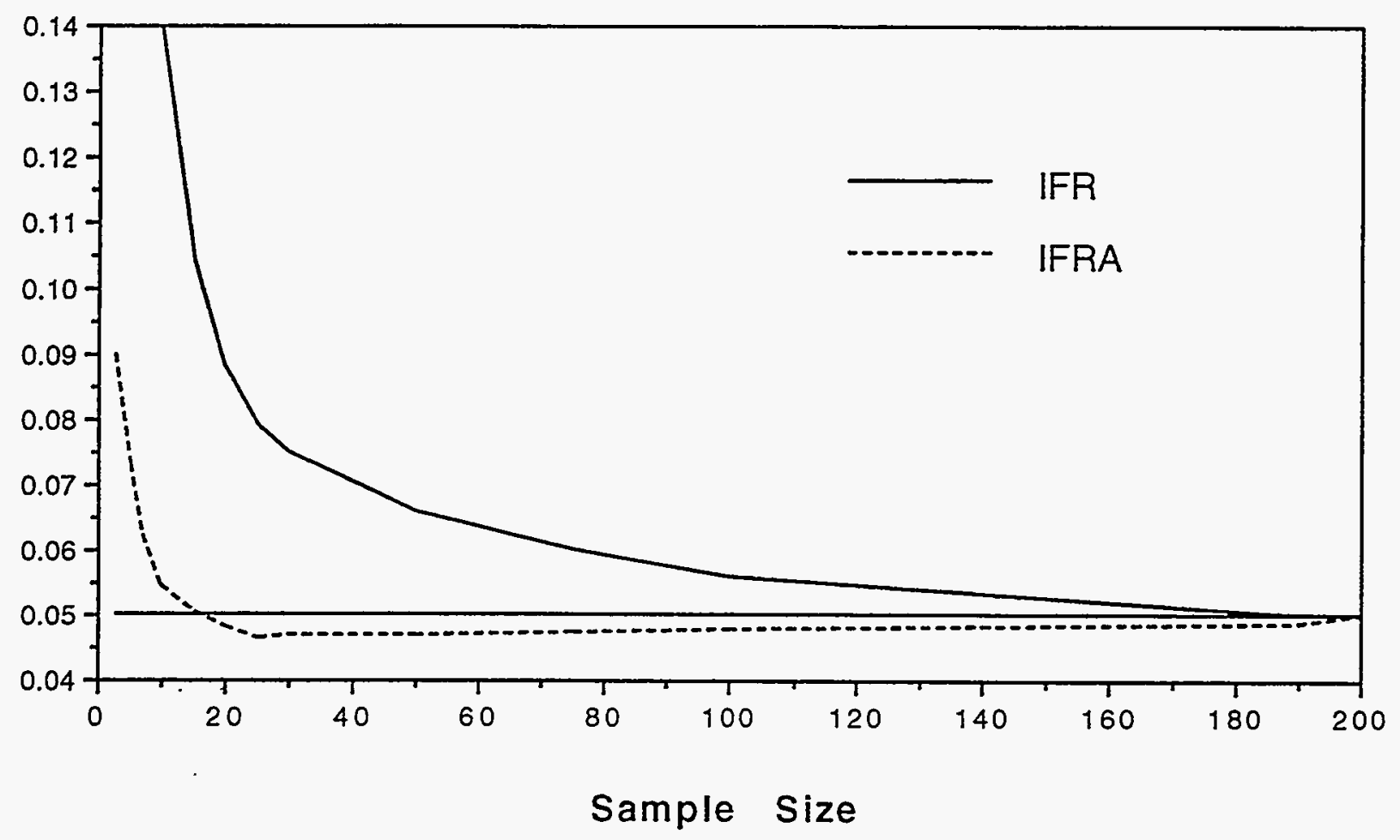

Figure 3.11 Actual Type I error (alpha $=0.05$ ) 
Non-Bayesian, nonparametric options for further inference of the failure rate include the Kaplan-Meier estimator [Kaplan and Meier (1958)] or KME and the life-table estimator (LTE) used in survival analysis. Both estimators are described in Miller (1981) and can easily be employed using a SAS program. The KME is a product-limit estimator for the survival function and works effectively even if time-censored (success) data exist. However, in comparison to the parametric methods presented in Wolford et al. (1992) these nonparametric estimators are limited in scope. In most cases, extrapolation beyond the data is met with great uncertainty, making such estimates seem inferior.

Another nonparametric approach uses Kalman filtering techniques to forecast future failure rate and reliability based on failure data obtained by looking back a certain amount of time from the point of estimation. For this estimation approach, we think of the time-ordered component failures as a stochastic process generated as a time series. The analysis is inevitably more complex than the others mentioned but is also more flexible than the nonparametric procedures and relies on fewer assumptions than the parametric procedures. By design, the Kalman filtering allows the user to emphasize forecasting more than can be done with the previously mentioned estimators.

The technique of Kalman filtering will not be explained here. Estimation of failure rates by means of Kalman filtering is explained in Martz et al. (1977) and also described as a tool in reliability in Breipohl (1969). Although this estimation method is promising in terms of flexibility and forecasting, it is unproven in practice. Also, trends in the failure rate must be modeled by first plotting the data in terms of component reliability or failure rate, so the modeling is partially subjective.

\subsubsection{Discussion}

Several alternatives to the PFRMs in (Wolford et al., 1992) have been reviewed, including nonparametric tests of failure rate (IFR, IFRA, and NBU), nonparametric estimators of reliability (KME and LTE), nonparametric estimator of the failure rate process (Kalman filtering), and Bayes' estimation of the failure rate. Mann, Schafer, and Singpurwalla (1977) contains lengthy discussion of these and other related parametric estimation techniques. Barlow and Proschan (1981) focus on nonparametric estimation of reliability and failure rate.

It is recommended that failure data are first tested for increasing failure rate average because lifetime deterioration as a result of aging seems to correspond to the IFRA family of distributions. If no IFRA seems to be present in the component data, intricate parametric modeling of the failure rate as a function of time may not be appropriate for the study.

If the failure rate seems to be increasing over time, we should proceed with statistical inference to answer additional questions about the effects of aging on component lifetimes. If sample sizes are large enough, the parametric models discussed in NUREG/CR-5378 should be explored. Model assessment is described thoroughly in that report, including suggested tests for model lack of fit, tests for pooling common lifetime data, and tests of parameter significance. The inference procedure is laid out in several basic steps and will lead the user to accurate and descriptive statistical conclusions provided the demands of the model are met. If the failure times appear clustered or otherwise do not fit the PFRMs adequately, alternative methods of estimation are prescribed.

The greatest problem with the parametric models of component lifetime is the lack of data available to test such models. In Wolford et al. (1992) and Atwood (1992), it was found that the exponential and Weibull failure rate models work better than the linear failure rate models. In the exponential and Weibull models, estimates and confidence bounds for $\beta$ are always finite, and the asymptotic normal distribution is approached even with samples of only moderate size. 


\subsection{Conclusions}

The TIRGALEX data base was discussed in Sec. 3.1 and is the result of an expert panel workshop held in May 1987. It provides the input necessary to conduct the RSCA, which is an evaluation of a component's contribution to plant risk because of aging, and RSCAAMP, which is an evaluation of the effectiveness of current industry-management practices for maintaining an acceptable risk level of plant in the presence of aging components. The objective of the TIRGALEX program is to set the priorities of the aging evaluation of the structures and components. The TIRGALEX data base also was used in later probabilistic risk studies (Vesely et al., 1990; Vesely, 1992) under the NPAR program.

The following conclusions were drawn regarding the TIRGALEX data base.

(1) The data base is the result of unstructured expert opinion elicitation procedures, and as such, it is subject to well-known biases, such as location and variability biases. (In other words, the values reported may be "widely off the mark.") Uncertainty ranges are not reported.

(2) Many of the estimated quantities are very plant-specific, yet they are assessed generically without any uncertainty statement.

(3) Optimum test intervals, as well as the probability of successful detection of degradation and the probability of successfully mitigating the degradation given detection, depend on the specific aging mechanisms at work, yet no specific aging mechanisms are considered. This comment is related to our finding in the Sec. 2 report that the use of failure rates masks the physics behind the various contributors to risk, thus inhibiting risk management.

The use of generic and plant-specific operating experience data for identifying aging mechanisms in active components was reviewed in Sec. 3.2. In general, although a large amount of information regarding the reliability of active components is available, there are several problems with using this data for assessing the aging of active components. Particular problems are listed below.

(1) No single data source, generic or plant-specific, provides the necessary detail to perform a detailed analysis of component aging analysis.

(2) Although a combination of various sources may allow a qualitative evaluation of the failure modes, causes, and aging mechanisms, it is difficult to reach any quantitative conclusions.

(3) In many cases, additional difficulties in the data analyses have been introduced because some vendors, utilities, and manufacturers have been reluctant to provide the necessary information.

(4) Data from operating experience are not sufficient in quantity or quality for validating any of the proposed aging models (linear, exponential, or the Weibull aging model), and the uncertainty level in the estimate of failure rates and especially in the rate of change of failure rates remains high.

In Sec. 3.3, existing physical models for aging mechanisms of passive components and the data available pertaining to these models were discussed. In general, the data required for the physically based reliability models of passive components consists of material properties and component stressors. The availability and quality of such data varies and depends on the particular component and mechanism being studied. 
In Sec. 3.4, statistical methods for describing and evaluating the effects of aging on component lifetimes were discussed. Parametric failure rate models were described, and alternative methods for the statistical evaluation of data related to aging were presented and discussed. The greatest problem with the parametric models of component lifetime is the lack of data available to test such models.

Finally, it should be pointed out that with respect to aging mechanisms, there is a wealth of information available in the form of operating and maintenance experience. This information is particularly important for passive components, where few actual failures have been observed but degradation related to aging has. However, the immediate usefulness of this information in terms of quantitative measures of reliability and risk related to aging is limited because this information is qualitative in nature. However, it is valuable for providing insights about which aging mechanisms are affecting which SSCs and the relative importance of those mechanisms. 


\section{INTEGRATED AGING RISK ANALYSIS}

\subsection{Introduction}

A review of PRA methodology in connection with NPP aging reveals the following.

(1) The current PRA methodology does not adequately address the aging of SSCs at NPPs. In particular, one assumption made in the current PRA models is that component failure rates are constant over time. Another area of concern is that, under the current PRA practice, component failure modes that traditionally had been judged as negligible for new plants might become important risk contributors for aging plants. Most significant in this regard is the possible increase in the failure probabilities of major passive components (e.g., the $\mathrm{RPV}$, the reactor coolant piping, etc.).

(2) A large amount of information, both quantitative and qualitative, has been gathered from the NPAR program that has added to the knowledge base on the issue of NPP aging. However, this body of knowledge has not yet been integrated successfully into formalisms that can be used effectively and systematically in aging-related risk assessments. In NPAR reports, various components and aging mechanisms that are critical to plant safety are identified and inspection and maintenance strategies often are suggested. However, these reports are limited thus far to individual components and specific mechanisms. An integrated risk study that amalgamates various bodies of information would be useful in providing insights regarding the safety of an aging plant.

(3) Several risk models have been proposed to address the issue of equipment aging in risk assessments, e.g., the Taylor series expansion (Vesely et al., 1990; Vesely 1992) and the maintenance effectiveness evaluations in NUREG/CR-5612 (Samanta et al., 1991). A main drawback of these models is that they rely on assumptions regarding the behavior of timedependent failure rates without any consideration of physical mechanisms. In doing so, important physical phenomena and their causes remain obscure when component failure rates are estimated. One result of these approaches is that risk assessment is kept apart from physical mechanisms and conditions that may lead to failure. This will largely mask the physics behind various contributors to risk, thus inhibiting the development of effective risk-management strategies.

Based on these observations, we have concluded that to address the risk at an aging plant adequately, an extension of current PRA methodology is necessary and essential to the understanding of the overall risk involved at a nuclear power plant. However, extension of the current methodology would be more expedient if the effect of aging on plant risk is assessed within a framework that is built on existing PRA models.

In the model that we propose in this section, the effect of aging on plant risk will be analyzed in a separate section of the PRA and based largely on the results of "internal" event analyses. This method of treating aging events "externally" is similar to that for other hazards such as seismic events, fires, and flooding.

In this section, we will discuss the framework of such an additional section in the PRA. The findings in Secs. 1-3 of this report will be integrated here to identify areas where the current PRA needs revision, to develop methods that can be used to model aging risk, and to investigate the issue of data adequacy for aging-risk analysis. 


\subsection{Proposed Aging-Risk Analysis}

Figure 4.1 shows the scope of our proposed aging-risk analysis, which covers the fundamental issues mentioned above. The analysis comprises three major parts: the selection of aging components and mechanisms, the physical models of selected components and mechanisms, and the integration of the findings from the first two into the PRA.

The analysis starts with an effort to select a set of aging SSCs, as well as mechanisms that are significant to plant safety because it is necessary to limit the scope of the analysis to a manageable size. Physical models, such as probabilistic structural mechanics, involve complicated formalisms and require detailed information. Such an approach would be ineffective when a large number of components must be evaluated. Therefore, it is essential to construct a set of screening criteria. This would allow analysts to identify the components and their associated aging mechanisms that are significant to plant risk and need further investigation. Section 4.3 will discuss several approaches that can be used in the screening analysis. However, more work is needed before a comprehensive set of screening criteria can be proposed.

The first part of the analysis will result in the identification of a set of SSCs and mechanisms to be investigated further. The second part of the analysis will involve the development of physical models based on these failure probabilities of the selected components and mechanisms that can be estimated. Several physical models are suitable for our purposes and can be used directly. One example is the software package, PRAISE, which was developed to evaluate aging risk resulting from component fatigue. For other aging mechanisms such as erosion/corrosion, more work is needed to develop better physical models.

As shown in Fig. 4.1, the inspection, test, and maintenance practices at specific plants directly effect the time-dependent component failure rates. These practices should be modeled explicitly in the second part of the analysis.

The third part of the proposed aging-risk analysis is to integrate the effect of plant aging into the existing PRAs. Initiating events, event trees, and fault trees in PRAs will be reviewed and updated based on the findings from the screening analysis and physical models. The modification of PRAs may include adding of basic events to fault trees or constructing new event trees and fault trees. Core-damage frequency and public risk will be quantified using the estimated failure rates of selected components that will be obtained during an earlier phase of the analysis.

This proposed approach retains most of the basic structure of current PRA models. In developing an extension that includes plant aging risk, we have examined various models that have been introduced in earlier research and found that the model we are proposing has several major advantages that we discuss below.

One reference model that we examined is one that has been described in Phillips et al. (1992) in which the risk significance of passive components was investigated using PRA techniques. In addressing the issue of passive component aging, the study proposed using simple screening methods in setting up analysis priorities, and probabilistic structural analysis was recommended for estimating component failure rates. When the significant passive components are identified and the aging failure rates are estimated, the PRA would be modified to incorporate the contribution that passive components make to risk.

The framework that we are proposing is similar to the approach by Phillips but with a broader view. Instead of singling out passive components, our model is designed to address both "active" and "passive" components and to include physical models on all major aging mechanisms. Plant operating experience has shown that some aging-related failure modes of "active" components at NPPs have 


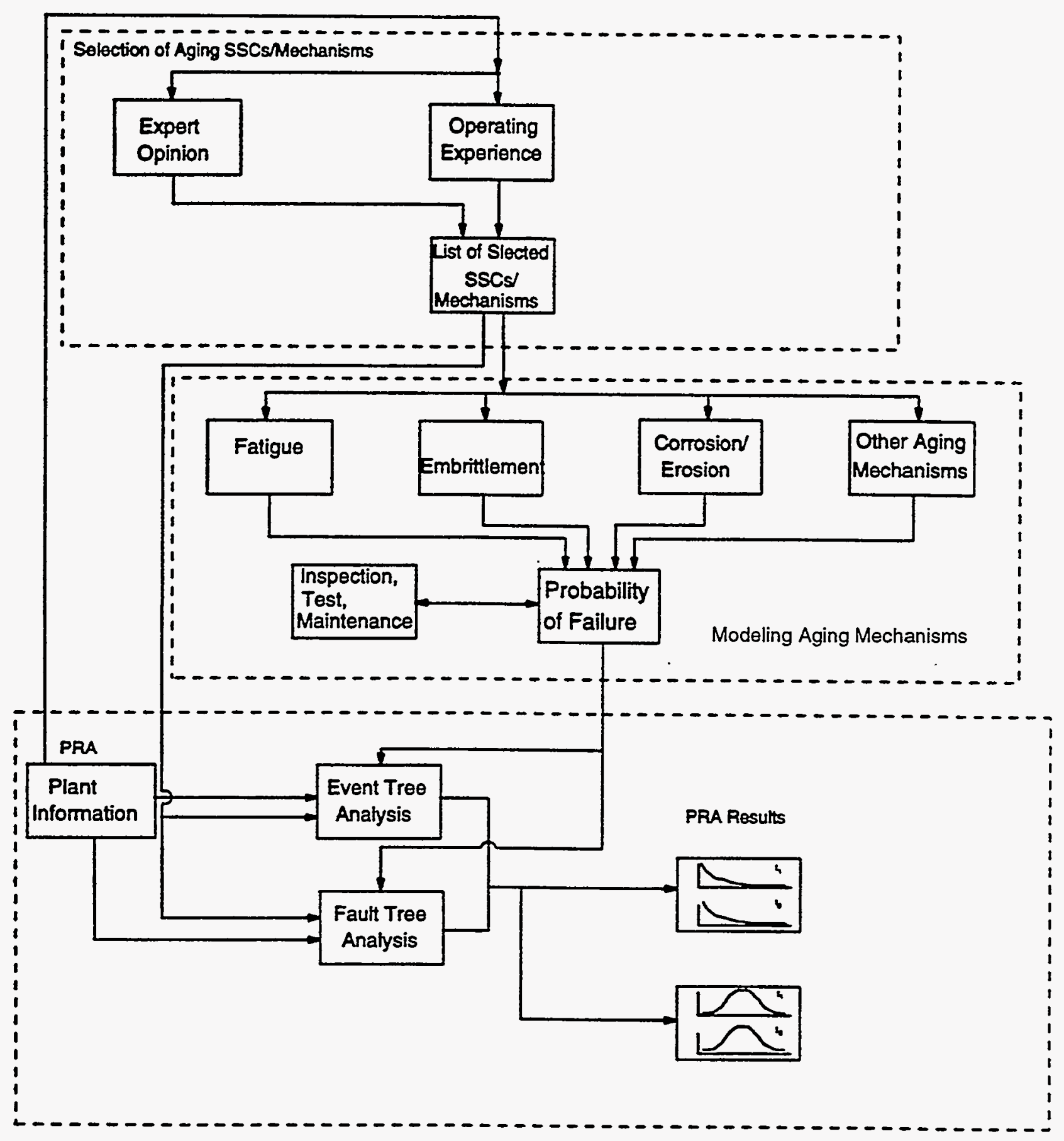

Figure 4.1 Aging-risk analysis 
similar characteristics to their passive counterparts in that they are relatively negligible during the early years of plant operation but would become significant toward the end of life. These late-failure possibilities of active components cannot be identified easily under current inspection practices. Additionally, statistical data are not sufficient at the present time to support any empirical correlations that have been proposed for modeling the change of component failure rate over time. An example of late failures of active component is the valve-stem cracking that has been observed at aging plants. Without effective inspection practices, cracks will be hard to identify in the early stages of propagation.

In fact, the distinction between passive and active components at NPPs in terms of component aging is not as clear as it is in terms of operation. In aging-risk analysis, the main concern is not whether a component is active or passive, but rather how significant the risk to the plant would be if a component failed.

The current approach is designed to analyze various degradation mechanisms. Components in on-line systems may age from excessive use, whereas stand-by components may age from excessive testing and environmental conditions. Plant-specific inspection, testing, and maintenance practices also may significantly affect equipment aging. Because the mechanisms involved in various aging processes differ in their physical nature, failure probabilities should be assessed separately for each different cause of age-related failure.

Our literature review in Sec. 2 shows that our understanding of the physical phenomena involved in aging mechanisms is relatively poor. This is especially true in the study of the probabilistic nature of these phenomena. For example, although computer software such as PRAISE has been developed for estimating the fatigue reliability of piping and welding, models developed for irradiation embrittlement or corrosion/erosion are largely deterministic. Because the current approach emphasizes the integration of physical mechanisms and probabilistic models, more research is needed in areas where the two types of approaches have not yet reached a balance.

\subsection{Selection of Aging Components and Aging Mechanisms}

As pointed out by Phillips et al. (1992), probabilistic structural analyses and fracture mechanical analyses are difficult and costly to perform; therefore, evaluating every single component in a plant would be extremely expensive. Identifying all input parameters (stresses, material properties, etc.) for a given component is often difficult and subject to variation. In addition to the analytical complexity, data gathering also involves a major effort, particularly when projecting the effects of aging in the future. Simplified methods that screen out the less risk-significant components and prioritize the most risk-significant ones would be very useful in this context. The time-consuming probabilistic structural-analysis techniques or fracture mechanical analysis then could be used for those selected components for which detailed evaluation is necessary.

The selection of aging components and mechanisms for further investigation will be based on two general categories of criteria, risk-based and reasonable-assurance. The risk-based criteria are to ensure that resources are spent on areas of risk significance, and the reasonable-assurance criteria are to ensure that items of large uncertainty get proper attention. Depending on the nature of the aging mechanisms and the current knowledge of component failures, different approaches may be required for different mechanisms in the screening process. In this section, we discuss three tools (engineering judgment, operating experience, and risk-significance analysis) that can be used in the screening process and the limitations of each tool to be overcome. 


\subsubsection{Engineering Judgment}

Engineering judgment based on extensive field experience is one of the tools often used to find solutions for complex problems. Engineering judgment will be used as a vital resource in the screening process of the aging-risk analysis, especially for identifying components for which the current knowledge involves large uncertainty. For components so identified, more detailed analyses will be needed to be reasonably sure of their reliability. Failure modes of components of a "passive" nature are more likely to fall into this group because of the lack of data.

Engineering judgment has been used frequently in NPAR studies. For example, it has been suggested by Shah et al. (1987) that the primary degradation mechanism affecting primary-coolant piping in PWRs is low-cycle fatigue. This was judged to have been caused by a combination of pressure and transient thermal stresses that concentrate near nozzle welds and near the terminal ends of the piping. In another section of the report, it was stated that fatigue is the primary degradation mechanism affecting BWR reactor pressure vessels. The report continued by stating that three of the highest ranking degradation sites in older plants are the austenitic stainless-steel safe ends that have been sensitized by post-weld heat treatment, the crevice areas where thermal sleeves are attached to Iconel or stainless-steel safe ends, and the austenitic cladding on the inside corners and surrounding regions of the nozzles. Findings such as these are based on extensive engineering experience and need to be collected systematically for further analysis.

Table 2-1 summarizes the safety-related components and their associated degradation mechanisms that have been studied in the NPAR program and are possibly worthy of further investigation. The list is based largely on operating experience and engineering judgment.

\subsubsection{Operating Experience}

A second tool that can be used for screening is the analysis of operational experience. If data are sufficient for making statistical inference, statistical data analyses also can be used to determine time-dependent failure-probability parameters in risk evaluation. Unfortunately, related studies under the NPAR program have shown that existing data sources are not sufficient for statistically validating the existing aging models. The uncertainty level remains high in the estimate of failure rates and in the rate-of-change of failure rates.

\subsubsection{Risk-Significance Analysis}

Risk-significance analysis also can be used in the screening process. This approach uses information gained from existing risk analyses to estimate the risk significance of plant components. Risk significance will be considered in terms of both frequency and consequences of component failures. Because only the components that are considered risk-significant need further evaluation, limited sensitivity analysis can be conducted to estimate the ranges of risk significance. This technique will be used to screen out a significant number of components for which the consequences are too low to be worthy of further analysis.

The TIRGALEX exercise (Levy et al., 1988) under the NPAR program is one form of significance analysis. The goal of the TIRGALEX exercise is to identify safety-related structures and components that should receive high priority in subsequent phases of the NPAR program. The TIRGALEX panel combined information from existing PRA with experimental information and derived a list of components that need further evaluation. When practicing TIRGALEX-like screening analysis, analysts should note several points. 
The TIRGALEX data base is a result of unstructured procedures to elicit expert opinion and, as such, is subject to biases. In addition, although the values reported are subject to large uncertainties, the issue of uncertainty has never been addressed formally during the TIRGALEX exercise.

Furthermore, test intervals and inspection and repair probabilities depend on specific aging mechanisms. Although one inspection technique may be effective in finding a specific type of failure mechanism, it may have little probability in finding another type of aging-related mechanism. The TIRGALEX panel did not address this problem.

\subsection{Probabilistic Physical Models of Aging Mechanisms}

\subsubsection{Fatigue}

Extensive probabilistic models and computer codes have been developed for fatigue in pipes and vessels. One methodology is the software, PRAISE, which was developed to estimate the reliability of aging pipes at nuclear power plants (Fullwood and Hall, 1990). The code models crack growth in a piping section and uses Monte-Carlo simulation to compute the failure probability of the pipe at a given time, $t$. Similar models have been applied to estimate the failure probabilities of nuclear vessels (Becher and Pedersen, 1974) and piping (Harris and Lim, 1982).

Figure 2.1 shows a general flow chart of the PRAISE model. The starting point for the calculation is the distribution of microflaws in the pipe base metal that are introduced as a result of fabrication (box A). Residual and transient stresses on the pipe cause these cracks to grow, but there is a probability that they will be detected and repaired before they reach catastrophic levels. The nondetection probability of a crack is represented by box $\mathrm{B}$. The combination of the initial distribution of cracks and the nondetection probability distribution results in a post-inspection distribution. Box $E$ is where the crack size as a function of time is calculated, according to a stress history. Box $D$ represents the model that relates the crack-growth rate to the applied stress and the calculated stress-intensityfactor, which is represented by box $C$. The other boxes are related to failure modes and criteria and to the leak-before-break issue.

For research pertaining to the initial crack-size distribution, Marshall (1982) presents data from cracks found in nuclear and nonnuclear pressure vessels in the US and in the UK and concludes that the crack sizes are distributed exponentially where the aspect ratio is constant. The PRAISE model uses a truncated exponential but a distributed aspect ratio for the crack size.

For the nondetection probability of a given crack size, the PRAISE model uses a complementary error function based on data given in (Harris, 1977). Yang (1976) uses a simple truncated exponential function for the probability of detecting a crack as a function of crack size in the model he developed for estimating the distribution of crack sizes in aircraft. Yang's model accounts for the statistical distribution of the initial crack size, the reliability of the nondestructive inspection (NDI) technique used for detecting the cracks and the crack propagation of repaired and unrepaired elements. In the model developed for the probability of fatigue-damage accumulation, Sampath and Broek (1991) also use a truncated exponential function; the data were based on Lewis et al. (1978).

To model crack propagation, many crack-propagation laws have been proposed to estimate the crack-growth rates. For instance, the Paris-Erdogan equation discussed in Sec. 2 has been used in the PRAISE model to describe the characteristics of crack growth.

\subsubsection{Thermal Embrittlement}

In the thermal environment of a PWR, ferritic and martensitic steels become embrittled by the precipitation of a b.c.c. chromium-rich phase in the temperature range of PWR operation $\left(290^{\circ} \mathrm{C}\right.$ - 
$350^{\circ} \mathrm{C}$ ). Precipitation of the chromium-rich $\alpha^{\prime}$ phase is associated with hardening, as measured by the increase in the Vicker's hardness number (VHN) (Meyzaud et al., 1984). The increase in the VHN (i.e., $\triangle V H N$ ) has been correlated to the increase in the volume fraction of the $\alpha^{\prime}$ phase up to a maximum value that is strongly dependent on the age (or time). For example, the maximum $\triangle V H N$ is attained within about $1 \mathrm{yr}$ at $450^{\circ} \mathrm{C}$. However, this increase is much smaller at operational temperatures.

It has been found that several alloying elements influence the degree of thermal embrittlement; the most prominent are $\mathrm{Cr}, \mathrm{Mo}, \mathrm{Al}$, and $\mathrm{Cu}$. Empirical studies suggest that a parameter, $\mathrm{Cr}^{*}$, be used to correlate with the maximum amount of thermal embrittlement; i.e.,

$$
\mathrm{C}_{\mathrm{r}}^{*}=\% \mathrm{Cr}+\% \mathrm{Mo}-\mathrm{A}(\% \mathrm{C}),
$$

where $A$ is a constant in the range of 12 to 16 , depending on the type of carbides formed. The maximum hardening also depends on the quantity of $\gamma$-phase austenite; i.e.,

$$
(\Delta \mathrm{VHN})_{\max }=[22.1-0.313(\% \gamma)]\left(\mathrm{Cr}^{*}-10.2\right) \text {. }
$$

It is also empirically established that both the upper shelf energy and the shift in the CVN transition temperature can be correlated to the VHN shift (Meyzaud and Cozar, 1984; Courtnall and Pickerin, 1976). The shift in the CVN transition temperature as a result of aging is approximately proportional to the corresponding increase in the hardness; i.e.,

$$
\Delta \mathrm{T}\left({ }^{\circ} \mathrm{C}\right)=-12+2.17(\Delta \mathrm{VHN}) \text {. }
$$

At temperatures between $300^{\circ} \mathrm{C}$ and $450^{\circ} \mathrm{C}$, aging was found to cause a decrease in the USE and an increase in the DBTT. It is proposed here that correlations be found between the $\triangle D B T T$ because of aging and the critical stress-intensity factor for fracture, $K_{C}$, under the three modes of failure. Should this correlation be found, then $\mathrm{K}_{C}$ will be a decreasing function of time. If material uncertainties are included, a probability distribution function, pdf, for $\mathrm{K}_{\mathrm{C}}$ can be derived. Thus, the pdf would be expected to shift with age, leading to lower levels of toughness for crack propagation.

The stress distribution at screened critical locations in the piping system also should be determined, including uncertainties in component loads and geometry. We would construct a stressstrength interference model similar to what has been proposed for radiation embrittlement of pressure vessels where the failure rate can be computed as a function of time.

\subsubsection{Erosion/Corrosion}

Erosion/corrosion of NPP pipings has caused several pipe ruptures (INPO, 1987; Virginia Power Company, 1987; USNRC, 1987). Such failures have led to substantial efforts by the nuclear industry to mitigate the problem (EPRI, 1987a and 1987b). Erosion/corrosion mechanisms acting on specific locations can lead to unacceptable thinning of pipe walls, and monitoring, maintenance, and repair are required to prevent pipe failure in these situations.

Nondestructive examination (NDE) of carbon-steel piping for erosion/corrosion wall thinning has been documented in an EPRI report (EPRI 1987c), where guidance for conducting these tests also was given. In the EPRI report, measurements are compared with component nominal wall thickness, $t_{n o m}$. The current guidelines are based on a deterministic logic process for acceptance, repair, or replacement of the piping component, as shown in Fig. 4.2, taken from another EPRI report (1988). Wall thickness variations from nominal up to $12.5 \%$ are allowed. If the measured thickness is within this tolerance, no further evaluation is required. On the other hand, if the measured wall thickness is $<20 \%$ of the nominal design value, component repair or replacement is implemented. 


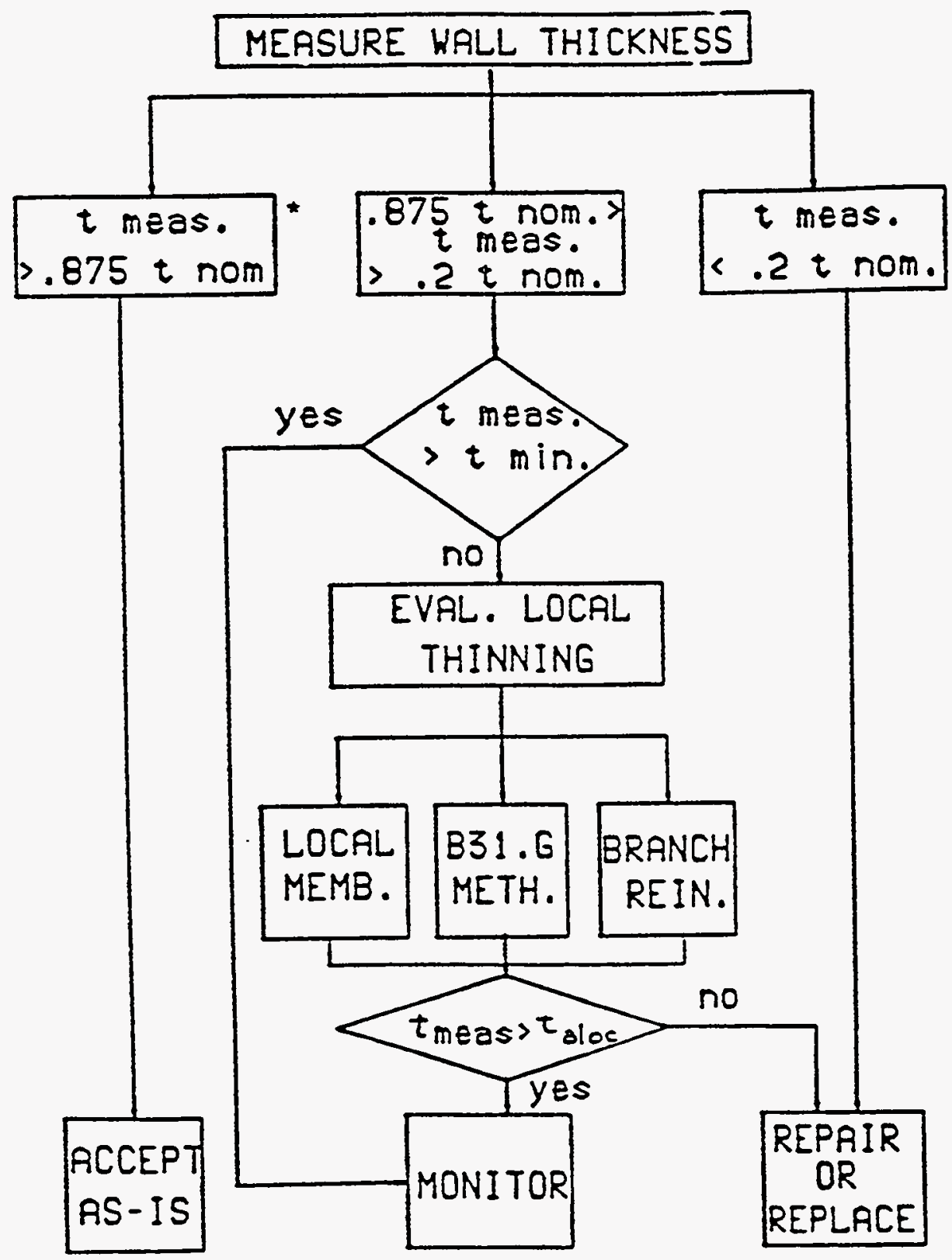

Figure 4.2 Evaluation of wall-thinning logic diagram (EPRI, 1988) 
The number of fundamental studies of synergistic erosion/corrosion is rather limited (Natesan and Liu, 1989). Wright et al. (1986) identified the principal feature that may be observed in actual experiments as the intensity of erosion becomes increasingly severe. They considered two possible scenarios: (1) the kinetic energy of the eroding particles is relatively low and (2) the kinetic energy is high (as in the case where particles have sufficient energy to penetrate the oxide film, the removal displays the characteristics of brittle erosion). Material removal occurs via chipping or elastic-plastic indentation and fracture. Maximum removal rates occur at near-normal impact angles and with a velocity exponent of 3.5. In response to this localized exfoliation of the surface scale, the oxide layer undergoes accelerated growth in the vicinity of this region.

In the first scenario, because the oxidation and erosion are balanced, the net measured weight change shows a linear rate of loss as the oxide is being removed continuously and the material is being oxidized continuously.

In the second scenario, the impacting particle has sufficient kinetic energy to penetrate the oxide and damage the metal substrate. Even though isolated impacts cause only a little removal of the oxide scale, subsequent impacts on the ploughed crater could detach parts of or all of these features together with the associated scale. The erosion characteristics in this case would correspond to ductile erosion mechanisms. Maximum material loss occurs at shallow impact angles, and the exponent of velocity dependence would be about 2 to 2.5 .

The models for these two regimes are based on rate theory and therefore are deterministic (Natesan and Liu, 1989; Wright et al., 1986). It is suggested that a probabilistic approach to the risk of erosion/corrosion on nuclear-power-plant piping systems be implemented. By doing so, the thinning rate of the piping system will be computed at specific critical locations, which will give the mean rates of removal and the probability distribution function of the wall thickness at these locations. A strength-stress model then can be constructed to determine the probability of pipe failure at the given locations. Obviously, because the pipe is thinning continuously, the average values of the hoop and, to a lesser degree, the axial and bending stresses will increase. Therefore, it is conceivable that the frequency of failure will increase as the piping system ages with time.

\subsection{Probabilistic Risk Assessment}

The last part of the aging-risk analysis is to incorporate the aging effects into the PRA and to estimate the overall effect of aging on plant risk. This section discusses some of the issues that need to be addressed in the current PRA models when considering plant aging.

Two major concerns are associated with the inclusion of aging effects into the PRA.

(1) Some components have very low failure rates at the beginning of plant life, which current PRA models do not include into the plant logic model. In case these aging-related failure modes become significant to plant risk toward the end of plant life, the plant logic model needs to be modified to include them.

(2) The time-dependent nature of component failure rates needs to be addressed and modeled. To keep the analysis focused, only selected components and mechanisms will be considered for detailed analysis. For the majority of components, the plant logic model that was developed in the original PRA will be sufficient. 


\subsubsection{Effect on Core-Damage Frequency}

The effects of aging can be incorporated into the PRA in several ways: constructing new event trees, modifying existing event trees or fault trees, and changing component-failure probabilities or initiating-event frequencies.

In an attempt to incorporate aging effects into the PRA, Vesely et al. (1990) also used the Taylor expansion approach. A major assumption of this approach is that the physical phenomena of the aging process and the plant risk model are independent and can be treated separately. In Vesely's model, the aging model was treated at the component level and risk analysis at the system and plant levels. The Taylor expansion approach then relates the change in overall plant risk to the change in individual component unavailabilities because of aging. The risk formula derived from the PRA is used without modification when estimating aging risk at the plant level.

There are concerns over the Taylor expansion approach from both the conceptual and practical viewpoints on aging risk analysis. Conceptually, the Taylor series-expansion approach imposes stringent constraints on the subjects being analyzed, and one of the major requirements is that the risk "formula" remain the same before and after the introduction of the elements related to plant aging. As shown in Fig. 4.1, in reality, this is not always the case. Aging of a plant may enhance the importance of failure modes that have been judged to be negligible in the basic PRA model. The introduction of additional components and failure modes into the risk model will change the basic structure of event and fault trees and, in turn, the resulting risk formula of the plant. Another source of departure from the base-case PRA risk model is the introduction of new inspection, testing, and maintenance practices to the plant operation. These practices not only change the component-reliability models, but they also introduce new terms, including the repair and maintenance outage and human errors committed during test and maintenance.

In Sec. 2 we concluded that the use of the Taylor series expansion in estimating plant-aging risk through the change of failure rates would be too restrictive because there are situations where factors other than component failure may contribute to plant-aging risk. Moreover, it has not been shown to date how the Taylor-series-expansion approach can be used to estimate the effect of aging risk on seismic or fire risks where there is strong correlation among component failures.

In addition to conceptual obstacles, the Taylor expansion approach also encounters several practical difficulties, one of which is in the choice of component reliability models. Although mathematical models (e.g., exponential and Weibull distributions) were mentioned, only the linear aging model has been evaluated extensively. As is shown in Sec. 2 of this report, the findings were not always favorable. Lacking further guidance, analysts are left to decide which mathematical formula to use in the analyses, and often a decision is made without sufficient knowledge of physics that backs up the formula. Furthermore, as was pointed out earlier, by over-emphasizing component unavailabilities, the physics behind the various contributors to risk will very likely divert the investigator's attention and thus inhibit risk management. For example, in fatigue failure involving crack propagation, the probability distribution of cracks of different sizes is a major factor in aging-risk to the plant, which Vesely's approach will not be able to evaluate.

\subsubsection{Discussion}

An immediate concern over such an extensive analysis as we propose is the quality and availability of the data that would be used in the analysis. Viewing the current status of aging data, one should be prepared to face large uncertainty in the results and a significant amount of subjective judgment used in the analysis. The results also should be viewed with great care. Furthermore, 
decision makers who are engaged in the decisions of aging issues should be trained to be familiar with quantities of large uncertainty and to be able to extract useful information from figures associated with wide range. 
, . 


\section{DATA CRITERIA AND DATA DEVELOPMENT}

\subsection{Introduction}

An aging-risk analysis framework was proposed in Sec. 4 that allows aging to be treated outside to the existing PRA model. The proposed framework consists of three basic parts: the selection of aging components and mechanisms, the physical modeling of aging mechanisms, and the integration of physical models into the PRA. The viability of such an aging-risk analysis depends, to a large extent, on the quality of data available to the analysts. In Sec. 5.2, we discuss the availability and adequacy of the data required for the screening analyses. Section 5.3 discusses data needs for physical models of aging mechanisms.

\subsection{Data Needs for Screening Analyses}

As discussed earlier, two criteria apply toward the selection of components and mechanisms for further investigation. Not only must a component be significant in terms of risk, it also must exhibit age-related degradation. Therefore, part of the screening process must consist of the identification of SSCs that are affected significantly by aging phenomena. Components for which there is no evidence of aging-related degradation can be eliminated from further consideration regardless of their risk significance.

Data sources that can be used in the process of selecting aging components and mechanisms include generic and plant-specific operating experience, plant documents, test and research results, expert opinions, and industrial practices. These sources can be grouped loosely into three categories (i.e., the expert judgment, the industrial operating experience, and the results of engineering analyses) as discussed below.

\subsubsection{Expert Judgment}

A TIRGALEX-like expert panel and workshop can be useful in the setting of screening analysis. The current TIRGALEX approach lacks formal procedures in elicitation, debiasing, and aggregation of expert opinions. A formal evaluation of expert judgment should include structured procedures in the following areas.

(1) The identification and selection of experts

(2) The design and conduct of the elicitation

(3) The treatment of biases of experts and biases induced during the elicitation process

(4) The dependency among experts

(5) The algorithms for aggregating expert judgments

(6) The treatment of uncertainty

In addition, the whole process of formal evaluation should be documented carefully. The qualitative reasoning process in support of the expert judgment constitutes an important portion of the elicitation process and also should be documented in detail. Detailed discussions on the elicitation and use of expert judgment can be found in Keeney and Winterfeldt (1991 and 1989), Ortiz, et al. (1990), Wheeler, et al. (1989), and Chibber and Apostolakis (1992 and 1993). 


\subsubsection{Operating Experience}

Operating experience can provide two types of information: actual occurrences of failures caused by aging and evidence or direct observation of component degradation because of aging. Sources of operating experience information include data from the In-Plant Reliability Data System (IPRDS) sponsored by the NRC, the Nuclear Plant Reliability Data System (NPRDS) managed by INPO, the Licensee Event Reports (LERs), plant maintenance records, in-service inspection reports, and Nuclear Plant Experience (NPE). Among these data sources, LER and NRPDS are the two used most frequently for aging analyses. The NPE database is similar to that of LER, whereas the IPRDS database is very limited and incomplete (Subudhi and Lofaro, 1988).

Actual hardware failures can be evaluated to identify failure modes, mechanisms, and causes. Service environments and applications, together with material and design properties, can be evaluated to identify the nature of, and factors contributing to, aging-related failures. The indicators of failures (for example, leaks, fatigue failures, radiation, thermal embrittlement, etc.) can be assessed to identify aging-related failure modes. Information from maintenance experience and in-service inspection experience can be evaluated to identify components for which aging-related degradation has been observed and the dominant mechanisms affecting those components and can provide qualitative information about the severity of the degradation.

The NPRDS is a large computerized database that contains approximately 500,000 component engineering reports and over 65,000 component failure reports. Each component engineering report contains descriptive information such as manufacturer, model number, the systems where it is installed, capacity, rating, etc. In addition, it also may contain information on testing, such as the method and frequency of tests. Failure reports identify failed components and provide the dates and times of failure, the lengths of time required to restore the components, and descriptions of the failures and the corrective actions. The NRPDS is advantageous to aging research in that it contains an extensive amount of component data from multiple utilities that are not available from other sources. However, actual demand data are missing and some information needed for aging, such as the effect of preventative maintenance, and the tracking of individual components throughout their service lives, generally cannot be obtained from the NRPDS data. At present, the NRPDS is not adequate in itself for plant-specific aging evaluations (Benaroya, 1988).

As a first step in the screening of SSCs for aging analysis, a generic database such as the NPRDS can be used to conduct an aging-survey analysis, such as that described by Meale, et al. (1988). The purpose of such an analysis would be to identify systems and components most affected by aging. NPRDS failure records are classified by utility personnel into nine categories referred to as "cause categories." Of the nine failure categories, the only one corresponding to the generic category of aging is "wear out." In the aging-survey analysis, the fraction of failures of a given component as a result of aging can be found, and those components and systems that are affected significantly by aging can be identified.

As a second step, a reported-cause-of-failure analysis can be conducted. In such an analysis, an examination of the failure records for those components that were identified to be significantly affected by aging can be conducted to identify the dominant reported aging mechanisms and the components most affected. The absolute magnitude of the aging affects are not required, as this will be assessed in the second phase of the aging-risk assessment.

When using generic data bases such as NPRDS in aging-risk analysis, one should be aware that these databases do not often contain information detailed and complete enough for making general conclusions. Events that are not of the direct interest to the reporters are often overlooked or, at most, roughly documented. The contents of the reports are often not detailed enough to allow distinction of an aging-related root cause from other causes. 
The Sequence Coding and Search System Review (SCSS) database contains LERs reported by utilities since 1980. This system provides a structured format for detailed coding of component, system, and unit effects, as well as personnel errors. Each individual event reported in the LER, e.g., component failure, system failure, personnel action, etc., is coded as a separate step in the overall sequence, using information from the entire LER. Specific information, such as cause, system involved, component involved, etc., for each step is encoded. SCSS provides the ability to do general or broad searches at the LER level and more specific searches at the step or sequence level. However, many equipment failures are not reported in LERs because items such as single equipment failures are not reportable under the LER rule (Benaroya, 1988).

Plant-specific data generally contain more detailed information on the operational and failure history of specific systems and components. However, the major limitation of using plant-specific data in aging-risk analysis is that the number of incidents is often too small to reach meaningful statistical conclusion. This is especially true in the case of plants that have only been operated for a few years. One way to improve this situation is to emphasize the root-cause analysis in the existing data base (such as LER or NPRDS). In particular, guidelines should be provided to the plant personnel regarding the reporting of aging-related equipment failures.

Very few plant-specific aging data-analyses have been done in the past. One of these is a Bayesian approach conducted by PLG to evaluate aging trends in equipment failure rate, maintenance rate, and maintenance duration (Bier et al., 1990a, 1990b, 1990c, and 1991). Applying the PLG methodology to plant-specific data from maintenance records of two systems of a BWR plant with $17 \mathrm{yr}$ of operating experience shows some interesting results. Although there is a slight tendency toward increasing failure rates for some components, there is also a slight tendency toward decreasing failure rates for some others. The results of the PLG exercise rule out the likelihood of a rapid change in failure rate because of aging at the specific plant under investigation. However, the rather ambiguous messages regarding the trend of component failure rates indicates that there is a need for further research on this subject.

\subsubsection{PRA and IPE Results}

The results of plant-specific PRAs and IPEs can be useful for identifying components significantly affected by aging that are important to plant safety. A recent study by Vesely (1992) on the prioritization of aging contributors and the evaluation of maintenance effectiveness is an example of such a use. Because most PRAs and IPEs do not include passive components in their plant models, these components would not be identified as risk-significant based on these data sources alone, even though some of them may contribute significantly to aging risk. Consequently, it is important to keep in mind that the decisions made in the screening analysis should be based on sources broader than the PRA and IPE results.

To prioritize passive components according to their risk contributions, it may be necessary to add all the passive components to all the fault trees for all the mechanical systems in a PRA. However, this would be a very large task, and a more approximate approach may be required. Phillips et al. (1992) suggest adding all the passive components to a single system important to the mitigation of core damage and make analogies to the PRA in general.

\subsection{Data Needs for Modeling Aging Mechanisms}

Generally speaking, procedures for reliability data collection have improved significantly over recent years. The recent emphasis that failure times (and modes) should be recorded (rather than just failure counts) has made statistical inference for reliability easier and potentially more powerful. Most data collection in the nuclear industry includes close monitoring of failure times, although some reliability studies still depend on attribute data for important inferences about system and component 
lifetime distributions. The recent use of expert opinion helps fill gaps in reliability studies for which data is lacking or nonexistent. Although such supplemental data are often subjective, it provides a strong basis for prior information in statistical analyses that use Bayesian methods.

The field of statistical reliability literature, and specifically the study of component aging, is vacant of sample size determination formulations. Often, the minimal sample requirements are decided by available funding and resources or approximated using classical formulas that were derived for simpler statistical problems. Derivations for sample size needs in the more complicated life testing problems are often unworkable or unique to the problem at hand. As an alternative, sequential testing and estimation procedures might be substituted [see Mann, Schafer, and Singpurwalla (1974) in sampling situations where block testing is not prerequisite].

Physically based probabilistic models for estimating aging effects on component failure rates often require a large amount of data related to material properties and operating conditions. In the review of degradation mechanisms and associated models performed in Sec. 2, a wide range of data sources was identified.

As indicated previously, a screening procedure would be used to determine components that are critical to the reliability of the entire system, as well as the salient aging and deterioration mechanisms. However, the screening procedure is only a first step, and further efforts are needed to evaluate the status and quality of the data base in each of the identified aging areas. In this section, we assess the status and adequacy of the data base related to the identified aging phenomena.

\subsubsection{Fatigue}

The two basic approaches to fatigue analysis that have been described in Sec. 2 are the cyclesto-failure type methods, which use an S-N curve and Miner's rule or something similar, and the probabilistic mechanics methods, the core of which is some sort of crack propagation law, such as the Paris-Erdogan equation.

With respect to the data needs for a cycles-to-failure type analysis of fatigue lifetime, an extensive database is available for fatigue of steels under a wide range of loading and environmental conditions. These data usually appear in the form of a so-called S-N curve, which relates the number of cycles of failure to the applied load. However, these data are usually deterministic in nature; therefore, to perform a fatigue reliability analysis using a Miner's rule type formulation, as described in Sec. 2, the statistical distribution of the number of cycles to failure for a given applied load, over the range of loads of interest, is required. This type of data does exist for most steels of interest, although they are more limited than that for deterministic S-N curves.

Another type of data that is needed for a probabilistic fatigue lifetime analysis using Miner's rule is that related to the distribution of applied loads. This type of data often can be obtained by examining operating experience and is discussed in more detail later.

The fracture mechanics approach to fatigue analysis is more detailed and is based on the use of a data base for crack propagation that is conveniently incorporated in a Paris-Erdogan-type of crack extension law. Detailed stress analysis is required to predict the propagation rate of fatigue cracks. Probability fracture mechanics adds one more layer of complexity to this modeling effort, where the statistics of the data are included. One such approach is the PRAISE computer code.

It is recommended that such analysis be performed only for certain specific critical components that are at high risk for failure (e.g., the nozzles in the inlet/outlet of the pressure vessel). In these critical components, statistical distribution functions must be determined for initial crack-size 
distributions and for loading conditions. A time-dependent failure rate can be formed from these data and included in an overall PRA analysis.

With respect to data needs for probabilistic fracture mechanics analyses, crack growth rates must be predicted on the basis of a crack growth law, such as the Paris-Erdogan equation, given where $\Delta \mathrm{K}$ is the stress intensity factor range and $\mathrm{C}$ and $\mathrm{m}$ are experimentally determined constants appropriate to the material. In probabilistic fracture mechanics analyses, such as those described in Harris and Lim (1982) and Phillips et al. (1992), probability distributions of the above constants are necessary to obtain a failure probability via Monte Carlo simulation. Numerous sources of data exist for this, such as that described in Bamford (1979), French data (FCFMS, 1979), James and Jones (1985), Shaninian (1975), Van Der Sluys (1982), and General Electric data (Hale, 1978). Special care should be taken when selecting crack growth data, in that crack growth rates can be affected by environmental effects such as corrosion. With that in mind, the environmental conditions under which a component is operating should be assessed to determine whether the available crack propagation data are applicable; if it is not, further research may be necessary to provide the appropriate data.

Data in the ultra-low growth rate regime below $10^{-6} \mathrm{~mm} / \mathrm{cycle}$ (approaching the so-called fatigue threshold) are particularly important as fatigue lifetimes can be dominated by crack extension in this region (Shah, 1987). The need for ultra-low growth rate data is amplified further by estimates of reactor component service loads. For example, service loads at the Zion-1 PWR plant have been estimated to include over $2 \times 10^{10}$ vibrational stress cycles, which over the 40 -yr life of a component could lead to extensive crack growth at the seemingly insignificant near-threshold growth rates (Yahr, et al., 1984).

A second type of data necessary for PFM analyses is that pertaining to the initial distribution of cracks and the nondetection probability of such cracks before the component is put into service. Marshall (1982) presents data from cracks found in nuclear and nonnuclear pressure vessels in the US and UK. Other sources include Becher and Hansen (1974). With respect to the probability of detecting a flaw with ultrasonics or any other nondestructive testing method, the sensitivity and reliability of NDE methods (in particular ultrasonic examinations) have been the subject of research in the Programme of Inspection for Steel Components (PSIC, 1986) and by Taylor, et al. (1983). Other data sources include Harris (1977), Rummel et al. (1977), Tang (1973), and Johnston et al. (1978).

Ideally, plant- and component-specific data should be used to determine the distribution of initial crack sizes and the probability of crack detection because the presence of cracks may be component specific and the probability of detecting cracks is dependent on the type of inspection technique used. Plant maintenance records and in-service inspection reports may be a good source of such information.

The third type of data needed for a probabilistic analysis of fatigue lifetime is the distribution of loads, both in time and magnitude, that a given component will experience. These loads can consist of both operational loads (vibration, flow stratification, etc.) and transient loads (heatup, cooldown, reactor overpower, check valve leaks, etc.). This can be obtained to some extent from plant specific operating experience.

\subsubsection{Irradiation Embrittlement}

This aging mechanism is related mainly to failure of the pressure vessel. As we discussed in Sec. 2, reliability studies are based on probabilistic fracture mechanics, taking into account the reduction of fracture toughness resulting from the DBTT shift with the neutron fluence. The data base for the effects of irradiation on the DBTT is adequate, and efforts should be directed toward statistical descriptions of this data base. 
It is recommended here that a probabilistic methodology be implemented for computations of pressure-vessel failure rates. Because the pressure vessel is one of the high-risk components in the plant, the effort in this area would be well justified. The results of this approach would be a timedependent failure rate that will accurately describe aging of the pressure vessel. It is quite clear from our previous analysis that, as time passes, the DBTT will continue shifting upward and the USE will continue shifting downward in such a way as to decrease critical values of the stress-intensity factors. In simpler terms, the equivalent "strength" of the pressure vessel will decrease continually with age. Therefore, computed failure rates based on a probabilistic model would be increasing functions of time and could be incorporated easily in an overall system-level PRA model.

Embrittlement is normally monitored by testing samples of base, HAZ, and weld materials in the form of CVN and tension specimens that have been irradiated in surveillance capsules located near or adjacent to the vessel wall. This represents an effort to come as close as possible to simulating actual conditions experienced by the vessel itself. Appendix $H$ of 10 CFR 50 requires that the samples be selected from the most limiting material. There are usually several surveillance capsules that are tested at specified intervals over the life of a vessel. Neutron flux in the surveillance capsules is greater than that in the actual vessel by a lead factor that is intended to provide an early warning of unacceptable levels of property degradation. The CVN specimens provide two measures of embrittlement: changes in the USE and shifts in the transition temperature at the 41-J energy level. Data from reactor vessel surveillance programs have been compiled in an EPRI report (Oldfield, 1986) that contains the measured results from 57 reactors and 116 capsules. Also, the Power Reactor Embrittlement Data Base compiled at Oak Ridge National Laboratory (Stallman and Kam, 1991) lists the test results of 117 base materials (plates and forgings, 85 welds, and 88 heat-affected-zone materials that were irradiated in 241 capsules of 82 reactors. The data from the CVN tests, along with information on material chemistry and accumulated fluence, have been included in a data base for development of improved radiation damage prediction methods.

To estimate the probability of vessel failure under a postulated PTS transient, it is necessary to relate the shifts in the DBTT and USE to fracture mechanics material properties. At lower temperatures (in the brittle regime), the linear-elastic crack initiation toughness, $\mathrm{K}_{\mathrm{Ic}}$, and crack arrest toughness, $\mathrm{K}_{\mathrm{Ia}}$, can be used. However, elastic-plastic parameters for characterizing the toughness of steel are needed at higher temperatures. Stable crack growth resistance curves ( $J-R$ curves) often are used for characterizing the upper-shelf toughness, $J_{C}$, and a considerable effort has been made to evaluate the effect of neutron irradiation on them.

Lower bound curves for the relationship of the fracture toughness to temperature (for both $\mathrm{K}_{\mathrm{Ic}}$ and $\mathrm{K}_{\mathrm{Ia}}$ ) are given in App. A of Sec. XI of the ASME Boiler and Pressure Vessel Code. The effects of irradiation on the crack initiation and arrest fracture toughness can be estimated by applying the shift in the RTNDT to shift the ASME lower bound curves for $\mathrm{K}_{I_{c}}$ and $\mathrm{K}_{\mathrm{Ia}}$ by moving the curves by the same shift amount but leaving the shapes unaltered. However, because the ASME lower bound curves are very conservative, it may be better to use actual plant-specific surveillance data for calculating vessel reliability to obtain best-estimate $\mathrm{K}_{\mathrm{IC}}$ and $\mathrm{K}_{\mathrm{Ia}}$ curves and associated uncertainty bounds.

For most plants, it appears that the surveillance program provides an adequate supply of raw data to characterize the fracture mechanics parameters for a plant-specific vessel reliability study. However, for a probabilistic treatment of irradiation embrittlement, it may be necessary to convert the fracture-toughness data base into corresponding statistical data for stress intensity factors.

One area of concern is related to the characterization of an initial flaw distribution. Flaw distributions that are used in the PFM analysis portion of a PTS risk assessment can affect the overall results by several orders of magnitude. They represent uncertainties dealing with the presence, detection, sizing, and location of flaws in critical reactor vessel materials. The current flaw distributions, such as those used in the OCTAVIA code (Lynn, 1977), are not based soundly on relevant 
inspection data, and they are complicated by the aforementioned uncertainties. Therefore, one must be careful that a technically appropriate flaw distribution is used when dealing with an integrity assessment of vessels with low upper shelf toughness behavior (Bamford, 1988).

Another area of concern is the extrapolation of surveillance results for neutron flux and fluence from the capsule locations to the vessel wall. Uncertainties in neutron exposure can be reduced by taking plant-specific measurements in surveillance capsules and ex-vessel reactor cavities to provide normalization points for interpolation and extrapolation into the vessel wall, or by performing plantspecific transport calculations (Lippincott and McElroy, 1983; Norris, 1983).

\subsubsection{Stress-Corrosion Cracking and Erosion/Corrosion}

These two areas of damage mechanisms are much less quantified and analyzed than fatigue and embrittlement. Data on SCC and erosion/corrosion are mainly of fundamental, rather than engineering, value. We recommend that existing phenomenological models be correlated to a wider data base. The models and their associated data bases are not of the probabilistic type and hence cannot be used directly to predict failure rates. The data base should be expanded so that it can describe mean values as well as uncertainties. Analyses similar to what has been presented for fatigue and embrittlement can be performed to predict time-dependent failure rates for these mechanisms.

For modeling efforts for erosion-corrosion, it can be concluded that accurate predictions of erosion-corrosion rates on the basis of laboratory data are still very limited. Existing models are capable of metal-loss predictions within the range of data on which they are based. However, it should be noted that small variations in environmental conditions may lead to unsuspectedly high rates of erosion-corrosion under certain circumstances. These variables, plus significant variations in the rate of mass transfer at specific locations, may be the source of very large differences in erosioncorrosion rates from plant to plant, from component to component, and, in particular, for different locations in the same component even though no changes in the overall hydrodynamic conditions are suspected (Cragnolino, 1987). 


\section{REFERENCES}

Apostolakis, G. and A. Mosleh, "Expert Opinion and Statistical Evidence: An Application to Reactor Core Melt Frequency," Nuclear Science \& Engineering 70, 135-149 (1979).

Apostolakis, G. and P. Moieni, "The Foundations of Models of Dependence in Probabilistic Safety Assessment," Reliability Engineering 18, 177-195 (1987).

Apostolakis, G. and S. Kaplan, "Pitfalls in Risk Calculations," Reliability Engineering 2, 135-145 (1981).

Apostolakis, G. and T. L. Chu, "The Unavailability of Systems Under Periodic Test and Maintenance," Nuclear Technology 50, 5-15 (1980).

Apostolakis, G., "Probability and Risk Assessment: The Subjectivistic Viewpoint and Some Suggestions," Nuclear Safety 19, 305-315 (1978).

Apostolakis, G., S. Kaplan, B. J. Garrick, and R. J. Duphily, "Data Specialization for Plant-Specific Risk Studies," Nuclear Engineering and Design 56, 321-329 (1980).

Apostolakis, G., "The Concept of Probability in Safety Assessments of Technological Systems," Science 250, 1359-1364 (1990).

Apostolakis, G., V. M. Bier, and A. Mosleh, "A Critique of Recent Models for Human Error Rate Assessment," Reliability Engineering and System Safety 22, 201-217 (1988).

ASME, ASME B\&PV Code Section III, Div. 1, Appendix I: Fatigue Table I-9-1, p. 154, 1983a.

ASME, High Cycle Fatigue Design Art. xvii-3000 ASME B\&PV Code Sect III, Div. 1, Appendices 1983, pp. 424-430, $1983 \mathrm{~b}$.

Atwood, C. L., "Parametric Estimation of Time-Dependent Failure Rates for Probabilistic Risk Assessment," Reliability Engineering and System Safety, 37, 181-194 (1992).

Bamford, W. H., et al., "Effects of Low Upper Shelf Toughness on Reactor Vessel Integrity during Pressurized Thermal Shock," in Life Extension and Assessment: Nuclear and Fossil Power-Plant Components, PVP-138, ASME, 1988.

Bamford, W. H., "Fatigue Crack Growth of Stainless Steel Piping in a Pressurized Water Reactor Environment," Journal of Pressure Vessel Technology 101, 73-79 (1979).

Barlow, R. E., and F. Proschan, Statistical Theory of Reliability Theory, Holt, Rinehart and Winston, New York, 1981.

Becher, P. E. and A. Pedersen, "Application of Statistical Linear Elastic Fracture Mechanics to Pressure Vessel Reliability Analyssis," Nuclear Engineering and Design 27, 413-425 (1974).

Becher, P. E. and Hansen, B., Statistical Evaluation of Defects in Welds and Design Implications, Danish Welding Institute, RISO report SRE-11-74 (May 1974).

Benaroya, V., "Aging and NRC Data Sources," in Proceedings of the International Nuclear Power Plant Aging Symposium, Bethesda, MD, August 30-31, 1988. 
Bier, V. M., D. C. Bely, and D. H. Johnson, "Methods of Bayesian Aging Analysis," in Probabilistic Safety Assessment and Management, G. Apostolakis, Ed., Elsevier, New York, pp. 1269-1274, 1991.

Bier, V. M., D. C. Bley, D. H. Johnson, S. Kaplan, and J. E. Williamson, Development and Demonstration of Methods for Nuclear Power Plant Aging Risk Analysis: DORIAN Users Manual, PLG, PLG-0759, Newport Beach, CA, 1990a.

Bier, V. M., D. C. Bley, P. Y. C. Chen, and D. H. Johnson, Development and Demonstration of Methods for Nuclear Power Plant Aging Risk Analysis: Plant-Specific Datn Collection and Interpretation, PLG, PLG-0717, Newport Beach, CA, 1990c.

Bier, V. M., D. C. Bley, Y.-M. Hou, D. H. Johnson, S. Kaplan, and J. E. Williamson, Developinent and Demonstration of Methods for Nuclear Power Plant Aging Risk Analysis: Bayesian Data Analysis, PLG, PLG-0743, Newport Beach, CA, 1990b.

Billinton, R., and R. N. Allan, "Reliability Evaluation of Engineering Systems: Concepts and Techniques," (1983).

Bley, D. C., D. H. Johnson, A. A. Dykes, and V. M. Bier, "Bayesian Aging Analysis: Methods and Applications," from International Conference on Probabilistic Safety Assessment and Management, Beverly Hills, CA, February 1991.

Bogdanoff, J. L. and F. Kozin, Probabilistic Models of Cumulative Damage, J. Wiley and Sons, New York (1985).

Bompas-Smith, J. H., Mechanical Survivability, McGraw-Hill, 1975.

Breeding, R. J., J. C. Helton, E. D. Gorham, and F. T. Harper, "Summary Description of the Methods Used in the Probabilistic Risk Assessments for NUREG-1150," Nuclear Engineering and Design 135, 1-29 (1992).

Breipohl, A. M., "Kalman Filtering and Its Application to Reliability," IEEE Transactions on Reliability 18, No. 3, 127-130 (1969).

Budnitz, R. J., letter to Dr. I. Levy, Battelle Pacific Northwest Laboratory, "Chairman's Letter, Panel to Prioritize NPP Components for Aging Research," 1987.

Carlson, D. D., et al., "Interim Reliability Evaluation Program Procedures Guide," U.S. Nuclear Regulatory Commission report NUREG/CR-2728 (1983).

Chanin, D. I., et al., "MELCOR Accident Consequence Code System (MACCS)," U.S. Nuclear Regulatory Commission report NUREG/CR-4691 (1990).

Chen, D., "A New Approach to the Estimation of Fatigue Reliability at a Single Stress Level," Reliability Engineering and System Safety 33, 101-113, 1991a.

Chen, D., "New Approaches to the Estimation of Cumulative Fatigue Reliability," Reliability Engineering and System Safety 33, 231-247 (1991b).

Cheverton, R. D. and D. G. Ball, "OCA-P, A Deterministic and Probabilistic Fracture-Mechanics Code for Application to Pressure Vessels," U.S. Nuclear Regulatory Commission report NUREG/CR-3618 (1984). 
Chexal, B. and J. Horowitz, "Flow Assisted Corrosion in Carbon Steel Piping-Parameters and Influences," in Symposium on Environmental Degradation of Materials in Nuclear Power Plant Systems-Water Reactors, 1989.

Chhibber, S., and G. Apostolakis, "Some Approximations Useful to the Use of Dependent Information Sources," Reliability Engineering and System Safety, to be published in 1993.

Chhibber, S., G. Apostolakis, and D. Okrent, "On the Use of Expert Judgments to Estimate the Pressure Increment in the Sequoyah Containment at Vessel Breach," Reliability Engineering and System Safety $38,27-45,1992 a$.

Chhibber, S., G. Apostolakis, and D. Okrent, "A Taxonomy of the Use of Expert Judgments in Safety Studies," Reliability Engineering and System Safety 38, 27-45, $1992 \mathrm{~b}$.

Chopra, O. K., "Thermal Aging of Cast Stainless Steels: Mechanisms and Predictions," in Fatigue, Degradation, and Fracture-1990, PVP-Vol. 195, MPC-Vol. 30, ASME, New York, pp.193-214, 1990.

Congleton, J. and I. H. Craig, "Corrosion Fatigue," in Corrosion Processes, R. N. Parkins, Ed., Applied Science Publishers, 1982.

Cooke, R. M., Expert in Uncertainty: Expert Opinion and Subjective Probability in Science, Oxford University Press, New York, 1991.

Cornell, C. A., "Engineering Seismic Risk Analysis," Bulletin of the Seismological Society of America 58, 1583-1606 (1968).

Courtnall, M. and F. B. Pickering, "The Effect of Allying on $485^{\circ} \mathrm{C}$ Embrittlement," Metal Science, 8 , p. 273 (1976).

Cox, D. R. and P. A. W. Lewis, The Statistical Analysis of Series of Events, Chapman and Hall, London, 1966.

Cragnolino, C., "A Review of Erosion-Corrosion in Steels in High Temperature Water," in Environmental Degradation of Materials in Nuclear Power Systems - Water Reactors, The Metallurgical Society, 1987.

Crow, L. H., "Confidence Interval Procedures for the Weibull Process with Applications to Reliability Growth," Technometrics 24, No. 1, 67-72 (1982).

Ditlevsen, O. and K. Sobczyk, "Random Fatigue Crack Growth with Retardation," Engineering Fracture Mechanics 24, 861-878 (1986).

Eason, E. D. and E. E. Nelson, "Multivariable Modeling of Pressure Vessel and Piping J-R Data," U. S. Nuclear Regulatory Commission report NUREG/CR-5729 (1991).

Efron, B., "Efficiency of Cox's Likelihood Function for Censored Data," Journal of the American Statistical Association 72, 557-565 (1977).

Embrey, D. E., " SLIM-MAUD: A Computer-Based Technique for Human Reliability Assessment," Proc. International ANS/ENS Topical Meeting on Probabilistic Safety Methods and Applications, San Francisco, California, 1985. 
Embrey, D. E., P. C. Humphreys, E. A. Rosa, B. Kirwan, and K. Rea, "SLIM-MAUD: An Approach to Assessing Human Error Probabilities Using Structured Expert Judgment," U.S. Nuclear Regulatory Commission report NUREG/CR-3518 (1984).

Englehardt, M., "Weibull Processes," in Encyclopedia of Statistical Sciences, Vol. 9, S. Kotz \& N. L. Johnson, Eds., John Wiley and Sons, New York, 1988.

EPRI, 1987a, "Single-Phase Erosion/Corrosion of Carbon Steel Piping," EPRI report, February 19, 1987.

EPRI, 1987b, EPRI Workshop on Erosion/Corrosion of Carbon Steel Piping, Washington, D. C., April 14$15,1987$.

EPRI, 1987c, "Nondestructive Examination of Ferritic Piping for Erosion/Corrosion," EPRI draft report, April 1987.

EPRI, 1988, "Acceptance Criteria for Structural Evaluation of Erosion-Corrosion Thinning in Carbon Steel Piping," EPRI report NP-5911M, Project 1757-61, July 1988.

FCFMS, "Influence of Various Parameters on the Determination of the Fatigue Crack Arrest Threshold," Fatigue Commission of the French Metallurgical Society, presented at the ASTM meeting, Pittsburgh, PA, 1979.

Fleming, K. N., "A Redundant Model for Common-Mode Failures in Redundant Safety Systems," presented at the 6th Annual Conference on Modeling and Simulation, Pittsburgh, Pennsylvania, April 24-25, 1975.

Fleming, K. N., A. Mosleh, and R. K. Deremer, "A Systematic Procedure for the Incorporation of Common Cause Events into Risk and Reliability Models," Nuclear Engineering and Design 93, 245-273 (1986).

Flinn, R. A. and P. K. Trojan, Engineering Materials and Their Application, Houghton Mifflin Company, Boston, MA, 1990.

Ford, F. P., "Stress Corrosion Cracking," in Corrosion Processes, R. N. Parkins, Ed., Applied Science Publishers, 1982.

Fullwood, R. R. and R. E. Hall, "PRAISEDPD: An Aging Pipe Reliability Analysis PC Code," Reliability Engineering and System Safety, 30, 427-446 (1990).

Georgin, J. P. and J. P. Signoret, "The Maximum Likelihood Estimate from a Confidence Level Points of View-Proposition for an Improved One," Reliability Engineering 2, 259-269 (1981).

Gumbel, E. J., Statistics of Extremes, Columbia University Press, New York, 1958.

Gurney, T. R., "Fatigue Design Rules for Welded Steel Joints," Welding Institute Research Bulletin, Vol. 17 (1976).

Guthrie, G. L., "Charpy Trend Curves Based on 177 PWR Data Points," in LWR Pressure Vessel Surveillance Dosimetry Improvement Program, Quarterly Progress Report, U.S. Nuclear Regulatory Commission report NUREG/CR-3391, Vol. 1 (1983).

Guthrie, G. L., "Charpy Trend Curves Bases on 177 PWR Data Points," in LWR Pressure Vessel Suroeillance Dosimetry Improvement Program, Quarterly Progress Report, U.S. Nuclear Regulatory Commission report U.S. Nuclear Regulatory Commission report NUREG/CR-3391, Vol. 2 (1983). 
Hale, D. A., et al., "Fatigue Crack Growth in Piping and RPV Steels in Simulated BWR Water Environment," GEAP-24098, 1978.

Hannaman, G. W., V. Joksimovich, D. H. Worledge, and A. J. Spurgin, "The Role of Human Reliability Analysis for Enhancing Crew Performance," Proc. International ANS/ENS Topical Meeting on Adoances in Human Factors in Nuclear Power Systems, Knoxville, Tennessee, 1986.

Harris, D. O. and E. Y. Lim, "Applications of a Fracture Mechanics Model of Structural Reliability to the Effects of Seismic Events on Reactor Piping," Progress in Nuclear Energy 10, 125-159 (1982).

Harris, D. O., "An Analysis of the Probability of Pipe Rupture at Various Locations in the Primary Cooling Loop of a Babcock and Wilcox 177 Fuel Assembly Pressurized Water Reactor - Including the Effects of Periodic Inspection," Science Applications Inc. report SAI-050-77-PA, Palo Alto, CA, 1977.

Harris, D. O., E. Y. Lim, D. D. Dedhis, H. H. Woo, and C. K. Chou, "Fracture Mechanics Models Developed for Piping Reliability Assessment in Light Water Reactors," U.S. Nuclear Regulatory Commission report NUREG/CR-2301 (1981).

Harrison, J. D. and S. J. Maddox, Derivation of Design Rules for Pressure Vessels, XIII-941-80, The Welding Institute, 1980.

Hawthorne, J. R., Ed., "The NRL-EPRI Research Program (RP886-2) Evaluation and Prediction of Neutron Embrittlement in Reactor Pressure Vessel Materials, Annual Progress Report for FY 1978," NRL. report 8327, Naval Research Laboratory, Washington, DC, 1979c.

Hawthorne, J. R., "Evaluation of IAEA Coordinated Program Steels and Welds for $288^{\circ} \mathrm{C}$ Radiation Embrittlement Resistance," Presented to IWG-RRPC Coordinated Program Review Meeting, Vienna, Austria, 22-23 October 1981, NRL Memorandum Report 4655, February 1982.

Hawthorne, J. R., H. E. Watson, and F. J. Loss, in Effects of Radiation on Materials, ASTM STP 725, American Society for Testing and Materials, pp. 63-75, 1982.

Hawthorne, J. R., "IARAR Program Investigations," Ninth Annual NRC Water Reactor Safety Review Meeting, Nuclear Regulatory Commission, Gaithersburg, MD, October 1981.

Hawthorne, J. R., J. J. Kozoil, and S. T. Byrne, in Effects of Radiation on Structural Materials, ASTM STP 683, American Society for Testing and Materials, pp.235-251, 1979a.

Hawthorne, J. R., Nuclear Technology 59, No. 3, pp. 441-455.

Hawthorne, J. R., "Survey of Postirradiation Heat Treatment as to Means to Mitigate Radiation Embrittlement of Reactor Vessel Steels," U.S. Nuclear Regulatory Commission report NUREG/CR 0486 (February 1979).

Henzel, N., W. Kastener, and B. Stellwag, "Erosion/Corrosion in Power Plants Under Single- and TwoPhase Flow Conditions," presented at American Power Conference, 50th Annual Meeting, Chicago, IL, April 1988.

Hiser, A. L., "Tensile and J-R Curve Characterization of Thermally Aged Austenitic Cast Stainless Steel," in Properties of Stainless Steels in Eleoated Temperature Seroice, MPC-Vol. 26, PVP-Vol. 132, ASME, New York, 1987.

Hoel, P. G., Introduction to Mathematical Statistics, 4th ed., John Wiley \& Sons, Inc., New York, 1971. 
Hollander, M., and F. Proschan, "Testing Whether New is Better than Used," Annals of Mathematical Statistics 43, 1136-1146 (1972).

Hoopingarner, H. R., J. W. Vause, D. A. Dingee, and J. F. Nesbitt, "Aging of Nuclear Station Diesel Generators: Evaluation of Operating and Expert Experience," U.S. Nuclear Regulatory Commission report NUREG/CR-4590 (1987).

Hubble, W. H. and C. F. Miller, "Data Summaries of Licensee Event Reports of Valves at U.S. Commercial Nuclear Power Plants, January 1, 1976, to December 31, 1978," U.S. Nuclear Regulatory Commission report NUREG/CR-1363 (1980).

IAEA, "Coordinated Research Programme on Analysis of the Behavior of Advanced Reactor Pressure Vessel Steels Under Neutron Irradiation," IWG RRPC-78/81, International Atomic Energy Agency, Vienna, Austria, 17-18 October 1977.

"IEEE Guide to the Collection and Presentation of Electrical, Electronic, and Sensing Component Reliability Data for Nuclear Power Generation Stations," IEEE STD-500, 1977.

INPO, "Pipe Failures in High Energy Systems due to Erosion/Corrosion," INPO-SOER 87-3, March 20, 1987.

James, L. A. and Jones, D. P., "Fatigue Crack Growth Correlations for Austenitic Stainless Steels in Air," in Predictive Capabilities in Environmentally Assisted Cracking, Proceedings of the Winter Annual Meeting of the American Society of Mechanical Engineers, Miami Beach, Florida, November 17-22, PVP Vol. 99, 1985.

Jarrell, D. B., A. B. Johnson, Jr., P. W. Zimmerman, and M. L. Gore, "Nuclear Plant Service Water System Aging Degradation Assessment, Phase I," U.S. Nuclear Regulatory Commission report NUREG/CR-5379, Vol. 1 (1989).

Jaske, C. E. and V. N. Shah, "Life Assessment Procedures for Major LWR Components," U.S. Nuclear Regulatory Commission report NUREG/CR-5314 (1990).

Johnston et al., "What are the Chances of Failure? Part 2, NDT, Pressure Vessel Applications and Fatigue," Welding Institute Research Bulletin 19, 78-82 (1978).

Jouris, G. M. and F. J. Witt, "An Application of Probabilistic Fracture Mechanics to Reactor Pressure Vessels Including Multiple Initiation and Arrest Events," SMiRT 7, Vol. M, Chicago, IL, 1-8, 1983.

Kaplan, E. L. and Meier, P., "Nonparametric Estimation from Incomplete Observations," Journal of the American Statistical Association 53, 457-481 (1958).

Kaplan, S. and B. J. Garrick, "On the Quantitative Definition of Risk," Risk Analysis 1, 11-37 (1981).

Kaplan, S., G. Apostolakis, B. J. Garrick, D. C. Bley, and K. Woodard, "Methodology for Probabilistic Risk Assessment of Nuclear Power Plants," Pickard, Lowe and Garrick, Inc. report PLG-0209, Irvine, California, 1981.

Kaplan, S., "On a 'Two-Stage' Bayesian Procedure for Determining Failure Rates from Experiential Data," IEEE Trans. Power Apparatus and Systems, PAS-102, 195 (1983).

Kapur, K. C. and L. R. Lamberson, Reliability in Engineering Design, John Wiley and Sons, 1977. 
Keeney, R. L. and von Winterfeldt, D., "Eliciting Probabilities from Experts in Complex Technical Problems," IEEE Transactions on Engineering Management 38, 191-201 (1991).

Keeney, R. L. and von Winterfeldt, D., "On the Uses of Expert Judgment on Complex Technical Problems," IEEE Transactions on Engineering Management 36, 83-86 (1989).

Kennedy, R. P., et al, "Probabilistic Seismicity Safety Study of an Existing Nuclear Power Plant," Nuclear Engineering and Design 59, 315-338 (1980).

Klefsjo, B., "Some Tests Against Aging Based on the Total Time on Test Transform," University of Umea Statistical Research (revision of Report No. 1979-4), 1980.

Klinger, D. J., "Failure Time and Rate Constant of Degradation: An Argument for the Inverse Relationship," to appear in Microelectronics and Reliability (1992).

Lawless, J. F., Statistical Models and Methods for Lifetime Data, John Wiley \& Sons, New York, 1988.

Lee, L. and Lee, S. K., "Some Results on Inference for the Weibull Process," Technometrics 1, Vol. 20, 4146 (1978).

Lehman, E. L., Nonparametrics : Statistical Methods Based on Ranks, Holden-Day, San Francisco, CA, 1975.

Levy, I. S., J. Wreathall, G. Demoss, A. Wolford, E. P. Collins, and D. B. Jarrell, "Prioritization of TIRGALEX-Recommended Components for Further Aging Research," U.S. Nuclear Regulatory Commission report NUREG/CR 5248 (1988).

Lewis, W. H. et al., Reliability of Non-Destructive Inspection, San Antonio Air Logistics Center report SA-SLC/MME 76-6-38-1, 1978.

Lin, Y. K., W. F. Wu and J. N. Yang, "Stochastic Modeling of Fatigue Crack Propagation," in Probabilistic Methods of Solids and Structures, Springer-Verlag, Berlin, 103-110, 1984.

Lippincott, E. P. and W. N. McElroy, "Determination of Neutron Damage Parameters for Irradiation Embrittlement," in Irradiation Embrittlement and Suroeillance of Nuclear Reactor Pressure Vessels: an International Study, ASTM STP 819, 1983.

Lois, E., "NUREG/CR-5510 Methodology for Aging Risk," memorandum to G. Weidenhammer, US NRC, July 21, 1992.

Loss, F. J., B. H. Menke, R. A. Gray, J. R. Hawthorne, and H. E. Watson, in Effects of Radiation on Materials, ASTM STP 725, American Society for Testing and Materials, pp. 76-91, 1982.

LuValle, M., "Experiment Design to Explore Multi-step, Multi-stress Failure Modes" in Advances in Reliability, A. P. Basu, Ed., Elsvier Science Publishers, New York (1992).

Lynn, E. K., "The OCTAVIA code for Predicting Vessel Performance and Failure Probabilities," Fifth Water Reactor Safety Meeting, Gaithersburg. MD, 1977.

Mager, T. R., Ed., Feasibility and Methodology for Thermal Annealing of an Embrittled Reactor Vessel, EPRI RP1021-1, Semiannual Technical Progress Report No. 2, Electric Power Research Institute, Palo Alto, California, July 1978. 
Manjoine, M. J. and B. C. Gowda, "Fatigue Design and Testing for Pressure Vessels and Piping Applications," Pressure Vessel Technology, Vol. 2, Materials and Fabrication, p. 735, L. Gengdian and R. W. Nichols, Eds., Pergamon Press, New York.

Manjoine, M.J. and R.L. Johnson, Development of Fatigue Design Curves for Ferritic Steels up to $700^{\circ} \mathrm{F}$ $\left(371^{\circ} \mathrm{C}\right)$, ASME-PVP Vol. 109 Book No. 600360, 1986.

Mann, N. R., R. E. Schafer, and N. D. Singpurwalla, Methods for Statistical Analysis of Reliability and Life Data, John Wiley and Sons, Inc., New York, 1974.

Marshall, W., An Assessment of the Integrity of PWR Pressure Vessels, United Kingdom Atomic Energy Authority H. M. Stationery Office London, 1982.

Marshall, W., An Assessment of the Integrity of PWR Pressure Vessels, United Kingdom Atomic Energy Authority, H. M. Stationary Office, London, 1976.

Martz, H. F., and Waller, R. A., Bayesian Reliability Analysis, John Wiley and Sons, Inc., New York, 1982.

Martz, H. F., Campbell, K., and Davis, H. T., "Estimating and Forecasting Failure Rate Processes by Means of the Kalman Filter," from The Theory and Application of Reliability, editors C. P. Tsokos and I. N. Shimi, Academic Press, Inc., New York, 1977.

Meale, M. M. and D. G. Sutterwhite, "An Aging Failure Survey of Light Water Reactor Safety Systems and Components," U.S. Nuclear Regulatory Commission report NUREG/CR-4747 (1988).

Metal Properties Council Subcommittee on Nuclear Materials, Journal of Testing and Evaluation 11, No. 4, pp. 237-260 (July 1983).

Meyer, M. A. and Booker, J. M., Eliciting and Analyzing Expert Judgment, Academic Press (1991).

Meyzaud, Y. and R. Cozar, "Design of Aging-Resistant Martensitic Steels for Pressurized Water Reactors," Proceedings of Topical Conference of Ferritic Alloys for Use in Nuclear Energy Technologies, J. W. Davis and D. J. Michel, Eds., AIME Publication (1984).

Miller, R. G., "Survival Analysis," Wiley Series in Probability and Mathematics, John Wiley and Sons, Inc., New York, 1981.

Mosleh, A. and N. O. Siu, "A Multi-Parameter, Event-Based Common-Cause Failure Model," presented at the 9th International Conference on Structural Mechanics in Reactor Technology, Lausanne, Switzerland, August 1987.

Mosleh, A. (Ed.), Reliability Engineering System Safety, A Special Issue on Dependent Failure Analysis 24, 243-248 (1990).

Mosleh, A., K. N. Fleming, G. W. Parry, H. M. Paula, D. H. Worledge, and D. M. Rasmuson, "Procedures for Treating Common Cause Failures in Safety and Reliability Studies," U.S. Nuclear Regulatory Commission report NUREG/CR-4780 (1988).

Murata, K. K., et al., "User's Manual for Contain 1.1: A Computer Code for Severe Nuclear Accident Containment Analysis," U.S. Nuclear Regulatory Commission report NUREG/CR-5026 (1989).

Natesan, K. and Y. Y. Liu, "Erosion-Corrosion of Materials at Elevated Temperatures," Material Science and Engineering A121, 571 (1989). 
Norris, E. B., "A Service Laboratory's View Of the Status and Direction of Reactor Vessel Surveillance," in Irradiation Embrittlement and Suroeillance of Nuclear Reactor Pressure Vessels: an International Study, ASTM STP 819, 1983.

Odette, G. R. and P. M. Lombrozo, Physically Based Regression Correlations of Embrittlement Data from Reactor Pressure Vessel Surveillance Programs, NP-3319, EPRI, 1984.

Odette, G. R. and T. J. Griesbach, "Radiation Embrittlement Trend Curves and the Impact on Nuclear Plant Operating Criteria," in Radiation Embrittlement of Nuclear Reactor Pressure Vessels, Vol. 3, ASTM STP 1101, 1987.

Oldfield W., Nuclear Plant Irradiated Steel Handbook, NP-4749, EPRI, 1986.

Oliver, R. and W. Ritter, Catalogue of S-N Curoes of Welded Joints in Structural Steel, Part 1, Butt Joints, Deutcher Verlag für Schweisstechnik, Dusseldorf, 1979.

Ortiz, K. and A. S. Kiremidjian, "Stochastic Modeling of Fatigue Crack Growth," Engineering Fracture Mechanics 29, 657-676 (1988).

Ortiz, N. R., et al., "Use of Expert Judgment in NUREG-1150," Nuclear Engineering and Design 135 (1992).

Pacific Gas and Electric Company (PG\&E), "Diablo Canyon Power Plant Units 1 and 2 Individual Plant Examination Report," 1992.

Palme, H. S., "Radiation Embrittlement Sensitivity of Reactor Pressure Vessel Steels," BAW-10056A, Babcock and Wilcox, Lynchburg, VA, August 1973.

Parkins, R. N., "Constant Strain Rate Stress Corrosion Testing," in Stress Corrosion Research, H. Arup and R. N. Parkins, Eds., Sijtoff and Noordhoff Publishers, 1979.

Parkins, R. N., "Current Understanding of Stress-Corrosion Cracking," JOM, 12-19 (1992).

Phillips, J. H., T. W. Bolander, M. L. Magleby, V. A. Geidl, and G. H. Weidenhamer, "Investigation of the Risk Significance of Passive Components using PRA Techniques," presented at the ASME Pressure Vessel \& Piping Conference, New Orleans, LA, June 21-25, 1992.

Pickard, Lowe and Garrick, Inc. (PLG) and Stone \& Webster Engineering Corporation, "Beaver Valley Unit 2 Probabilistic Risk Assessment," PLG-0730, Newport Beach, California, 1990.

Prentice, R. L. and J. E. Kalbfleisch, "Hazard Rate Models with Covariates," Biometrics 35, No. 1, 2540 (1979).

Program for the Inspection of Steel Components (PSIC-II), "Evaluation of the PSIC-II Trials Results," PSIC-II Report No. 5, Ispra, Italy, 1986.

Rasmuson, D. M., "A Comparison of the Small and Large Event Tree Approaches used in PRAs," Reliability Engineering and System Safety 37, 79-90 (1992).

Reichenbach, H., The Theory of Probability: An Inquiry into the Logical and Mathematical Foundations of the Calculus of Probability, English translation by E.H. Hutten and M. Reichenbach, University of California Press, Berkeley, 1949. 
Rummel, W. D., et al., "The Detection of Fatigue Cracks by Non-Destructive Test Methods," Materials Evaluation 32, 205-212.

Russell, K. D., et al., "Integrated Reliability and Risk Analysis System (IRRAS), Version 2.5 Reference Manual," U.S. Nuclear Regulatory Commission report NUREG/CR-5300 (1991).

S. M. Stoller Corp., since 1985, Nuclear Power Experience, 1919 14th Street, Suite 500, Boulder, CO 80302.

Salvia, A. A., "Maximum Likelihood Estimation of Linear Failure Rate," IEEE Transactions on Reliability, R-29, 49-50, 1980.

Samanta, P. K., W. E. Vesely, F. Hsu, and M. Subudhi, "Degradation Modeling with Application to Aging and Maintenance Effectiveness," U.S. Nuclear Regulatory Commission report NUREG/CR-5612, 1991.

Sampath, S. and D. Broek, "Estimation of Requirements of Inspection Intervals for Panels Susceptible to Multiple Site Damage," in Structural Integrity of Aging Aircraft, Springer-Verlag, Berlin, 1991.

Schilling, R., and P. Dorre, "Basic Aspects of Stochastic Reliability Analysis for Redundancy Systems," Reliability Engineering and System Safety 34, 345-354 (1991).

Science Application International Incorporated (SAIC), "CAFTA User's Manual, Version 2.0," prepared for Electric Power Research Institute (EPRI), 1988.

Shah, V. N. and P. E. MacDonald, Eds., Residual Life Assessment of Major Light Water Reactor Components - Overoiew, U.S. Nuclear Regulatory Commission report NUREG/CR-4731, Vols. 1 and 2 (1987).

Shah, V. N. and P. E. MacDonald, Residual Life Assessment of Major Light Water Reactor Components-Overoiew, U.S. Nuclear Regulatory Commission report NUREG/CR-4731 (1989).

Shanihan, P., "Creep-Fatigue Crack Propagation in Austenitic Stainless Steel," Journal of Pressure Vessel Technology, 1979.

Shaw, L., M. Shooman, and R. Schatz, "Application of Time Dependent Stress-Strength Interference Models of Non-Electrical and Electrical Systems, Part I," Proceedings of the Annual Reliability and Maintainability Symposium, 462-67, 1980.

Shaw, L., M. Shooman, and R. Schatz, "Application of Time Dependent Stress-Strength Interference Models of Non-Electrical and Electrical Systems, Part II," Proceedings of the Annual Reliability and Maintainability Symposium, 540-47, 1981.

Shen, K., "On the Relationship Between Component Failure Rate and Stress-Strength Distributional Characteristics," Microelectronics and Reliability 28, 801-12 (1988).

Simonen, E. P. and D. W. Engel, "VISA II-A Computer Code for Predicting the Probability of Reactor Pressure Vessel Failure," U.S. Nuclear Regulatory Commission report NUREG/CR-4486 (1986).

Simonen, E. P. and K. I. Johnson, "VISA-II Sensitivity Study of Code Calculations Input and Analytical Model Parameters," U.S. Nuclear Regulatory Commission report NUREG/CR-4614, 1986a.

Simonen, E. P. and K. I. Johnson, "Vessel Integrity Simulation (VISA) Code Sensitivity Study," U.S. Nuclear Regulatory Commission report NUREG/CR-4267 (1986b). 
Sobczyk, K. and B. F. Spencer, Jr., Random Fatigue: From Data to Theory, Academic Press, Boston, 1992.

Sobczyk, K. and J. Trebicky, "Modeling of Random Fatigue by Cumulative Jump Processes," Engineering Fracture Mechanics 34, 477-493 (1989).

Sobczyk, K., "Stochastic Modeling of Fatigue Crack Propagation," in Probabilistic Methods in the Mechanics of Solids and Structures, Springer-Verlag, Berlin, 111-120, 1984.

Stack, D.W., "SETS User's Manual for Accident Sequence Analysis," U.S. Nuclear Regulatory Commission report NUREG/CR-3547 (1984).

Stallmann, F. W. and F. B. K. Kam, "Power Reactor Embrittlement Data Base," U.S. Nuclear Regulatory Commission report NUREG/CR-4816, Version 1 (1986).

Summers, R. M., et al., "MELCOR 1.8.0: A Computer Code for Nuclear Reactor Severe Accident source And Risk Assessment Analyses," U.S. Nuclear Regulatory Commission report NUREG/CR-5531, 1991.

Swain, A. D. and H. E. Guttmann, "Handbook of Human Reliability Analysis with Emphasis on Nuclear Power Applications," U.S. Nuclear Regulatory Commission report NUREG/CR-1278, Washington, DC, 1983.

Tang, WE. H., "Probabilistic Updating of Flaw Information," Journal of Testing and Evaluation 1, 459467 (1973).

Taylor, T. T., et al., Detection of Small-Sized Near-Surface Under-Clad Cracks for Reactor Pressure Vessels, U.S. Nuclear Regulatory Commission report NUREG/CR-2878, 1983.

The Welding Institute, Design Recommendations for Cyclic Loaded Welded Steel Structures, The International Institute of Welding, IW, Document XV-494-81, 1982.

Thomas, H. M., "Pipe and Vessel Failure Probability," Reliability Engineering 2, 83-124 (1981).

U.S. Nuclear Regulatory Commission (USNRC), "Analysis of the LaSalle Unit 2 Nuclear Power Plant: Risk Methods Integration and Evaluation Program (RMIEP)," NUREG/CR-4832, Washington, DC, 1992.

U.S. Nuclear Regulatory Commission (USNRC), "PRA Procedure Guide-A Guide to the Performance of Probabilistic Risk Assessments for Nuclear Power Plants," NUREG/CR-2300, Washington, DC, 1983.

U.S. Nuclear Regulatory Commission (USNRC), "Reactor Safety Study," WASH-1400, NUREG75/014, Washington, DC, 1975.

U.S. Nuclear Regulatory Commission (USNRC), "Severe Accident Risks: An Assessment for Five U.S. Nuclear Power Plants," NUREG-1150, Washington, DC, 1990.

USNRC, "Nuclear Plant Aging Research (NPAR) Program Plan, Status and Accomplishments, Revision 2," U.S. Nuclear Regulatory Commission report NUREG-1144 (1991).

USNRC, "Thinning of Pipe Walls in Nuclear Power Plants," U.S. Nuclear Regulatory Commission Bulletin 87-01, July 9, 1987.

Van Der Sluys, W. A., Corrosion Fatigue Characteristics of Reactor Pressure Vessel Steels, EPRI NP$2775,1982$. 
Varsik, J. D., and S. T. Byrne, in Effects of Radiation on Structural Materials, ASTM STP 683, American Society for Testing and Materials, pp. 252-266, 1979.

Vesely, W. E., "Approaches for Age-Dependent Probabilistic Safety Assessments with Emphasis on Prioritization and Sensitivity Studies," U.S. Nuclear Regulatory Commission report NUREG/CR-5587 (1992).

Vesely, W. E., "Estimating Common Cause Failure Probability in Reliability and Risk Analysis: Marshall-Olkin Specializations," in Nuclear System Reliability Engineering and Risk Assessment, J. B. Fussell and G. R. Burdick, Eds., SIAM, Philadelphia, 314-341, 1977.

Vesely, W. E., F. F. Goldberg, N. H. Roberts and D. F. Haasl, "Fault Tree Handbook," U.S. Nuclear Regulatory Commission report NUREG-0492 (1981).

Vesely, W. E., "More Comprehensive Approaches for Determining Risk Sensitivities to Component Aging," Nuclear Engineering and Design 106, 389-397 (1988).

Vesely, W. E., R. E. Kurth, and S. M. Scalzo, "Evaluation of Core Melt Frequency Effects Due to Component Aging and Maintenance," U.S. Nuclear Regulatory Commission report NUREG/CR-5510 (1990).

Vesely, W. E., "Risk Evaluations of Aging Phenomena: The Linear Aging Reliability Model and Its Extensions," U.S. Nuclear Regulatory Commission report NUREG/CR-4769 (1987).

Virginia Power Company, Surry Unit 2 Reactor Trip and Feedwater Pipe Failure Report, Rev. 0, Virginia Power Company, January 14, 1987.

von Mises, R., Probability, Statistics, and Truth, English translation by J. Neyman, D. Sholl, and E. Rabinowitsch, B. W. Hodge, London, 1939.

Wei, R. P., "The Fracture Mechanic Approach to Stress Corrosion Cracking," in Stress Corrosion Research, H. Arup and R. N. Parkins, Eds., Sijtoff and Noordhoff Publishers, 1979.

Westinghouse Savannah River Company, 1990. Savannah River Site PRA of Reactor Operation, Level I - External Events, WSRC-RP-89-733.

Wheeler, T. A., et al., Analysis of Core Damage Frequency from Internal Events: Expert Judgment Elicitation, NUREG/CR-5116, Vols. 1 and 2, 1989.

Williams, R. O., "Theory of Precipitation Hardening: Isotopically Strained Systems," Act Met. 5, 385 (1957).

Wolford, A. J., Atwood, C. L., and Roesener, W. S., "Aging Data Analysis and Risk AssessmentDevelopment and Demonstration Study," U. S. Nuclear Regulatory Commission report NUREG/CR5378, EGG-2567 (1992).

Worrell, R. B., "SETS Reference Manual," U.S. Nuclear Regulatory Commission report NUREG/CR4213 (1985).

Wreathall, J., "Operator Action Trees (OATs) Method," Proc. Workshop on Human Factors and Nuclear Safety, IEEE, Myrtle Beach, South Carolina, 1981.

Wright, I. G., V. Nagarajan, and J. Stringer, Oxid. Met. 25, 175, 1986. 
Wu, J. S., G. Apostolakis and D. Okrent, "On the Inclusion of Organizational and Managerial Influences in Probabilistic Safety Assessments of Nuclear Power Plants," presented at the Society for Risk Analysis Annual Meeting, San Francisco, California, October 29-November 1, 1989.

Yahr, G. T., R. C. Gwaltney, A. K. Richardson, and W. L. Server, "Case Study of the Propagation of a Small Flaw under PWR Loading Conditions and Comparison with the ASME Code Design Life," U.S. Nuclear Regulatory Commission report NUREG/CR-3982 (1984).

Yang, J. N., "Statistical Estimation of Cracks and Maintenance Cost for Aircraft Structures," Journal of Aircraft 13, 929-937 (1976).

Yanichko, S. E. and J. N. Chirigos, Nuclear Engineering and Design 56, pp.297-307 (1980). 


\begin{tabular}{|c|c|}
\hline $\begin{array}{l}\text { NAC FORM } 335 \\
\text { (2:-89, } \\
\text { NRCM 1102. } \\
\text { 3201, 3202, } \\
\text { BIB. NUCLEAR REGULATORY COMMISSION } \\
\end{array}$ & $\begin{array}{l}\text { 1. REPORT NUMBER (Assignedby NRC,Add Vol, SUPp.. } \\
\text { Rev., and Addendum Numbers, it any.) } \\
\text { NUREG/CR-6157 } \\
\text { LA-12715-MS }\end{array}$ \\
\hline \multirow{3}{*}{$\begin{array}{l}\text { 2. TITLE AND SUBTITLE } \\
\text { SURVEY·AND EVALUATION OF AGING RISK ASSESSMENT } \\
\text { METHODS AND APPLICATIONS }\end{array}$} & DATE REPORT PUBLISHED \\
\hline & $\begin{array}{ccc}\text { MONTH } & 1 & \text { YEAR } \\
\text { November } & & 1994\end{array}$ \\
\hline & $\begin{array}{l}\text { 4. FIN OR GRANT NUMBER } \\
\text { L2178 }\end{array}$ \\
\hline \multirow{2}{*}{$\begin{array}{l}\text { 5. AUTHOR(S) } \\
\text { D. Sanzo, P. Kvam, G. Apostolakis, J. Wu, T. Milici, N. Ghoniem, } \\
\text { S. Guarro* }\end{array}$} & $\begin{array}{l}\text { 6. TYPE OF REPORT } \\
\text { Technical }\end{array}$ \\
\hline & 7. PERIOD COVERED (Indusive Dates) \\
\hline
\end{tabular}
name and malling addrass.)

Los Alamos, National Laboratory

Los Alamos, New Mexico 87545

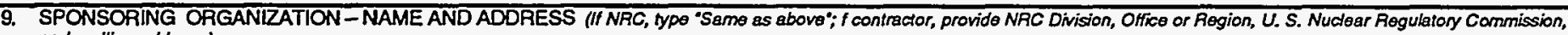
and malling addrass.)

Division of Safety Issue Resolution

Office of Nuclear Regulatory Research

U. S. Nuclear Regulatory Commission

Washington, D. C. 20555

10. SUPPLEMENTARY NOTES

* Advanced System Concepts Associates, 2250 E. Imperial Hwy., Suite 200, El Segundo, CA 90245-3547

\section{ABSTRACT (200 words or less)}

The US Nuclear Regulatory Commission initiated the nuclear power plant aging research program about 6 years ago to gather information about nuclear power plant aging. Since then, this program has collected a significant amount of information, largely qualitative, on plant aging and its potential effects on plant safety. However, this body of knowledge has not yet been integrated into formalisms that can be used effectively and systematically to assess plant risk resulting from aging, although models for assessing the effect of increasing failure rates on core damage frequency have been proposed:

This report surveys the work on the aging of systems, structures, and components (SSCs) of nuclear power plants, as well as associated data bases. We take a critical look at the need to revise probabilistic risk assessments (PRAs) so that they will include the contribution to risk from plant aging, the adequacy of existing methods for evaluating this contribution, and the adequacy of the data that have been used in these evaluation methods. We identify a preliminary framework for integrating the aging of SSCs into the PRA and include the identification of necessary data for such an integration.

12 KEY WORDSIDESCRIPTORS (List words or phrases that will essist ressarchers in locating the report.)

Aging risk assessment methods, system analysis, accident identification

\begin{tabular}{l} 
13. $\begin{array}{l}\text { AVAILABILITY STATEMENT } \\
\text { Unlimited }\end{array}$ \\
\hline $\begin{array}{l}\text { 14. SECURTY CLASSIFICATION } \\
\text { (This Page) } \\
\text { Unclassified } \\
\text { (This Report) } \\
\text { Unclassified }\end{array}$ \\
\hline 15. NUMBER OF PAGES \\
\hline 16. PRICE \\
\hline
\end{tabular}

